\title{
Cochlear implantation in adults with early-onset deafness
}

Citation for published version (APA):

Debruyne, J. A. (2019). Cochlear implantation in adults with early-onset deafness. [Doctoral Thesis, Maastricht University]. Maastricht University. https://doi.org/10.26481/dis.20191128jd

Document status and date:

Published: 01/01/2019

DOI:

10.26481/dis.20191128jd

Document Version:

Publisher's PDF, also known as Version of record

\section{Please check the document version of this publication:}

- A submitted manuscript is the version of the article upon submission and before peer-review. There can be important differences between the submitted version and the official published version of record.

People interested in the research are advised to contact the author for the final version of the publication, or visit the DOI to the publisher's website.

- The final author version and the galley proof are versions of the publication after peer review.

- The final published version features the final layout of the paper including the volume, issue and page numbers.

Link to publication

\footnotetext{
General rights rights.

- You may freely distribute the URL identifying the publication in the public portal. please follow below link for the End User Agreement:

www.umlib.nl/taverne-license

Take down policy

If you believe that this document breaches copyright please contact us at:

repository@maastrichtuniversity.nl

providing details and we will investigate your claim.
}

Copyright and moral rights for the publications made accessible in the public portal are retained by the authors and/or other copyright owners and it is a condition of accessing publications that users recognise and abide by the legal requirements associated with these

- Users may download and print one copy of any publication from the public portal for the purpose of private study or research.

- You may not further distribute the material or use it for any profit-making activity or commercial gain

If the publication is distributed under the terms of Article $25 \mathrm{fa}$ of the Dutch Copyright Act, indicated by the "Taverne" license above, 
Cochlear implantation in adults with early-onset deafness 
(C) Copyright by Joke Debruyne, Maastricht 2019

ISBN: 978-94-6380-546-9

Cover design by Memento Photoprojex, www.mooox.be Layout by Joke Debruyne

Printed by www.proefschriftmaken.nl

The work presented in chapters 2 and 3 of this thesis was supported by a research grant from Cochlear ${ }^{\mathrm{TM}}$. 


\title{
Cochlear implantation in adults with early-onset deafness
}

\author{
PROEFSCHRIFT
}

\begin{abstract}
ter verkrijging van de graad van Doctor aan de Universiteit Maastricht, op gezag van de Rector Magnificus, Prof. dr. Rianne M. Letschert, volgens het besluit van het College van Decanen, in het openbaar te verdedigen

op donderdag 28 november 2019 om 14.00 uur
\end{abstract}

door

Joke Agnes Debruyne 
Promotoren

Prof. dr. B. Kremer

Prof. dr. ir. T. Francart (KULeuven, België)

\section{Copromotor}

dr. ir. J.P.L. Brokx

\section{Beoordelingscommissie}

Prof. dr. W. Mess (voorzitter)

Prof. dr. E. Gerrits (universiteit Utrecht)

dr. E. Mylanus (universiteit Nijmegen)

Prof. dr. H. Kingma

Prof. dr. R. van Oostenbrugge 


\section{Table of Contents}

$\begin{array}{ll}\text { List of Abbreviations } & 7\end{array}$

Chapter 1 General Introduction 9

Chapter 2 Amplitude modulation detection and speech recognition in late- 19 implanted prelingually and postlingually deafened $\mathrm{Cl}$ users

Chapter 3 Fitting prelingually deafened adult cochlear implant users based on electrode discrimination performance

Chapter 4 Systematic review on late cochlear implantation in early deafened adults and adolescents. Part 1: clinical effectiveness

Chapter 5 Systematic review on late cochlear implantation in early deafened adults and adolescents. Part 2: predictors of performance

Chapter 6 Late cochlear implantation in early-deafened adults: a detailed analysis of auditory and self-perceived benefits

Chapter 7 General discussion and valorisation

Summary

Nederlandse samenvatting

References

Dankwoord

203

Curriculum Vitae

209

List of publications 



\section{List of Abbreviations}

$\begin{array}{ll}\text { AMDT } & \text { Amplitude Modulation Detection Threshold } \\ \text { BM } & \text { Basic Map } \\ \text { CI } & \text { Cochlear Implant } \\ \text { CL } & \text { Current Level } \\ \text { CNC } & \text { Consonant Nucleus Consonant } \\ \text { EM } & \text { Experimental Map } \\ \text { EDDL } & \text { Electrode Discrimination Difference Limen } \\ \text { MCL } & \text { Most Comfortable Loudness } \\ \text { MTS } & \text { Monosyllable Trochee Spondee } \\ \text { NCIQ } & \text { Nijmegen Cochlear Implant Questionnaire } \\ \text { pps } & \text { pulses per second } \\ \text { PF } & \text { Prognostic Factor } \\ \text { PTA } & \text { Pure Tone Average } \\ \text { PW } & \text { Pulse Width } \\ \text { rpo } & \text { ripples per octave } \\ \text { SDC } & \text { Smallest Detectable Change } \\ \text { SEM } & \text { Standard Error of Measurement } \\ \text { ST } & \text { Speech Tracking } \\ \text { TMTF } & \text { Temporal Modulation Transfer Function } \\ \text { UCL } & \text { Uncomfortable Loudness } \\ \text { VAS } & \text { Visual Analogue Scale } \\ \text { wpm } & \text { words per minute }\end{array}$



Chapter 1

General introduction 



\section{BACKGROUND}

The first commercially available cochlear implant systems in the 1980's of the previous century were mostly single-electrode devices that used an analogue conversion of sound to electrical current (e.g. House/3M device). When this new technique was applied in adult patients with an early, prelingual ${ }^{1}$ onset of deafness, it became clear that this group reacted differently to electrical stimulation than patients with a late, postlingual ${ }^{2}$ onset of deafness: early deaf patients described odd, tactile sensations or vibrations in and around their head which, very gradually, sometimes following months of stimulation, changed into a sensation which resembled "hearing" (Eisenberg, 1982). With further development of cochlear implant systems, the auditory advancements of early-deafened, adult subjects remained very limited compared to those of postlingually deafened adults (Skinner et al., 1992; Snik, Makhdoum, Vermeulen, Brokx, \& van den Broek, 1997; Waltzman, Cohen, \& Shapiro, 1992). On the other hand, early studies also reported clear positive effects of cochlear implantation in this patient group, such as better sound-awareness, increased independence, and improved communication skills (Hinderink, Mens, Brokx, \& van den Broek, 1995; Zwolan, Kileny, \& Telian, 1996). With the most recent cochlear implant processing techniques, some subjects are able to attain open set speech understanding, but large intersubject variability (Bosco et al., 2013; Caposecco, Hickson, \& Pedley, 2012; O'Gara, Cullington, Grasmeder, Adamou, \& Matthews, 2016; van Dijkhuizen, Beers, Boermans, Briaire, \& Frijns, 2011) and significant differences with postlingually deafened subjects (Kraaijenga et al., 2016; Moon et al., 2012) remain.

In this introduction we continue with a short explanation of how a modern cochlear implant works, followed by what the consequences of an early-onset deafness are for central auditory development. Thereafter we introduce some of the issues we face regarding cochlear implantation in the early-deafened, adult population, and how this thesis aimed to address them.

\section{MECHANISM OF A COCHLEAR IMPLANT}

A cochlear implant is an electronic device that is able to restore hearing in patients with severe sensorineural hearing loss, by means of directly stimulating the auditory nerve fibres in the cochlea. It is made up of an external and an internal part (see Figure 1). The internal part containing the receiver/stimulator and electrode array is implanted surgically, with the electrode array being meticulously placed in the cochlea. The external part, containing the sound processor and transmitter, encodes the sound received by the

\footnotetext{
${ }^{1}$ Onset before the end of the language acquisition period, generally considered about 4-6 years

${ }^{2}$ Onset after the end of the language acquisition period, generally considered about 6 years
} 


\section{Chapter 1}

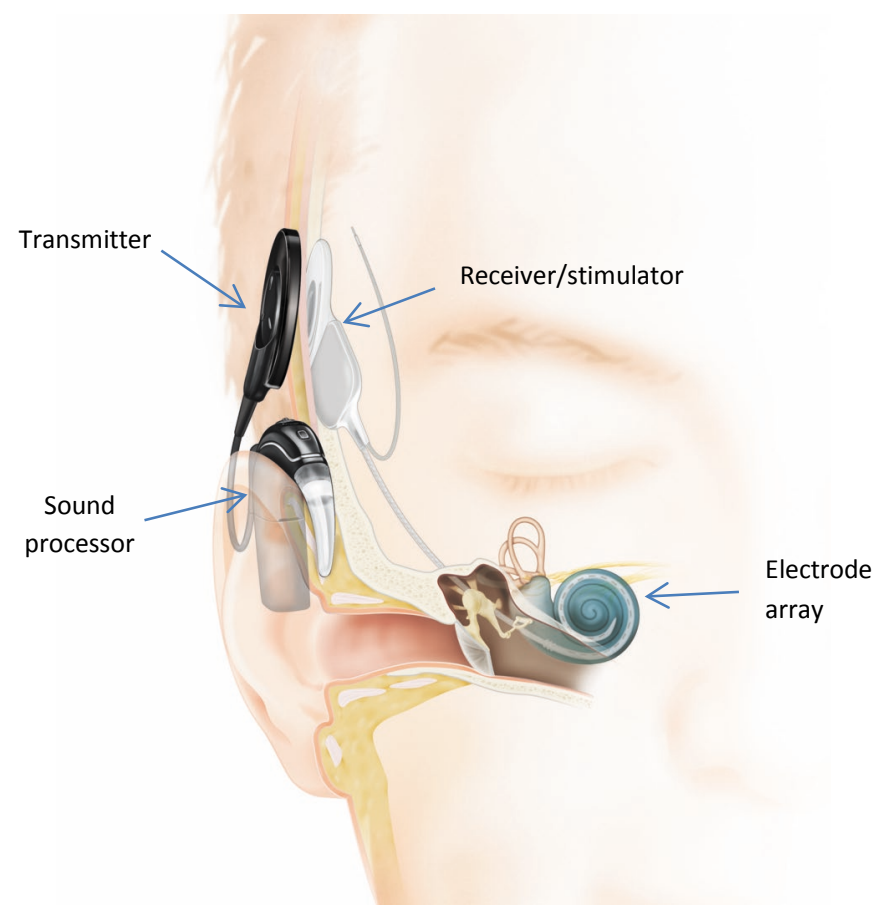

Figure 1. External and internal part of a Cochlear Implant (Copyright Cochlear Limited)

sound processors' microphone. This digital code is transferred from the transmitter to the internal receiver/stimulator, which in its turn converts it to very fast electrical impulses which are sent to the electrode array. These electrical impulses stimulate the auditory nerve fibres in the cochlea, which transfer the signals further to the brain, following the same pathways as in normal hearing. The various auditory nuclei in the brain then translate these impulses into meaningful sounds.

The "language" which is used to translate the sound into a digital code is called the sound processing strategy (see Figure 2 for an overview). Nowadays all sound processing strategies split up the sound picked up by the microphone based on its frequency content, by means of so-called band pass filters. In that way, low-frequency sounds can be transmitted to electrodes located towards the apex (the tip) of the cochlea (e.g. BPF1 in Figure 2), and high-frequency sounds to electrodes at the base (outer end) of the cochlea (e.g. BPF4), following the natural tonotopy ${ }^{3}$ of this auditory structure.

After the sound is filtered into several frequency bands (usually between 12-22, depending on $\mathrm{Cl}$ brand), the temporal fine structure of the acoustic waves is discarded,

\footnotetext{
${ }^{3}$ Tonotopy: spatial organization of anatomic regions in a way that neighbouring frequencies are represented by fysically neighbouring areas; is present throughout the auditory system
} 


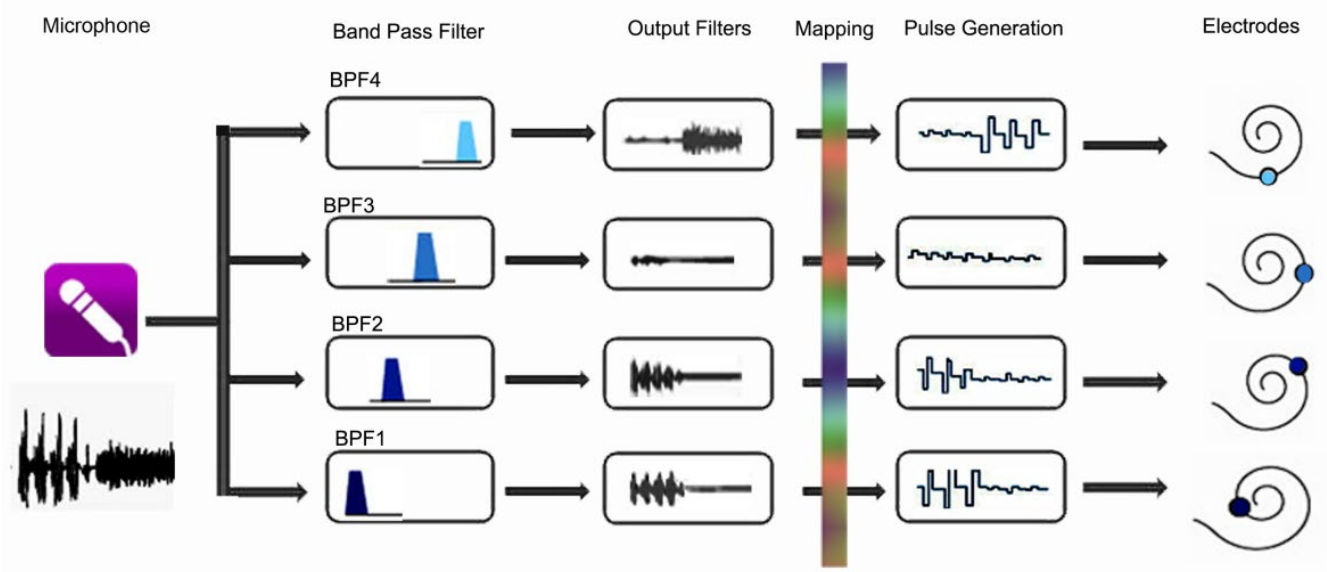

Figure 2. Block diagram of the sound processing of a Cochlear Implant (figure by JPL Brokx)

leaving only the slowly varying temporal envelope. In the next phase, the output is modified in terms of a number of parameters that are individually set for each patient (e.g. the current level that is needed to attain a comfortably loud stimulation). This process is called "mapping". The resulting signal is then used to model fast electrical pulse trains which are passed on to the electrodes.

As a result of this sound processing, information on the frequency content of the acoustic signal is mainly available through the place of stimulation in the cochlea and temporal information is mainly present in the envelope of the $\mathrm{Cl}$ signal. The terms spectral and temporal resolution or processing ability will return on several occassions throughout this thesis, and can be defined as the ability to resolve the frequency components, respectively the changes in auditory information over time, of an acoustic signal.

\section{CONSEQUENCES OF AUDITORY DEPRIVATION ON AUDITORY DEVELOPMENT}

When exposition to auditory information is absent or reduced in early childhood, this has widespread consequences for the maturation of the auditory system. On one hand it has been shown that the connections of the bottom-up auditory pathway, transferring incoming sounds from the auditory nerve fibres in the cochlea through the various auditory nuclei in the brainstem to the auditory cortex, remain at least partly functional in congenital deafness. This allows early-deafened subjects to at least "perceive" sounds when they receive a cochlear implant later in life (Kral, Yusuf, \& Land, 2017). On the other hand, early auditory experience is necessary for the functional development of mainly the auditory cortex. For example, small babies are sensitive to all elementary features of sounds in any language. By the age of 1 , however, they become specialised in those acoustic features that allow them to distinguish phonemes in their own mother tongue and at the same time, they become insensitive to the non-distinctive features. In that way 


\section{Chapter 1}

"auditory objects" are formed: perceptions related to hearing which allow one to categorize the acoustic environment, such as a phonemic categories. In case of early and continued auditory deprivation, a neuronal network incapable to form these auditory objects is developed. When cochlear implantation is performed later in life in an individual with early-onset deafness, the auditory system is thought to no longer have the high synaptic plasticity that allows for this type of bottom-up driven learning (Kral, 2007).

Studies in electrically stimulated congenitally deaf animals have also shown that there is a deviant activation pattern within the primary auditory cortex and a reduced activity of the deeper cortical layers (Kral, 2007). These deep layers are assumed to play a central role in integrating bottom-up, sensory information with top-down information coming from higher-order cortical areas, such as information on active auditory objects. In an experienced brain it is assumed that conflicts between the bottom-up and top-down information streams, i.e. between the sensory input and the brain's expectations, are the driving force behind adult learing (Kral et al., 2017). In case of auditory deprivation, the main hypothesis is that there is a functional decoupling between higher-order and primary auditory areas (Kral, 2007; Kral \& Sharma, 2012), which prevents learning based on these modulating top-down influences, also when auditory input is restored through a cochlear implant.

In addition it has been shown that, secondary to early auditory deprivation and the lack of sufficient auditory input to the higher-order auditory cortex, cross-modal plasticity can occur: the higher order auditory areas are recruited to participate in the functioning of other sensory modalities, such as vision (Kral et al., 2017; Sharma, Campbell, \& Cardon, 2015). The extent to which this occurs might be related to the success of hearing restoration through a cochlear implant: Buckley and Tobey (2011) observed a strong correlation between the amount of visual cross-modal reorganisation and speech understanding scores with $\mathrm{Cl}$ in a group of late-implanted, prelingually deaf subjects. The results of studies investigating the effect of auditory deprivation and subsequent cochlear implantation on cortical auditory evoked potentials (CAEPs), point to the existence of a sensitive period for normal auditory development, which closes at about 7 years of age (Sharma et al., 2015).

In conclusion, when a cochlear implant reintroduces auditory stimulation in earlydeafened patients after the "sensitive period" has closed, i.e. in adolescence or adulthood, the auditory system is unable to adapt sufficiently to the new input and poor outcomes are to be expected. It needs to be noted, however, that most of the earlydeafened subjects in this thesis have not been completely deprived from auditory stimulation during their development, e.g. due to stimulation of their residual hearing with hearing aids and/or a slightly older age at onset of deafness. As a result, the extent of the effects of auditory deprivation as described here might be somewhat reduced in those subjects. 


\section{PROBLEM STATEMENT}

A cochlear implant user needs to be able to extract and process at least part of the spectral and temporal information that is present in the signal delivered by the sound processor, in order to recognize sounds and understand speech in daily life. As described previously, the development of the auditory system alters when auditory stimulation is absent or limited early in life. A substantial part of the limitations in auditory speech understanding experienced by early-deafened $\mathrm{Cl}$ users, can therefore be traced back to the deviant functioning of especially central auditory structures. Much less known, however, is to what extent these early-deafened $\mathrm{Cl}$ users also have problems on a peripheral level, extracting the relevant spectral and temporal information from the $\mathrm{Cl}$ signal. Most research efforts focus on how póstlingually deaf $\mathrm{Cl}$ users perform on measures of spectral and temporal processing (Anderson, Nelson, Kreft, Nelson, \& Oxenham, 2011; Won, Drennan, Nie, Jameyson, \& Rubinstein, 2011). Only a few studies have specifically investigated spectral and temporal processing abilities of early deaf patients (Busby \& Clark, 1996, 2000; Busby, Tong, \& Clark, 1993), generally revealing poorer performance when compared to postlingually deaf patients. An important question is therefore to what extent early-deafened subjects are able to perceive and understand the "language" of the cochlear implant, which allows them to extract the necessary speech cues. More extensive investigation of the spectral and temporal processing abilities of these patients can tell us more on which aspects of sound processing are potentially causing difficulties, and whether changing certain aspects of how the sound is delivered to the early-deafened ear, might help to overcome them.

Clinicians working with early-deafened, late-implanted $\mathrm{Cl}$ users in the field know from general experience that most of these patients have little chance of becoming star performers, defined in terms of open-set, auditory only speech understanding outcomes. They also know that the variation between patients in terms of outcomes is high and that there is a risk for some patients to become non-users, whereas others will be very satisfied despite only limited auditory improvements. Since the majority of cochlear implant clinics have only limited numbers of adult $\mathrm{Cl}$ users with an early-onset deafness, it is difficult to set out clear implantation criteria for new candidates, taking into account both auditory and subjectively experienced outcomes. The latest review on outcomes of this specific population of $\mathrm{Cl}$ users already dates back to 2004 (Teoh, Pisoni, \& Miyamoto, 2004a), thus not incorporating results from studies performed in the last 10-15 years with the most recent technologies. More extensive and up-to-date knowledge on expected outcomes can aid in determining implantation criteria, and is also highly relevant for patient counselling, ensuring realistic expectations. Ideally it would be possible to predict outcomes based on factors that are already known pre-implantation, and thus determining which individuals are likely to benefit most from cochlear implantation. A number of, sometimes large-scale, studies predicting outcomes of postlingually deafened 
$\mathrm{Cl}$ users have been performed in recent years (Blamey et al., 2013; Holden et al., 2013; Lazard, Vincent, et al., 2012; Roditi, Poissant, Bero, \& Lee, 2009). For early-deafened subjects such studies are rare, given the much smaller number of implantations. It can also not simply be assumed that the factors important in predicting outcomes of postlingually deafened $\mathrm{Cl}$ users are the same as for early-deafened $\mathrm{Cl}$ users, given for instance the implications of early onset deafness on auditory development and the variation in early auditory rehabilitation in this population. It would therefore be useful to see whether the outcomes of the available studies can be combined to provide us with useful insights on which factors are important outcome predictors for early-deafened $\mathrm{Cl}$ users.

Finally, when predicting or measuring performance with a cochlear implant, the golden standard is to look at open-set, auditory word or sentence recognition, traditionally in quiet, but more recently also in noise. Part of the early-deafened $\mathrm{Cl}$ users will have chance-level performance on these auditory tests, also after implantation (Caposecco et al., 2012; Heywood, Vickers, Pinto, Fereos, \& Shaida, 2016). However, this does not necessarily imply that these patients do not obtain any auditory benefit from the $\mathrm{Cl}$ stimulation: the traditional tests may simply not be sensitive enough to detect very small changes in auditory perception or enhancements in lip reading. It would thus be useful to see whether there are other, non-traditional tests capable of capturing much smaller changes in auditory performance. From another angle, clinical experience shows that auditory benefit and patient satisfaction do not necessarily go hand in hand. It is therefore interesting to find out in which auditory quality of life areas changes occur after implantation, and whether these changes are also present in patients not obtaining clear gains on traditional auditory tests.

\section{AIMS OF THIS THESIS}

In this thesis we aimed to investigate auditory processing abilities in relation to $\mathrm{Cl}$ fitting, to optimise outcome measurement and to increase knowledge on outcome predictors of cochlear implantation in adult patients with an early onset of deafness.

The following research questions were addressed:

What are the temporal processing abilities of early-deafened $\mathrm{Cl}$ users, and is there a relation with speech understanding?

In Chapter 2 we measured temporal processing abilities of both pre- and postlingually deafened $\mathrm{Cl}$ users by means of their sensitivity to sound-field sinusoidal amplitude modulations. The resulting Amplitude Modulation Detection Thresholds (AMDTs) at different modulation frequencies form a Temporal Modulation Transfer Function (TMTF), which was compared between early- and postlingually deafened $\mathrm{Cl}$ users. It was hypothesized that early-deafened $\mathrm{Cl}$ users would perform less good than postlingually deafened $\mathrm{Cl}$ users. 
Speech perception measures for both patient groups were correlated with the mean AMDT per modulation frequency, the mean AMDT, the attenuation rate of the TMTF and the surface area under the TMTF in order to investigate the possible relationship between performance on the measure for temporal processing and speech understanding skills with $\mathrm{Cl}$.

Do early-deafened $\mathrm{Cl}$ users have limited access to spectral information, and if so, can this be optimised in order to improve performance?

We hypothesized in chapter 3 that early-deafened $\mathrm{Cl}$ users would have difficulties to discriminate between stimulation given on different electrodes, and that this limits their access to the spectral information encompassed in the stimulation pattern of the $\mathrm{Cl}$. We measured electrode discrimination difference limens, i.e. the minimal distance between two electrodes that is needed to perceive them as different, for the entire electrode array in a group of 6 early-deafened $\mathrm{Cl}$ users.

Further in chapter 3, results of the electrode discrimination testing were used in order to create an experimental $\mathrm{Cl}$ fitting containing only discriminable electrodes. Performance with the subjects' clinical fitting was compared to performance with the experimental fitting after an adjustment period of 4 weeks. Performance measurements included tests for speech understanding, both in quiet and in noise, a listening effort test and a spectral ripple discrimination test, which is a measure of spectral resolution. Subjective appreciation of both fittings was assessed by means of a Visual Analogue Scale (VAS) and a questionnaire.

What are the postoperative outcomes of early-deafened, late-implanted $\mathrm{Cl}$ users in terms of auditory performance and (hearing-related) quality of life?

Relevant data with respect to this study aim were first of all gathered through an extensive search of the literature: chapter 4 presents the results of a systematic review including 38 studies that have outcomes on either open- or closed-set speech understanding, audiovisual benefit or subjective benefit. Study outcomes on the relevant outcome domains were extensively analysed, taking into account the results of the thorough quality assessment which identified a number of issues that might have impacted the presented results. Secondly, chapter 6 presents the results of a prospective study, with auditory outcomes of 27 early-deafened $\mathrm{Cl}$ users up to 3 years after implantation.

As a first subquestion, we wondered whether it is possible to capture limited changes in auditory performance after cochlear implantation by means of outcome measures that are better adapted to early-deafened $\mathbf{C l}$ users? Therefore, outcomes in the poorer performing subjects of chapter 6 were assessed not only by a traditional open-set word test, but additionally by means of the conversation-like speech tracking test (De Filippo \& Scott, 1978), as well as the closed-set monosyllable-spondee-trochee (MTS) test (Erber \& Alencewicz, 1976). 
As a second subquestion, we were interested whether benefits for auditory-related quality of life are related to the auditory benefits of early-deafened subjects? Chapter 6 also presents the pre- and postimplantation results on a subjective questionnaire that was developed specifically for early-deafened $\mathrm{Cl}$ users. The relation between subjectively experienced and auditory benefits was assessed by means of calculating correlations between postoperative changes on the questionnaire and changes on the auditory tests.

Which patient-related factors can predict the postoperative speech understanding outcomes of early-deafened, late-implanted $\mathrm{Cl}$ users?

In chapter 5 we present the second part of our systematic review, in which 13 studies with a clear prognostic study goal were identified, investigating a multitude of potential prognostic factors. The outcomes of these studies were combined and analysed in light of potential sources of bias, in order to determine the most promising prognostic factors for speech understanding outcome in this population.

For the study group in chapter 6, we equally investigated which of 8 patient-related factors were related to the 1 year postoperative word recognition scores. The factors showing a significant relation in the simple linear regression analysis, were included in the subsequent multivariable regression analysis, resulting in a final multiple regression model. 


\title{
Chapter 2
}

\section{Amplitude modulation detection and speech recognition in late-implanted prelingually and postlingually deafened $\mathrm{Cl}$ users}

Ear \& Hearing, 2015, 36(5), 557-566. DOI: 10.1097/AUD.0000000000000162

\author{
Anke De Ruiter* \\ Joke Debruyne* \\ Michelene Chenault \\ Tom Francart \\ Jan Brokx
}




\section{ABSTRACT}

Objectives: Many late-implanted prelingually deafened cochlear implant (Cl) patients struggle to obtain open-set speech understanding. Since it is known that low-frequency temporal-envelope information contains important cues for speech understanding, the goal of this study was to compare the temporal-envelope processing abilities of lateimplanted prelingually, and postlingually deafened $\mathrm{Cl}$ users. Furthermore, the possible relation between temporal processing abilities and speech recognition performances was investigated.

Design: Amplitude modulation detection thresholds (AMDTs) were obtained in 8 prelingually and 18 postlingually deafened $\mathrm{Cl}$ users, by means of a sinusoidally modulated broadband noise carrier, presented through a loudspeaker to the $\mathrm{Cl}$ user's clinical device. Thresholds were determined with a 2-down-1-up 3-interval oddity adaptive procedure, at 7 modulation frequencies. Phoneme recognition (Consonant-Nucleus-Consonant) scores (\% correct at $65 \mathrm{~dB} \mathrm{SPL}$ ) were gathered for all $\mathrm{Cl}$ users. For the prelingually deafened group, scores on 2 additional speech tests were obtained: (1) a closed-set monosyllabletrochee-spondee (MTS) test (\% correct scores at $65 \mathrm{~dB}$ SPL on word recognition and categorization of the suprasegmental word patterns), and (2) a speech tracking test (number of correctly repeated words per minute) with texts specifically designed for this population.

Results: The prelingually deafened $\mathrm{Cl}$ users had a significantly lower sensitivity to amplitude modulations than the postlingually deafened $\mathrm{Cl}$ users, and the attenuation rate of their TMTF was greater. None of the prelingually deafened $\mathrm{Cl}$ users were able to detect modulations at 150 and $200 \mathrm{~Hz}$. High and significant correlations were found between the results on the amplitude modulation detection test and CNC phoneme scores, for the entire group of $\mathrm{Cl}$ users. In the prelingually deafened group CNC phoneme scores, word scores on the MTS test, and speech tracking scores correlated significantly with the mean AMDT of the modulation frequencies between 5 and $100 \mathrm{~Hz}$ and with almost all separate amplitude modulation thresholds. High correlations with these speech measures were also found for the attenuation rate of and the surface area below the TMTF. In postlingually deafened $\mathrm{Cl}$ users, CNC phoneme scores only correlated significantly with the $100-$ and $150-\mathrm{Hz}$ amplitude modulation thresholds, as well as with the attenuation rate of and surface area below the TMTF.

Conclusions: Prelingually deafened $\mathrm{Cl}$ users were less sensitive to temporal modulations than postlingually deafened $\mathrm{Cl}$ users, and the attenuation rate of their TMTF was steeper. For all $\mathrm{Cl}$ users, subjects with better amplitude modulation detection skills tended to score better on measures of speech understanding. Significant correlations with low modulation frequencies were found only for the prelingually deafened $\mathrm{Cl}$ users and not for the postlingually deafened $\mathrm{Cl}$ users. 


\section{INTRODUCTION}

While many cochlear implant $(\mathrm{Cl})$ users have excellent speech recognition in quiet (Dorman \& Spahr, 2006), part of this population still struggles (Lazard, Giraud, Gnansia, Meyer, \& Sterkers, 2012; Santarelli, De Filippi, Genovese, \& Arslan, 2008; Teoh et al., 2004a). Most of them are late-implanted prelingually deafened patients, i.e. with an onset of deafness before the end of the language acquisition period. Important information for speech recognition is present in temporal cues up to about $50 \mathrm{~Hz}$ : normal-hearing subjects can achieve nearly perfect speech recognition using slow temporal cues $(<20 \mathrm{~Hz})$, combined with limited spectral information (Friesen, Shannon, \& Cruz, 2005; Shannon, Zeng, Kamath, Wygonski, \& Ekelid, 1995). Since temporal cues seem to be important, and long-term auditory deprivation induces changes along the entire auditory pathway (Teoh, Pisoni, \& Miyamoto, 2004b), the present study was conducted to compare the temporal processing abilities of prelingually and postlingually deafened $\mathrm{Cl}$ users, and to assess whether these abilities are related to speech recognition performance.

A powerful approach to determine the temporal processing abilities of $\mathrm{Cl}$ users is to measure the sensitivity to sinusoidal amplitude modulations. The amplitude modulation detection threshold (AMDT), expressed as the smallest modulation depth that can be detected, measured at several modulation frequencies, results in a temporal modulation transfer function (TMTF). In normal-hearing subjects and postlingually deafened $\mathrm{Cl}$ users the TMTF has a low-pass filter characteristic, i.e. high sensitivity at low modulation frequencies and decreasing sensitivity with increasing modulation frequency (Bacon \& Viemeister, 1985; Dau, Kollmeier, \& Kohlrausch, 1997; Shannon, 1992; Viemeister, 1979).

A range of studies on amplitude modulation detection in $\mathrm{Cl}$ users reported the effects of stimulation rate (Arora, Vandali, Dowell, \& Dawson, 2011; Galvin \& Fu, 2005, 2009; McKay \& Henshall, 2010; Pfingst, Xu, \& Thompson, 2007), stimulation site (Garadat, Zwolan, \& Pfingst, 2012; Pfingst et al., 2007), stimulation mode (Galvin \& Fu, 2005; Pfingst, 2011), stimulus duration (Luo, Galvin, \& Fu, 2010), stimulus level (Galvin \& Fu, 2005, 2009; Pfingst et al., 2007), and loudness growth (McKay \& Henshall, 2010) on AMDTs. In most of these studies the electrical stimulus was presented directly to one or more electrodes using computer-controlled stimulation, bypassing the clinical sound processor. Recently however, Won, Drennan, et al. (2011) and Gnansia et al. (2014) conducted studies in which stimuli were presented in sound-field to the sound processor of postlingually deafened $\mathrm{Cl}$ users. This approach assesses the sensitivity to amplitude modulations in a clinical setting, including both the processors' signal processing and the subjects' individual maps. Won, Drennan, et al. (2011) showed that the TMTFs of $\mathrm{Cl}$ users measured in free-field, have the same low-pass filter shape as the TMTFs of $\mathrm{Cl}$ users measured with electric stimulation directly at one electrode. When they compared the sound-field TMTFs of the postlingually deafened $\mathrm{Cl}$ users to the sound-field TMTFs of normal hearing and hearing-impaired subjects as obtained by Bacon and Viemeister (1985), Won et al. (2011) 
found a lower general sensitivity to amplitude modulation and a steeper slope for the $\mathrm{Cl}$ users compared to the 2 other groups.

Almost all studies regarding temporal processing mentioned so far, investigated the amplitude modulation detection abilities of postlingually deafened $\mathrm{Cl}$ users. However, for prelingually deafened $\mathrm{Cl}$ users the shape of the TMTF is not well characterized. One study, by Busby et al. (1993), obtained electric TMTFs of 3 prelingually deafened $\mathrm{Cl}$ users. All 3 subjects showed a lower sensitivity to amplitude modulation, and for one of these subjects the shape of the TMTF differed from the low-pass filter characteristic; this TMTF had a characteristic of 2 band-pass filters.

Temporal information in speech, e.g. syllabicity, rhythm, manner of articulation, voicing, stress, and intonation, is present in the envelope and periodicity of an acoustic waveform (Rosen, 1992). It is therefore not surprising that several studies have found a correlation between amplitude modulation detection abilities and speech recognition performance for postlingually deafened $\mathrm{Cl}$ users. Cazals, Pelizzone, Saudan, and Boex (1994) found that the rejection rate, which they defined as the difference between the AMDT at the 400and at the $71-\mathrm{Hz}$ modulation frequency, measured at the most apical electrode, correlated with average performance for vowel and consonant recognition administered at 70dBA. Fu (2002) found highly significant correlations between mean electric AMDTs (for a $100 \mathrm{~Hz}$ modulation, averaged over several stimulation levels) and both vowel and consonant recognition scores. In a study by Luo, Fu, Wei, and Cao (2008), mean electric AMDTs (averaged for $20-\mathrm{Hz}$ amplitude modulation across 5 stimulation levels) were significantly correlated with Chinese tone, consonant, and sentence recognition scores. Arora et al. (2011) found that mean AMDTs (obtained with vowel-like stimuli presented via direct audio input to a research processor), predicted sentence in noise outcomes at $65 \mathrm{~dB}$ SPL. These mean AMDTs were determined by averaging across the 50 - and $100-\mathrm{Hz}$ modulation frequencies and various stimulation rates, presented at an acoustic level that when processed through the processor, produced electrical stimulation levels close to the subjects' electrical most comfortable level (MCL). Won et al. (2011) found significant correlations between the mean AMDTs, averaged over 7 modulation frequencies ranging from 10 to $300 \mathrm{~Hz}$, and both consonant-nucleus-consonant (CNC) monosyllabic phoneme scores and speech reception thresholds (SRTs) in noise. When looking the individual modulation frequencies, significant correlations were found only for the higher modulation frequencies (from $75 \mathrm{~Hz}$ onwards for the CNC scores and from $150 \mathrm{~Hz}$ onwards for the SRTs in noise). In addition, Won et al. (2011) found that the attenuation rate, i.e. the slope, of the sound-field TMTF, which is defined as the $b$-component of the exponential function, $A M D T\left(f_{\text {mod }}\right)=-a e^{b f_{\text {mod }}}$ fitted through the AMDTs from 10 to $200 \mathrm{~Hz}$, correlated with both CNC scores in quiet and speech reception thresholds (SRTs) in noise. Given that the attenuation rate is mainly determined by the AMDTs at higher modulation frequencies, the results of Won et al. (2011) suggest that $\mathrm{Cl}$ users with better thresholds for high modulation frequencies obtain better speech understanding scores. In 
a recent study of Gnansia et al. (2014), sound-field AMDTs measured at a low modulation frequency of $8 \mathrm{~Hz}$ also correlated significantly with vowel and consonant identification scores in quiet. In noise, no significant correlations were found (Gnansia et al., 2014). Since in all these studies only postlingually deafened $\mathrm{Cl}$ users were tested, it is unknown whether a correlation exists between amplitude modulation detection measures and speech performance scores for prelingually deafened $\mathrm{Cl}$ users.

The first goal of the present study is to compare the sound-field AMDTs of prelingually deafened $\mathrm{Cl}$ users with the sound-field AMDTs of postlingually deafened $\mathrm{Cl}$ users. It is hypothesized that prelingually deafened $\mathrm{Cl}$ users perform more poorly than postlingually deafened $\mathrm{Cl}$ users on the temporal modulation detection tests. The second goal of this study is to assess the possible relation between temporal processing abilities, measured with AMDTs, and speech recognition scores for the entire group of $\mathrm{Cl}$ users, and for the prelingually and postlingually deafened $\mathrm{Cl}$ users separately.

\section{MATERIALS AND METHODS}

\section{Subjects}

Both prelingually $(n=8)$ and postlingually $(n=18)$ deafened $\mathrm{Cl}$ users participated in this study. All subjects were Dutch native speakers with oral communication as their primary mode of communication. Some of the prelingually deafened $\mathrm{Cl}$ users were also familiar with Dutch sign language. All $\mathrm{Cl}$ users were unilaterally implanted after the age of 18 years and had minimally one year experience with the $\mathrm{Cl}$. The age at onset of deafness for the prelingually deafened subjects can be found in Table 1 . Note that 3 of the prelingually deafened subjects had an onset at 2 or 3 years of age, and might therefore be considered perilingually rather than prelingually deafened. For convenience of comparison however, it was preferred to use the term "prelingual" for all subjects with an onset of deafness before the end of the language acquisition period. The duration of the moderate to profound hearing loss of both the pre- and postlingually deafened $\mathrm{Cl}$ users is also listed in Table 1, together with information about the age at implantation, etiology and implant type. The duration of hearing loss was referred to as the time up to the implantation date, from when the PTA of the best ear was at least $60 \mathrm{~dB} \mathrm{HL}$ or, if this information was not available, the time from when the subject had started to wear hearing aids bilaterally. The use of human subjects was approved by the local Medical Ethical Committee.

\section{Setup}

The acoustic stimuli were presented in a sound-treated booth through a speaker (Klein + Hummel O $110 \mathrm{D}$ ) positioned $1 \mathrm{~m}$ in front of the subjects. The APEX 3 program (developed at ExpORL-K.U.Leuven (Francart et al. 2008)), run on a laptop, was used to present the stimuli in an adaptive procedure. All subjects listened to the stimuli presented in the 
Chapter 2

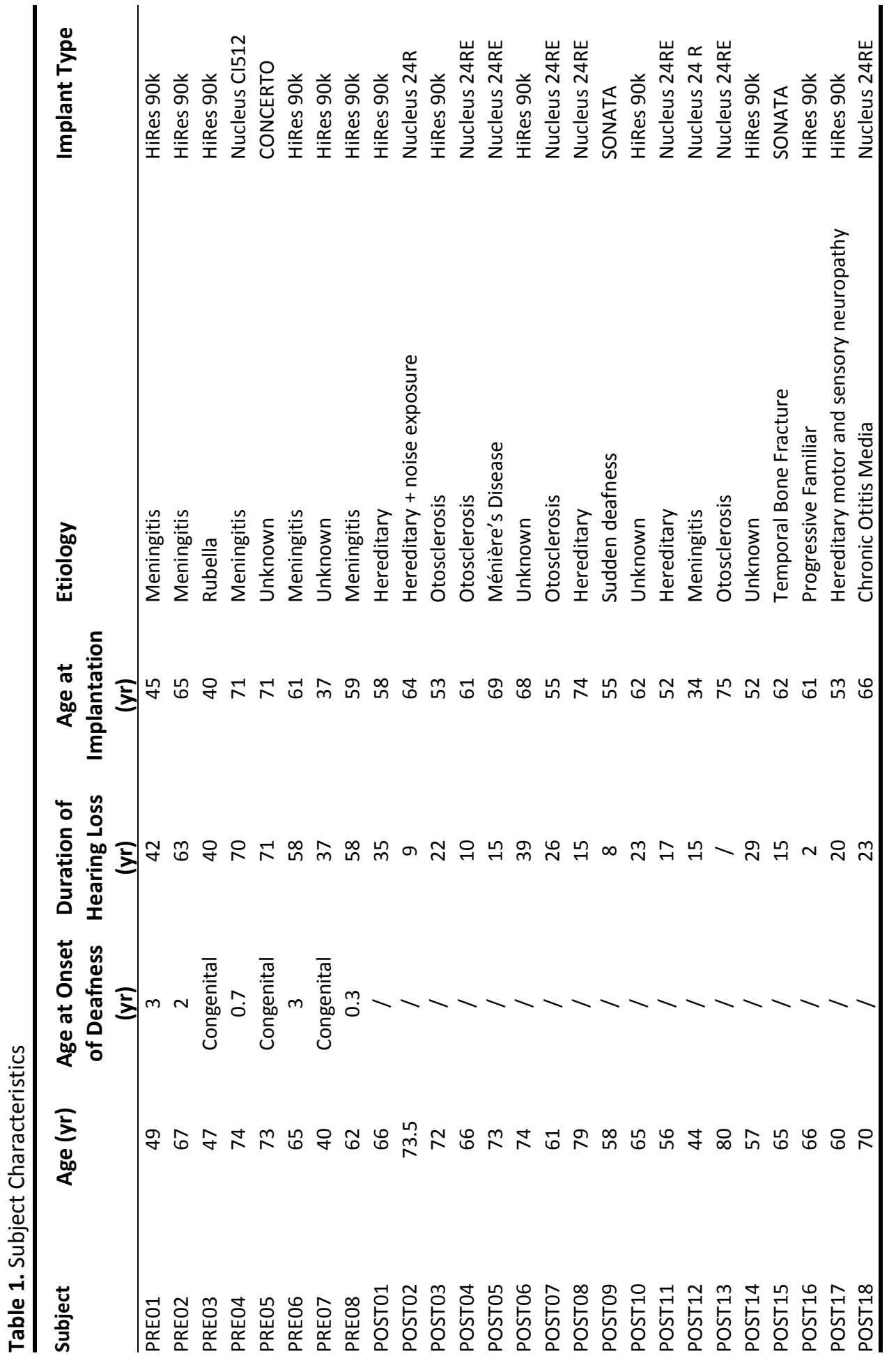


sound-field with their own sound processor and with the clinical map of their own preference.

\section{Stimuli}

Stimuli were generated digitally in MATLAB (The Mathworks, Inc.) with a sampling frequency of $44.100 \mathrm{~Hz}$. A broadband noise carrier was created, which was limited by a fourth-order band-pass filter with cut-off frequencies of 80 and $8500 \mathrm{~Hz}$.

Sound pressure level verification was performed at the position of the head to assure linearity of the setup system. The average sound pressure level of the unmodulated stimulus was $65 \mathrm{~dB}$ SPL. The broadband noise carrier was sinusoidally amplitude modulated by the following equation: $\left.y(t)=f(t)\left[1+m_{i} \sin \left(2 \pi f_{m} t\right)\right]\right]$, where $y(t)$ is the stimulus, $f(t)$ is the broadband noise carrier, $m_{i}$ is the modulation depth and $f_{m}$ the modulation frequency. Seven modulation frequencies were used: $5,10,50,75,100,150$, and $200 \mathrm{~Hz}$. To compensate for acoustic intensity increment due to the amplitude modulation, the modulated signal was divided by the long-term average power of the sinusoidally modulated waveform, $1+\left(m_{i}^{2} / 2\right)$, to equalize the RMS values of the stimuli. Both the modulated and unmodulated stimuli were gated on and off with 30-ms linear ramps. Stimulus duration was $500 \mathrm{~ms}$ and $1000 \mathrm{~ms}$ for an additional test condition administered to a limited number of subjects.

\section{Procedure}

AMDTs were obtained using a 2-down-1-up, 3-interval oddity, adaptive forced-choice procedure, tracking the $70.7 \%$ point of the psychometric function (Levitt, 1971). The stimulus duration as well as the interstimulus duration was $500 \mathrm{~ms}$. Subjects were instructed to choose the stimulus which they perceived as being different from the other two.

The initial modulation depth was $-2 \mathrm{~dB}$ re $100 \%$ amplitude modulation and the initial step size was $4 \mathrm{~dB}$. After 2 reversals the step size was reduced to $2 \mathrm{~dB}$. In a single run 8 reversals were obtained, and the average of the last 6 reversals was used to determine the modulation detection threshold. After completing the runs for all 7 modulation frequencies in a randomized order, the entire session of 7 runs was repeated in order to check for reproducibility. The average of these 2 sessions was taken as the final measure of the modulation detection threshold per modulation frequency. A pause was planned at least once between each session of 7 runs, in order to reduce the possible influence of diminished concentration and fatigue.

\section{Speech Tests}

For both the pre- and the postlingually deafened $\mathrm{Cl}$ users, phoneme scores on an open-set Dutch monosyllabic (CNC) word test (Bosman \& Smoorenburg, 1995) were gathered at 65 $\mathrm{dB}$ SPL. In this test, the phoneme recognition score is measured as a percentage correct. The scores were obtained from the last yearly clinical evaluation of the subject. 
Since prelingually deafened $\mathrm{Cl}$ users generally score poorly on standard open-set word tests, 2 more simple speech tests were also administered to this group. The monosyllabletrochee-spondee (MTS) test, adapted from Erber and Alencewicz (1976), is a Dutch 12item closed-set word identification test, where each word is presented twice. Word scores (entire word should be correct) and suprasegmental scores (number of correct syllables per presented word and the stress pattern of the word should be correct) were gathered as a percentage correct for administration at $65 \mathrm{~dB}$ SPL. The speech tracking test, with texts designed specifically for prelingually deafened Cl users (Boons \& Debruyne, 2011), is an open-set sentence identification test where a number of additional cues (e.g. repeating parts of the sentence, allowing lip reading) is given to the subject in a predetermined order. The amount of time the subject needs to repeat the entire text is used to calculate the score, expressed as the number of words per minute. Speech tests were administered during one of the 2 visits.

\section{Data Analysis}

Normality of AMDTs was checked with the Shapiro-Wilk test. Non-parametric tests were applied in cases of non-normality, which occurred at higher modulation frequency data because of floor effects, and for the attenuation rate data because of outliers. Floor values occurred when modulations were undetected by the participant; in these cases a value of zero was assigned.

To check reproducibility, Spearman correlation coefficients between the first and second measurements of all AMDTs were obtained. A mixed-model was estimated to compare the AMDTs of prelingually and postlingually deafened $\mathrm{Cl}$ users over the 7 modulation frequencies. Additional analyses included independent $t$ tests and Mann-Whitney $U$ tests, depending on normality, to compare the different modulation frequencies pairwise.

Both groups were further compared with respect to the attenuation rate and the surface area below the TMTF. The attenuation rate relates to the shape of the entire TMTF and the surface area below the TMTF relates to the gain (overall sensitivity to amplitude modulation) and shape of the TMTF, whereas the AMDTs only describe the sensitivity to temporal modulations at individual frequencies. The attenuation rate of the TMTF is, as in Won (2011), defined as the $b$-component of an exponential function fitted through the data: $A M D T\left(f_{\text {mod }}\right)=-a e^{b f_{\text {mod }}}$, with $A M D T$ the absolute amplitude modulation detection threshold in $\mathrm{dB}$ re $100 \%$ modulation. Here, $a$ is the intercept, $b$ the attenuation rate, and $f_{\text {mod }}$ the modulation frequency in $\mathrm{Hz}$. The mean fit through the data of both the prelingually and postlingually deafened $\mathrm{Cl}$ users is plotted in Figure 1 . The surface area below the TMTF is calculated as the integral of the exponential function AMDT $\left(f_{\text {mod }}\right)=$ $-a e^{b f}$ mod. An independent $t$ test or Mann-Whitney $U$ test was used to compare the attenuation rate and the surface area below the TMTF between both groups, depending on normality. Bonferroni adjustments occurred separately for the 7 modulation frequencies and the 3 overall AMDT outcome parameters. 
One-sample $t$ tests were performed to investigate the difference between the AMDTs obtained in this study for the postlingually deafened $\mathrm{Cl}$ users and the mean AMDT values reported by Won et al. (2011).

Since floor effects occurred for higher modulation frequencies, Spearman's rank correlation coefficient was used to describe relations between speech performance scores and individual AMDTs, mean AMDTs, the attenuation rate of the TMTF, and the surface area below the TMTF. This was done for the total group of $\mathrm{Cl}$ users and for the pre- and postlingually deafened $\mathrm{Cl}$ users separately. A Bonferroni adjustment for multiple comparisons was added per group for the 7 modulation frequencies and also for the 3 overall AMDT outcome parameters.

Finally, additional measurements were done with 1000 -ms stimuli in all prelingually and 8 of the postlingually deafened $\mathrm{Cl}$ users. To compare the results obtained with both stimulus lengths, a paired sample $t$ test or Wilcoxon Signed Rank test was used, depending on normality.

\section{RESULTS}

Spearman correlation coefficients of the 2 AMDT measurements for each of the 7 modulation frequencies ranged from 0.86 to 0.99 , providing evidence of reproducibility. In Figure 1, the mean sound-field AMDTs of the prelingually and postlingually deafened $\mathrm{Cl}$ users are plotted against the modulation frequency, resulting in sound-field TMTFs. The mean exponential fits of both groups are shown as thin lines in the figure. For comparison, mean sound-field TMTFs of 24 postlingually deafened $\mathrm{Cl}$ users (Won, Drennan, et al., 2011) and of 4 normal-hearing listeners (Viemeister, 1979) are displayed. The low-pass filter shape of the TMTFs of those other studies can also be observed in the mean TMTFs of the prelingually and postlingually deafened subjects tested in this study: sensitivity to amplitude modulation decreased with increasing modulation frequency.

A significant difference was found between the mean sound-field AMDT of the prelingually and postlingually deafened $\mathrm{Cl}$ users $(F=8.69, \mathrm{df}=1,24, p=0.007)$, indicating that prelingually deafened $\mathrm{Cl}$ users are less sensitive to amplitude modulations than postlingually deafened $\mathrm{Cl}$ users. Analysis of the different modulation frequencies showed a significant difference $(p<0.05)$ between both groups for the modulation frequencies 5 to $150 \mathrm{~Hz}$, but not for the $200-\mathrm{Hz}$ modulation frequency, as can be seen in Table 2. After Bonferroni correction for multiple comparisons, however, only the difference for the 100$\mathrm{Hz}$ modulation frequency remained significant. When comparing the numbers relating to the shape of the TMTF, a significant difference was found between pre- and postlingually deafened $\mathrm{Cl}$ users with respect to the attenuation rate $(p=0.021)$, and the surface area below the TMTF $(p=0.003)$; the latter remained significant after Bonferroni correction (see also Table 2). 


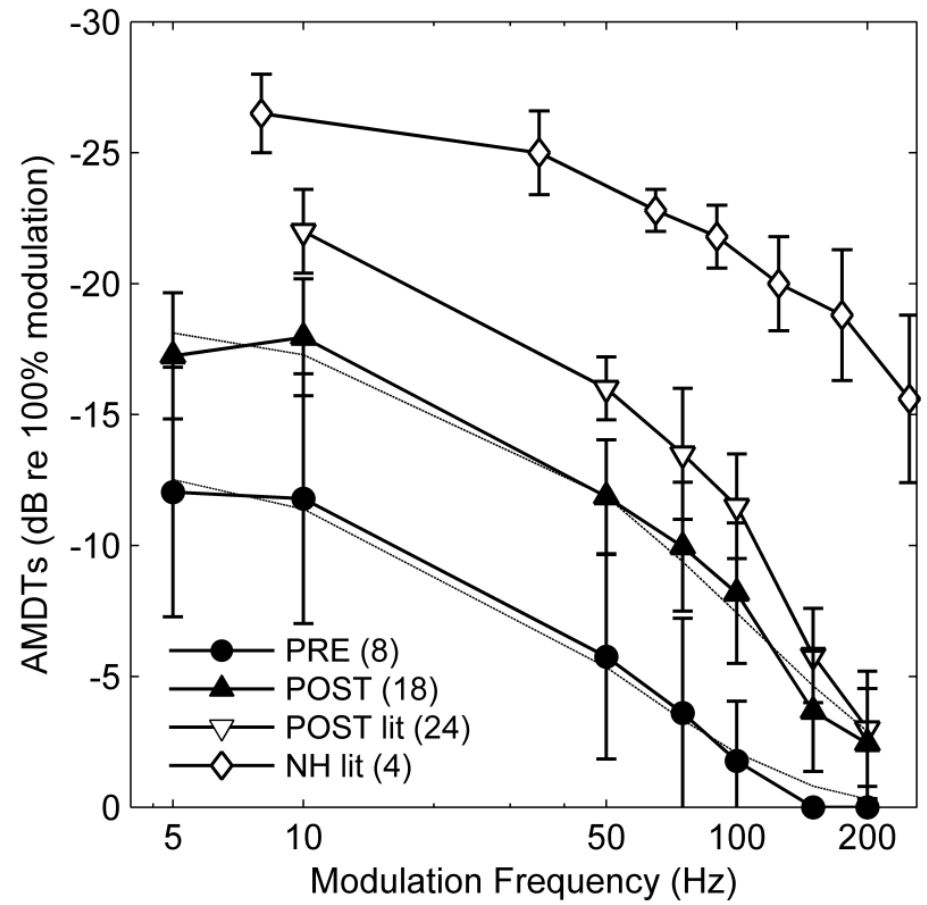

Figure 1. TMTFs based on the mean sound-field AMDTs of the 8 prelingually deafened $\mathrm{Cl}$ users (circles) and the 18 postlingually deafened $\mathrm{Cl}$ users (triangles) measured in this study, as well as $24 \mathrm{Cl}$ users (reverse triangles, data adapted from Won et al. (2011)), and 4 normal-hearing listeners (diamonds, data adapted from Viemeister (1979)). Error bars indicate the 95\% confidence interval; the thin lines are the mean exponential fits for the prelingually and postlingually deafened $\mathrm{Cl}$ users measured in this study.

Table 2. Group Comparisons of Amplitude Modulation Detection Thresholds

\begin{tabular}{llll}
\hline & $\begin{array}{l}\text { Prelingual } \\
\text { (Mean } \pm \text { SD) }\end{array}$ & $\begin{array}{l}\text { Postlingual } \\
\text { (Mean } \pm \text { SD) }\end{array}$ & $p$ \\
\hline $5 \mathrm{~Hz}$ & $-12.04 \pm 6.88$ & $-17.24 \pm 5.21$ & $0.044^{*}$ \\
$10 \mathrm{~Hz}$ & $-11.79 \pm 6.88$ & $-17.95 \pm 4.82$ & $0.015^{*}$ \\
$50 \mathrm{~Hz}$ & $-5.75 \pm 5.63$ & $-11.86 \pm 4.70$ & $0.008^{*}$ \\
$75 \mathrm{~Hz}$ & $-3.60 \pm 5.22$ & $-9.96 \pm 5.33$ & $0.00 *^{*}$ \\
$100 \mathrm{~Hz}^{\ddagger}$ & $-1.77 \pm 3.29$ & $-8.18 \pm 5.81$ & $0.00 *^{+}$ \\
$150 \mathrm{~Hz}^{\ddagger}$ & $0.00 \pm 0.00$ & $-3.67 \pm 4.98$ & $0.013^{*}$ \\
$200 \mathrm{~Hz}^{\ddagger}$ & $0.00 \pm 0.00$ & $-2.43 \pm 4.56$ & 0.08 \\
\hline mean AMDT & $-7.0 \pm 4.71$ & $-10.8 \pm 6.05$ & $0.007^{*}$ \\
attenuation rate, $b \ddagger$ & $-0.039 \pm 0.029$ & $-0.012 \pm 0.005$ & $0.021^{*}$ \\
surface area & $543 \pm 485$ & $1636 \pm 881$ & $0.00 *^{+}$ \\
\hline Amplitude Malion
\end{tabular}

Amplitude Modulation Detection Thresholds expressed in dB re $100 \%$ Modulation

*: significant with $\alpha<0.05$

$\dagger$ : significant after Bonferroni correction

¥: Mann-Whitney $U$ test was used instead of independent-samples t-test 
The individual sound-field TMTFs of the prelingually deafened $\mathrm{Cl}$ users are shown in Figure 2. Two prelingually deafened $\mathrm{Cl}$ users (PREO1 and PRE04) scored in the range of the postlingually deafened $\mathrm{Cl}$ users, while one of the subjects (PRE02) was unable to distinguish modulated from unmodulated stimuli at any of the 7 modulation frequencies. In addition, none of the prelingually deafened $\mathrm{Cl}$ users were able to detect amplitude modulations at modulation frequencies of 150 and $200 \mathrm{~Hz}$. Three of the 8 subjects (38\%) were able to detect amplitude modulations at $75 \mathrm{~Hz}$ and 2 of the $8(25 \%)$ at $100 \mathrm{~Hz}$, as shown in Figure 3. When a subject was unable to detect amplitude modulations at a certain modulation frequency, the threshold was reported as $0 \mathrm{~dB}$ re $100 \%$ modulation. Of the 18 postlingually deafened $\mathrm{Cl}$ users in this study, the percentage of subjects that was able to distinguish modulated from unmodulated stimuli decreased with increasing modulation frequency: $89 \%$ of subjects could detect modulations at $100 \mathrm{~Hz}, 61 \%$ at $150 \mathrm{~Hz}$ and $44 \%$ at $200 \mathrm{~Hz}$ (see Figure 3). The mean sound-field TMTF of the postlingually deafened $\mathrm{Cl}$ users in this study indicated a lower overall sensitivity to amplitude modulation when compared to the mean sound-field TMTF of the $24 \mathrm{Cl}$ users tested by Won, Drennan, et al. (2011), as can be seen in Figure 1. Significant differences between both groups were found for the modulation frequencies 10 to $100 \mathrm{~Hz}(p<0.05)$. AMDTs at $5 \mathrm{~Hz}$ could not be com-pared since this modulation frequency was not tested by Won et al. (2011). No significant differences were found between the AMDTs at the modulation frequencies 150 and $200 \mathrm{~Hz}$.

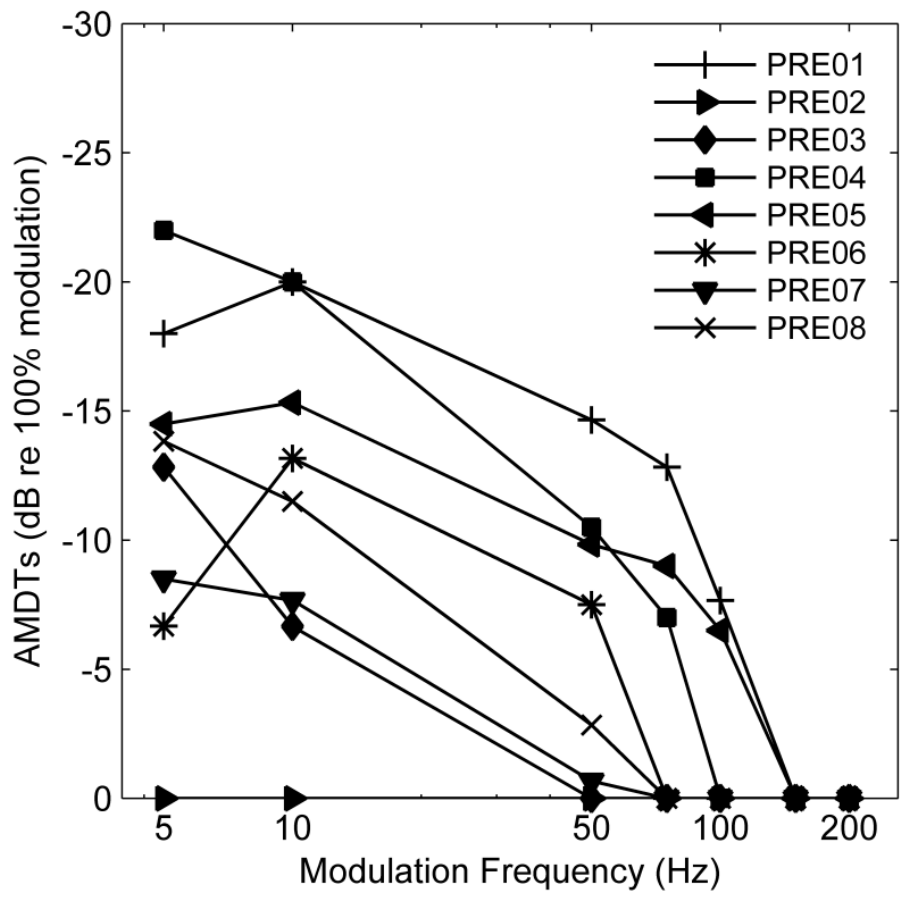

Figure 2. Individual freefield TMTFs of 8 late implanted prelingually deafened $\mathrm{Cl}$ users. 


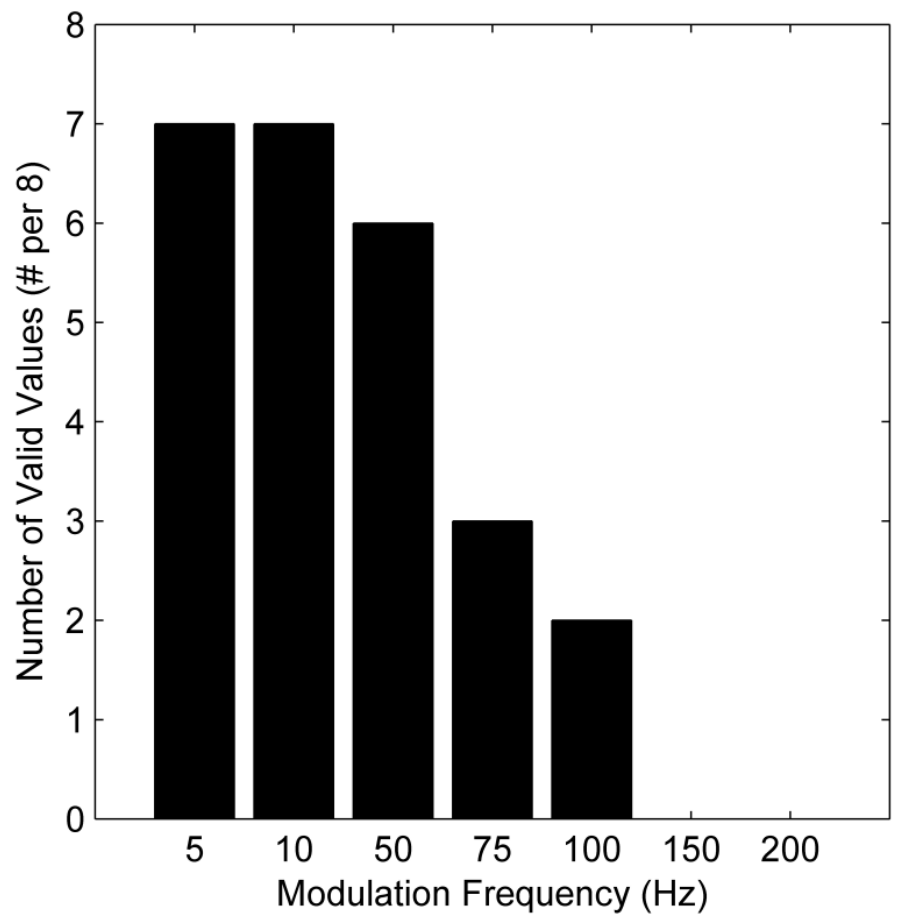

Figure 3. The number of late implanted prelingually deafened $\mathrm{Cl}$ users that were sensitive to that modulation frequency.

Table 3 presents correlations (Spearman's Rho) between the temporal modulation detection abilities and various speech scores (CNC phoneme scores, MTS words scores, MTS suprasegmental scores, and speech tracking), for the prelingually and postlingually deafened group, as well as for the total group of subjects. The means and standard deviations of the speech tests are also given for each group. Note that for the MTS-test and the speech tracking test, scores are only available for the prelingually deafened $\mathrm{Cl}$ users.When looking at all the $\mathrm{Cl}$ users tested in this study, the correlations between the CNC phoneme scores and the thresholds for the individual modulation frequencies, the mean AMDT, the attenuation rate of and the surface area below the TMTF, were significant $(p<0.05)$, as can be seen in the last column of Table 3. After Bonferroni correction for multiple comparisons, only the correlations with the $5-, 10-$, and $200-\mathrm{Hz}$ modulation frequencies were no longer significant.

For the prelingually deafened $\mathrm{Cl}$ users separately, significant correlations $(p<0.05)$ were found between the individual modulation frequencies $5,10,50,75$, and $100 \mathrm{~Hz}$ and CNC phoneme scores, MTS word scores, and speech tracking scores. Correlations with MTS suprasegmental scores were not significant, except for the $100-\mathrm{Hz}$ modulation frequency. The mean AMDT of this group was significantly correlated with all speech measures (see Table 3). Finally, the attenuation rate of the TMTF and the surface area below the TMTF 


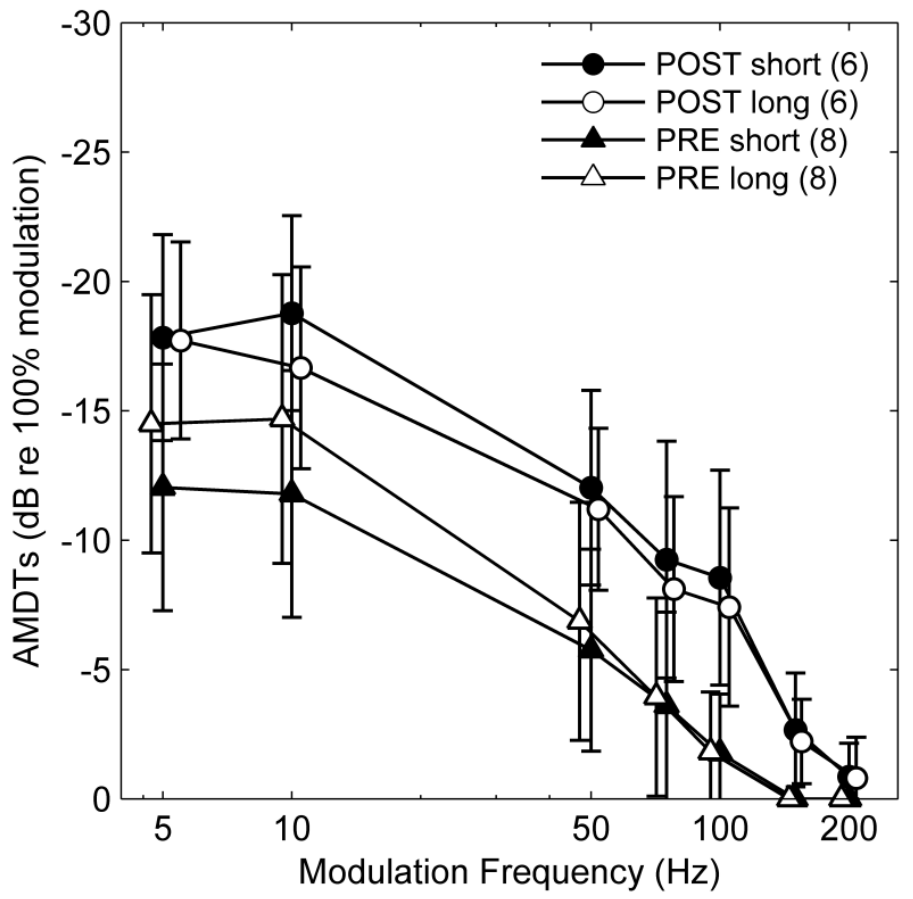

Figure 4. Mean free field TMTFs obtained with short stimuli (filled symbols) and long stimuli (open symbols) of 8 prelingually (circles) and 6 postlingually deafened $\mathrm{Cl}$ users (triangles). Error bars indicate the 95\% confidence interval.

were significantly correlated with all speech measures, except for the correlation between the attenuation rate and the MTS suprasegmental score. Only a limited number of correlations remained significant after the correction for multiple comparisons was applied (see Table 3).

When looking at the postlingually deafened $\mathrm{Cl}$ users, significant correlations were found between CNC phoneme scores and the $100-$ and $150-\mathrm{Hz}$ modulation frequencies, as well as the attenuation rate of the TMTF. These correlations remained significant after Bonferroni correction (see Table 3).

In Figure 4, the graphs represent the mean TMTFs for the 8 prelingually and 6 of the 18 postlingually deafened subjects, in 2 test conditions: a short $(500 \mathrm{~ms}$ ) versus a long (1000 $\mathrm{ms}$ ) stimulus duration. The prelingually deafened $\mathrm{Cl}$ users demonstrated a higher sensitivity to $5-\mathrm{Hz}(p=0.005$, significant after Bonferroni correction) and $10-\mathrm{Hz}(p=0.011$, significant with $p<0.05)$ amplitude modulations obtained with the 1000 -ms stimuli in comparison to the 500-ms stimuli. For the 6 postlingually deafened $\mathrm{Cl}$ users, there was no significant difference for the pairwise comparisons between AMDTs obtained with 500- or 1000-ms stimuli at any modulation frequency. 


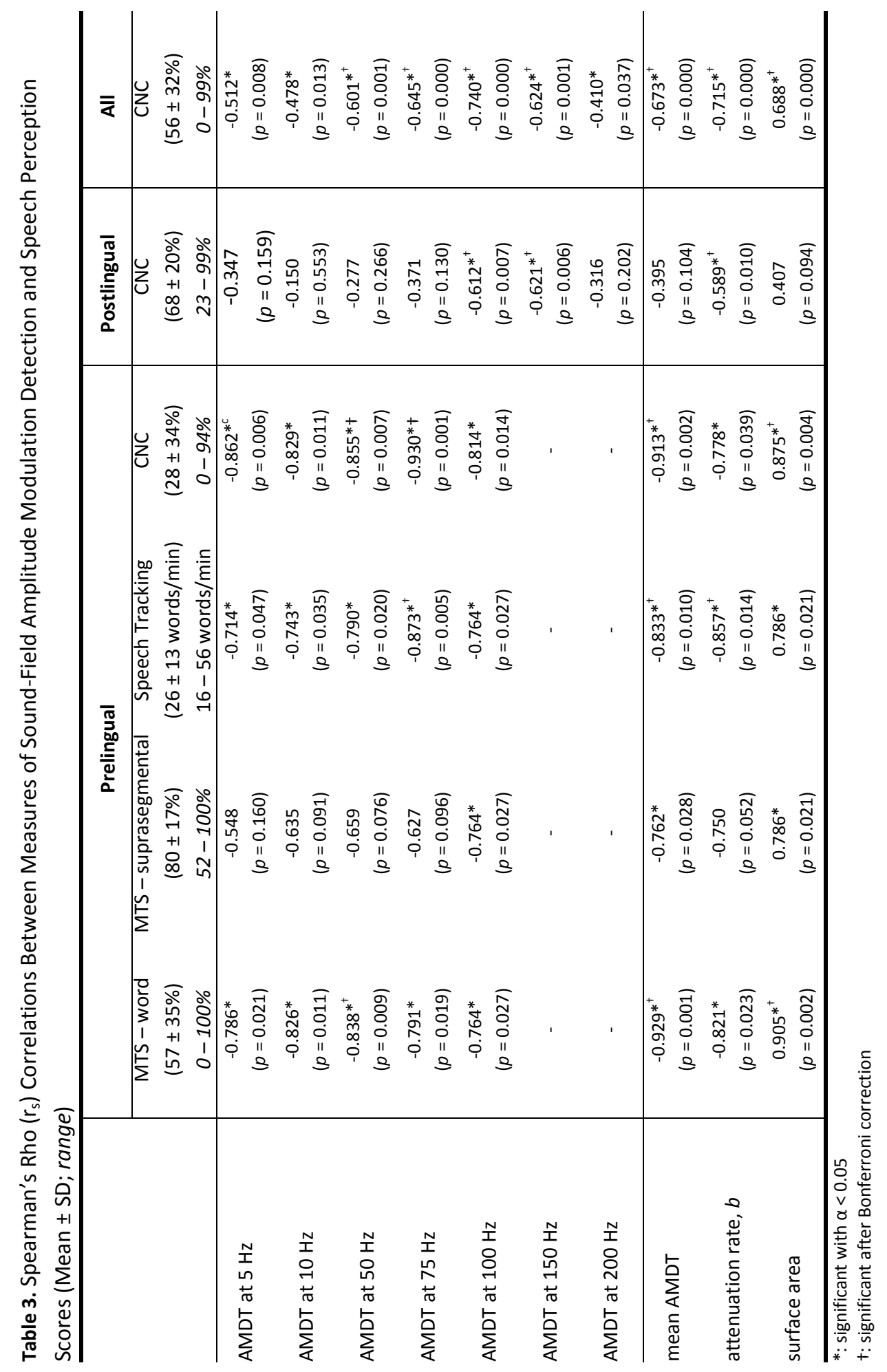




\section{DISCUSSION}

\section{The TMTF of Prelingually and Postlingually Deafened $\mathrm{CI}$ Users}

The first goal of the study was to compare the sound-field AMDTs of prelingually and postlingually deafened $\mathrm{Cl}$ users. The absolute sensitivity of prelingually deafened $\mathrm{Cl}$ users to amplitude modulation was significantly lower than that of the postlingually deafened $\mathrm{Cl}$ users. The modulation frequency where the difference remained significant, even after correction for multiple comparisons, was $100 \mathrm{~Hz}$. This might be interpreted as such that below $100 \mathrm{~Hz}$, the prelingually deafened $\mathrm{Cl}$ users are still reasonably capable of detecting amplitude modulations, as can also be found in Figure 3. At $100 \mathrm{~Hz}$, their performances start to decline very quickly, whereas most of the postlingually deafened $\mathrm{Cl}$ users still perform adequately. Above $100 \mathrm{~Hz}$, however, $\mathrm{Cl}$ users in both groups start to have great difficulties, resulting in smaller group differences again. In addition, both the attenuation rate of the TMTF and the surface area below the TMTF differed significantly between both groups. Although not meeting the stricter criterion for multiple comparisons, it does point to a trend that the slope of the TMTF of the prelingually deafened $\mathrm{Cl}$ users is steeper, thus that performances in this group declined more rapidly towards the higher modulation frequencies.

These findings are in agreement with Busby et al. (1993), who found lower sensitivity to electrical amplitude modulation in 3 prelingually deafened $\mathrm{Cl}$ users than in 4 postlingually deafened $\mathrm{Cl}$ users. A likely explanation is that the changes along the entire auditory pathway, due to the early onset and long-term auditory deprivation (Teoh et al., 2004b), contributed to a reduced sensitivity to amplitude modulation in prelingually deafened $\mathrm{Cl}$ users.

The individual TMTFs of 2 prelingually deafened $\mathrm{Cl}$ users (PRE01 and PRE04), as can be seen in Figure 2, lay within the 95\% - confidence interval of the postlingually deafened $\mathrm{Cl}$ users, while one subject (PRE2) was unable to distinguish any of the modulated stimuli from the unmodulated stimuli. In Table 1 it can be seen that there are no striking differences between the etiologies of these subjects. A possible explanation for these interindividual differences could be that better performing prelingually deafened $\mathrm{Cl}$ users had a larger amount of residual hearing (mostly at low frequencies) preoperatively, or had this during a longer period in their lives. Residual hearing, especially at $500 \mathrm{~Hz}$, and age at onset of severe to profound hearing loss are both good predictors for $\mathrm{Cl}$ outcome (Blamey et al., 2013; Lazard, Vincent, et al., 2012). To evaluate this theory, the mean preoperative hearing thresholds of the 8 prelingually deafened $\mathrm{Cl}$ users in this study at 250 and $500 \mathrm{~Hz}$, were compared to their mean AMDT, but no significant correlation was found. Also, no correlation between the mean AMDT and the age at onset of deafness was found for the prelingually deafened $\mathrm{Cl}$ users.

The individual sound-field TMTFs of the prelingually deafened $\mathrm{Cl}$ users in this study all had a low-pass filter characteristic. The band-pass filter shape of the TMTF, as found in one 
prelingually deafened $\mathrm{Cl}$ user by Busby et al. (1993), was not found in this study. However, for some subjects the AMDT at the $5-\mathrm{Hz}$ modulation frequency was lower than at the 10$\mathrm{Hz}$ modulation frequency. A similar observation was done by Viemeister (1979), who found lower modulation sensitivity for short stimulus durations (250 and $500 \mathrm{~ms}$ ) in combination with slow modulation frequencies $(<8 \mathrm{~Hz})$ in normal-hearing listeners, for a gated broadband noise carrier. When they applied continuous stimuli with the same durations and modulation frequencies, the sensitivity was comparable with the threshold at the $10-\mathrm{Hz}$ modulation frequency again. The authors hypothesize that the effect might be due to interferences from the gating, which consequently "mask" some of the modulations. More recently, there are no studies where a gated broadband noise stimulus was used in combination with modulation frequencies below $10 \mathrm{~Hz}$ (except for Gnansia (2014), but no TMTF was determined there).

The sound-field AMDTs of the 18 postlingually deafened $\mathrm{Cl}$ users were compared to those of the $24 \mathrm{Cl}$ users evaluated by Won et al. (2011), as shown in Figure 1. At the 10- to 100$\mathrm{Hz}$ modulation frequencies, the measured AMDTs in this study were significantly worse than those of Won et al. (2011). Since it is known that preoperative factors, such as duration of severe to profound hearing loss, duration of moderate hearing loss (up to the onset of severe to profound hearing loss), and the PTA of the better ear have a significant influence on speech performance with $\mathrm{Cl}$ (Blamey et al., 2013; Lazard, Vincent, et al., 2012), it was investigated whether such differences between the subjects in this study and the subjects in the study of Won et al. (2011) could account for the lower sensitivity to modulations found in this study. Although no information about the preoperative residual hearing of the subjects of Won et al. (2011) is available, there is no reason to assume that preoperative PTAs would be different between both groups. The CNC scores of both groups could not be compared, since phoneme scores were measured in this study and word scores by Won et al. (2011). The duration of the moderate to severe hearing loss before implantation could be compared, although no details are given by Won et al. (2011) as to how the "duration of hearing loss" is defined. It was found that the "duration of hearing loss" of the subjects in the study of Won et al. (2011), was significantly shorter $(p=0.033)$ than of the subjects in this study. This shorter duration of hearing loss could contribute to the better AMDTs found by Won et al. (2011).

Another difference between both studies lies in the adaptive procedure: in this study a $3 A F C$ procedure was used, versus a 2AFC procedure in Won et al. (2011). It is known that with a $2 A F C$ procedure, threshold estimates show a larger variability due to smaller values of the sensitivity index d' (Hacker \& Ratcliff, 1979; Leek, Hanna, \& Marshall, 1992). Although this might have contributed to the significant differences that were found, it cannot explain them.

Finally, whereas in this study stimuli of 500 ms were used, Won et al. (2011) used stimuli of $1000 \mathrm{~ms}$. The reason why the shorter duration might have an influence pertains to the effect of short, gated carriers on low-frequency slow-rate modulation (Viemeister, 1979), 
as mentioned above. For the prelingually deafened $\mathrm{Cl}$ users, the improvement of the sensitivity to $5-$ and $10-\mathrm{Hz}$ modulations for $1000-\mathrm{ms}$ stimuli compared to $500-\mathrm{ms}$ stimuli, might be an illustration of this phenomenon. The additional measurements that were done with 1000-ms stimuli in 6 of the postlingually deafened $\mathrm{Cl}$ users, however, showed that the longer stimulus duration had no effect. It is not clear why this effect was found only for the prelingually deafened $\mathrm{Cl}$ users, but it must be concluded that stimulus duration did most likely not contribute to the differences with the results of Won et al. (2011).

\section{The Relation between Sensitivity to Amplitude Modulation and Speech Performance}

The second goal of the present study was to determine the possible relation between the ability to detect amplitude modulations and speech recognition scores, for the entire group as well as for prelingually and postlingually deafened $\mathrm{Cl}$ groups separately.

When looking at all $\mathrm{Cl}$ users from both groups together, all correlations with $\mathrm{CNC}$ phoneme scores and AMDT parameters were significant. For the individual modulation frequencies 5, 10 and $200 \mathrm{~Hz}$, the correlations were no longer significant after multiple comparisons correction, which is consistent with the weak correlations that are found for these modulation frequencies in the postlingually deafened group.

In the group of prelingually deafened $\mathrm{Cl}$ users, the MTS word scores, the speech tracking scores, and the CNC phoneme scores had high and significant correlations with the separate modulation frequencies $(5,10,50,75$, and $100 \mathrm{~Hz})$, the mean threshold across modulation frequencies and the measures relating to the shape of the TMTF. Even though not all separate correlations met the strict multiple comparisons criterion, the high correlation coefficients suggest a relation between the variables. This may indicate that this group was able to utilize modulations in the speech envelope up to $100 \mathrm{~Hz}$ for the identification of segmental information (CNC phoneme scores and MTS word scores), and running speech (speech tracking). Correlations with the MTS suprasegmental scores were less high and mostly not significant, which may be partly due to ceiling effects, since suprasegmental scores were generally high (up to 100\%) and the standard deviation was smaller than that of the other speech scores.

For the postlingually deafened $\mathrm{Cl}$ users, significant correlations were found between the CNC phoneme scores and AMDTs at the higher modulation frequencies of $100 \mathrm{~Hz}, 150 \mathrm{~Hz}$, and with the attenuation rate and the surface area below the TMTF, but not with individual AMDTs at 5, 10,50, 75, or $200 \mathrm{~Hz}$, or the mean AMDT. This was unexpected, given that it is known from literature with normal hearing subjects that especially lowfrequency temporal cues contain important information for speech recognition when spectral cues are limited. Moreover, additional temporal information above approximately $20 \mathrm{~Hz}$ does not even seem to be used as long as minimal spectral cues are available (Friesen et al., 2005; Shannon et al., 1995). Also in contrast with this finding is the fact that mainly the slowly-varying envelope, with frequency components up to about $250 \mathrm{~Hz}$, is 
encoded in current commercial cochlear implant sound processing strategies (McDermott, McKay, \& Vandali, 1992; Vandali, Whitford, Plant, \& Clark, 2000).

The presence of a ceiling effect for the low modulation frequencies in this subject group, which could give rise to the absence of correlations, is not very likely since the standard deviations for these results were not particularly smaller for the postlingually deafened $\mathrm{Cl}$ users (see Table 2).

Looking at the literature, however, these findings in the postlingually deafened group are in agreement with the results of Won et al. (2011), who found significant correlations between CNC phoneme scores and the AMDTs at higher modulation frequencies (75 to $300 \mathrm{~Hz}$ ) but not with AMDTs at low modulation frequencies (10 and $50 \mathrm{~Hz}$ ). Also in agreement with this study, they found significant correlations between the attenuation rate and CNC phoneme scores, where the attenuation rate, the $b$-component of the exponential fit, was primarily determined by the AMDTs at higher modulation frequencies (i.e. 200 and $300 \mathrm{~Hz}$ ). The latter is also the case for the data in this study, where correlations with the attenuation rate were only significant for the modulation frequencies 50 to $200 \mathrm{~Hz}$. Taken together, our current results and the study of Won et al. (2011) both suggest that when $\mathrm{Cl}$ users have better amplitude modulation detection skills at higher modulation frequencies, they attain better speech understanding scores. Though only a $100-\mathrm{Hz}$ modulation frequency was measured, and stimulation was done directly at the electrodes, Fu (2002) found highly significant correlations between the mean AMDT (averaged over various stimulus levels) and vowel and consonant recognition scores. On the other hand, a number of studies found correlations with lower modulation frequencies. Also using electric stimulation, Luo et al. (2008) found a significant correlation between AMDTs at both the $100-\mathrm{Hz}$ and $20-\mathrm{Hz}$ modulation frequency and consonant- and sentence recognition scores, but not vowel recognition scores. Recently, Gnansia et al. (2014) found a significant correlation between sound-field AMDTs at a modulation frequency of $8 \mathrm{~Hz}$ and consonant and vowel identification scores.

\section{Further Considerations}

\section{Sound-field Stimulation}

The sound-field TMTF can be seen as a representation of temporal performance in the daily life situation, when the $\mathrm{Cl}$ is used. It describes the characteristics of the auditory system combined with the $\mathrm{Cl}$, including speech coding strategy and individual map settings. Since the attack time of a noise reduction system is relatively long compared to the stimulus duration, no effect of this feature is assumed. Compression is another factor that could affect the AMDT, however, Won et al. (2011) tested the influence of AGC on AMDTs in $7 \mathrm{Cl}$ users and did not find significant effects at $65 \mathrm{~dB}(\mathrm{~A})$. In general though, it is difficult to control the modulation depth without stimulating directly at the electrode(s), especially when different speech coding strategies and different individual map settings are used. 


\section{Intensity and Loudness Cues}

In this study, it is unlikely that intensity cues were used by the subjects to detect amplitude modulations, since a compensation for intensity increase coming with amplitude modulation was executed, as described in the methods section. However, it is possible that loudness cues were used, since loudness is more related to the peakintensity than the RMS of the signal (Fraser \& McKay, 2012; McKay \& Henshall, 2010). This could give the subject an additional cue to choose between modulated and unmodulated stimuli.

Loudness balancing and roving are ways to compensate for possible loudness cues. In research regarding amplitude modulation, the effect of these interventions has been investigated. McKay and Henshall (2010) performed loudness balancing of 250- and 500$\mathrm{Hz}$ modulated stimuli and measured loudness differences. The effect on AMDTs was not measured. They concluded that modulated stimuli were perceived as louder than unmodulated stimuli. Fraser and McKay (2012) measured the effect of balancing and roving in $4 \mathrm{Cl}$ users. For the low modulation frequency $(50 \mathrm{~Hz}), 2$ out of $4 \mathrm{Cl}$ users showed worse AMDTs after balancing and roving. For the higher modulation frequencies (300-600 $\mathrm{Hz}$ ), this effect was found in 3 out of 4 subjects. Galvin, Fu, Oba, and Baskent (2014) measured AMDTs at 10 and $100 \mathrm{~Hz}$ in nine $\mathrm{Cl}$ users, with and without a novel method to control for possible loudness cues. In an adaptive task, the stimuli were balanced and global roving was applied. The AMDTs were generally worse with this method, but controlling for loudness cues did not affect the general finding that AMDTs became worse when the modulation frequency increased. In another study with $5 \mathrm{Cl}$ users, Chatterjee and Oberzut (2011) found that there was a small but significant effect of roving (without loudness balancing) on the overall gain of the TMTF. The shape of the TMTF, however, was unaffected. On the other hand, Won et al. (2011) found that roving had no significant effect on AMDTs, as measured for modulation frequencies of 10,100 , and $200 \mathrm{~Hz}$ in $2 \mathrm{Cl}$ users. In conclusion, it was found that when applying roving, AMDTs were generally worse, but the overall shape of the TMTF was not affected. For sound-field stimulation though (see Won et al., 2011), these findings have not yet been confirmed.

In the current study, loudness balancing between the modulated and unmodulated stimuli was not performed. This task would be very difficult for the prelingually deafened $\mathrm{Cl}$ users, especially for the low modulation frequencies, where changes in loudness are clearly noticeable during the stimulus. However, when no balancing is performed, an even larger amount of roving should be applied in order to correct for possible loudness cues, especially when modulation depths are larger than $-15.92 \mathrm{~dB}$ re $100 \%$, which is $>16 \%$ (Chatterjee \& Oberzut, 2011). The latter occurs for most of the modulation frequencies of the prelingually deafened $\mathrm{Cl}$ users (Figure 2). Since in this study neither roving nor loudness balancing was applied, the AMDTs might overestimate the sensitivity to amplitude modulations due to loudness cues, and this for the whole range of modulation frequencies. This means that there is a small, but unlikely, chance that the real AMDTs, 
and thus also the gain of the TMTFs, might be lower. In addition, this effect of loudness cues could be different for each subject group. If this were the case, this would have an impact on the results discussed in the previous sections, regarding the lower sensitivity of the prelingually deafened $\mathrm{Cl}$ users to amplitude modulations, and the correlations between the speech measures and the individual and mean AMDTs. However, since it is also known that the shape of the TMTF would not be affected, this would not change the results where the main parameter was the attenuation rate. In other words, the significant difference that was found between the attenuation rate of the TMTF of the postlingually and prelingually deafened $\mathrm{Cl}$ users, and the high correlations between the attenuation rate and various speech measures in all groups, would not likely have been influenced by loudness cues.

\section{CONCLUSIONS}

This study measured the temporal processing abilities of both prelingually and postlingually deafened $\mathrm{Cl}$ users by means of the sensitivity to sound-field sinusoidal amplitude modulations of a broadband noise. It was found that prelingually deafened $\mathrm{Cl}$ users were less sensitive to amplitude modulations than postlingually deafened $\mathrm{Cl}$ users, and that their performance degraded more quickly with increasing modulation frequency. High correlations were found between temporal modulation detection and speech recognition ability in both pre- and postlingually deafened $\mathrm{Cl}$ users. Better modulation detection thresholds that degraded less quickly when the modulation frequency increased, were related to better speech understanding scores. For postlingually deafened $\mathrm{Cl}$ users, such correlations were not found between modulation frequencies below $100 \mathrm{~Hz}$ and speech recognition. Although this has been observed in literature before, it is not clear what causes this effect, given that primarily slowly varying temporal cues are used for speech recognition.

Finally, although the influence of loudness cues on the absolute levels of the AMDTs cannot be ruled out, the significant correlations that were equally found with the shapedependent measures of the TMTF, point to the authenticity of these findings. 
Chapter 3

\title{
Fitting prelingually deafened adult cochlear implant users based on electrode discrimination performance
}

\author{
International Journal of Audiology, 2017, 56(3), 174-185.
}

DOI:10.1080/14992027.2016.1243262

\author{
Joke Debruyne \\ Tom Francart \\ Miranda Janssen \\ Kim Douma \\ Jan Brokx
}




\section{ABSTRACT}

Objective: This study investigated the hypotheses that (1) prelingually deafened $\mathrm{Cl}$ users do not have perfect electrode discrimination ability and (2) the deactivation of nondiscriminable electrodes can improve auditory performance.

Design: Electrode discrimination difference limens were determined for all electrodes of the array. The subjects' basic map was subsequently compared to an experimental map, which contained only discriminable electrodes, with respect to speech understanding in quiet and in noise, listening effort, spectral ripple discrimination and subjective appreciation.

Study Sample: Subjects were six prelingually deafened, late implanted adults using the Nucleus cochlear implant.

Results: Electrode discrimination difference limens across all subjects and electrodes ranged from 0.5 to 7.125 , with significantly larger limens for basal electrodes. No signifcant differences were found between the basic map and the experimental map on auditory tests. Subjective appreciation was found to be significantly poorer for the experimental map.

Conclusions: Prelingually deafened $\mathrm{Cl}$ users were unable to discriminate between all adjacent electrodes. There was no difference in auditory performance between the basic and experimental map. Potential factors contributing to the absence of improvement with the experimental map include the reduced number of maxima, incomplete adaptation to the new frequency allocation, and the mainly basal location of deactivated electrodes. 


\section{INTRODUCTION}

Prelingually deafened patients who receive a cochlear implant $(\mathrm{Cl})$ in adulthood, after many years of severe hearing impairment or auditory deprivation, constitute a very specific group of cochlear implant users. In the early years of cochlear implantation, results from this population were not very promising, with subjects experiencing very limited benefit with respect to speech understanding (Snik et al., 1997; Tong, Busby, \& Clark, 1988; van den Broek, Admiraal, Brokx, Mens, \& Spies, 1992). With advancements in speech processing strategies, these subjects have generally become more successful $\mathrm{Cl}$ users, albeit with large inter-individual differences (Caposecco et al., 2012; Teoh et al., 2004a). In view of this substantial variability in outcome, the current study attempted to optimize cochlear implant performance for prelingually deafened subjects by means of individually adapted cochlear implant fitting.

When looking at the underlying auditory processing abilities of this patient population, it is clear that the long-term auditory deprivation of these subjects has influenced the development of the auditory pathways. A long period of profound deafness will severely reduce the number of spiral ganglion cells on a peripheral auditory level. A clear relation between the number of surviving spiral ganglion cells and clinical performance with a cochlear implant has not yet been shown, however (Fayad \& Linthicum, 2006; Xu, Kim, Snissarenko, Cureoglu, \& Paparella, 2012). Anomalies in this part of the auditory system alone can therefore not fully explain the limited results for most prelingually deafened, late-implanted $\mathrm{Cl}$ users. It is more likely that this can be explained by aberrant development of the auditory brainstem and, most of all, the auditory cortex. Studies in children using cochlear implants (Gordon et al., 2011) and animal models (Butler \& Lomber, 2013) have shown that the subcortical nuclei of the auditory brainstem require auditory input for further maturation; a sophisticated tonotopy, for instance, does not develop (Butler \& Lomber, 2013). Synaptic activity in the primary auditory cortex will deviate substantially from normal including less activation of the deeper cortical layers (Kral, 2007). These deep layers are usually responsible for cognitive modulation, integrating descending (top-down) input from higher-order cortical layers, and also project back to subcortical structures. Based on animal studies, Kral (2007); Kral, Tillein, Heid, Hartmann, and Klinke (2005) hypothesized that a functional decoupling between the primary auditory cortex and higher cortical layers occurs when auditory input is not restored before the end of a sensitive period which, in humans, is thought to lie around 7 years of age (Sharma, Nash, \& Dorman, 2009). This may lead to an auditory system that is incapable of forming auditory objects, which is a requirement for attributing meaning to incoming auditory stimuli (Kral, 2013). In addition, cross-modal reorganization may occur, with mainly higher order auditory structures being recruited by other sensory modalities such as vision (Kral, 2007). This phenomenon is illustrated by Buckley and Tobey (2011), who showed that higher activation in the auditory cortex in response to peripheral visual 
movements was correlated with lower speech understanding scores in $\mathrm{Cl}$ users with a prelingual onset of deafness. In conclusion, differences in auditory processing may explain the reduced speech understanding performance as compared to postlingually deafened $\mathrm{Cl}$ users.

Given the irreversible consequences of long-term auditory deprivation in prelingually deafened $\mathrm{Cl}$ users, arranging the speech signals through the processing strategies might facilitate the reception and processing in the compromised auditory structure. Due to the filterbank system of $\mathrm{Cl}$ processing, which relies on cochlear tonotopy, the exact pattern of stimulated sites along the array contains potentially important information for speech understanding. It is hypothesized in this study that it is difficult for prelingually deafened $\mathrm{Cl}$ users to discriminate between stimulation given on different electrodes, and that this limits their access to the spectral information encompassed in the stimulation pattern. These difficulties might be due to a reduced neural survival on a peripheral level, with different electrodes stimulating largely the same neurons, as well as to a less precise development of tonotopy throughout the auditory system. Additionally, the auditory cortex might encounter more difficulties in translating the incoming neural signals into distinct, auditory percepts. The ability to discriminate between stimulation given at different sites in the cochlea can be evaluated by means of electrode discrimination testing. In postlingually deafened cochlear implant users, (near) perfect discrimination was found when stimulation was given at C-level (Chatterjee \& Yu, 2010; Laneau \& Wouters, 2004; Zwolan, Collins, \& Wakefield, 1997). To our knowledge, two studies have specifically tested the electrode discrimination abilities of prelingually deafened $\mathrm{Cl}$ users. In the first study (Busby \& Clark, 1996), 50\% discrimination limens of six subjects for an apical, mid and basal electrode ranged from 0.5 to over 7 electrodes. In the second study, average limens across 3 electrode positions targeting $70.7 \%$ correct, varied from 0.68 to 5.36 electrodes in a group of 16 young subjects (Busby \& Clark, 2000).

Electrode discrimination results and speech understanding have been found to correlate in some, but not all studies investigating this relationship (Busby \& Clark, 2000; Henry, McKay, McDermott, \& Clark, 2000; Zwolan et al., 1997). However, these studies did not use up-to-date speech coding strategies. More recently, studies using spectral ripple discrimination tests generally found good correlations between discrimination thresholds and speech understanding (Drennan, Anderson, Won, \& Rubinstein, 2014; Jones, Won, Drennan, \& Rubinstein, 2013; Won, Drennan, \& Rubinstein, 2007). Spectral ripple discrimination relies on complex spectral pattern analysis, and therefore is expected to require good electrode discrimination skills. Moreover, a number of studies have shown no further improvement in speech understanding with more than about 7 to 12 active electrodes (Friesen et al., 2005; Shannon, Cruz, \& Galvin, 2011). Our objective was therefore to determine whether speech understanding performance of prelingually deafened $\mathrm{Cl}$ users would improve when only mutually discriminable electrodes were selected for use in cochlear implant fitting. Selectively turning off electrodes related to 
"ineffective sites" in order to improve speech perception is referred to as the "site selection strategy" (Pfingst, Burkholder-Juhasz, Zwolan, \& Xu, 2008). To our knowledge, only two studies have tested speech recognition in postlingually deafened subjects using an experimental cochlear implant program containing only discriminable electrodes. In a study by Zwolan et al. (1997), seven out of nine subjects showed improvement with the experimental map. It is important to note that these subjects made use of the MPEAK strategy, an F0F1F2-based strategy encoding the fundamental frequency (F0), first (F1) and second (F2) formant, no longer used nowadays. A study by Saleh, Saeed, Meerton, Moore, and Vickers (2013) found significant improvements in speech perception scores for 16 of 25 postlingually deafened subjects, using an experimental program that contained either only discriminable or the two-thirds most discriminable electrodes.

The first goal of the present study is to determine electrode discrimination difference limens for the entire electrode array in a number of prelingually deafened, late-implanted adult cochlear implant users. It is hypothesized that a number of electrodes have less than perfect discrimination limens, with subjects not being able to discriminate between stimulation on adjacent or even further removed electrodes. The second goal is to investigate whether changing $\mathrm{Cl}$ fitting based on the electrode discrimination results, can lead to improved speech understanding, listening effort, spectral ripple discrimination and subjective appreciation in this patient population.

\section{METHODS}

\section{Subjects}

The six subjects in this study met the following inclusion criteria: onset of deafness/severe hearing loss before or at the age of 4 years, unilateral implantation with a Nucleus cochlear implant, a minimum of 1 year experience with their implant, Dutch native language, oral communication as primary mode of communication, age at implantation $>16$ years, age at inclusion $>18$ years and $<80$ years and normal reading skills. Four subjects used a Nucleus CP810 processor, one used a CP910 processor and one a Freedom processor. Their PTA (pure-tone average of 500, 1000 and $2000 \mathrm{~Hz}$ ) for the contralateral ear ranged from 92 to $115 \mathrm{~dB}$ HL. Table 1 presents the subjects' main characteristics. The Medical Ethical Committee of the Maastricht University Medical Center granted approval for the study protocol.

\section{Electrode discrimination testing}

Prior to electrode discrimination measurements, thresholds (T-levels), most comfortable loudness (MCL) levels, and uncomfortable loudness (UCL) levels were determined for a number of electrodes, and loudness level was balanced between electrodes. Deactivated electrodes in the map that subjects used in daily life, were not included in the experiments. 
Stimuli were pulse trains with a duration of $1 \mathrm{~s}$, a rate of 900 pulses per second (pps), pulse width (PW) 25 or $50 \mu \mathrm{s}$ with a $8 \mu$ s interphase gap. 900 pps was also the clinically used rate for all subjects. T-levels were determined on five electrodes spread over the array and interpolated for the remaining electrodes. The MCL-level was measured on the most central electrode of the array. Initial MCL-levels for the remaining electrodes were set at the same percentage of the dynamic range (between T-and UCL-level) as the central electrode. UCL-levels were determined for all active electrodes of the array. For both the MCL- and UCL-levels a loudness scale containing five steps (almost inaudible, soft, medium, loud, too loud) was used, with the MCL-level corresponding to "medium" and the UCL-level to "too loud".

All electrodes were loudness balanced pairwise for loudness with their neighboring electrode. The most central electrode of the array was chosen as a starting point in order to minimize the drift in loudness towards the ends of the array. Neighboring electrodes were chosen because they are easier to balance due to the small differences in sound quality and because it was important for the discrimination task that nearby electrodes were well balanced. In each run, the current level (CL, as clinically applied by Cochlear $^{\mathrm{TM}}$ ) of the reference electrode was kept constant and the level of the comparison electrode was varied adaptively in a 1 up - 1 down procedure (Levitt, 1971). The starting level for each comparison electrode and the first, central, reference electrode was the initial, previously determined, MCL-level. Each trial consisted of two intervals, one on the reference electrode and one on the comparison electrode, using the same stimuli as described above, with an interstimulus interval of $1 \mathrm{~s}$. The subject judged which of both stimuli was louder after each trial. The level of the comparison electrode was then adapted accordingly in the next trial. A step size of $5 \mathrm{CL}$ was applied with the stimulation level on any electrode maximized at the predetermined UCL-level. The run was terminated after six reversals; the average of the last four reversals was used to calculate a "balanced level". Two runs were conducted per electrode pair and the average balanced level of both runs was used as the new fixed level of the reference electrode for the next pairwise comparison. It is possible that small differences in loudness may remain between "balanced" stimuli; therefore, loudness roving was adopted to prevent the effect of loudness from systematically impacting the results (see below).

Electrode discrimination testing was performed using a 2-down-1-up 3-interval oddity adaptive procedure (Levitt, 1971), converging to the $70.7 \%$ correct point on the psychometric function. In other words, a difference limen of 1 electrode would indicate that the subject could correctly discriminate adjacent electrodes with an accuracy of $70.7 \%$. Loudness roving was applied to each interval of the stimulus presentation, the magnitude being calculated per subject, based on the principles of Dai and Micheyl 
$(2010)^{4}$. In each trial, the subject was presented with three stimuli in random order: two on the same, fixed (reference) electrode and one on the other (comparison) electrode. The interstimulus interval was $0.5 \mathrm{~s}$. The electrode number of the comparison electrode was varied adaptively in a 2-down-1-up manner. At the beginning of the procedure the comparison electrode was five electrode numbers more basal (for electrode numbers 7 to 22) or apical (for electrode numbers 1 to 6 ) than the reference electrode. The switch from a basal to an apical direction resulted in some overlap, in particular in the vicinity of reference electrode numbers 6 and 7. For S5, the distance at the beginning of the procedure was increased, to maximally nine electrodes, due to discrimination difficulties. The procedure started with a step size of two electrodes for the first two reversals, and a step size of one electrode for the remaining four reversals. The average of the last four reversals was used as the result of the run. When the subject showed a perfect performance between adjacent electrodes ( $100 \%$ accuracy), the reversals in the procedure would be between the same and the neighboring electrodes, leading to an average difference limen of 0.5 . Two runs were performed for each reference electrode. The electrode discrimination difference limen (EDDL) was then calculated as the difference between the reference electrode number and the average of both runs.

\section{Creating the experimental map}

An experimental map (EM) was programmed, starting from the subjects' basic map (BM) used by the subject in daily life. Using the results of the electrode discrimination testing, electrodes were deactivated so that all remaining electrodes were mutually discriminable for the subject. If there was more than one possible deactivation pattern, the number of deactivated electrodes was kept as low as possible with the remaining electrodes spread optimally over the array. In case of contradictory discrimination in apical versus basal direction, the best discrimination result was used. The Frequency Allocation Table (FAT) was redistributed over the remaining electrodes using the "recalculate" option of the Custom Sound ${ }^{\mathrm{TM}}$ software ${ }^{5}$. Since this induces a shift of the tonotopic representation of the frequency map, subjects were given a habituation period of four weeks with the EM. At the end of the study, the processor was reprogrammed according to the subjects' preference.

The ACE strategy was applied in the BM of all subjects, with the number of maxima set to eight. In the EM, the ratio between maxima and number of electrodes was kept at the

\footnotetext{
${ }^{4}$ For our adaptive procedure, the roving range was calculated as the averaged difference between the balanced levels of the two runs of all electrode pairs, which is an estimation of the amount of error after the balancing procedure, divided by .69. If, for example, the average difference between the results of run 1 and run 2 for a subject was $4 \mathrm{CL}$, the roving range $\mathrm{R}$ needed to be at least $5.8 \approx 6 \mathrm{CL}$. This roving range was then applied on each side of the balanced loudness level of each electrode: if the balanced MCL-level was $150 \mathrm{CL}$, the loudness was randomly roved between 147 and $153 \mathrm{CL}$ for that electrode.

${ }^{5}$ With this algorithm, the channel frequency boundaries are redistributed in a way that they increase linearly up to $1 \mathrm{kHz}$ and logarithmically above $1 \mathrm{kHz}$, with relatively narrow apical channels and broader basal channels.
} 

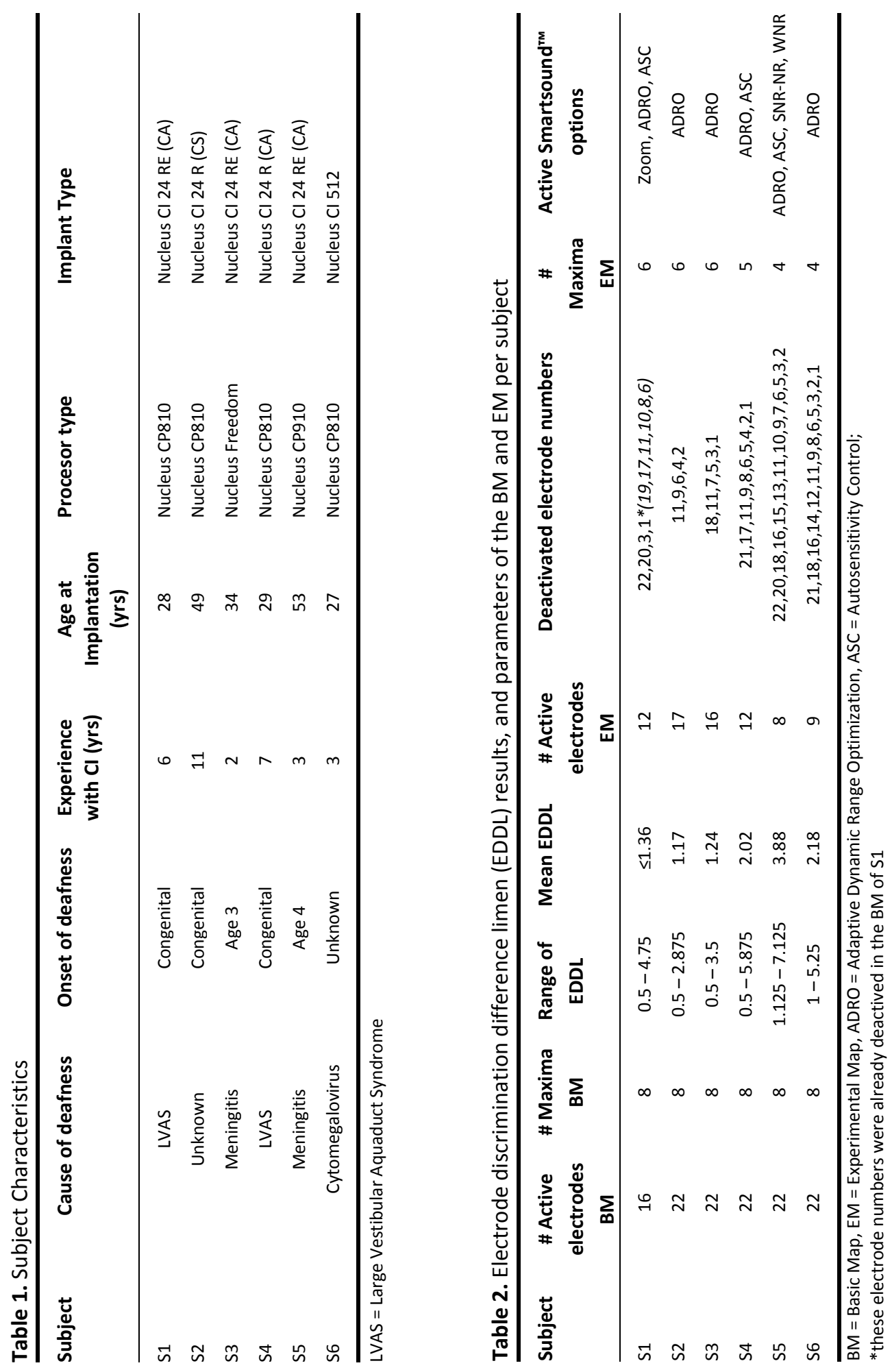
original level, thereby reducing the number of maxima, with a minimum of four. This reduction of the maxima was preferred in an attempt to emphasize spectral contrasts, given that the number of electrodes available for stimulation was reduced. If SmartSound ${ }^{\mathrm{TM}}$ options were used, they were programmed in combination with the EM as well, since denying subjects the use of their favourite SmartSound ${ }^{\mathrm{TM}}$ options could have a negative impact on their acceptance of the new map. Only during the spectral ripple discrimination test, which is described below, SmartSound ${ }^{\mathrm{TM}}$ options were deactivated. All remaining parameters were left unaltered in the EM, except when subjects indicated that the EM was louder or softer, in which case a general adjustment of C-levels was allowed.

\section{Evaluation procedures}

Tests on speech understanding, listening effort, spectral resolution and a questionnaire addressing subjective appreciation were administered to compare the EM with the BM in a repeated-measures design with 2 visits, separated by a four week habituation period with the EM.

\section{Speech understanding tests}

Phoneme scores in \% correct on an open set, Dutch, monosyllabic (CNC) word test (Bosman \& Smoorenburg, 1995) were collected at 75, 65 and 55 dB SPL. A Dutch open-set sentence test (LIST) (van Wieringen \& Wouters, 2008) was administered in quiet at $65 \mathrm{~dB}$ SPL. If the score in quiet was larger than $50 \%$, administration of the LIST was done in noise as well, using an adaptive procedure. If, on the other hand, the average score on the LIST in quiet was lower than $50 \%$, or if the result on the LIST in noise was worse than $10 \mathrm{~dB}$ SNR, a closed-set Dutch number test (LINT) (van Wieringen \& Wouters, 2008) was added to the test battery. If the score on the LINT in quiet was greater than $50 \%$, administration was also performed in noise. In addition, LIST and LINT scores were determined as the average of three adaptive runs.

\section{Listening effort test}

If the LIST or LINT had been administered in noise, a subjective test of self-reported listening effort was added to assess more subtle differences in speech perception, at a supra-threshold level. Such differences might be experienced by subjects, but are likely to remain undetected when using only standard speech recognition tests. In this test, the subject needed to indicate listening effort on a 13-point scale, ranging from "no effort" to "very much effort". The speech material used was either the LIST or the LINT, depending on the subject, presented at 6 different signal-to-noise ratios $(-6,-3,0,+3,+6$ and $+9 \mathrm{~dB})$. The signal-to-noise ratio as determined with the BM was taken as the reference (0) and was left unchanged in the second session with the EM.

\section{Spectral ripple discrimination test}

In the spectral ripple discrimination test, the subject was asked to discriminate between a spectrally rippled noise stimulus (a noise with sinusoidal variations in amplitude along the frequency axis) and the same stimulus shifted in phase (positions of the spectral peaks and 
valleys reversed). The spectral ripple discrimination test is considered to be a test of spectral resolution, which is supported by the correlations with other measures of spectral resolution, including spatial tuning curves (Anderson et al., 2011) and measures of channel interactions (Jones et al., 2013; Won, Jones, Drennan, Jameyson, \& Rubinstein, 2011). Our hypothesis was that spectral resolution, as assessed with the ripple discrimination test, improves with the experimental map. This is based on the reasoning that when indiscriminable electrodes are deactivated and all channels elicit different percepts, small differences in the spectral pattern across channels can be readily discerned.

For the spectral ripple stimuli, a Gaussian broadband noise carrier was used (120 - 7680 $\mathrm{Hz}$ ), spectrally modulated on a log-frequency axis, as in Anderson et al. (2011). Ripple densities of $0.125,0.176,0.250,0.354,0.500,0.707,1.000,1.414,2.000,2.828,4.000$, 5.657 and 8.000 ripples/octave (rpo) were created, with the peaks equally spaced on the log-frequency axis. The spectral modulation depth was held constant at $30 \mathrm{~dB}$. The starting phase was $0^{\circ}$ for the standard and $180^{\circ}$ for the inversed stimuli. The duration of the stimuli was $500 \mathrm{~ms}$, including a $30 \mathrm{~ms}$ Gaussian rise/fall time. Stimuli were presented in sound-field at $60 \mathrm{~dB} S P L$ and $a \pm 4 \mathrm{~dB}$ random level rove was added to avoid the influence of loudness cues. A psychophysical experiment was used to find the spectral ripple discrimination threshold per subject, which is the highest ripple density at which the subject can still discriminate two spectrally rippled stimuli with inverse positions of the peaks and valleys. Two standard and one inverse stimuli were presented per trial, in a 2down-1-up 3-interval oddity adaptive procedure (Levitt, 1971). Each run started with a ripple density of 0.176 . The mean of the last six out of ten reversals was used to establish the threshold, averaged over three runs.

It is known that for ripple densities above 2.000 ripples per octave, the ripple pattern is no longer clearly represented in the processor's output (Croghan, Krishnamoorthi, \& Smith, 2013). Spectrogram analysis of our own physical measurements confirmed this, as can be seen in Figure 1 for ripple densities 1.000, 2.000 and 4.000 rpo. We therefore did not expect subjects being able to discriminate between phase-inversed ripples when ripple densities were high.

\section{Questionnaire}

Subjective appreciation of both the BM and the EM was evaluated with a questionnaire consisting of 17 questions regarding primary sound processing, sense of safety and ease of communication. The questionnaire was completed at the beginning of each session and concluded with a Visual Analogue Scale asking subjects to score their general appreciation of the program, whether it be BM or EM. In the second session subjects designated which program they preferred (BM, EM or no preference).

\section{Materials}

The determination of T-, MCL- and UCL-levels was performed using the subjects' own speech processor and Cochlear's clinical software Custom Sound ${ }^{\mathrm{TM}}$. For the loudness 
balancing and electrode discrimination testing, individual electrodes were stimulated with a L34 research processor in combination with Cochlear NIC research software and the APEX test platform (Francart, van Wieringen, \& Wouters, 2008). All words, numbers and sentences were administered in sound-field through a single speaker (Klein + Hummel $O$ $110 \mathrm{D})$, positioned $1 \mathrm{~m}$ in front of the subject, connected to a laptop. The subjects used their own speech processor without a contralateral hearing aid. The APEX 3 test platform (Francart et al., 2008) was used to present the LIST and LINT stimuli. The listening effort test used the Oldenburg Measurement Applications software package, developed by Hörtech Oldenburg (www.hoertech.de). Stimuli for the spectral ripple discrimination test were created using MATLAB (The MathWorks, Inc.).
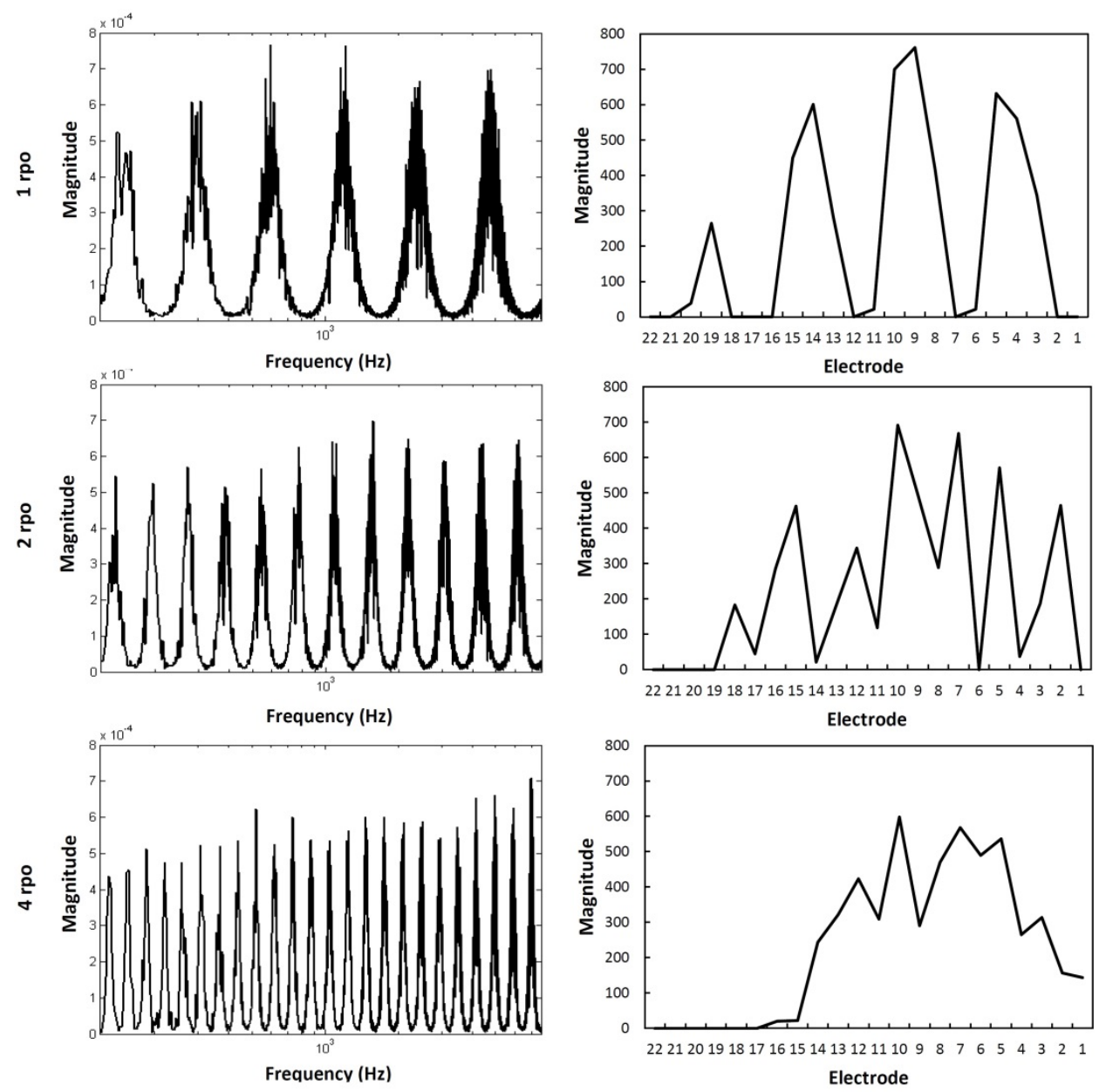

Figure 1. Spectra of the spectral ripple input stimulus (left) and output spectra of a $\mathrm{Cl}$ with 22 active electrodes (right), for ripple densities $1.000,2.000$ and 4.000 rpo. 
Chapter 3

\section{RESULTS}

\section{Electrode discrimination testing}

All 22 electrodes were active in the clinical map of five of the six subjects. For subject 1 (S1), six electrodes could not be used for testing due to impedance problems. Pulse widths of $25 \mu$ s were used, except for subject 5 (S5), where the pulse width was changed to $50 \mu \mathrm{s}$ due to out of compliance issues. The MCL of the central electrode, which was electrode number 12 for all six subjects, was on average 73\% (range 63-79\%) of the dynamic range between T- and UCL-levels. Good test-retest reliability was indicated by the intraclass correlation coefficients (ICC) for agreement, which ranged from .744 to .968 for the two runs of the electrode discrimination test. The range of the electrode discrimination difference limens (EDDLs), as well as the mean EDDL per subject, are presented in Table 2. Difference limens ranged from 0.5 to 7.125 over all subjects and reference electrodes. Subjects' average EDDLs ranged from 1.17 for the best (S2) to 3.88 for the worst performer (S5). For S1, the average value is preceded by a " $\leq$ " sign since there were a large number of deactivated electrodes contributing to an artificially increased average.

For each subject, the EDDLs are displayed in Figure 2. When the last four reversals are alternately on the reference and adjacent comparison electrode, the resulting difference limen is 0.5 (i.e., perfect discrimination). A striking similarity between the discrimination patterns of all subjects except S1, were smaller EEDLs for the apical and mid-electrodes, and larger EDDLs for more basally located electrodes. When dividing reference electrodes into apical (electrodes 16-22), middle (electrodes 8-15) and basal (electrodes 1-7) and calculating the average EEDL for S2 to S6, mean EEDLs were 1.16, 1.86 and 3.3 for the apical, middle and basal categories respectively. S1 was excluded due to the large number of missing values. A one-way repeated measures ANOVA indicated that that the mean difference limen was significantly affected by electrode category $(\mathrm{F}=18,84, \mathrm{df}=2,8, p=$ .001). Pairwise comparisons with a Bonferroni correction revealed significant differences between apical and basal ( $p=.034)$, and between middle and basal ( $p=.024)$, but not between apical and middle $(p=.149)$.

\section{Comparing the basic and experimental map}

The parameters of the basic map (BM) and the experimental map (EM) per subject can be found in Table 2. The number of deactivated electrodes based on the electrode discrimination results ranged from 4 (S1) to 14 (S5). The number of maxima was reduced for all subjects, with a reduction to four maxima for S5 and S6. For five out of six subjects the EM sounded softer than the BM; C-levels were therefore increased in live-mode by 3 to $7 \mathrm{CL}$ for these subjects.

\section{Speech understanding tests}

Individual subject and group median results for both the BM and the EM on the CNC monosyllabic word test are presented in Figure 3. Although median scores were slightly 


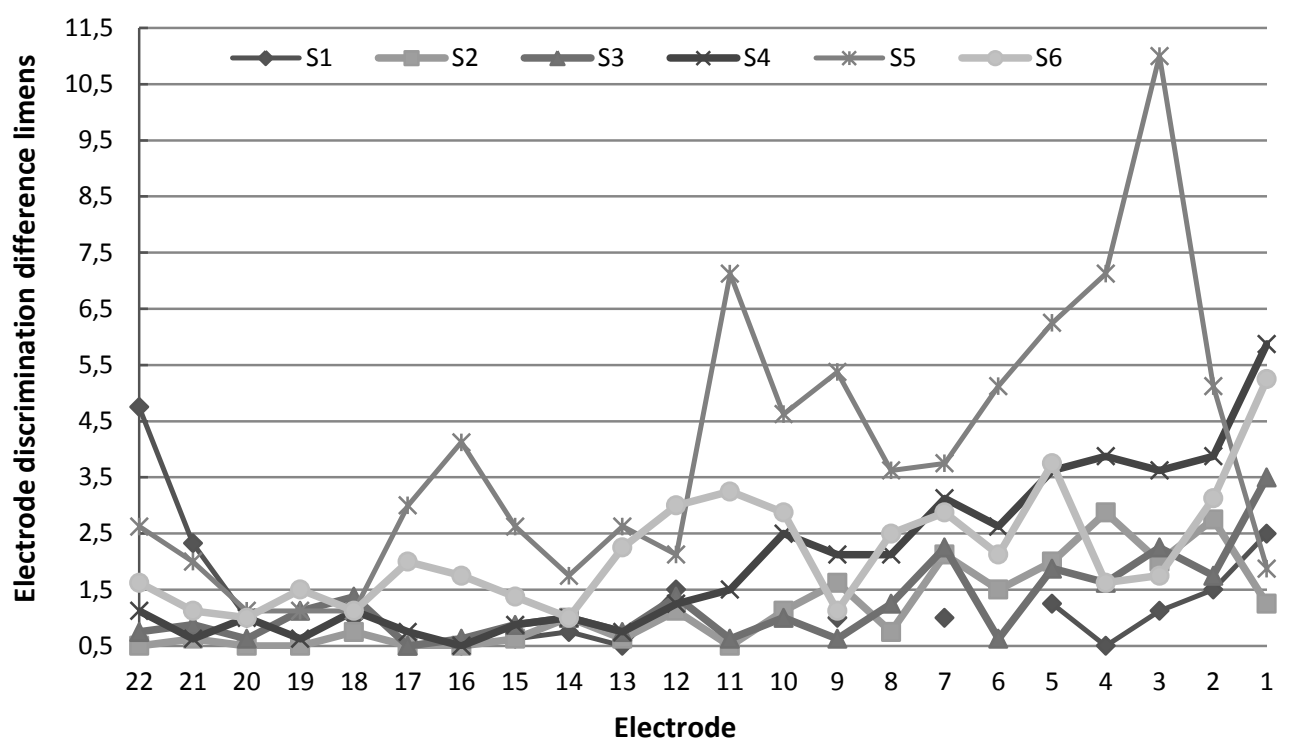

Figure 2. The electrode number of the reference electrode is represented on the $X$-axis; the electrode discrimination difference limen is displayed on the $Y$-axis. Reference electrode number 22 on the left is the most apical electrode; reference electrode number 1 is the most basal electrode. In case of perfect discrimination EDDLs would be 0.5 (lower boundary).

lower with the EM for the 75 and $65 \mathrm{~dB}$ administration levels, these differences were not statistically significant according to the Wilcoxon signed-rank test $(p=.893, p=.463$ and $p$ $=.074$ for the 55,65 and $75 \mathrm{~dB}$ administration levels, respectively).

The LIST was administered in quiet to all subjects except S5, for whom the test was too difficult. The LINT in quiet was also administered to S2, S4, S5 and S6. Subject mean and group median results for both maps are presented in Figure 4 . The Wilcoxon signed-rank test revealed no significant difference between the scores obtained with the BM or the EM for the LIST ( $p=.414$ ) or LINT ( $p=1.0)$ in quiet. In order to analyze possible differences between BM and EM on an individual subject level, the smallest detectable change (SDC) in test score was calculated ${ }^{6}$. It represents the minimal change in score for this change to be real, that is, not due to measurement error. Based on the results of the five subjects on the LIST in quiet, the SDC was $12.4 \%$. As can be seen in Table 3, only the difference score of S2 was statistically significantly larger than the SDC, indicating a lower recognition score when wearing the EM. On the LINT in quiet, none of the difference scores between BM and EM reached the SDC of $11 \%$ (see Table 3).

\footnotetext{
${ }^{6}$ The Smallest Detectable Change (SDC) is calculated as $1.96 * \sqrt{ } 2 * \mathrm{SEM}$. The standard error of measurement (SEM) is based on the square root of the error variance of the ICC model for consistency, and divided by three given that there are three repeated measures.
} 


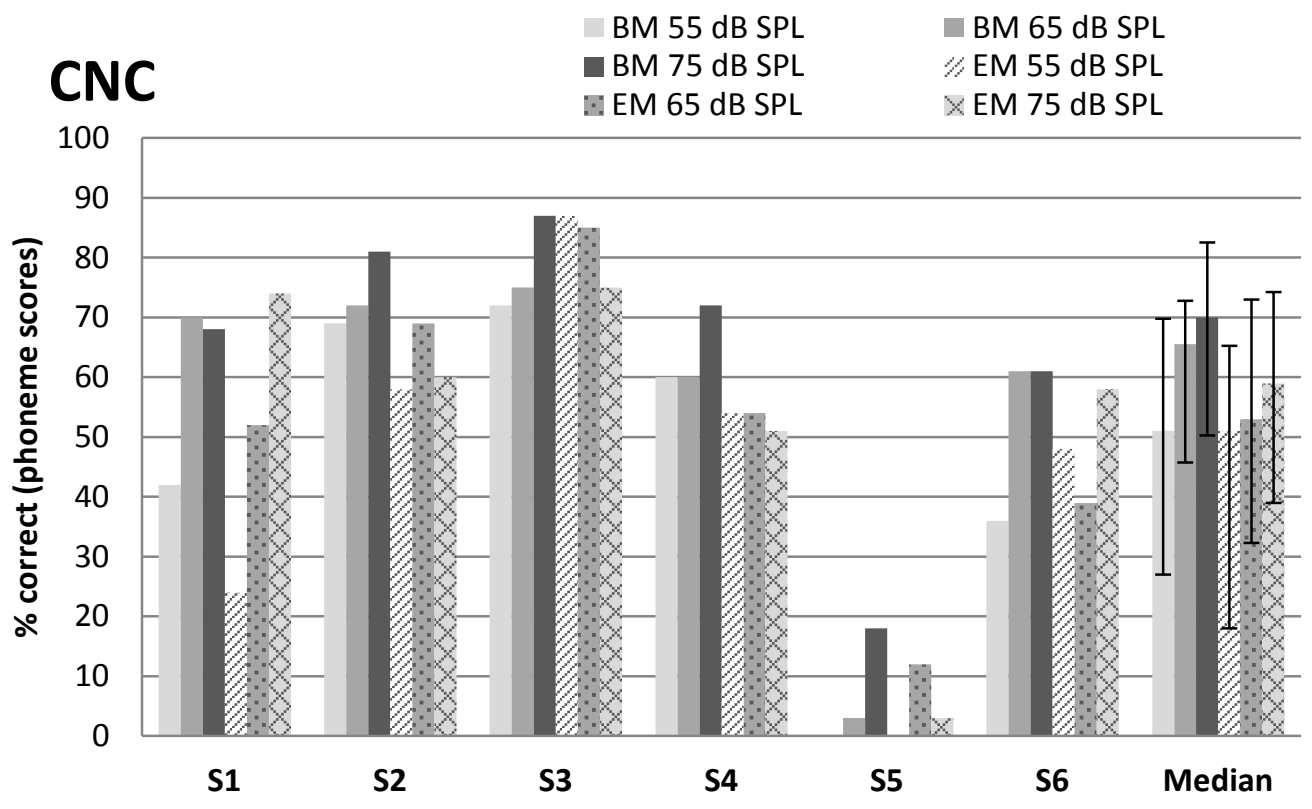

Figure 3. Individual and group median phoneme scores on the CNC test for the 6 subjects, with the BM and the EM, at 55, 65 and $75 \mathrm{~dB}$ SPL. Error bars for the median represent the interquartile range.

\section{LIST/LINT in quiet}

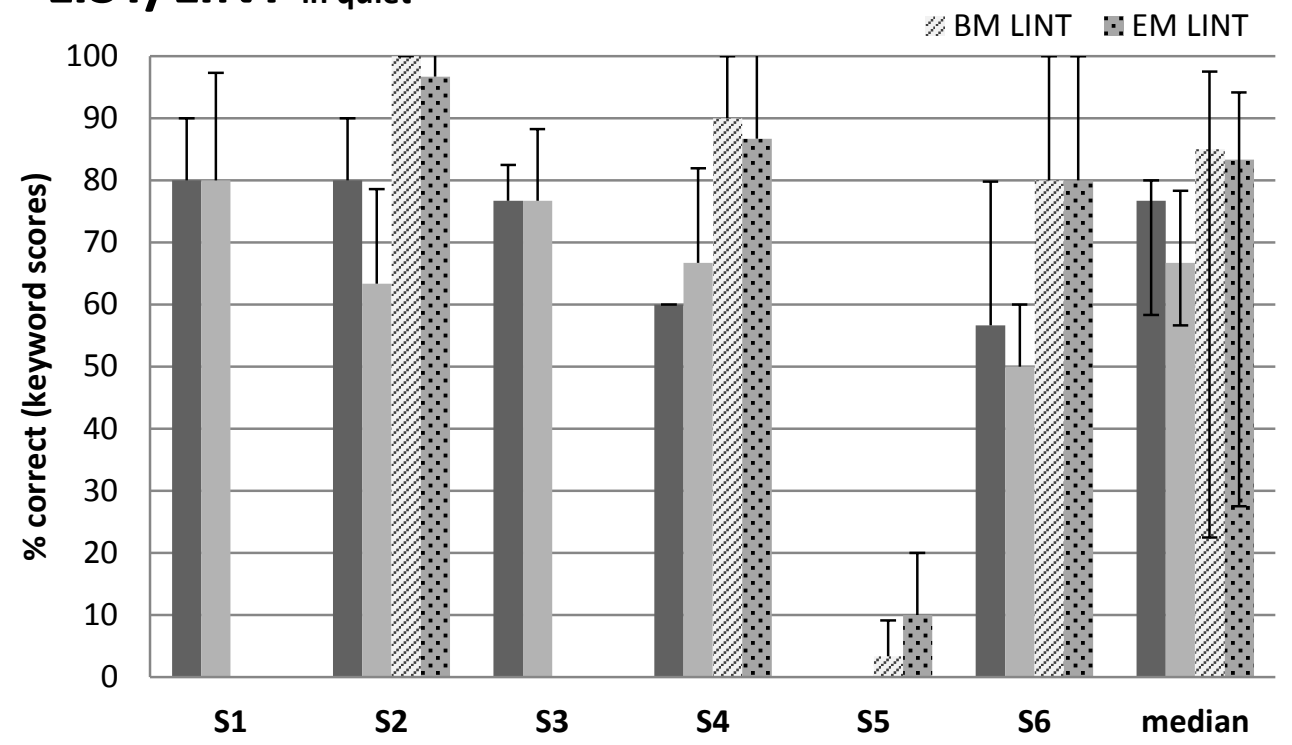

Figure 4. Subject mean and group median \% correct scores on the LIST and/or LINT in quiet for all subjects, with the $\mathrm{BM}$ and the EM. Error bars represent 1 SD for the subject means and the interquartile range for the group median scores. 
Table 3. Individual difference scores averaged over 3 measurements

\begin{tabular}{cccccc}
\hline Subject & $\begin{array}{c}\text { LIST silence } \\
(\%)\end{array}$ & $\begin{array}{c}\text { LINT silence } \\
(\%)\end{array}$ & $\begin{array}{c}\text { LIST noise } \\
\text { (dB SNR) }\end{array}$ & $\begin{array}{c}\text { LINT noise } \\
\text { (dB SNR) }\end{array}$ & $\begin{array}{c}\text { Spectral } \\
\text { ripple (rpo) }\end{array}$ \\
\hline S1 & 0 & NA & $1.75^{*}$ & NA & -0.09 \\
S2 & $-16.67^{*}$ & -3.33 & - & $4.42^{*}$ & $-0.65^{*}$ \\
S3 & 0 & NA & $1.78^{*}$ & NA & -0.19 \\
S4 & 6.66 & -3.33 & - & $-4.66^{*}$ & 0.05 \\
S5 & NA & 6.67 & NA & NA & -0.03 \\
S6 & -6.66 & 0 & - & 0.87 & $-0.31^{*}$ \\
\hline
\end{tabular}

A negative sign before the number indicates a decrease in test score from BM to EM (NA = test not administered, $-=$ no usable result,$^{*}=$ significant difference based on the SDC as calculated with the data from the subjects in this study)

On the LIST in noise, only two (S1 and S3) of the five tested subjects obtained a signal-tonoise ratio better than $10 \mathrm{~dB}$ SNR. The LINT in noise was therefore administered to S2, S4 and S6 instead. S5 was not able to perform a test in noise. Individual results with the BM and EM on either the LIST or LINT in noise can be seen in Figure 5. For S1 and S3, results were slightly better with the EM, with difference scores larger than the smallest detectable change of $1.17 \mathrm{~dB}$ SNR (Table 3). This is lower than the $1.8 \mathrm{~dB}$ SNR obtained for a group of 17 postlingual $\mathrm{Cl}$ users (unpublished data), indicating that the slightly better scores for S1 and S3 do not represent a clinically relevant improvement. On the LINT in noise, an improvement greater than $1.5 \mathrm{~dB}$ was obtained by S2 and S4, with S2 performing significantly better and S4 significantly worse for the EM. For S6 there was no significant difference. For the LIST in noise, calculation of the SDC was based on the results of just three subjects.

\section{Listening effort test}

The listening effort test was administered to all subjects except S5. There was no difference between the BM and the EM for either for the total listening effort averaged over the six signal-to-noise-ratios $(p=.68)$ or for any of the separate signal-to-noise-ratios, indicating no change in listening effort in noise.

\section{Spectral ripple discrimination test}

The spectral ripple test was administered to all six subjects. The individual discrimination thresholds ranged from 0.303 to 1.823 rpo for the BM and from 0.267 to 1.167 rpo for the EM as can be seen in Figure 6. The median threshold was 0.925 rpo with the BM and 0.855 rpo with the EM, this difference not being statistically significant (Wilcoxon signed-rank test, $p=.075$ ). On an individual subject level, the result with the EM was statistically significantly worse for both S2 and S6, for whom the SDC was 0.26 rpo (see Table 3). 
Chapter 3

\section{Questionnaire}

Questionnaire scores obtained for the BM and the EM did not differ ( $p=.225$, Wilcoxon signed-rank test). The EM was attributed a statistically significantly lower score for the Visual Analogue Scale ( $p=.042$, Wilcoxon signed-rank test). Three out of six subjects indicated "no preference" regarding the BM or EM programs, while two preferred the BM and one the EM.

\section{LIST/LINT in noise}

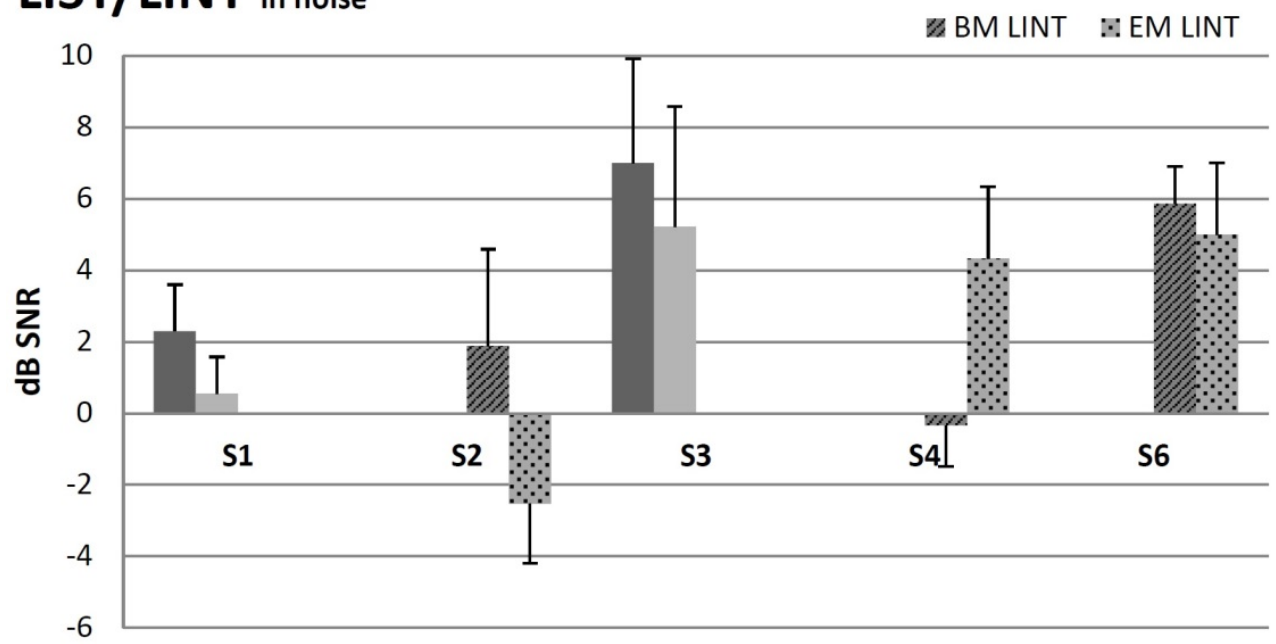

BM LIST $\quad$ EM LIST

Figure 5. Subject mean scores in dB SNR on the LIST or LINT in noise, for all subjects except S5. Error bars represent 1 SD.

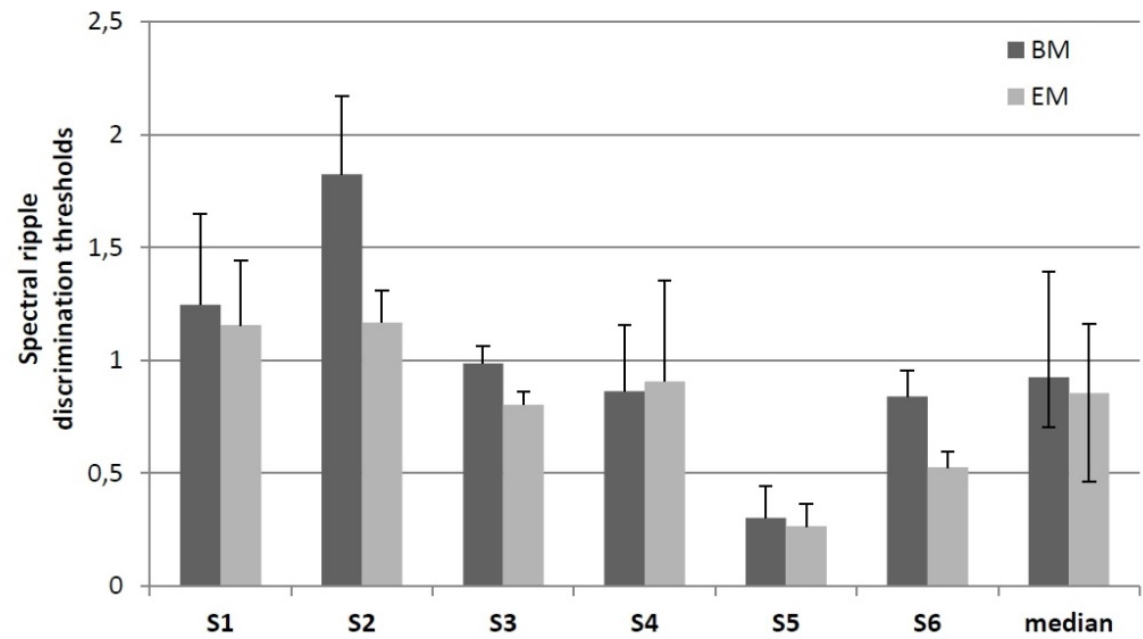

Figure 6. Subject mean and group median ripple discrimination thresholds for the BM and the EM. Error bars represent 1 SD for the subject mean and the interquartile range for the group median scores. 


\section{DISCUSSION}

The absolute electrode discrimination difference limens (EDDLs) found in this study concurred with those found in other studies with prelingually deafened subjects (Busby \& Clark, 1996, 2000). Although four out of our six subjects obtained a difference limen of 0.5 for at least one reference electrode, the observed range in difference limens (0.5 to 7.125) confirms that in many cases adjacent and even further separated electrodes cannot be discriminated by this group of $\mathrm{Cl}$ users. A limitation of the adaptive method used to determine the EDDL however, is that it assumes that discrimination improves when electrodes are further apart, which is not always necessarily the case, for instance, when there is a tip fold-over of the electrode array.

A striking observation in this study is the clearly poorer performance for the basal reference electrodes. This pattern was also found by Zwolan et al. (1997) in their three early deafened subjects, Henry et al. (2000) for postlingually deafened subjects, and in a pitch discrimination study by Kwon and van den Honert (2006). However, Busby and Clark (2000) did not observe a poorer performance basally, although electrode number 8 was the most basal reference electrode tested. A plausible explanation may be that most of our subjects did not like the sharp, often unpleasant sensation caused by stimulation of the basal electrodes, hampering discrimination tasks for these electrodes. Secondly, it may be that during the loudness balancing procedure, a drift occurred towards the basal electrodes. In this way, balanced levels could have become gradually softer, again due to the sharp sensation of stimulation on these electrodes, which in turn made discrimination more difficult. Thirdly, it is known that the amount of surviving spiral ganglion cells diminishes with a long period of profound deafness (Hardie \& Shepherd, 1999). As suggested by Pfingst et al. (2008), the number and condition of the activated neurons might be inferior at "ineffective" sites. It can be hypothesized that there are likely to be less surviving spiral ganglion cells in the basal region of the cochlea, where duration of deafness can be expected to be the longest for most subjects with prelingual extensive hearing loss.

For the sample as a whole, no significant differences were found between the two maps on either the CNC test or the LIST in silence. Sample comparison of the BM and EM could not be made for the speech tests in noise. At the individual subject level some significant differences were found between BM and EM, albeit not always in the same direction. Given that the SDC calculations were based on a small number of subjects, they were likely smaller than the true SDC's, as was illustrated for the LIST in noise. Hard conclusions can therefore not be drawn. This together with the fact that subjects did not experience a change in the amount of effort they needed to listen to and understand sentences or numbers in noise may be an indication that our findings are due to chance, and that there is no difference in speech understanding between the basic and the experimental map. 
The findings of the present study are not in agreement with those by both Zwolan et al. (1997), and more recently Saleh et al. (2013), who found significant improvements on tests of speech understanding, for at least some of their subjects, when the map contained only discriminable electrodes. There are a number of possible explanations for this lack of concordance.

First, the use of the MPEAK strategy by Zwolan et al. (1997) compromises comparison to results obtained with current speech processing strategies, like ACE. The MPEAK strategy specifically encodes F1 and F2 by stimulating individual electrodes; therefore it makes sense that by enlarging the contrast between the electrodes used for the representation of $F 1$ and F2, speech understanding scores improve.

Second, it might be that the reduction of the number of maxima in the present study had a negative influence on performance with the experimental map. Plant, Whitford, Psarros, and Vandali (2002) found that varying the number of maxima between 6 and 16 had little effect on speech understanding in silence, but that a 6-maxima program was significantly worse in noise. It has also been described by Qazi, van Dijk, Moonen, and Wouters (2013) that "wrong maxima selection" is likely to occur when listening to speech in noise with the ACE strategy, with a large portion of maxima being occupied by noise. The reduction in number of maxima could therefore have influenced our results in noise, but not in silence. It can be assumed that this factor did not have an effect on the results by Saleh et al. (2013), since they used a variety of speech processing strategies, and did not mention a reduction of maxima for the Nucleus ${ }^{\circledR}$ users.

Moreover, the prelingually deafened subjects in the present study might not have been completely adjusted to the altered frequency allocation consequent to the deactivation of electrodes. Zwolan et al. (1997) applied the default frequency allocation available in the software for both maps, but given the use of the MPEAK strategy this redistribution is likely to have had fewer consequences, and a positive effect of the experimental map could occur even without an adjustment period. In the more recent study by Saleh et al. (2013) it is not mentioned how the frequency allocation is changed, although it can be assumed that re-allocation was automatically implemented by the $\mathrm{Cl}$ software of the various brands. In their study, just as in the present one, subjects were given one month to adapt to the experimental program since it is known that changes in frequency allocation require an adjustment period. Fu, Shannon, and Galvin (2002) found that three months of experience with a map that had a severely shifted frequency allocation was not enough to attain the original level of speech understanding of three experienced $\mathrm{Cl}$ listeners. A model was developed based on these results, which suggested that these subjects were not able to completely alter their internal representations or "phoneme labels", in favor of the new situation (Sagi, Fu, Galvin, \& Svirsky, 2010). In the current study, some subjects explicitly reported that the EM required an adjustment with respect to how everything sounded. It is possible that prelingually deafened $\mathrm{Cl}$ users generally 

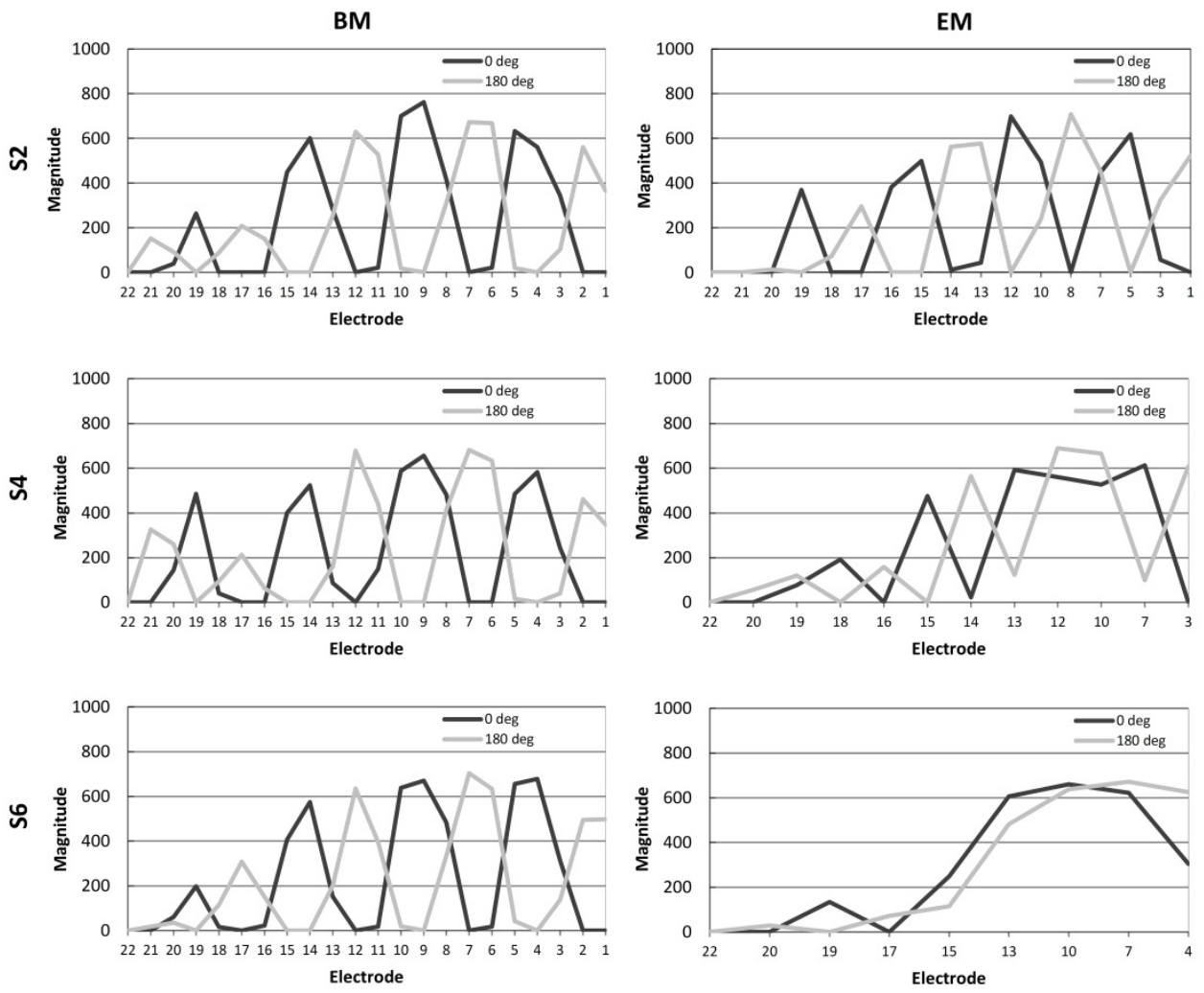

Figure 7. Output spectra for the 1 rpo spectral ripple stimuli obtained for the BM (left) and EM (right) of S2 (top), S4 (middle) and S6 (bottom).

require more time to form stable internal representations of sounds, especially given their limitations on a central auditory level, and that the one month adaptation period in this study was not sufficient to adjust to the albeit limited frequency shift.

The fourth factor that might have limited the potential improvement with the EM in this study, is related to the fact that deactivated electrodes were mainly located in the basal part of the array. This improved resolution mainly in the high-frequency region, whereas fine spectral resolution is more important for low to mid-frequencies (McKay, 2005). Also, Henry et al. (2000) only found a significant correlation between electrode discrimination abilities and speech information only up to $2680 \mathrm{~Hz}$. Details regarding the deactivation pattern were not provided by Saleh et al. (2013).

Spectral ripple discrimination thresholds also did not differ between the BM and the EM. At the individual subject level, S2 and S6 even performed significantly worse with the EM. Our hypothesis that a map consisting of discriminable channels leads to better performance on a test of spectral resolution was therefore not confirmed. We wondered whether this could be explained by the reduction in the number of electrodes. Shifted channels are broader in the EM than in the BM and contain a larger frequency range. 
When ripple density increases, there is an increased possibility that both a peak and a valley fall into the same frequency band, thus reducing the number of peaks and valleys and flattening the output spectrum. This was verified by measuring the electrode outputs of spectral ripple stimuli with different ripple densities presented in sound field to a Nucleus ${ }^{\circledR}$ sound processor that contained the exact maps of our subjects. A Nucleus ${ }^{\circledR}$ Freedom ${ }^{\mathrm{TM}}$ implant emulator was used for the recordings and data were analyzed with MATLAB (The MathWorks, Inc.). An illustration of the differences between the basic and experimental maps of S2, S4 and S6, who had a relatively decreasing number of active electrodes in their EM, can be seen in Figure 7 for the 1 rpo stimulus: the output spectrum is increasingly flattened with the EM for S2 to S6, when the number of active electrodes becomes smaller. The flattening generally occurred at lower ripple densities when the map contained less than 22 active electrodes. We obtained an estimation of the amount of available contrast for both BM and EM, per subject and per ripple density. We wanted to see if possible differences in the amount of contrast between the BM and EM, could be related to the subjects' actual ripple discrimination performance. We did this by calculating the difference between outputs of the phase-shifted stimuli and averaging them over the electrodes according to the method described by Croghan et al. (2013); see Figure 8. For S2 and S6 a significant difference in performance between the BM and EM was found on the ripple test. The majority of reversals in the adaptive procedure of S6 occurred at 0.707 rpo with the EM, while for the BM the stimulus with 0.707 rpo was mostly discriminated correctly. It can be seen in Figure 8 that the average difference between the output spectra for the phase-shifted stimuli was smaller for the EM, and declined more rapidly from 0.5 rpo onwards for the EM, whereas it remained constant for the BM. This may explain the worse performance by S6 for the EM. At the same time, S2 also performed worse for the EM, although there were no clear differences between BM and EM at 1.414 rpo, which is the density S2 could almost never discriminate in the adaptive procedure for the EM. No significant difference was found between ripple thresholds of S4, whereas a smaller contrast with the EM is clearly visible in Figure 8, starting from 0.707 rpo. For the remaining subjects S1, S3 and S5, the ripple densities where reversals occurred during the adaptive procedure were well below the ripple densities where the output spectrum became seriously disturbed. Therefore, we conclude that the amount of available spectral contrast available in the spectral ripple stimuli, and the reductions herein with the EM, could not be related to the ripple discrimination results.

Finally, the fact that there were no significant differences between BM and EM for the questionnaire concurs with the other results. For the Visual Analogue Scale, the EM rated statistically significantly lower than the BM which may be attributable to the required adjustment to the sound quality of the EM. Or it might be simply because our subjects did not feel as comfortable with the new map as with the old one, reflecting the adjustment to the internal representations of (speech) sounds. 


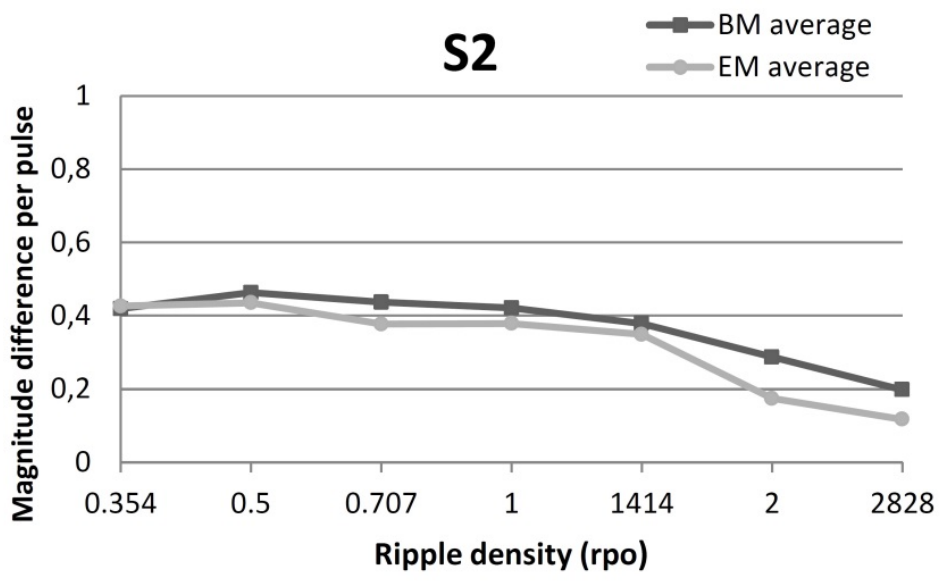

S4

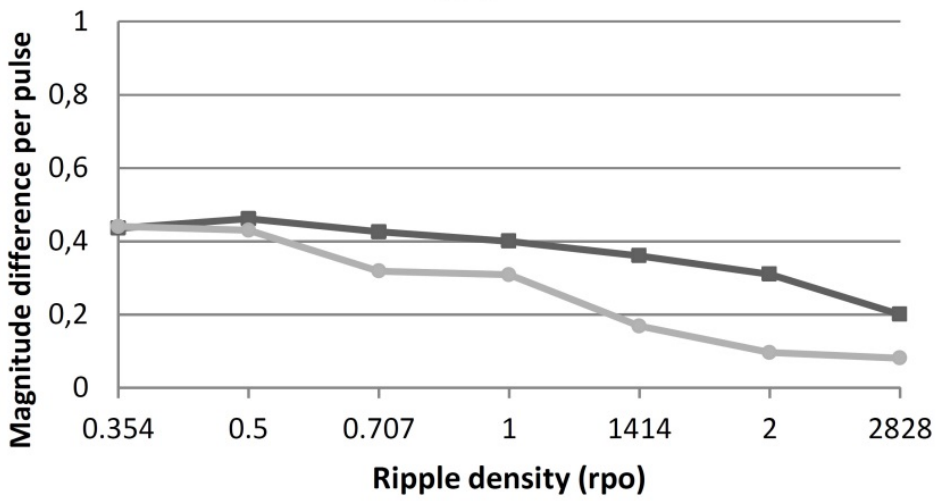

S6

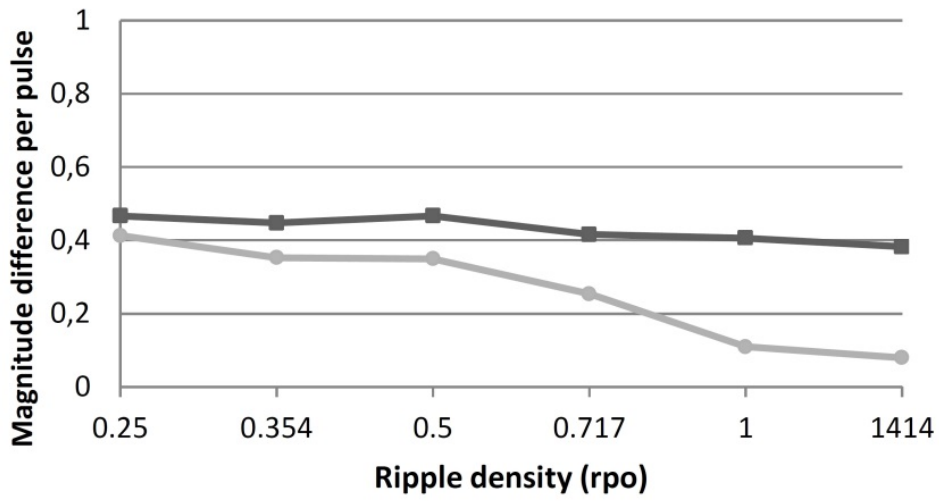

Figure 8. Average difference between output spectra of the phase-shifted stimuli of increasing ripple densities, ranging from 0.354 to 2.828 rpo for S2 and S4, and from 0.25 to 1.414 rpo for S6. The dark grey line represents the contrasts measured with the BM, the light grey line represents the contrasts measured with the EM. 


\section{CONCLUSIONS}

In this study, the electrode discrimination abilities of six prelingually deafened $\mathrm{Cl}$ users were investigated. The electrode discrimination difference limens ranged from 0.5 to 7.125 over all subjects and reference electrodes, confirming our first hypothesis that subjects were not able to discriminate between all adjacent electrodes. Across-site variations were observed, with significantly larger limens for basally located electrodes. Based on these findings, an experimental map containing only discriminable electrodes was devised to test our second hypothesis, which was whether such a map could improve auditory performance. However, this experimental map did not appear to be superior to the basic map for any of the subjects, on tests of speech understanding in quiet and in noise, spectral ripple discrimination or listening effort. Moreover, subjects generally preferred the basic to the experimental map. Our second hypothesis was therefore not confirmed. Factors that may have influenced the observed outcomes were (1) the reduction in the number of maxima in the experimental map, (2) the possibility that the adaptation period of four weeks was too short for these prelingually deafened subjects to become accustomed to the altered frequency allocation, and (3) the fact that a large proportion of the deactivated electrodes were located in the basal region of the cochlea, where fine spectral resolution is less important.

The current study was based on the results of only six subjects, hereby limiting generalizability. Besides testing more subjects, the following recommendations for future studies can be made: use electrode discrimination performance to adjust $\mathrm{Cl}$ fitting, avoid reducing the number of maxima (in case of the ACE strategy) and create a good balance of deactivated electrodes along the array. It may also be advisable to extend the adaptation period with the new map, or to perform the experiment in a cohort of recently implanted prelingually deafened adults, who have not yet become accustomed to a particular frequency allocation. 
Chapter 4

\section{Systematic review on late cochlear implantation in early deafened adults and adolescents. Part 1: clinical effectiveness}

Under review

Joke Debruyne

Miranda Janssen

Jan Brokx 


\section{ABSTRACT}

Objectives: Cochlear implantation in early-deafened patients, implanted as adolescents or adults, is not always advised due to poor expected outcomes. In order to judge whether such reluctance is justified, the current systematic review aimed to gather all available evidence on postoperative outcomes obtained by early-deafened patients using a stateof-the art cochlear implant (Cl).

Design: Five electronic databases (PubMed, Embase, the Cochrane library, CINAHL, and Psyclnfo) were systematically searched for studies in English, French, German or Dutch, published between 2000 and September 2017. Studies that reported pre- and postoperative outcomes on any measure of speech or sound perception, audiovisual or subjective benefit (quality of life) were included. Study quality was assessed with the Institute of Health Economics (IHE) quality appraisal tool for case series studies.

Results: The systematic search and subsequent full-text evaluation identified 38 studies meeting the inclusion criteria. Only a limited number of studies were judged to be of high quality according to the IHE tool, with lack of (clear) presentation of relevant study information being a recurring problem. Twenty-five studies presented viable outcomes on open-set speech understanding in quiet: mean postoperative scores obtained with $\mathrm{Cl}$ remained below $50 \%$ for the vast majority of studies; significant postoperative improvements were found in 16 studies, although this number might have been higher if adequate statistical testing had been performed in all studies. Eight studies observed increased audiovisual performance after implantation, which was statistically significant in 6 studies. A validated measure of hearing-related quality of life was used in only 5 of the 16 studies assessing subjective outcomes, showing significant postoperative improvements for most questionnaire domains. The relation between auditory and subjective outcomes was assessed in 2 studies, with contradictory results.

Conclusions: The current review showed that late cochlear implantation in early-deafened subjects resulted in significantly improved open-set speech perception, audiovisual speech perception, and (hearing-related) quality of life in the majority of the studies involved. Nonetheless, more and especially higher-quality research is needed in order to further increase our knowledge on the outcomes of cochlear implantation in this population. 


\section{INTRODUCTION}

The usefulness of cochlear implantation $(\mathrm{Cl})$ in postlingually deafened adults is well established. For one particular group of adult $\mathrm{Cl}$ candidates, uncertainties remain regarding the expected outcomes. These are the patients who have been profoundly deaf or severely hearing-impaired since birth or early childhood, and have a congenital, pre- or perilingual deafness. Early studies showed very poor auditory benefits of cochlear implantation in this population (Snik et al., 1997; Tong et al., 1988; Waltzman et al., 1992). A review in 2004 (Teoh et al., 2004a) showed that results obtained with the latest techniques had somewhat improved, but average outcome was still relatively poor, with most subjects implanted after the age of 12 obtaining only limited closed-set and minimal to no open-set speech understanding. These findings have had as a result that $\mathrm{Cl}$ clinics often opt not to implant early-deafened adolescents or adult $\mathrm{Cl}$-candidates, especially when the number of available Cl's is limited and choices need to be made with respect to who to implant. On the other hand, even early-day studies observed that despite limited outcomes, patient satisfaction within this specific group was relatively high (Hinderink et al., 1995; Zwolan et al., 1996). The hesitance to implant therefore has as a consequence that some patients who could be satisfied $\mathrm{Cl}$ users, are denied access to a cochlear implant. Most recent studies that focus on outcomes of cochlear implantation in this specific patient group still have relatively small sample sizes, likely due to the small number of implantations as compared to postlingually deafened adults and earlydeafened children. To know whether the conclusions of the latest review of 2004 (Teoh et al., 2004a) still stand, it would thus be interesting to look at the combined results of recent studies on cochlear implantation in this population.

In terms of outcomes, achieving a significant improvement in open-set, auditory-only speech understanding is often considered the main prerequisite of a successful cochlear implantation. In the postlingually deafened population we even see a shift towards speech understanding capabilities in noise. However, most early-deafened patients rely strongly on visual cues (i.e. lip reading) for their communication (Bernstein, Eberhardt, \& Auer, 2014). After receiving a cochlear implant, it is important that they are able to integrate the new sound with these visual cues, in order to enhance communication. The evaluation of the benefit of $\mathrm{Cl}$ in this population should therefore not be limited to auditory-only tests, since they are not a sufficient reflection of real-life, day-to-day communication, but should also include measures of audiovisual benefit.

It is widely acknowledged that the outcome of cochlear implantation should not purely be assessed with respect to various dimensions of speech understanding: cochlear implantation not only affects hearing abilities, but also has an impact on patients' healthrelated quality of life (QoL) (Klop et al., 2008; Loeffler et al., 2010). This is especially relevant for the population concerned, where gains in speech understanding are likely to 
be more limited than for adults with a postlingual onset of deafness. Early studies already suggested that patient satisfaction was high in the early-deaf population and not related to objective outcomes (Zwolan et al., 1996). This systematic review therefore also aimed to shed more light on non-auditory outcomes in this population, as well as on their relation with auditory outcomes.

In summary, this systematic review on cochlear implantation in early-deafened, but lateimplanted $\mathrm{Cl}$ users aimed to gather all available, relevant literature on cochlear implant outcomes of this specific patient group with respect to:
a. open- and closed-set speech understanding;
b. audiovisual benefit;
c. subjective benefit and health- or hearing-related quality of life.

\section{METHODS}

\section{Eligibility Criteria}

\section{Population}

For a study to be included, subjects had to be implanted unilaterally with a multichannel cochlear implant (any brand). At implantation, subjects had to be adolescent or adult, with a pre- or perilingual age at onset of deafness or severe hearing loss. Studies where (part of the) subjects were children, or adolescents/adults implanted in childhood, were not included in the current review since we wanted this review to contain homogeneous data on early-deafened adolescents or adults who received their cochlear implant well after the end of the language acquisition period. On the other hand, we did not impose a specific age limit either, since this could have led to the exclusion of studies containing a small minority number of subjects implanted at a slightly younger age. Stricter age limits are however incorporated in the IHE (Institute of Health Economics, Alberta, Canada) study quality assessment (see item 7 in Table 1), in order to judge the homogeneity of the study sample. If a study encompassed data from both children and adults, or from both pre- and postlingually deafened subjects, the study was included only if the data from the group that met the inclusion criteria were separately available and separately analyzed.

\section{Outcomes}

Studies had to contain one of the following outcome measures: a measure of speech or sound perception, a measure of lipreading, or a quality of life assessment/subjective benefit questionnaire (either hearing- or health-related). The results had to be analyzed, at least descriptively, on a group level. Outcomes needed to be assessed pre- and postimplantation for all subjects, or retrospectively (for certain quality of life/ subjective benefit measures). Alternatively, the outcomes had to assess audiovisual benefit.

\section{Study type, language and publication date}

Studies could be of any design, with the exception of case studies or very small samples $(n<5)$. Conference abstracts, theses, book chapters and letters were included if they met 
the other inclusion criteria. Study language could be English, German, French or Dutch. Since substantial changes in cochlear implant technology occurred between 1990 and 2000, publications of the year 2000 or later were included.

\section{Search Strategy}

A systematic literature search was performed on September 6th, 2017 in the following databases: PubMed, Embase, the Cochrane library, CINAHL, and Psyclnfo. In the search syntax (details in Appendix A), Medical Subject Heading (MeSH) terms (PubMed, the Cochrane library) or Thesaurus terms (Embase, CINAHL, Psyclnfo) were combined with Free-text terms. In order not to exclude very recent PubMed articles for which MeSH terms had not yet been attributed, an additional search was performed in PubMed without age-related search terms. Endnote was used to identify those studies without MeSH terms, which were then added to the group of studies as found through the standard search. A search through the reference lists of all included articles did not yield any additional relevant articles.

\section{Study Selection}

The guidelines in CRD's (Centre for Reviews and Dissemination) guidance for undertaking reviews in healthcare (Systematic reviews, crd's guidance for undertaking reviews in health care 2009) were used for conducting this review. As a first step, articles were screened on title and abstract by 2 independent reviewers, discarding irrelevant articles and studies that clearly did not meet the inclusion criteria. Differences in the selection of articles were resolved by consensus, following discussion. In the second phase, the retrieved full-text versions of the remaining articles were further compared with the inclusion criteria. Discrepancies between the 2 reviewers were again resolved by consensus, after discussion.

\section{Data Extraction}

For each study, the general characteristics of the included study group were identified, i.e. the number of included subjects, the age range at implantation and the communication mode of the subjects. Data on the relevant outcome measures were extracted, including the exact type of the outcome measure(s), outcome assessment times, test condition(s) and results (see Appendix B for a summary). When available, the outcomes of statistical analysis comparing assessment times, mainly preoperative vs. postoperative, were inventoried. Alternatively, main outcomes were summarized descriptively.

\section{Evaluation of Study Quality}

A tailored version of the Institute of Health Economics (IHE) quality appraisal tool for case series studies (Guo, Moga, Harstall, \& Schopflocher, 2016; Moga, Guo, Schopflocher, \& Harstall, 2012) was chosen as the quality assessment instrument. This tool is specifically developed for case-series studies, i.e. a single group of patients that receive an 
intervention and is followed retro- or prospectively, without comparison to a control group. The majority of studies included in this review had a case-series design, with cochlear implantation being the intervention. The IHE's checklist was developed by means of a modified Delphi process and contains 20 criteria in 8 categories in its original form. The guidance for rating each criterion was, as advised by the developers, adapted to fit the content of this review and can be found in Appendix C. Two of the original criteria, concerning potential co-interventions and blinded assessment of outcomes were omitted because they were not applicable to this type of intervention, resulting in a 18-criterion checklist. Quality assessment was again performed by two independent reviewers; discrepancies were resolved by consensus after discussion.

\section{RESULTS AND DISCUSSION}

\section{Search and Study Selection}

A total of 2385 manuscripts resulted from the 5 databases. After deduplication, the number was reduced to 1449 articles. Screening of title/abstract left 186 articles eligible for full-text review. Of these, 38 studies met the inclusion criteria and were included in the review. The PRISMA chart in Figure 1 contains an overview of the selection process, including more details on the reasons for exclusion.

\section{Quality Assessment: IHE's quality appraisal tool for case series studies}

An overview of the results from the quality assessment with the IHE's checklist is presented in Table 1; more details on the judgment per item can be found in Appendix D. As can be seen in Table 1, a number of quality items were met in almost every study: a clearly stated study aim (item1), outcome measures established a priori (item 9) and measured pre- and postoperatively (item 11), and patient follow-up at least 6 months (item 13). In more than half of the studies all relevant patient characteristics of the included study group were described (item $\mathbf{5}$ ), all subjects met the criteria regarding age at onset and age at implantation (item 7), the methods used for outcome measurement were appropriate (item 10), losses to follow-up were reported (item 14) and estimates of random variability were available or could be calculated from the raw data (item 15). Patient recruitment (item 4) took place consecutively in 16 of the 38 studies but was generally unclear (19 studies). Statistical testing was judged to be appropriate in only 16 studies, and inappropriate in 10 studies (item 12). In 2 studies the answer was "not applicable" due to the lack of statistical comparison of pre- and postop scores. On item 17, 6 studies that were mainly prognostic in nature did not have a specific conclusion statement with respect to the interventional part of the study and were attributed NA; of the remaining studies, the conclusion statement was supported by the results for 17 of them. In contrast, less than one third of the studies clearly stated both in- 


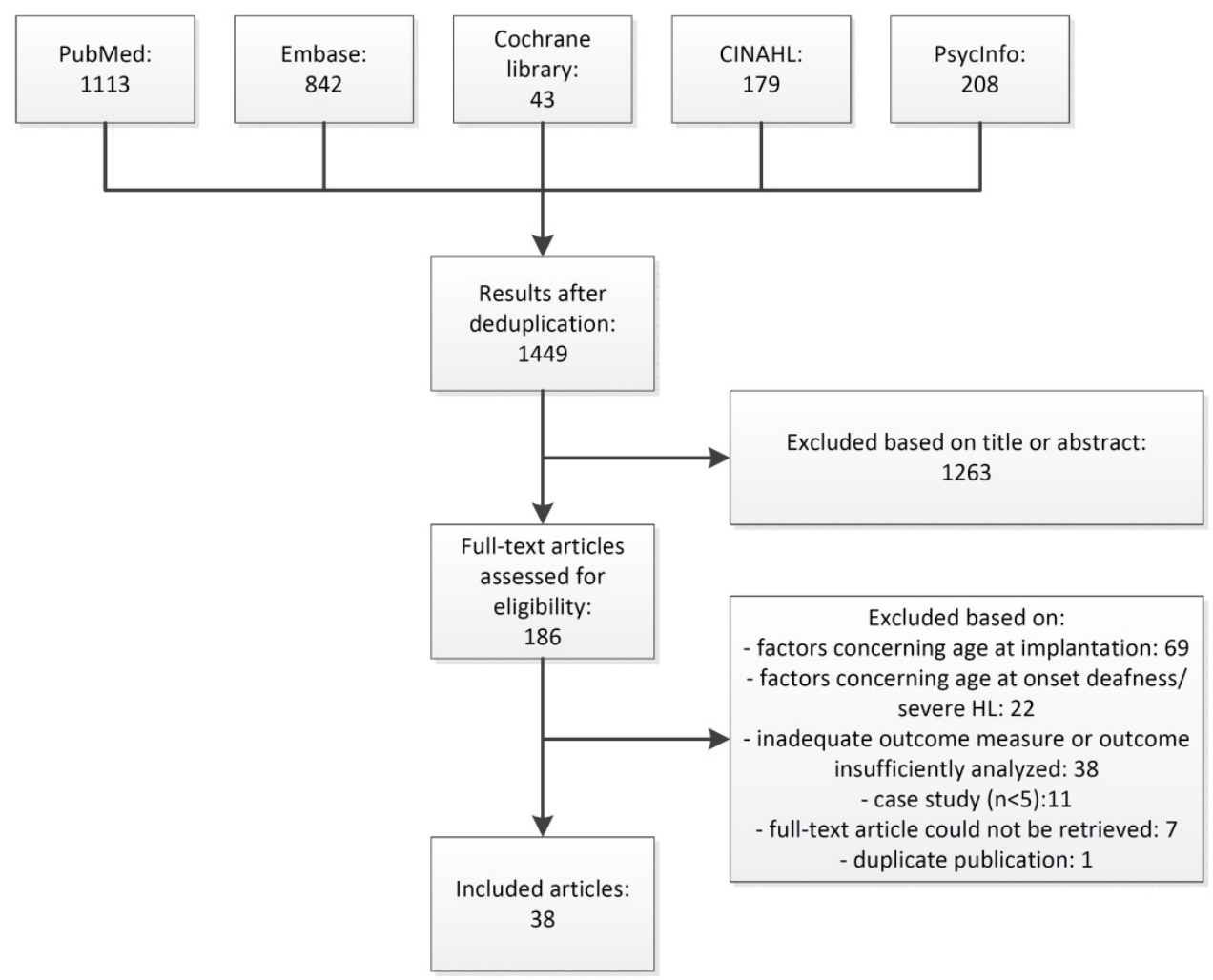

Figure 1. PRISMA chart of the study selection process.

and exclusion criteria (item 6) or contained a declaration concerning both competing interests and sources of support (item 18). Also, the vast majority of studies did not have a prospective design (item 2), did not describe all relevant items concerning the cochlear implantation (item 8), did not report on adverse events (item 16), and none were multicenter studies (item $\mathbf{3}$ ).

The number of quality items met per study ranged between 3 and 14 (final column of Table 1). In reality, some items are of course more important than others and failing important criteria may lead to the exclusion of a study for a certain analysis (e.g. item 12), whereas failing certain other criteria will have far less consequences (e.g. item 18).

\section{Outcomes}

A summary of all 38 included studies, providing general information on the main subject characteristics, outcome measures and reported results, can be found in Appendix B. As it was one of the inclusion criteria for the review, all 38 included studies contain a postoperative outcome measure related to either speech, sound perception or subjective outcomes. Results are presented, discussed and related to the outcomes of the IHE quality 
Chapter 4

Table 1. Overview of IHE quality assessment per study

\begin{tabular}{|c|c|c|c|c|c|c|c|c|c|c|}
\hline & 1. & 2. & 3. & 4. & 5. & 6. & 7. & 8. & 9. & 10. \\
\hline Arisi et al. 2010 & $\bullet$ & $\bullet$ & 0 & $?$ & $\bullet$ & $\bullet$ & $\bullet$ & $\bullet$ & $\bullet$ & 0 \\
\hline Bassim et al. 2005 & $\bullet$ & ० & ० & $?$ & \pm & \pm & ० & \pm & $\bullet$ & $\bullet$ \\
\hline Bellelli et al. 2016 & $\bullet$ & $?$ & ० & $\bullet$ & \pm & ० & $?$ & ० & $\bullet$ & $\bullet$ \\
\hline Bosco et al. 2013 & $\bullet$ & $?$ & ० & $?$ & $\bullet$ & $\bullet$ & $\bullet$ & \pm & $\bullet$ & \pm \\
\hline Caposecco et al. 2012 & $\bullet$ & 0 & ० & $\bullet$ & $\bullet$ & \pm & ० & \pm & $\bullet$ & \pm \\
\hline Chee et al. 2004 & $\bullet$ & $?$ & ० & $\bullet$ & \pm & ० & o & ० & $\bullet$ & o \\
\hline Craddock et al. 2016 & \pm & ० & ० & $\bullet$ & $\bullet$ & ० & o & \pm & $\bullet$ & $\bullet$ \\
\hline Cusumao et al. 2017 & $\bullet$ & o & o & $\bullet$ & \pm & $\bullet$ & $\bullet$ & o & $\bullet$ & $\bullet$ \\
\hline Derinsu et al. 2002 & $\bullet$ & $?$ & ० & ? & $\bullet$ & ० & $\bullet$ & \pm & $\bullet$ & o \\
\hline Duchesne et al. 2017 & $\bullet$ & $?$ & $?$ & $?$ & \pm & \pm & $\bullet$ & \pm & $\bullet$ & \pm \\
\hline Heywood et al. 2016 & $\bullet$ & o & ० & ? & $\bullet$ & \pm & ० & o & $\bullet$ & \pm \\
\hline Kaplan et al. 2003 & $\bullet$ & ० & o & $\bullet$ & $\bullet$ & \pm & o & \pm & $\bullet$ & \pm \\
\hline Klop et al. 2007 & $\bullet$ & $?$ & o & $?$ & $\bullet$ & $\bullet$ & $\bullet$ & \pm & $\bullet$ & $\bullet$ \\
\hline Kos et al. 2009 & $\bullet$ & $?$ & ० & $?$ & $\bullet$ & o & ० & \pm & $\bullet$ & $\bullet$ \\
\hline Kraaijenga et al. 2015 & $\bullet$ & ० & ० & $\bullet$ & \pm & \pm & $\bullet$ & ० & $\bullet$ & $\bullet$ \\
\hline Kumar et al. 2016 & $\bullet$ & O & ० & $?$ & O & \pm & $\bullet$ & O & $\bullet$ & \pm \\
\hline Mantokoudis et al. 2011 & $\bullet$ & ० & ० & ? & $\bullet$ & \pm & o & \pm & $\bullet$ & o \\
\hline Millette et al. 2011 & $\bullet$ & ० & ० & ○ & \pm & \pm & $\bullet$ & $\bullet$ & $\bullet$ & \pm \\
\hline Moody-Antonio et al. 2005 & $\bullet$ & $?$ & o & $\bullet$ & \pm & \pm & $\bullet$ & \pm & $\bullet$ & $\bullet$ \\
\hline Most et al. 2009 & $\bullet$ & $?$ & $?$ & $?$ & $\bullet$ & ○ & o & \pm & $\bullet$ & $\bullet$ \\
\hline O'Gara et al. 2016 & $\bullet$ & o & ० & $\bullet$ & \pm & $\bullet$ & $\bullet$ & o & $\bullet$ & $\bullet$ \\
\hline Peasgood et al. 2003 & $\bullet$ & $?$ & $?$ & $\bullet$ & $\bullet$ & ० & o & \pm & $\bullet$ & $\bullet$ \\
\hline Rousset et al. 2016 & $\bullet$ & $?$ & o & $\bullet$ & \pm & ० & $?$ & \pm & $\bullet$ & $\bullet$ \\
\hline Santarelli et al. 2008 & $\bullet$ & $?$ & o & $\bullet$ & $\bullet$ & $\bullet$ & $\bullet$ & $\bullet$ & $\bullet$ & $\bullet$ \\
\hline Schramm et al. 2002 & $\bullet$ & ० & ० & $\bullet$ & $\bullet$ & \pm & $\bullet$ & \pm & $\bullet$ & $\bullet$ \\
\hline Shin et al. 2015 & $\bullet$ & $\bullet$ & o & $?$ & o & \pm & $?$ & ० & $\bullet$ & $\bullet$ \\
\hline Shpak et al. 2009 & $\bullet$ & $?$ & ० & $?$ & $\bullet$ & ० & o & \pm & $\bullet$ & $\bullet$ \\
\hline Souza et al. 2011 & $\bullet$ & $\bullet$ & o & $?$ & \pm & \pm & $?$ & \pm & $\bullet$ & $\bullet$ \\
\hline Straatman et al. 2014 & $\bullet$ & $\bullet$ & ० & $\bullet$ & $\bullet$ & \pm & $\bullet$ & o & $\bullet$ & $\bullet$ \\
\hline Teoh et al. 2004 & $\bullet$ & $?$ & $?$ & $?$ & ० & \pm & $\bullet$ & \pm & ० & $\bullet$ \\
\hline Van der Marel et al. 2015 & $\bullet$ & $?$ & ० & $?$ & \pm & $\bullet$ & $\bullet$ & \pm & $\bullet$ & \pm \\
\hline van Dijkhuizen et al. 2011 & $\bullet$ & $\bullet$ & ० & ० & $\bullet$ & $\bullet$ & $\bullet$ & $\bullet$ & $\bullet$ & $\bullet$ \\
\hline van Dijkhuizen et al. 2016 & $\bullet$ & $\bullet$ & o & ० & $\bullet$ & $\bullet$ & $\bullet$ & \pm & $\bullet$ & $\bullet$ \\
\hline Waltzman et al. 2002 & $\bullet$ & $\bullet$ & $?$ & $?$ & $\bullet$ & o & $\bullet$ & \pm & $\bullet$ & $\bullet$ \\
\hline Watson et al. 2003 & \pm & ? & ? & ? & \pm & ० & $\bullet$ & o & $\bullet$ & \pm \\
\hline Yang et al. 2011 & \pm & $?$ & ० & $?$ & \pm & \pm & $\bullet$ & \pm & $\bullet$ & o \\
\hline Yucel \& Sennaroglu 2007 & $\bullet$ & $?$ & o & $\bullet$ & \pm & ० & ० & \pm & $\bullet$ & o \\
\hline Zeitler et al. 2012 & \pm & ० & ० & $\bullet$ & \pm & $\bullet$ & $\bullet$ & ० & $\bullet$ & \pm \\
\hline
\end{tabular}

$\bullet=$ Yes, $0=$ No, $\pm=$ Partial, ? = Unclear; NA = Not Applicable

IHE item overview: 1: Objective clearly stated?; 2: Prospective study?; 3: Multicentre study?; 4: Patients recruited consecutively?; 5: Relevant patient characteristics described (age at onset, age at implantation, communication mode)?; 6: Eligibility criteria clearly stated?; 7: Study entry at similar point in disease (onset $<4$ yrs and implantation $>10 \mathrm{yrs}$ ) ?; 8: Intervention clearly described (implant, coding strategy, rehabilitation); 9: Outcome measures established a priori?; 10: Outcomes measured with appropriate methods? 
Table 1. Continued

\begin{tabular}{|c|c|c|c|c|c|c|c|c|c|}
\hline & 11. & 12. & 13. & 14. & 15. & 16. & 17. & 18. & \# of Yes \\
\hline Arisi et al. 2010 & $\bullet$ & $\bullet$ & $\bullet$ & $\bullet$ & $\bullet$ & $\bullet$ & 0 & $\bullet$ & $14 / 18$ \\
\hline Bassim et al. 2005 & \pm & ○ & $?$ & $?$ & o & \pm & o & \pm & $3 / 18$ \\
\hline Bellelli et al. 2016 & $\bullet$ & $\bullet$ & $?$ & $?$ & $\bullet$ & $\circ$ & $\bullet$ & ○ & $8 / 18$ \\
\hline Bosco et al. 2013 & $\bullet$ & \pm & $\bullet$ & $?$ & $\bullet$ & o & $?$ & o & $9 / 18$ \\
\hline Caposecco et al. 2012 & $\bullet$ & $\bullet$ & $\bullet$ & $\bullet$ & $\bullet$ & $\bullet$ & $\bullet$ & $\circ$ & $11 / 18$ \\
\hline Chee et al. 2004 & $\bullet$ & $\bullet$ & ० & $\bullet$ & $\bullet$ & ○ & $?$ & ○ & $7 / 18$ \\
\hline Craddock et al. 2016 & $\bullet$ & o & $\bullet$ & $?$ & $\bullet$ & \pm & $\bullet$ & $\bullet$ & $9 / 18$ \\
\hline Cusumao et al. 2017 & $\bullet$ & $?$ & $\bullet$ & $\bullet$ & \pm & \pm & ? & \pm & $9 / 18$ \\
\hline Derinsu et al. 2002 & $\bullet$ & ० & $\bullet$ & $\bullet$ & $\bullet$ & ० & ० & ० & $8 / 18$ \\
\hline Duchesne et al. 2017 & $\bullet$ & $\bullet$ & $\bullet$ & ? & $\bullet$ & $\bullet$ & $\bullet$ & $\bullet$ & $10 / 18$ \\
\hline Heywood et al. 2016 & $\bullet$ & $\bullet$ & $\bullet$ & $\bullet$ & $\bullet$ & $\circ$ & $\bullet$ & $\bullet$ & $10 / 18$ \\
\hline Kaplan et al. 2003 & \pm & $?$ & $\bullet$ & $\bullet$ & $\circ$ & \pm & $\bullet$ & ० & $7 / 18$ \\
\hline Klop et al. 2007 & $\bullet$ & $\circ$ & $\bullet$ & $\bullet$ & \pm & \pm & $\bullet$ & \pm & $10 / 18$ \\
\hline Kos et al. 2009 & $\bullet$ & $?$ & $\bullet$ & ○ & $\bullet$ & \pm & $\bullet$ & ○ & $8 / 18$ \\
\hline Kraaijenga et al. 2015 & $\bullet$ & NA & $\bullet$ & $\circ$ & $\bullet$ & ० & NA & $\bullet$ & $8 / 16$ \\
\hline Kumar et al. 2016 & $\bullet$ & $?$ & ? & $\circ$ & \pm & ○ & $\circ$ & $\bullet$ & $5 / 18$ \\
\hline Mantokoudis et al. 2011 & $\bullet$ & $\bullet$ & $\bullet$ & $\bullet$ & ○ & $\circ$ & $\bullet$ & \pm & $8 / 18$ \\
\hline Millette et al. 2011 & \pm & ० & $\bullet$ & $?$ & $\bullet$ & ० & $?$ & ० & $6 / 18$ \\
\hline Moody-Antonio et al. 2005 & ० & $\bullet$ & $\bullet$ & ? & $\bullet$ & ○ & $\bullet$ & $\bullet$ & $10 / 18$ \\
\hline Most et al. 2009 & $\circ$ & $\bullet$ & $\bullet$ & $\bullet$ & $\bullet$ & $\circ$ & $\bullet$ & ० & $9 / 18$ \\
\hline O’Gara et al. 2016 & $\bullet$ & $?$ & $\bullet$ & $\bullet$ & $\bullet$ & \pm & NA & \pm & $10 / 17$ \\
\hline Peasgood et al. 2003 & $\bullet$ & $\bullet$ & $\bullet$ & $\bullet$ & $\bullet$ & \pm & $\bullet$ & ० & $11 / 18$ \\
\hline Rousset et al. 2016 & $\bullet$ & $\bullet$ & $\circ$ & $\bullet$ & $\bullet$ & $\circ$ & $\bullet$ & \pm & $9 / 18$ \\
\hline Santarelli et al. 2008 & $\bullet$ & \pm & $\bullet$ & $\bullet$ & $\bullet$ & ० & ० & $\circ$ & $13 / 18$ \\
\hline Schramm et al. 2002 & $\bullet$ & $\circ$ & $\bullet$ & $\bullet$ & $\bullet$ & $\bullet$ & ० & ○ & $11 / 18$ \\
\hline Shin et al. 2015 & $\bullet$ & $\circ$ & $\bullet$ & $\circ$ & ० & $\circ$ & $\circ$ & $\bullet$ & $7 / 18$ \\
\hline Shpak et al. 2009 & $\bullet$ & $\bullet$ & $\bullet$ & $\bullet$ & $\bullet$ & $\circ$ & $\bullet$ & \pm & $10 / 18$ \\
\hline Souza et al. 2011 & $\bullet$ & $\circ$ & $\bullet$ & $\bullet$ & $\bullet$ & $\circ$ & ० & ० & $8 / 18$ \\
\hline Straatman et al. 2014 & $\bullet$ & $\bullet$ & $\bullet$ & $\bullet$ & \pm & $\circ$ & $\bullet$ & \pm & $12 / 18$ \\
\hline Teoh et al. 2004 & $\bullet$ & $\circ$ & $\bullet$ & $?$ & $\bullet$ & ० & $?$ & \pm & $6 / 18$ \\
\hline Van der Marel et al. 2015 & $\bullet$ & NA & $\bullet$ & $\bullet$ & $\bullet$ & \pm & NA & $\bullet$ & $9 / 16$ \\
\hline van Dijkhuizen et al. 2011 & $\bullet$ & $\circ$ & $\bullet$ & $\bullet$ & $\bullet$ & $\bullet$ & NA & \pm & $13 / 17$ \\
\hline van Dijkhuizen et al. 2016 & $\bullet$ & $\bullet$ & $\bullet$ & $\bullet$ & $\bullet$ & $\bullet$ & NA & $\bullet$ & $14 / 17$ \\
\hline Waltzman et al. 2002 & $\bullet$ & $\bullet$ & $\bullet$ & $\bullet$ & $\bullet$ & \pm & $\circ$ & \pm & $11 / 18$ \\
\hline Watson et al. 2003 & $\bullet$ & ? & $\bullet$ & $\bullet$ & ० & $\circ$ & $\bullet$ & ० & $6 / 18$ \\
\hline Yang et al. 2011 & $\bullet$ & $?$ & $\bullet$ & $?$ & \pm & $\circ$ & NA & \pm & $4 / 17$ \\
\hline Yucel \& Sennaroglu 2007 & $\bullet$ & $\circ$ & $\bullet$ & $\bullet$ & $\bullet$ & $\circ$ & $\circ$ & ० & $7 / 18$ \\
\hline Zeitler et al. 2012 & $\bullet$ & $\bullet$ & $\bullet$ & $\bullet$ & $\bullet$ & $\circ$ & $\bullet$ & \pm & $10 / 18$ \\
\hline
\end{tabular}

$\bullet=$ Yes, $\mathrm{O}=$ No, \pm = Partial, ? = Unclear; NA = Not Applicable

IHE item overview: 11: Outcomes measured before and after the intervention?; 12: Statistical tests appropriate?; 13: Follow-up at least 6 months post implantation?; 14: Losses to follow-up reported?; 15: Estimates of random variability?; 16: Adverse events reported?; 17: Conclusions supported by results?; 18: Competing interests and sources of support reported? 
assessment per type of outcome measure, starting with outcomes on open- and closedset speech understanding tests, including tests in quiet and in noise (a), followed by outcomes of tests assessing audiovisual benefit (b), and finally outcomes related to subjective benefit and quality of life (c).

\section{a. Open- and closed-set speech understanding}

In 33 of the 38 included studies, performance before and after cochlear implantation was assessed using open- or closed-set set speech understanding, in an auditory-only test condition, generally in quiet but for a limited number of studies also in noise. For 6 studies, (part of) the results could not be used for further comparison ${ }^{7}$ due to missing data, lack of outcome analysis or unclear test conditions. Table 2 contains the summarized results of the remaining 27 studies: the number of subjects in these studies ranged from 8 to 77 and the age at implantation ranged from 8 to 74 years (not given in 4 studies). Table 2 further contains the communication mode of the included subjects, the outcome assessment times, the pre- and postoperative test condition and whether or not the difference between the pre- and postoperative score was significant.

Of the 27 studies in Table 2, 25 present results on one or more open-set speech recognition tests in quiet. Twelve studies measured open-set speech understanding by means of one-syllable CVC/CNC word tests in quiet (Cusumano et al., 2017; Klop, Briaire, Stiggelbout, \& Frijns, 2007; Kraaijenga et al., 2016; Rousset, Dowell, \& Leigh, 2016; Shpak, Koren, Tzach, Most, \& Luntz, 2009; Straatman, Huinck, Langereis, Snik, \& Mulder, 2014; van Dijkhuizen et al., 2011; van Dijkhuizen, Boermans, Briaire, \& Frijns, 2016; Waltzman et al., 2002; Watson et al., 2003; Yang et al., 2011; Zeitler et al., 2012). It can be seen in Figure 2 that mean or median postoperative CVC/CNC scores ranged between 12.5 and $46 \%$ for word scores and between 20 and 49\% for phoneme scores (all but 2 studies, see Fig. 2). Postoperative scores were significantly higher than preoperative scores in 8 of the 12 studies (67\%). Five studies in Table 2 reported postop improvements on either phoneme identification (Kos, Deriaz, Guyot, \& Pelizzone, 2009), the PBK/NU6 word-test (Schramm, Fitzpatrick, \& Seguin, 2002) or 2- or 3-syllable words (Bosco et al., 2013; Santarelli et al., 2008; Yang et al., 2011), with scores in the same range as for CVC/CNC words. Sixteen of the studies in Table 2 used an open-set sentence test ${ }^{8}$ in the auditory-

\footnotetext{
${ }^{7}$ Studies for which data are not (fully) presented in Table 2 are (Teoh et al., 2004a) (no mean result for all subjects), Bassim et al. (2005) \& Derinsu, Ciprut, and Akdas (2002) (results not analyzed, not even descriptively), Kaplan, Shipp, Chen, Ng, and Nedzelski (2003) \& Millette, Gobeil, Bherer, and Duchesne (2011) (no preop data nor improvement scores given), van der Marel, Briaire, Verbist, Muurling, and Frijns (2015) (unclear CVC test conditions) and Waltzman, Roland, and Cohen (2002) \& Zeitler et al. (2012) (unclear HINT test conditions).

${ }^{8}$ The sentence tests used included the original or translated versions of the CUNY sentences (City University of New York, Boothroyd, Hanin, and Hnath (1985)), the CID sentences (Central Institute for the Deaf sentences, Davis and Silverman (1978)), the HINT-Q sentences (Hearing In Noise Test - administration in quiet, Nilsson, Soli, and Sullivan (1994)) and the BKB sentences in quiet (Bamford-Kowal-Bench, Bench, Kowal, and Bamford (1979)), as well as a range of less known sentence tests.
} 


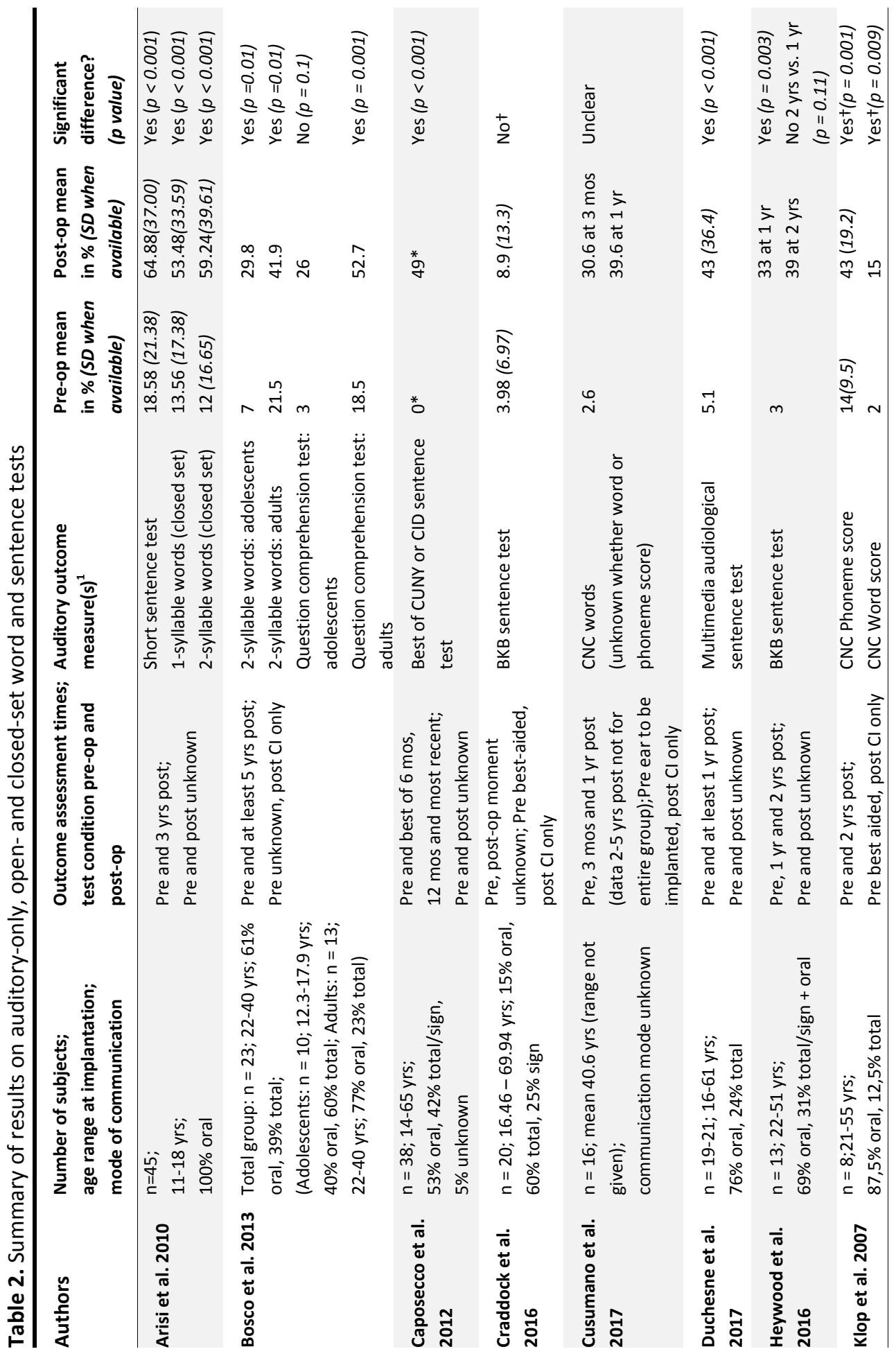




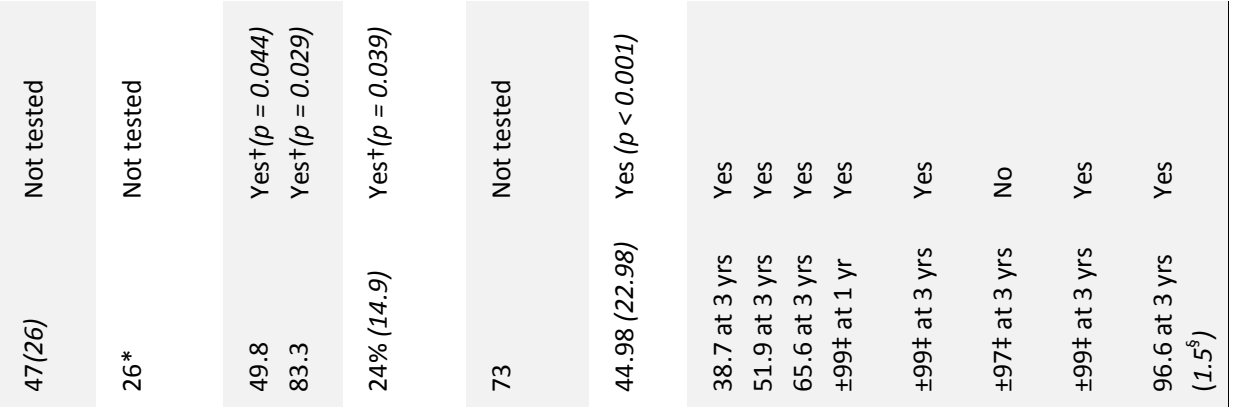

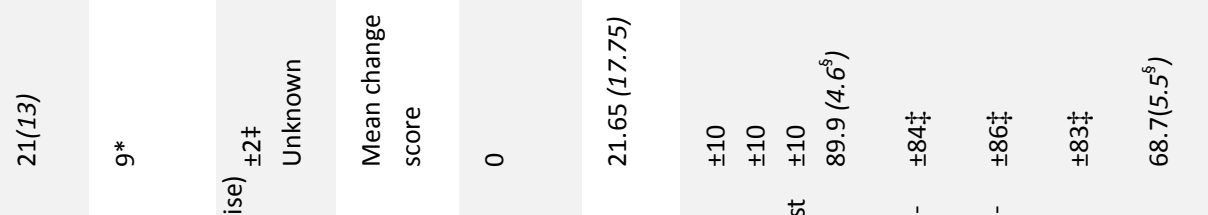

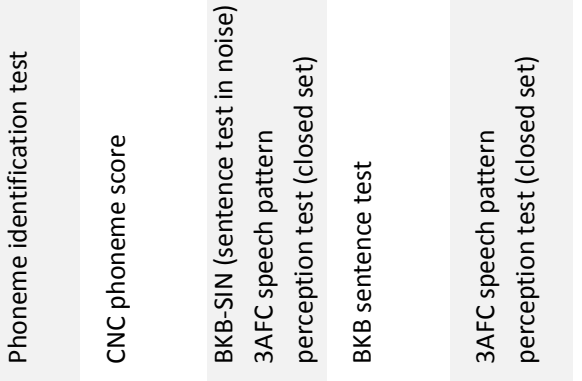

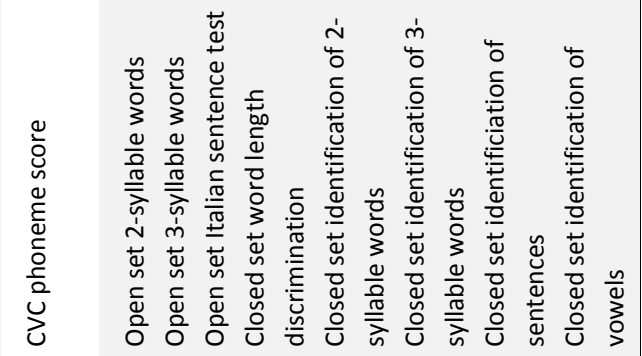

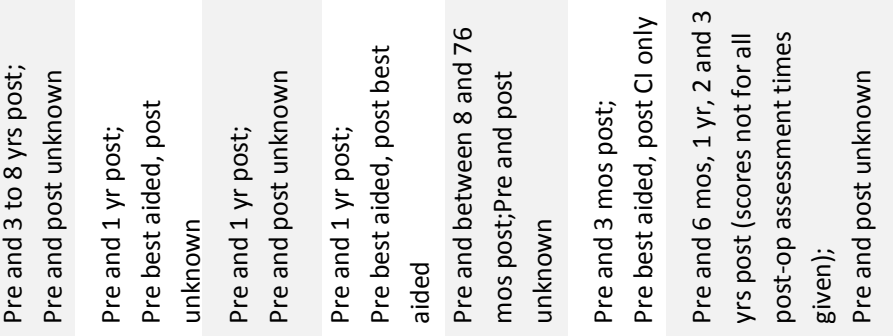

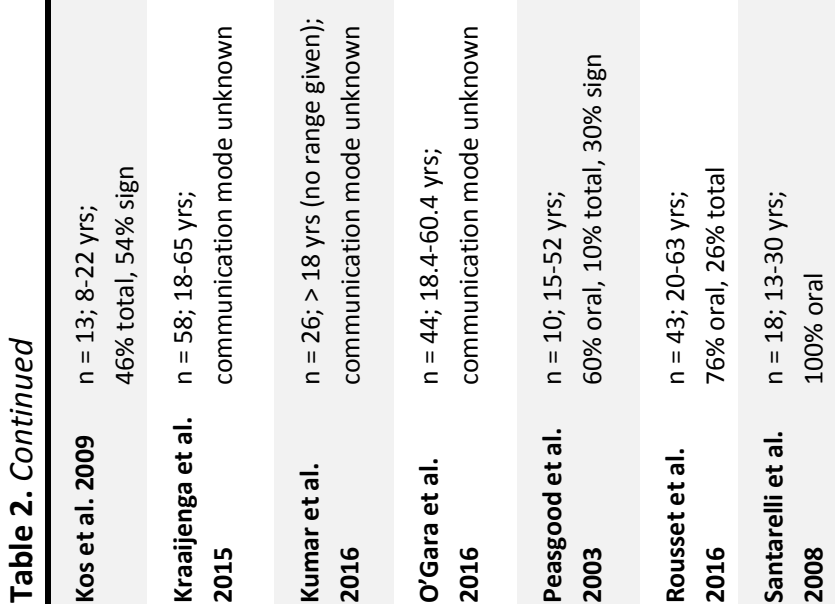


Systematic review part 1: clinical effectiveness

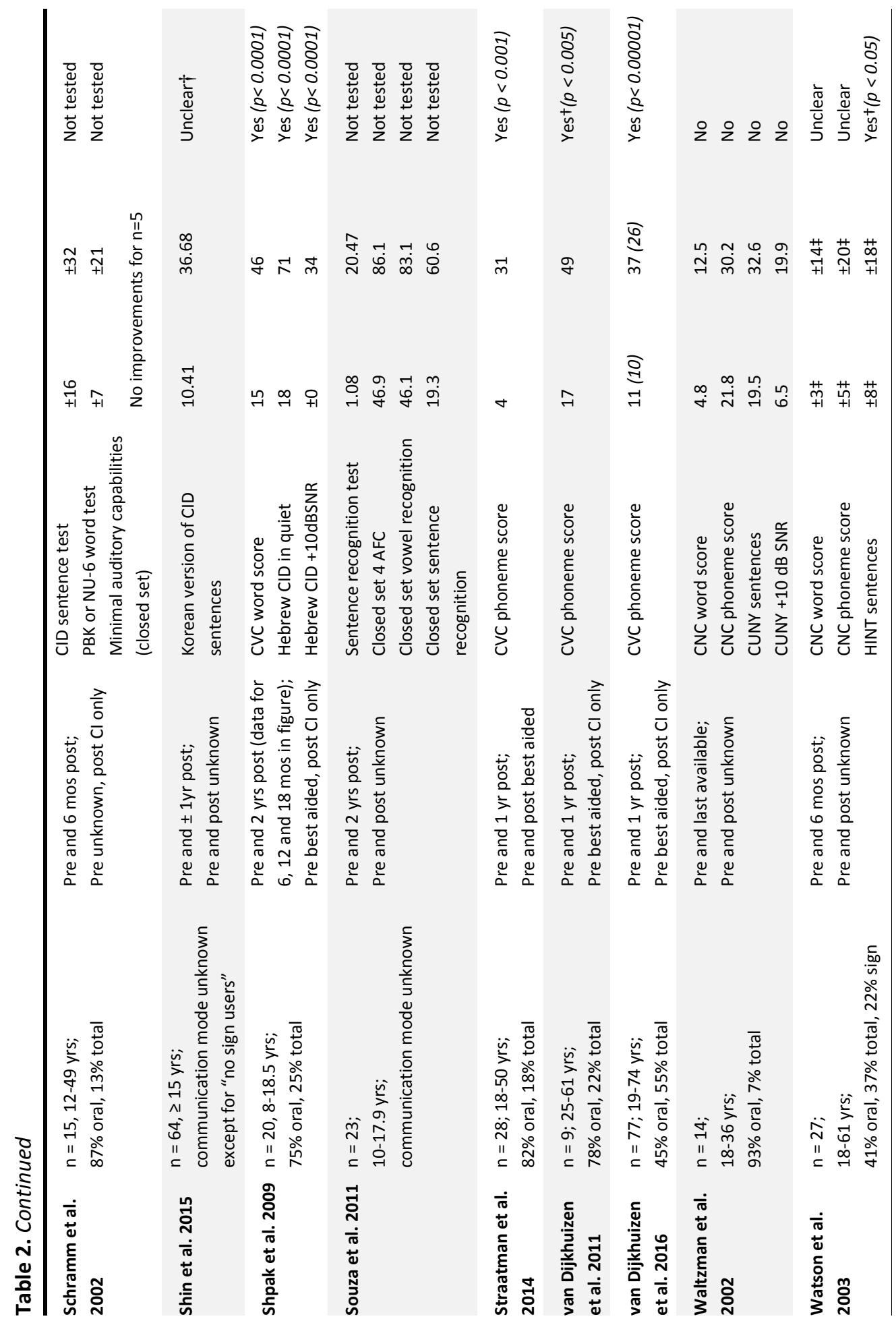




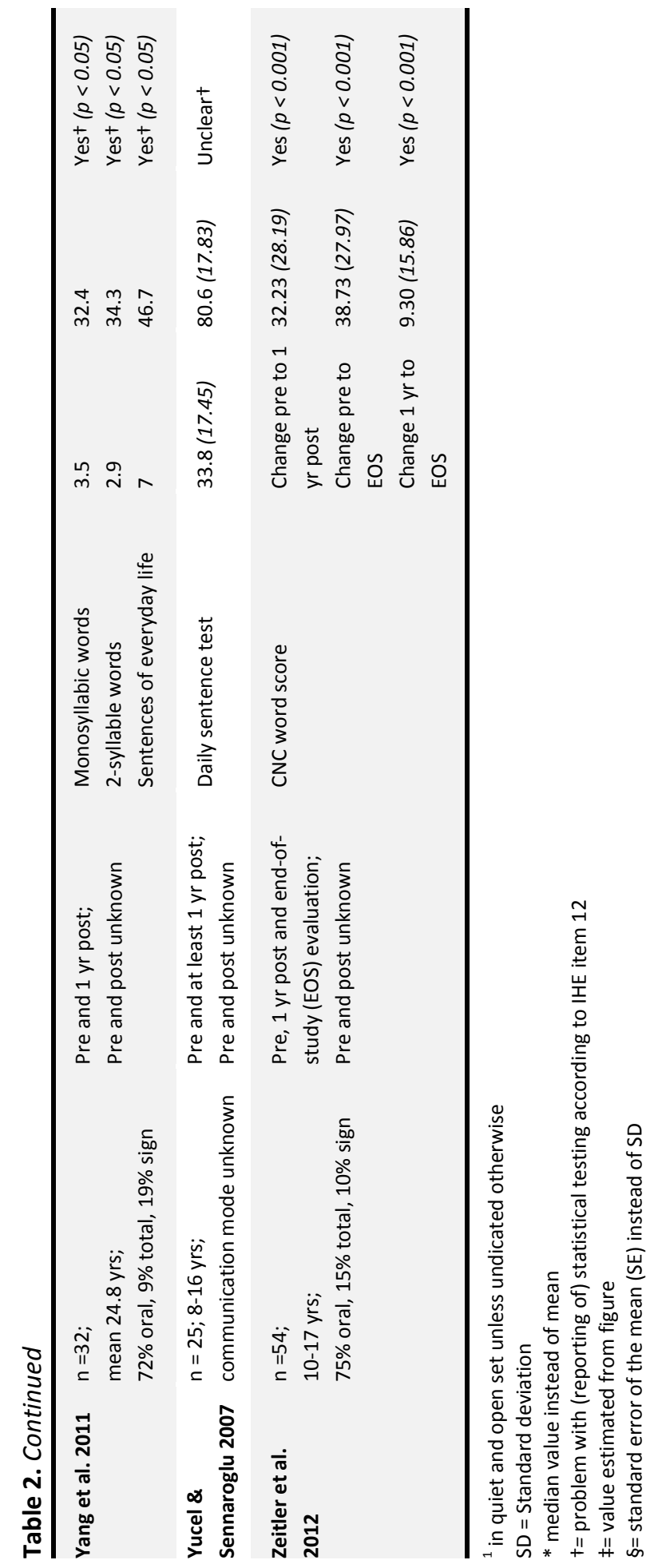




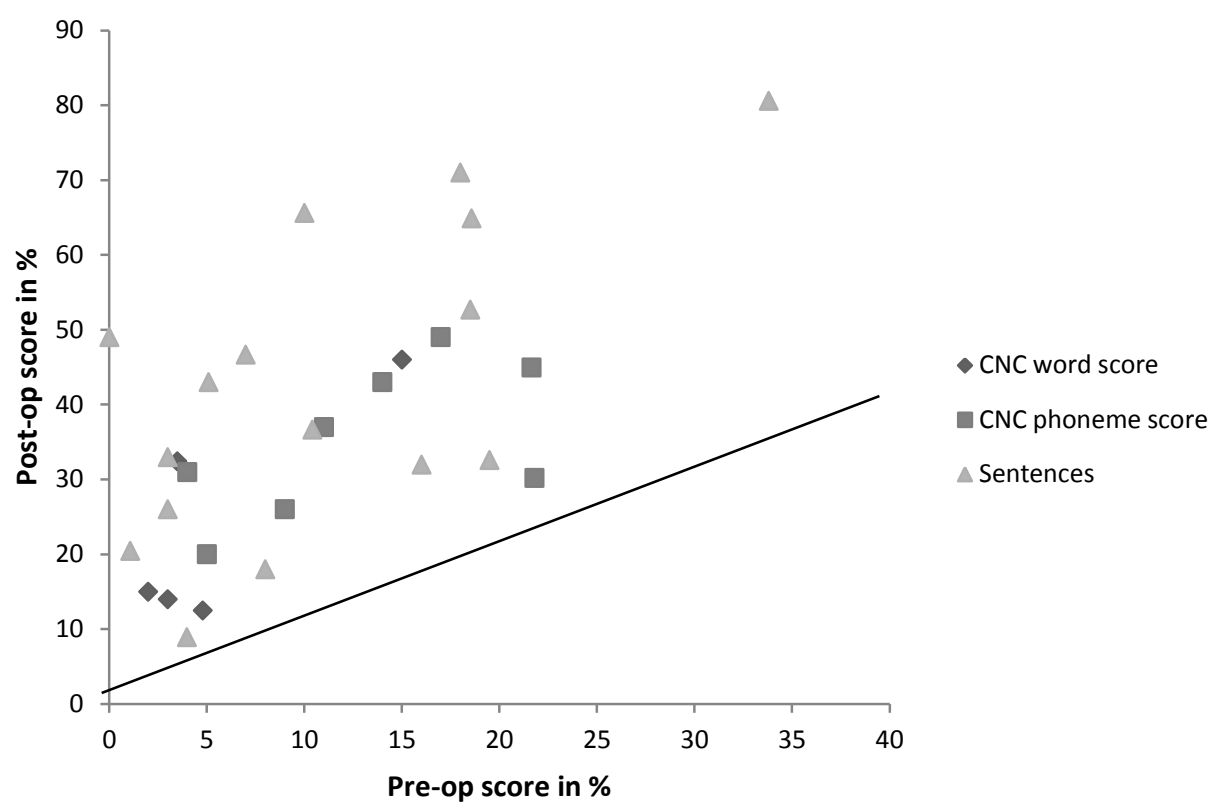

Figure 2. Pre- and postoperative scores on CNC words and sentences in quiet Not included are Cusumano et al. (2017) (unknown whether word or phoneme score), Zeitler et al. (2012) and O'Gara et al. (2016) (only change score given). Scores for the adolescent and adult group in Bosco et al. (2013) are given as 2 separate points.

only condition to evaluate performance. Taking all sentence recognition tests together, mean preoperative scores ranged from 0 to $33.8 \%$ and mean postoperative scores ranged from 8.9 to $80.6 \%$, although in only 5 of the 16 studies the meanpostoperative score exceeded 50\% (Arisi et al., 2010; Bosco et al., 2013 ${ }^{9}$; Santarelli et al., 2008; Shpak et al., 2009; Yucel \& Sennaroglu, 2007), see also Figure 2. The postoperative improvement was significant in 10 of these 16 studies (62.5\%). Where studies in postlingually deafened patients have reported ceiling effects for the traditional sentence tests in quiet (EbrahimiMadiseh, Eikelboom, Jayakody, \& Atlas, 2016; Gifford, Shallop, \& Peterson, 2008; Koch, Osberger, Segel, \& Kessler, 2004), this is clearly not the case for the results presented here. There is therefore no direct need for more difficult testing materials for earlydeafened $\mathrm{Cl}$ users, although this is the general advice of the new Minimum Speech Test Battery for adult cochlear implant users (Auditory Potential, 2011).

In total, 25 studies assessed open-set speech recognition in quiet, 16 of which reported significant postoperative changes. However, the IHE quality assessment (item 12) identified problems with the statistical testing in quite a large number of these 25 studies (see Table 2 and Appendix D). On one hand, there were (minor) problems with the presented statistical results in 5 of the 16 studies that reported a significant change (Klop

\footnotetext{
${ }^{9}$ Adult group only
} 
et al., 2007; O'Gara et al., 2016; van Dijkhuizen et al., 2011; Watson et al., 2003; Yang et al., 2011). On the other hand, problems such as the unclear presentation of statistical results (Cusumano et al., 2017), the use of a wrong statistical test (Shin et al., 2015; Yucel \& Sennaroglu, 2007), and simply the lack of any significance testing for the presented outcomes (Kos et al., 2009; Kraaijenga et al., 2016; Schramm et al., 2002; Souza et al., 2011) also had as a consequence that these studies were not classified as having found a significant change, where they might have done so if adequate statistical testing had been performed. It is therefore likely that the proportion of studies finding a significant change would be higher than the current 16 out of 25 if it were not for these statistical problems, especially given the often large postoperative changes in raw scores of the studies involved. This leaves 3 studies that failed to find a significant postoperative improvement (Bosco et al., 2013 ${ }^{10}$; Craddock, Cooper, Riley, \& Wright, 2016; Waltzman et al., 2002), although there was a problem with the statistical testing in Craddock et al. (2016) as well. In both Waltzman et al. (2002) and Bosco et al. (2013) $)^{10}$, the absence of significant changes might be the result of the combination of a relatively small subject group and small absolute change scores (which we cannot explain for either study), with consequently limited statistical power. In the study by Craddock et al. (2016), mean scores on the BKB sentences in quiet hardly improved (9/20 patients scoring $0 \%$ pre- and postop). A possible explanation for this particularly poor result may lie in the fact that only 3 out of 20 subjects were oral communicators. It is expected that subjects who do not use speech for their day-to-day communication, will score very poorly on an open-set auditory only test, also postimplantation.

Three studies in Table 2 assessed speech understanding of this particular patient group in noise: Shpak et al. (2009) and Kumar et al. (2016) found significant improvements on respectively the Hebrew CID administered at $+10 \mathrm{~dB}$ SNR and the BKB-SIN test (Etymōtic Research, 2005), although the latter did not mention which statistical test was used (see Table 1). On the other hand, Waltzman et al. (2002) did not find a significant increase in mean score on the CUNY, administered at $+10 \mathrm{~dB}$ SNR. This demonstrates that it is possible for some early-deafened $\mathrm{Cl}$ users to obtain some speech understanding in noise, which advocates for a selective use of this type of test in this population.

Six studies in Table 2 evaluated changes after cochlear implantation by means of a variety of closed-set measures: in all but one study mean postop scores easily exceeded $50 \%$, with study groups ranging from 10 to 45 subjects (Arisi et al., 2010; Kumar et al., 2016; Peasgood, Brookes, \& Graham, 2003; Santarelli et al., 2008; Souza et al., 2011). This is notably higher than the chance scores on most of these tests, which were generally in the range of 20-33\%, although the number of alternatives and therefore the chance score was not known for all tests. The improvement was significant in 3 studies (Arisi et al., 2010; Kumar et al., 2016; Santarelli et al., 2008), except for the 3-syllable word identification test

\footnotetext{
${ }^{10}$ Adolescent group only
} 
of Santarelli et al. (2008), which might be due to ceiling effects. Peasgood et al. (2003) and Souza et al. (2011) did not perform significance testing, just like the study by Schramm et al. (2002), which was the only one not to find an improvement on closed-set tests. The fact that these results only concerned a subgroup of 5 subjects who had already failed to show an improvement on open-set tests, and were obtained as soon as 6 months postimplantation, potentially contributes to this. Due to their higher mean postop scores, closed-set tests are generally more suitable for poor performing patients. However, selective use and careful interpretation of these tests is warranted, given their susceptibility to ceiling effects in case of good performers, and the fact that improvements can sometimes be obtained due to chance, even in poor performers.

An alternative way to compare pre- and postimplant performance, by means of hierarchical performance categories, was implemented by 3 studies (see Appendix B). In Caposecco et al. (2012), 68\% of subjects improved by at least one category 12 months postimplantation. On the CAP classification system (Archbold, Lutman, \& Nikolopoulos, 1998), Kos et al. (2009) and Shin et al. (2015) both found postoperative increases in performance level. This type of evaluation system, in which different tests can be administered to different subjects depending on their level of auditory functioning, has the advantage of being much more patient-friendly since it avoids patients having to undergo a battery of tests that are often too difficult and thus frustrating.

Four studies in Table 2 also looked at how performance evolved postoperatively over time. Santarelli et al. (2008) observed that mean scores on various tests continued to increase up to 3 years postop, but since they did not perform - or mention the results - of any additional pairwise comparisons between the different postoperative assessment times this cannot be confirmed statistically (except for the 3 years postop sentence score, which was significantly higher than the 1 year postop score). These authors hypothesize that the continued increase in performance could be attributed to a 'retained plasticity' in the auditory cortical areas of their subjects, given that they were all adolescents with a strong focus on oral communication with hearing aids before implantation. Interestingly, the subjects of 2 other studies that looked into performance over time were equally adolescents or young adults, though not exclusively orally oriented. Zeitler et al. (2012) found that scores improved significantly from 1 year postop to the "end-of-study" evaluation moment (at least 2 years postop), but that the improvement within the first year was significantly greater. On the other hand, Shpak et al. (2009) only found a significant improvement from 18 to 24 months postop for sentences in noise, not for any other test (words or sentences in quiet) beyond 12 months postop. Finally, Cusumano et al. (2017) measured outcomes up to 5 years postop in subjects with a mean age at implantation of almost 41 years: in the bimodal condition results continued to increase significantly up to 3 years postop but not thereafter; their results with $\mathrm{Cl}$ only could not be interpreted straightforwardly (see IHE12). In conclusion, there is no strong statistical evidence that results with $\mathrm{Cl}$ continue to increase for several years following implantation 
in the early-deafened population, though further research is recommended, especially in auditory stimulated subjects that are implanted as adolescents. In order to warrant adequate patient expectations, knowledge on the time frame during which improvements are to be expected is highly relevant.

An unknown part of the variation in the results of the studies presented in Table 2 is likely to arise from variations in how outcomes were assessed, such as differences in the tests used (different validated and unvalidated tests in different languages), the auditory test condition ( $\mathrm{Cl}$ only or best-aided; unknown for most studies), the postop evaluation moment (mostly 1 or 2 years, but as soon as 3 months postop) and a variety of other testing aspects. An even larger source of variation is present between the included subject groups of the studies. Firstly, given that the studies in this review are mostly retrospective (see IHE2, Table 1), and originate from cochlear implant clinics all over the world, the implantation criteria of these centers will determine which patients are included in the subject groups. Second, there are differences with respect to in- and exclusion criteria applied by the studies, although clearly stated eligibility criteria for the study sample are often missing (IHE6). An important aspect here is whether or not data of subjects with a comorbidity are included. Additional disabilities are thought to be present in $30-40 \%$ of children with a hearing-impairment (Johnson \& Wiley, 2009). Since these hearing-impaired children have become the early-deafened adolescents and adult $\mathrm{Cl}$ users in the studies of this review, some of these additional disabilities might have an impact on measured outcomes as well. However, it has been shown in early-implanted children that the effect of mild mental retardation on outcome is limited (Daneshi \& Hassanzadeh, 2007; Holt \& Kirk, 2005) and that the number of additional disabilities is an important determining factor (Nikolopoulos, Archbold, Wever, \& Lloyd, 2008). Of the 27 studies in Table 2, only 8 mention (some form of) comorbidity as an exclusion criterion ${ }^{11}$. Nonetheless, we believe that it is likely that having a comorbidity - especially mental retardation - was an implicit exclusion criterion in a lot of other studies as well. This can for instance be deducted from descriptions of the subject group having adequate motivation (Klop et al., 2007; Peasgood et al., 2003; Yang et al., 2011) or being able to complete additional questionnaires (Duchesne, Millette, Bherer, \& Gobeil, 2017; Heywood et al., 2016; Shin et al., 2015; Straatman et al., 2014). Taken together, although the presence of comorbidity cannot be ruled out as a factor of influence, its impact is not thought to be large.

Apart from the above-mentioned aspects, the variation between included subject groups relates to a number of other items on the IHE quality assessment as well. In some studies, patient recruitment was based on a specific criterion (e.g. sufficient speech intelligibility in van Dijkhuizen et al. (2011); van Dijkhuizen et al. (2016)) (see IHE4) making it more

\footnotetext{
${ }^{11}$ Of these 8 studies, 4 reported that subjects with additional disabilities were excluded (Arisi et al., 2010; Kumar et al., 2016; Yucel \& Sennaroglu, 2007; Zeitler et al., 2012) and another 4 specifically mention mental retardation as exclusion criterion (Bosco et al., 2013; Santarelli et al., 2008; van Dijkhuizen et al., 2011; van Dijkhuizen et al., 2016).
} 
difficult to generalize study results to the average early-deafened population. Another source of variation arises from the inclusion of subjects with a later onset of deafness, an age at implantation $<10$ years or a prelingual, progressive hearing loss (see IHE7). It is also important to note that, with respect to the exact age at onset of deafness and the severity of the hearing loss at the time, reliable information is generally very difficult to obtain. For patients who are now adolescents or adults, it depends on the availability and adequacy of medical records and, especially for older patients, will more likely be based on recall of family members. Yet another aspect inducing variability is that some studies failed to give enough information on relevant patient characteristics (IHE5), making it hard to judge how well the study group represents the population of interest and how study samples differ with respect to communication mode. Postimplant rehabilitation was not described in the majority of studies either (IHE8), although auditory rehabilitation is considered indispensable for an optimal result after cochlear implantation. Finally, although most studies gave results for all included patients, or explicitly reported on subjects lost to follow-up (IHE14), it remains unclear for the majority of studies if and how they dealt with nonusers, a patient group with potentially different outcomes. ${ }^{12}$ Despite these various sources of variation, results on the open- and closed-set speech understanding tests were still fairly cohesive between studies, which makes us suspect that the aspects these studies have in common largely outweigh the various sources of variation presented above.

\section{b. Audiovisual benefit}

Eight studies in this review assessed audiovisual benefit, i.e. the added value of visual cues, made available through lipreading, to speech understanding (for details see Appendix B). A first group of studies compared test results assessed in an audiovisual (AV) condition with those obtained in a solely visual (V) or solely auditory (A) condition. They are interested in how the combination of auditory and visual information relates to the uncombined information, and thus how well both sources integrate with each other. van Dijkhuizen et al. (2011), Craddock et al. (2016) and Moody-Antonio et al. (2005) all found significantly higher scores in the AV condition compared to the lipreading alone (V) condition on different sentence tests. Moody-Antonio et al. (2005) also assessed the magnitude of the relative improvement and found that 3 out of their 8 subjects showed a "superadditive" effect ( $A V>A+V), 3$ subjects showed an "additive" effect $(A V=A+V)$, and 2 subjects showed no effect $(A V \approx V)$. In 2009, Most, Rothem, \& Luntz also found that

\footnotetext{
12 Of the 7 prospective studies in Table 2, 3 clearly reported that there were no nonusers among their participants (Mulder, Straatman, Huinck, Langereis, \& Snik, 2010; van Dijkhuizen et al., 2011; Waltzman et al., 2002) and one mentioned that 2 nonusers were lost to follow-up (van Dijkhuizen et al., 2016). The remainder of the studies in Table 2 had a retrospective or unclear design: only 3 of them mention that nonusers were excluded as subjects (Kraaijenga et al., 2016; Santarelli et al., 2008; Zeitler et al., 2012) and another 5 acknowledge having them in their subject group (Craddock et al., 2016; Duchesne et al., 2017; Kumar et al., 2016; O'Gara et al., 2016; Shpak et al., 2009), but it was not always clear how their data were subsequently handled.
} 
their subjects scored better in the AV condition compared to either A or $\mathrm{V}$ alone (no statistical testing) on both word and sentence tests. They also investigated the superadditive effect, and found that this was commonly present in their subjects for tests where absolute test scores were relatively low, but not for tests prone to ceiling effects: when $\mathrm{A}+\mathrm{V}$ is close to or above $100 \%$, it is no longer possible to attain an even higher $\mathrm{AV}$ score. Most et al. (2009) also suggest that the amount of integrative ability, which determines whether or not a subject attains a superadditive effect, is likely related to general cognitive and language skills as well. The hypothesis that performance with a cochlear implant should be seen as "the endpoint of a large number of complex sensory, perceptual, neurocognitive and linguistic processes" (Pisoni, Kronenberger, Harris, \& Moberly, 2017) is gaining attention recently. It is suggested that differences in the ability of subjects to perform these complex processing tasks are the underlying cause of the large variability in performance between subjects, at least for postlingual adults and earlyimplanted children. In subjects with early-onset deafness, their reduced auditory experience will not only affect language development, but also have implications for the development of different aspects of cognitive functioning, such as working memory and attention (Kral, Kronenberger, Pisoni, \& O'Donoghue, 2016). In addition, without early restoration of auditory stimulation, top-down, cognitive modulations on auditory perception are equally reduced (Kral et al., 2017). Subjects that demonstrate a superadditive effect therefore seem able to integrate auditory and visual information to a certain extent, on a higher cognitive level, despite these limitations. It would thus be interesting to investigate whether these subjects have better outcomes on general measures of sensory processing as well, as would be expected according to the hypothesis of Pisoni et al. (2017).

A second group of studies on audiovisual communication compared pre- and postoperative outcomes on AV tests, with visual information being present both pre- and postop. O'Gara et al. (2016), Kumar et al. (2016) and Heywood et al. (2016) all found significant postoperative improvements on the CUNY sentences with lipreading, as well as Duchesne et al. (2017) on the AV condition of the Multimedia Audiological Test. In contrast, Craddock et al. (2016) found no significant change in AV performance on the CUNY sentences, preop with hearing aids and postop with $\mathrm{Cl}$-alone. Potential explanations are the small proportion of oral communicators in this study (only 15\%), poor open-set sentence recognition performance of their subjects (mean $8.9 \%$ ) and the fact that postop AV performance was measured with $\mathrm{Cl}$ alone, not allowing for the contribution of a contralateral hearing aid. When assessing audiovisual benefit, for which the closer resemblance to everyday communication is a key point, it seems most logical to perform the test in the best-aided condition both pre- and postoperatively. However, only O'Gara et al. (2016) mentioned that this was the case in their study; the remaining 3 studies that observed a significant postop improvement (Duchesne et al., 2017; Heywood et al., 2016; Kumar et al., 2016) left it unclear whether a contralateral hearing aid could be used 
postoperatively or not. This lack of sufficient details on the specific test condition makes it more difficult to interpret and compare study outcomes and is a recurring point of attention.

The IHE quality assessment identified some additional shortcomings in the majority of the 8 studies on audiovisual benefit. These shortcomings include: part of the subjects having an onset of deafness beyond 4 years of age (Craddock et al., 2016; Heywood et al., 2016; Peasgood et al., 2003), only a small number of eligible patients actually participating in the study (Moody-Antonio et al., 2005), eligibility criteria not mentioned (Craddock et al., 2016; Most et al., 2009; Peasgood et al., 2003), relevant patient characteristics not described (Kumar et al., 2016) and insufficient information given on the statistical tests and whether the assumptions for these tests were met (Craddock et al., 2016; Kumar et al., 2016; O'Gara et al., 2016).

In conclusion, results of the included studies showed that early-deafened subjects were generally able to combine the new ( $\mathrm{Cl}$ ) with the old (lip reading and/or hearing aid) information, sometimes even to the extent of a "super-additive" effect, with subjects gaining access to a much higher level of speech understanding.

\section{c. Subjective benefit and health-or hearing-related quality of life}

Sixteen studies in this review used one or more measures to assess subjective benefit of cochlear implantation in prelingual patients. In 5 of those, results on a validated or previously published questionnaire, concerning hearing-related quality of life, were presented. On the International Outcome Inventory for $\mathrm{Cl}$ (IOI-CI, adapted from the IOI-HA by Cox and Alexander (2002)), Caposecco et al. (2012) found median scores of 4 or 5 for their 23 subjects on the 5-item response scales of the outcome domains. On the PIPSL (Performance Inventory for Profound and Severe Loss (Owens \& Raggio, 1988)), Schramm et al. (2002) found higher scores 6 months postop compared to preop, on 4 out of 6 functional categories (see Appendix B), although this was not statistically confirmed. Postoperative changes were lacking for the categories "speech with visual cues" and "personal"; according to the authors, the first can be explained by the fact that most oral $\mathrm{Cl}$ users in their study were already very skilled speech readers before the implantation, not likely to experience any further improvements. Also along this line, the way patients feel about their hearing (category "personal") might not change due to the life-long existence of a profound hearing loss. However, the fact that results on the PIPSL were available for a very small subgroup of 7 (out of 15 ) subjects, is likely to be of influence as well. In 3 studies the Nijmegen Cochlear Implant Questionnaire or NCIQ (Hinderink, Krabbe, \& Van Den Broek, 2000), a validated instrument to measure hearing-related QoL in $\mathrm{Cl}$ users, though not specifically early-deafened patients, was used. Most recently, Straatman et al. (2014) found significant postoperative improvements for a group of 20 subjects on all 6 domains (basic sound perception, advanced sound perception, speech production, self-esteem, activity and social interaction). In 2011, van Dijkhuizen et al. 
found significant improvements on all but one domain (speech production) in a group of 9, measured at least 1 year postop. Finally, Klop et al. (2007) assessed changes on the NCIQ for their 8 subjects, as soon as 4 and up to 30 months postop. They observed significant improvements at 4 months on only $3 \mathrm{NClQ}$ domains, with no more changes thereafter. For the latter 2 studies, the lack of significant improvements on part of the domains of the $\mathrm{NCIQ}$ could be the result of the small subject numbers. In addition, statistical testing was not entirely appropriate either (see IHE12).

In another 5 studies validated, though not hearing-specific, measures of general health status were used: the GBI (Glasgow Benefit Inventory, Robinson, Gatehouse, and Browning (1996)), HUI2 (generic Health Utilities Indices Mark 2, Torrance et al. (1996)), HUI3 (generic Health Utilities Indices Mark 3, Feeny, Furlong, Saigal, and Sun (2004)) and SF-36 (SF-36 health survey, Ware and Sherbourne (1992)) questionnaires. The GBI retrospectively evaluates the effect of an otolaryngological surgical procedure on the health status of a patient. It was used by 4 studies in this review. Peasgood et al. (2003) observed a mean overall benefit score of 38.9 in their 10 subjects. Caposecco et al. (2012) found a positive total health score of 49.4 in a group of 20. Straatman et al. (2014) found a total GBI score around 25 , which was a significant improvement. In the latter 2 studies the greatest benefit was seen for the general health subscale, followed by the social support scale, and there was no change in the physical health subscale. This seems logical since the general subscale contains questions on self-consciousness and social activities, whereas the physical health subscale assesses medicine use and doctor visits, which is not thought to be affected by $\mathrm{Cl}$. The GBI results reported by Kumar et al. (2016) cannot be interpreted straightforwardly. The HUI2 or HUI3 questionnaires were used in 3 studies to evaluate general health status before and after $\mathrm{Cl}$. Results presented by Kumar et al. (2016) were again unclear, and are not incorporated. Klop et al. (2007) only observed a significant improvement on the domain sensation of the HUI2, already 4 months postop with no more changes up to 30 months postop. Straatman et al. (2014) found significant improvements on the subdomains hearing and emotion of the HUI3 in a group of 20 subjects 1 year postop, as well as a significant improvement for the general utility score (i.e. summary score). Finally, one study (Bellelli, Forli, Berrettini, \& Turchetti, 2016) found significant changes on 3 of the 8 domains of the SF-36 in 30 prelingual subjects (general health, social functioning and mental health). The general health questionnaires in earlydeafened subjects thus mainly show a positive effect of $\mathrm{Cl}$ for domains that are related to audition and psychological wellbeing.

The largest group of studies (10 out of 16) however, consists of studies that retrospectively assessed subjective benefit after cochlear implantation by means of unvalidated questionnaires or structured interviews. Their outcomes can be called very positive. Five studies were directly interested in the degree of satisfaction with the implant: 3 report that around $90 \%$ of subjects or more were at least moderately satisfied with the implant (Bosco et al., 2013; Caposecco et al., 2012; Chee et al., 2004), whereas 2 
other studies, both using a French adaption of the Adult Cochlear Implant Questionnaire originally by Zwolan et al. (1996), report satisfaction rates around 70\% (Duchesne et al., 2017; Millette et al., 2011). In the latter two studies, Cl usefulness was assessed as well, with around $70 \%$ of subjects judging their implant to be generally or very useful. Nonusers and part-time users of the $\mathrm{Cl}$ had lower scores. A number of other studies found that around $90 \%$ of subjects would choose to be implanted again (Bosco et al., 2013; Chee et al., 2004; Watson et al., 2003). Chee et al. (2004) and Kaplan et al. (2003) found that respectively $96.7 \%$ and $94.7 \%$ of their subjects indicated that the $\mathrm{Cl}$ had a positive impact on their quality of life. Patients in the study by Chee et al. (2004) listed family and peer support, as well as prior auditory-verbal therapy, as the most important factors contributing to successful implant use. In 2016, Heywood et al. reported that the selfdefined expectations were fully met in 11 of their 13 subjects. The expectations of the $\mathrm{Cl}$ listed most often by these subjects were better communication and hearing more sounds, followed by aid in lipreading, hearing one's children, socializing and more self-confidence. The domains in which positive changes were reported throughout the various studies were, amongst others, communication mode (Bosco et al., 2013) and communicative skills (Chee et al., 2004; Kumar et al., 2016; Mantokoudis et al., 2011), communication when aided by lipreading (Caposecco et al., 2012; Duchesne et al., 2017; Kumar et al., 2016), self-esteem and self-confidence (Bosco et al., 2013; Chee et al., 2004; Duchesne et al., 2017), perception of environmental sounds (Caposecco et al., 2012; Duchesne et al., 2017), perception of personal safety (Duchesne et al., 2017), social life (Chee et al., 2004), and use of the telephone (Caposecco et al., 2012). The fact that the questionnaires or interviews used were mostly specifically designed for the study at hand and consequently not validated, is reflected in the IHE quality assessment (item 10). Although generally not stated, the choice for an unvalidated outcome measure is likely driven by the limited availability of validated quality of life instruments that can be used to assess the effect of an intervention such as cochlear implantation, especially in this very specific population. The choice for (structured) interviews instead of - or in addition to - questionnaires can be explained by the limited level of reading comprehension of this specific population. In addition, the questions posed in these measures might be better adjusted to the areas in which early-deafened subjects experience benefit, instead of the benefits more usually reported for postlingual $\mathrm{Cl}$ users (e.g. questions on telephone use are likely to be irrelevant for most patients). In the case of interviews, the areas in which a patient might experience benefit are not predefined as in a questionnaire, but put forward by the patient itself. Therefore, the unvalidated instruments might even have certain advantages when it comes to capturing the effect of cochlear implantation on quality of life within the early-deafened population.

An important issue, however, is that for those retrospective measures that directly or indirectly assess patient satisfaction, which is the case for most of the unvalidated measures presented above, the outcome will to a large extent be determined by whether 
the preoperative expectations of a patient were met (e.g. Hamilton et al. (2013)). Patients that had low expectations and did not improve their auditory functioning very much, can still be very satisfied $\mathrm{Cl}$ users, whereas patients that did improve their auditory functioning but had very high expectations can be dissatisfied $\mathrm{Cl}$ users. The validated pre-post measures of auditory quality of life, such as the $\mathrm{NCIQ}$, are much less susceptible to the influence of patient expectations, as they do not ask patients to make a direct comparison with a previous situation. Although it is important for patients to be satisfied with their cochlear implant, we should be aware of the influence of expectations and aim for a holistic approach when evaluating the outcome of cochlear implantation in this population, where objective measures of auditory functioning and subjective measures of hearing-related quality of life and patient satisfaction are taken into account.

Related to this issue, four of the 16 studies additionally assessed whether the observed outcomes for subjective benefit were in any way related to speech perception outcomes. The results of (Millette et al., 2011) (see IHE12) and Kumar et al. (2016) unfortunately had unclear results on this topic and could not be used. Therefore the results of 2 studies remain: in 2014, Straatman et al. could not find a significant correlation between benefit in phoneme scores and the postoperative changes on the $\mathrm{HUI}-3, \mathrm{GBI}$ or the $\mathrm{NCIQ}$, whereas in 2017, Duchesne et al. observed a number of significant correlations, mainly between auditory sentence recognition scores and the number of situations where subjects found the implant to be useful, as with the number of changes subjects observed in 7 areas of life (see Appendix B). The fact that the specific objective and subjective outcome measures used greatly differed between both studies, makes it hard to compare the results. More research is therefore needed, preferably using validated outcome measures which are used in large groups of patients, in order to clarify the extent and nature of the relation between subjective benefit and speech perception. The current lack of strong evidence for such a relation emphasizes the need for a holistic approach towards outcome evaluation, as stated before.

Another issue when assessing subjective benefit and changes in quality of life is that, when measured shortly after the end of the rehabilitation period, the positive attention someone received during this period could increase the perceived benefit of the implant. In most studies in this review subjective benefit was assessed only once (range: 6 months 9 years postop), and the assessment point often differed between subjects within the same study as well. In only one study, data on subjective benefit was clearly presented for multiple assessment times: Klop et al. (2007) observed significant changes which remained stable up to 30 months postop on the $\mathrm{HUI} 2$ and $\mathrm{NCIQ}$, although their choice of statistical tests was not entirely appropriate. This result, combined with the mostly significant changes of various studies with variable outcome assessment times, suggests that changes in quality of life are independent of the rehabilitation period and are sustained over time. Finally, the quality assessment of the studies presenting data on subjective benefit or quality of life showed that there are uncertainties or problems with respect to how 
patient recruitment took place in about half of them (see IHE4) (Bosco et al., 2013; Duchesne et al., 2017; Heywood et al., 2016; Klop et al., 2007; Kumar et al., 2016; Mantokoudis et al., 2011; Millette et al., 2011; van Dijkhuizen et al., 2011; Watson et al., 2003). In addition, the number of subjects with available data on the subjective outcome measures is smaller than the number of included subjects in at least 7 of the 16 studies, due to a limited response rate (Caposecco et al., 2012; Chee et al., 2004; Kaplan et al., 2003; Millette et al., 2011; Schramm et al., 2002; Straatman et al., 2014; Watson et al., 2003): this problem occurs since most questionnaires are sent out through mail. If the subjects not returning the questionnaire differ systematically from the subjects that do, the outcome is affected by nonresponse bias. For the studies at hand, it is probable that subjects who are not so satisfied with their implant are less likely to go through the effort of completing and returning a questionnaire, which might lead to an overly positive picture of subjective benefit and hearing-related quality of life after cochlear implantation in this population.

\section{Limitations of the current review}

Although a substantial number of studies were included, the imposed language limits and problems tracing the full-texts of a number of search results (namely theses), potentially limited the representability of the outcomes. In addition, relevant information on various study aspects was not clearly reported in quite a number of studies, leading to difficulties interpreting the results and drawing conclusions. It might have been better to use a cutoff criterion based on the quality assessment, in order to exclude low quality studies, especially since (part of) the results of these studies could not be used after all. Finally, due to insufficient quality of several studies and very heterogeneous outcome measures, it was not feasible to pool outcomes for meta-analysis or effect size calculation.

\section{SUMMARY AND FINAL CONSIDERATIONS}

The current systematic review aimed to give an overview of the outcomes of earlydeafened, late-implanted adults or adolescents with a state-of-the-art cochlear implant, with respect to postoperative outcomes on open- and closed-set speech understanding, audiovisual benefit and subjective benefit. Quality of the included studies was assessed by means of the IHE tool for case series studies.

Results showed that a cochlear implant significantly increased open-set speech perception in quiet in 16 of the 25 studies involved, although this number could have been higher if adequate statistical testing had been performed in all studies. Despite differences between subjects groups and testing conditions, postoperative outcomes were quite homogeneous and generally remained below $50 \%$. In comparison, mean word recognition scores in postlingual $\mathrm{Cl}$ users have repeatedly been reported to lie around 60\% (Cusumano et al., 2017; Gifford et al., 2008; Holden et al., 2013; Kraaijenga et al., 2016; Medina et al., 
2017). Looking at closed-set measures, post-op scores easily exceeded $50 \%$ and therefore chance scores, making them more suitable for follow-up of poorer performing earlydeafened $\mathrm{Cl}$ users. In addition, mean auditory-visual speech recognition performance improved after implantation in almost all studies assessing this, which is highly relevant since early-deafened subjects often rely heavily on visual cues for their day-to-day communication. The largest improvements in auditory performance occurred within the first year after implantation, and there is currently not enough evidence to support the hypothesis that performance of these patients continues to increase significantly beyond this point. The outcomes of this review are clearly more positive than those of Teoh et al. (2004a), with significant improvements for mean open-set speech recognition in the majority of studies and very good outcomes for closed-set speech recognition.

Studies assessing subjective benefit after cochlear implantation in this patient group mainly used a wide variety of unvalidated instruments, the results of which were abundantly positive. A much smaller proportion of studies used validated, hearing-related questionnaires such as the $\mathrm{NCIQ}$. The latter generally showed significantly higher scores postimplantation, though not always for all domains. Unfortunately none of the studies in this review used a validated, hearing-related questionnaire focusing on the specific characteristics of early-deafened $\mathrm{Cl}$ users. Further research is also needed to clarify the relation between subjective outcomes and speech perception, since current results did not allow for any conclusions to be drawn. It is therefore advised to take both measures of speech understanding and hearing-related quality of life into account when evaluating the outcome of cochlear implantation in this patient group.

The interpretation of the various study outcomes was complicated by several methodological issues, most of which were identified by means of the IHE quality assessment. Recurring issues were a lack of information on a number of relevant study aspects, from inclusion criteria to outcome assessment method, and inadequate statistical testing, which compromised the strength of the presented evidence on one hand and potentially limited the number of studies observing significant changes on the other. Variation between the included study groups of the different studies will also have confounded the observed results, but it is hard to judge to which extent. It is recommended that future studies on $\mathrm{Cl}$ in this population have a prospective design, with an exhaustive description of the eligibility criteria, which are also the implantation criteria, pay attention to a comprehensive description of all relevant study variables and perform adequate statistical analyses.

In conclusion, this review has shown that open-set speech recognition can significantly improve after cochlear implantation in early-deafened but late-implanted adults. As mean open-set scores remain low, it is important that outcome evaluation in this population also includes measures of closed-set and audiovisual speech recognition, for which substantial improvements were demonstrated as well. In addition, significant changes after implantation were found for hearing-related quality of life. The interpretation of the 
Systematic review part 1: clinical effectiveness

various study results was unfortunately complicated by several methodological problems, which emphasizes the need for more high-quality research in this patient population. 


\section{Chapter 4}

\section{APPENDIX A: Search syntax per database}

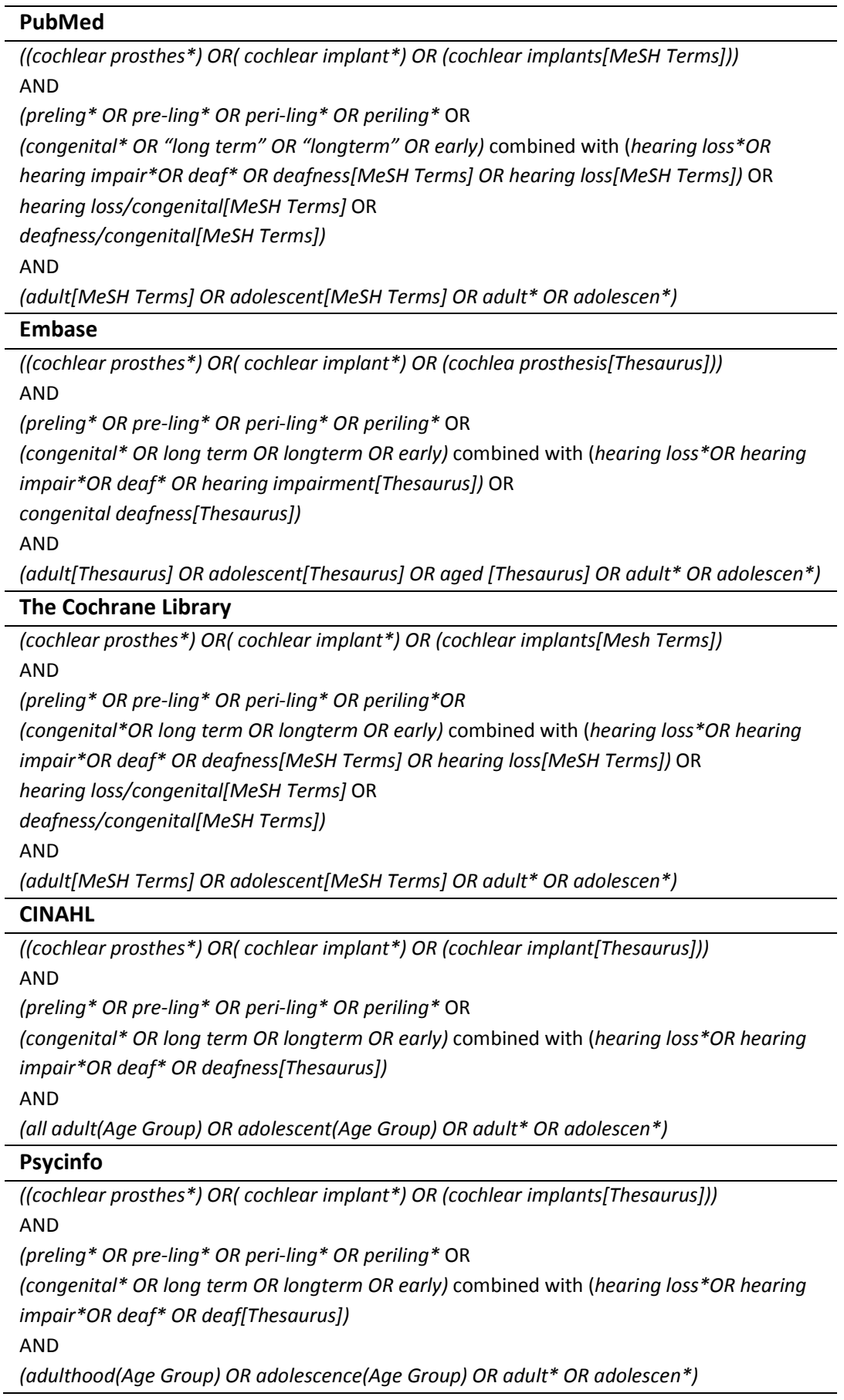




\section{APPENDIX B: General Summary of the studies included in Part 1}

\begin{tabular}{|c|c|c|c|c|c|}
\hline 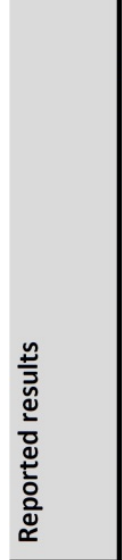 & 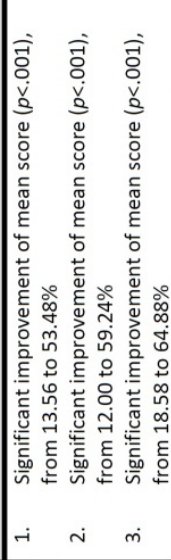 & 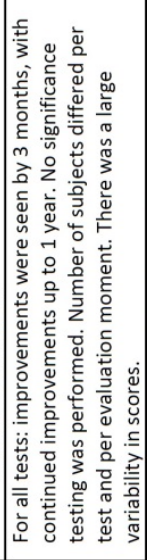 & 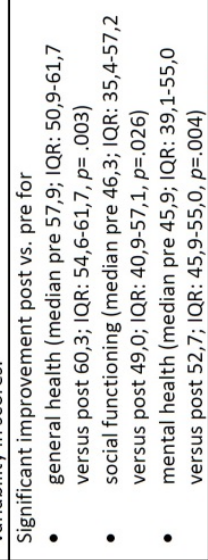 & 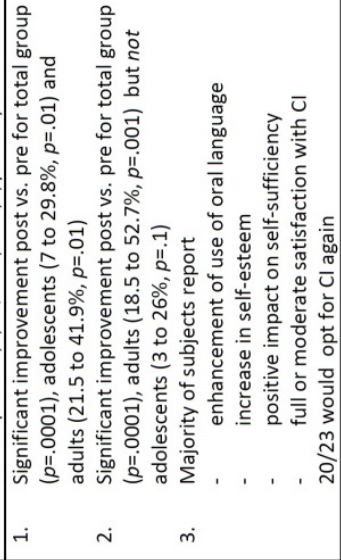 & 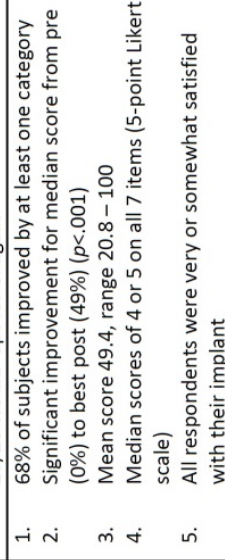 \\
\hline 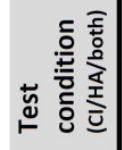 & \begin{tabular}{|l} 
\\
$\frac{5}{3}$ \\
0 \\
0 \\
$\frac{5}{5}$ \\
$\frac{5}{5}$
\end{tabular} & \begin{tabular}{|l}
$\frac{5}{3}$ \\
0 \\
$\frac{5}{5}$ \\
5.
\end{tabular} & 总 & 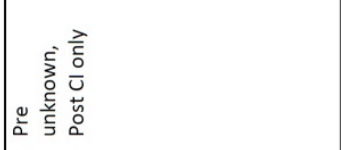 & $\mid$\begin{tabular}{|l}
$\frac{5}{3}$ \\
0 \\
$\frac{5}{5}$ \\
5 \\
5
\end{tabular} \\
\hline 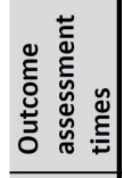 & 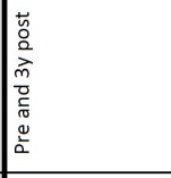 & 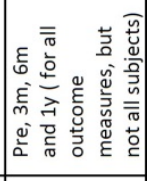 & 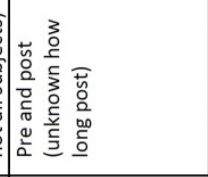 & 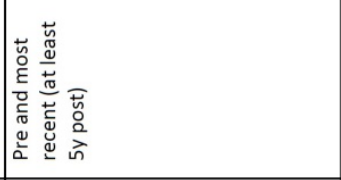 & 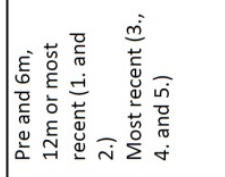 \\
\hline 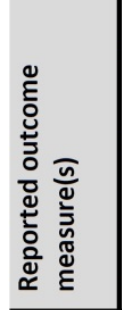 & 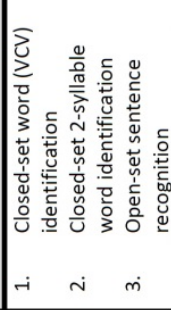 & 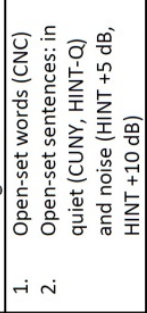 & 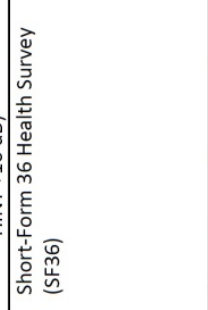 & 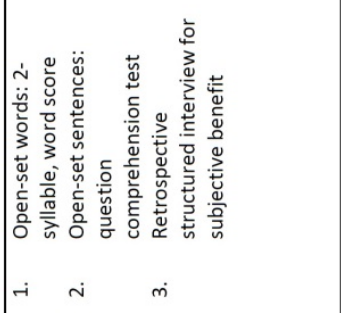 & 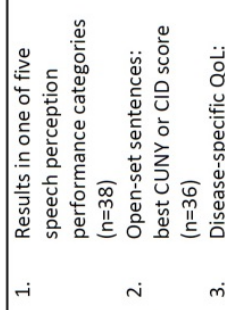 \\
\hline 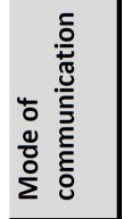 & 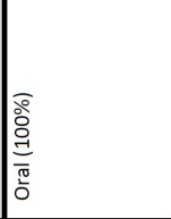 & $\mid \begin{array}{l}\frac{5}{3} \\
\frac{5}{5} \\
\frac{5}{5}\end{array}$ & 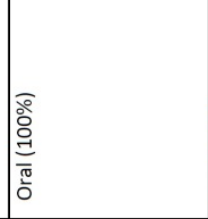 & 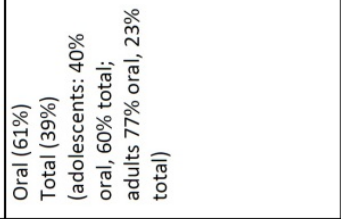 & 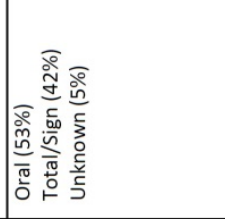 \\
\hline 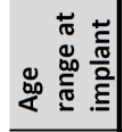 & 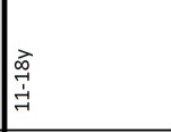 & $\begin{array}{l}\text { 悥 } \\
\text { స్ }\end{array}$ & 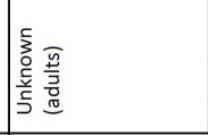 & 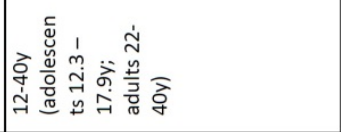 & $\begin{array}{l}\text { ڤ్ర } \\
\vdots \\
\text { d }\end{array}$ \\
\hline 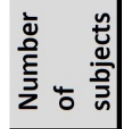 & \& & $\approx$ & p & 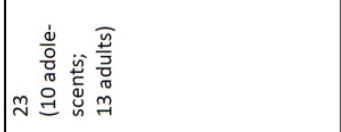 & $\prod_{\infty}^{\infty}$ \\
\hline 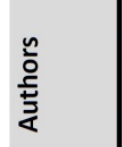 & 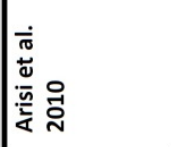 & 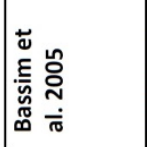 & 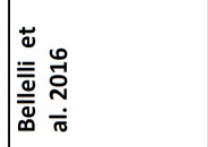 & 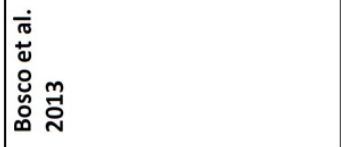 & 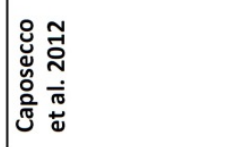 \\
\hline
\end{tabular}




\section{Chapter 4}

\begin{tabular}{|c|c|c|c|c|}
\hline & 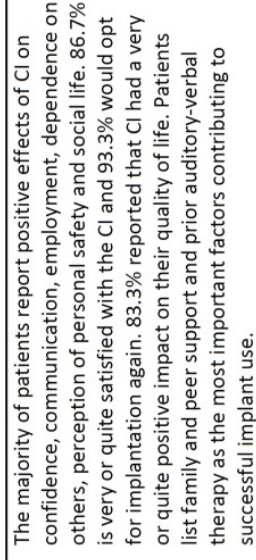 & 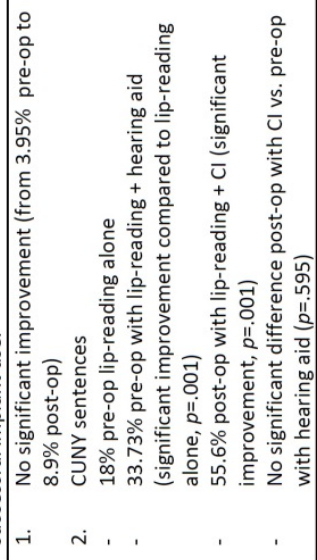 & 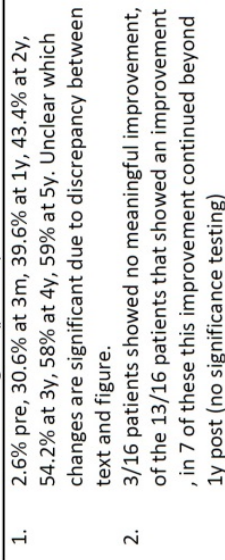 & 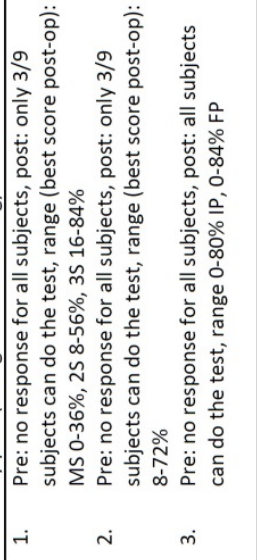 \\
\hline & 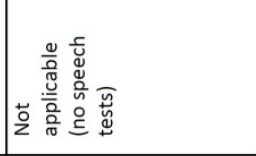 & 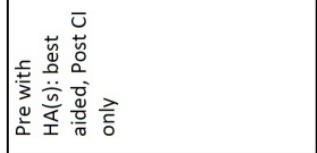 & \begin{tabular}{|l}
$\frac{\lambda}{\bar{c}}$ \\
$\frac{\bar{\sigma}}{\mathrm{U}}$
\end{tabular} & 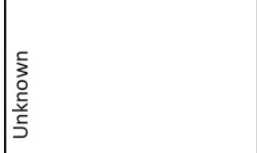 \\
\hline & 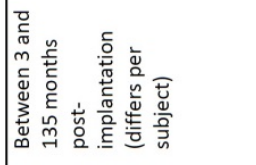 & 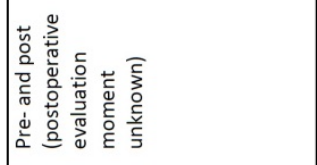 & 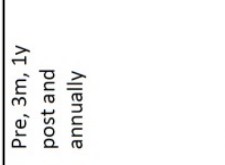 & 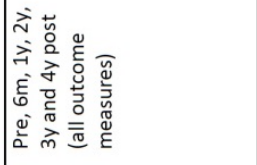 \\
\hline 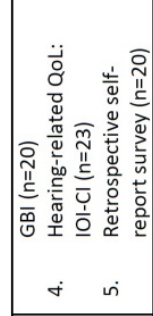 & 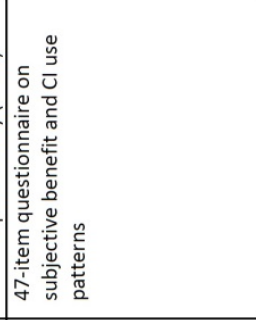 & 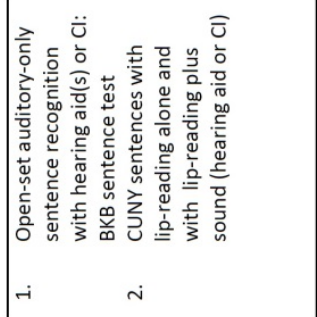 & 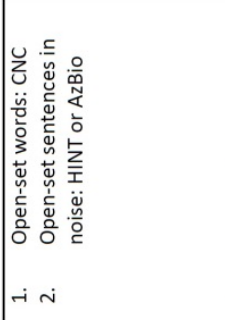 & 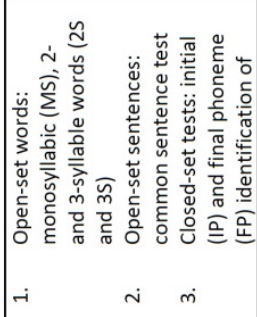 \\
\hline & \begin{tabular}{|l}
5 \\
$\frac{5}{0}$ \\
0 \\
$\frac{5}{5}$ \\
5
\end{tabular} & 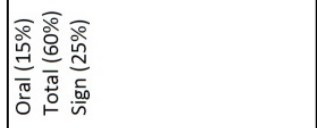 & \begin{tabular}{|l}
$\frac{5}{3}$ \\
0 \\
$\frac{5}{5}$ \\
$\frac{5}{5}$
\end{tabular} & 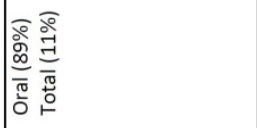 \\
\hline & 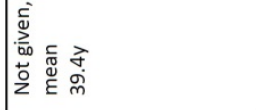 & 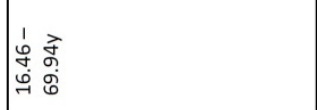 & 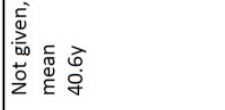 & 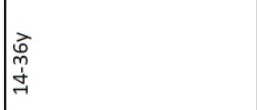 \\
\hline & 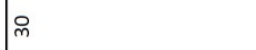 & i & $\stackrel{1}{\pi}$ & a \\
\hline & 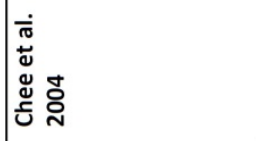 & 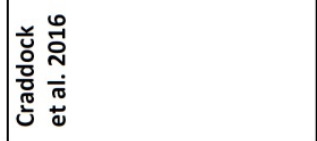 & 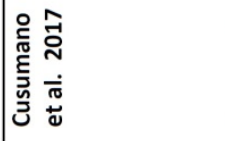 & 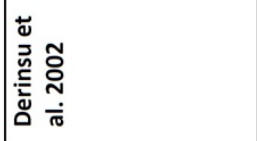 \\
\hline
\end{tabular}


Systematic review part 1: clinical effectiveness

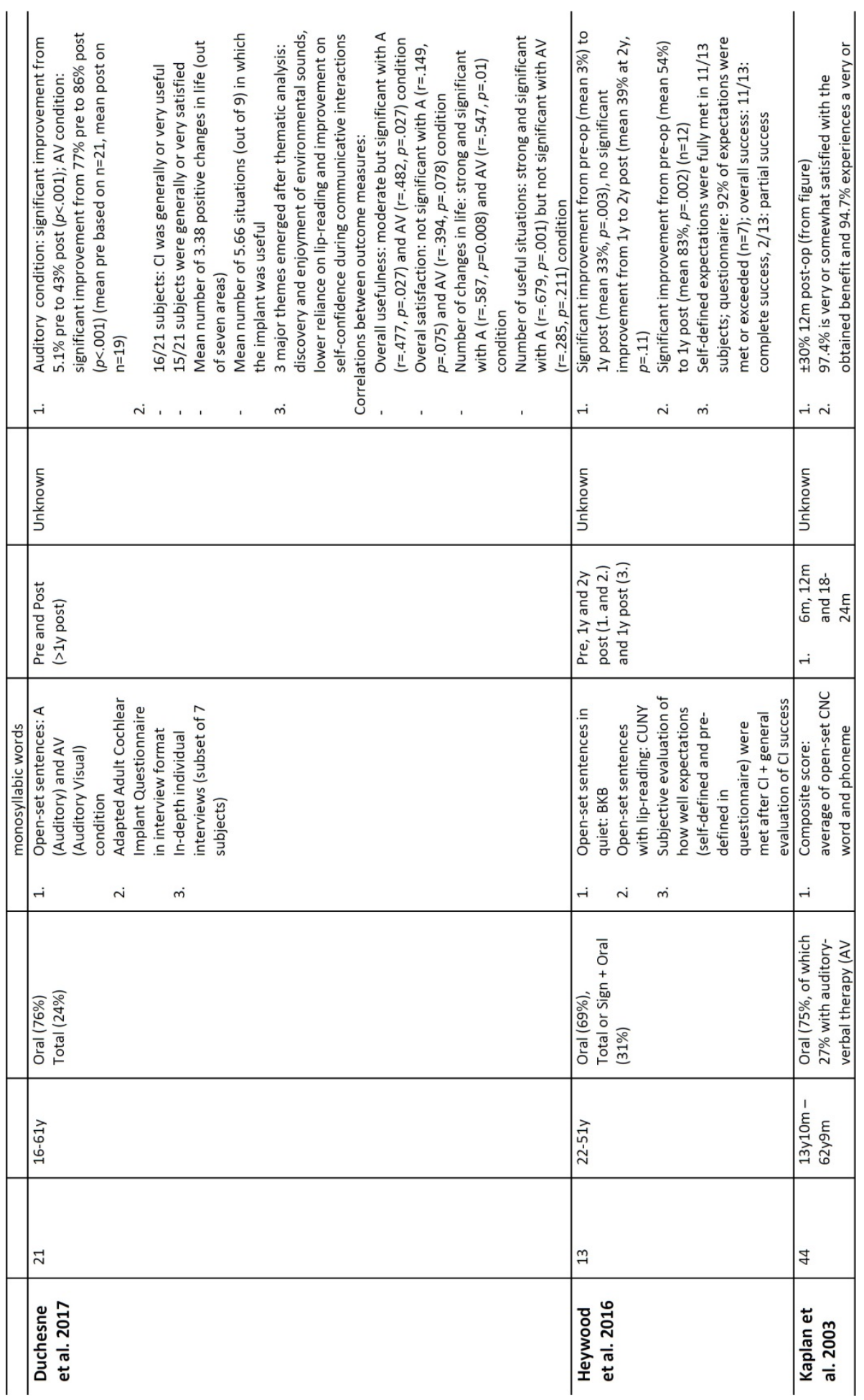




\section{Chapter 4}

\begin{tabular}{|c|c|c|c|c|}
\hline & 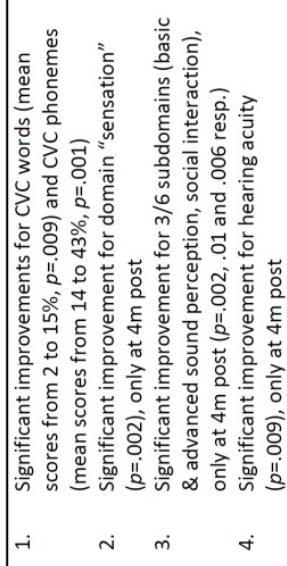 & 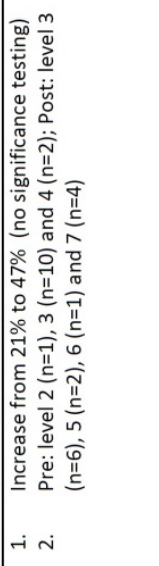 & 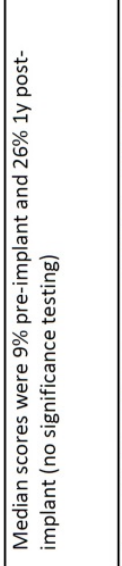 & 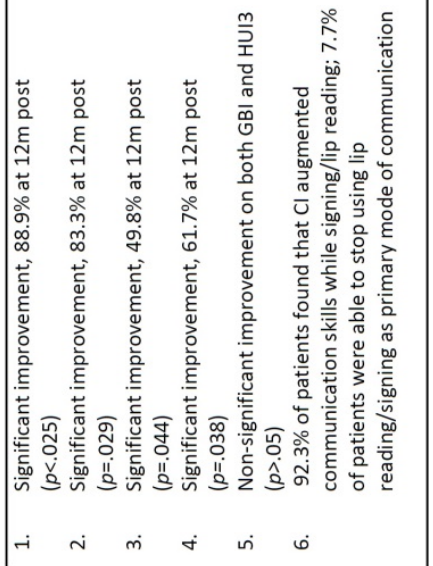 \\
\hline & 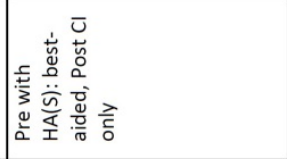 & \begin{tabular}{|l}
$\frac{5}{3}$ \\
0 \\
$\frac{5}{5}$ \\
ch
\end{tabular} & 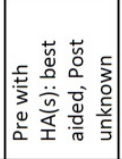 & $\begin{array}{l}\frac{5}{3} \\
0 \\
\frac{5}{5} \\
5\end{array}$ \\
\hline 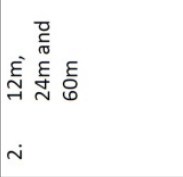 & 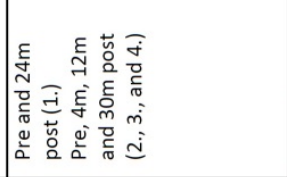 & 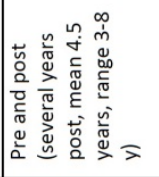 & 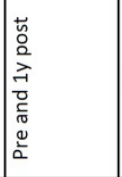 & 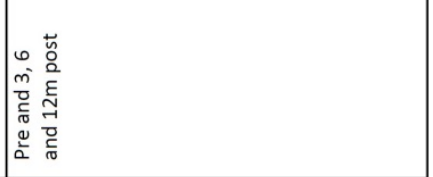 \\
\hline 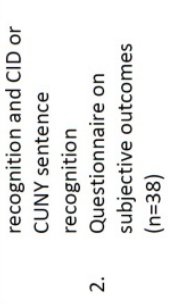 & 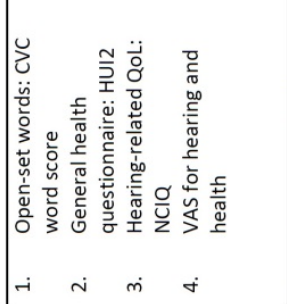 & 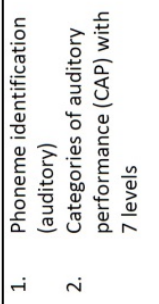 & 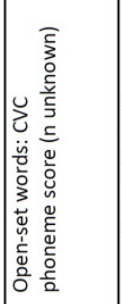 & 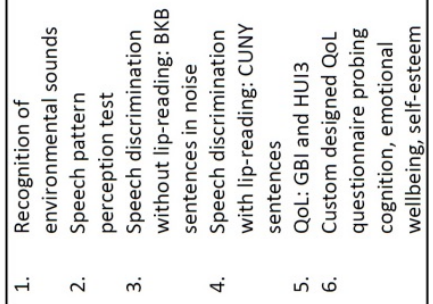 \\
\hline 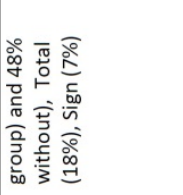 & 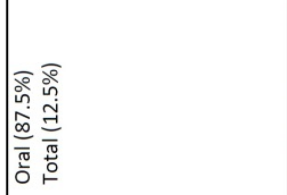 & 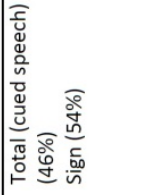 & $\begin{array}{l}\frac{c}{3} \\
\text { 童 } \\
\frac{5}{5}\end{array}$ & 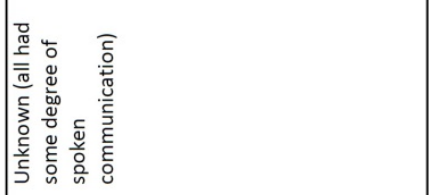 \\
\hline & 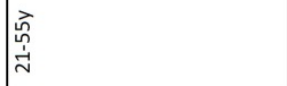 & 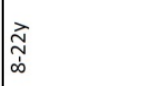 & 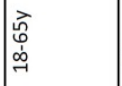 & $\underset{\substack{\vec{\wedge} \\
\vec{\wedge}}}{\overrightarrow{2}}$ \\
\hline & $\infty$ & $m$ & $\stackrel{\infty}{\infty}$ & $\stackrel{\sim}{~}$ \\
\hline & $\begin{array}{l}\dot{\pi} \\
\stackrel{0}{0} \\
\stackrel{0}{0} \hat{\circ} \\
\frac{0}{0}\end{array}$ & 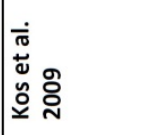 & 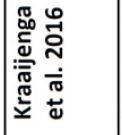 & 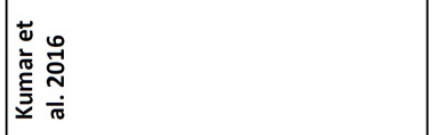 \\
\hline
\end{tabular}


Systematic review part 1: clinical effectiveness

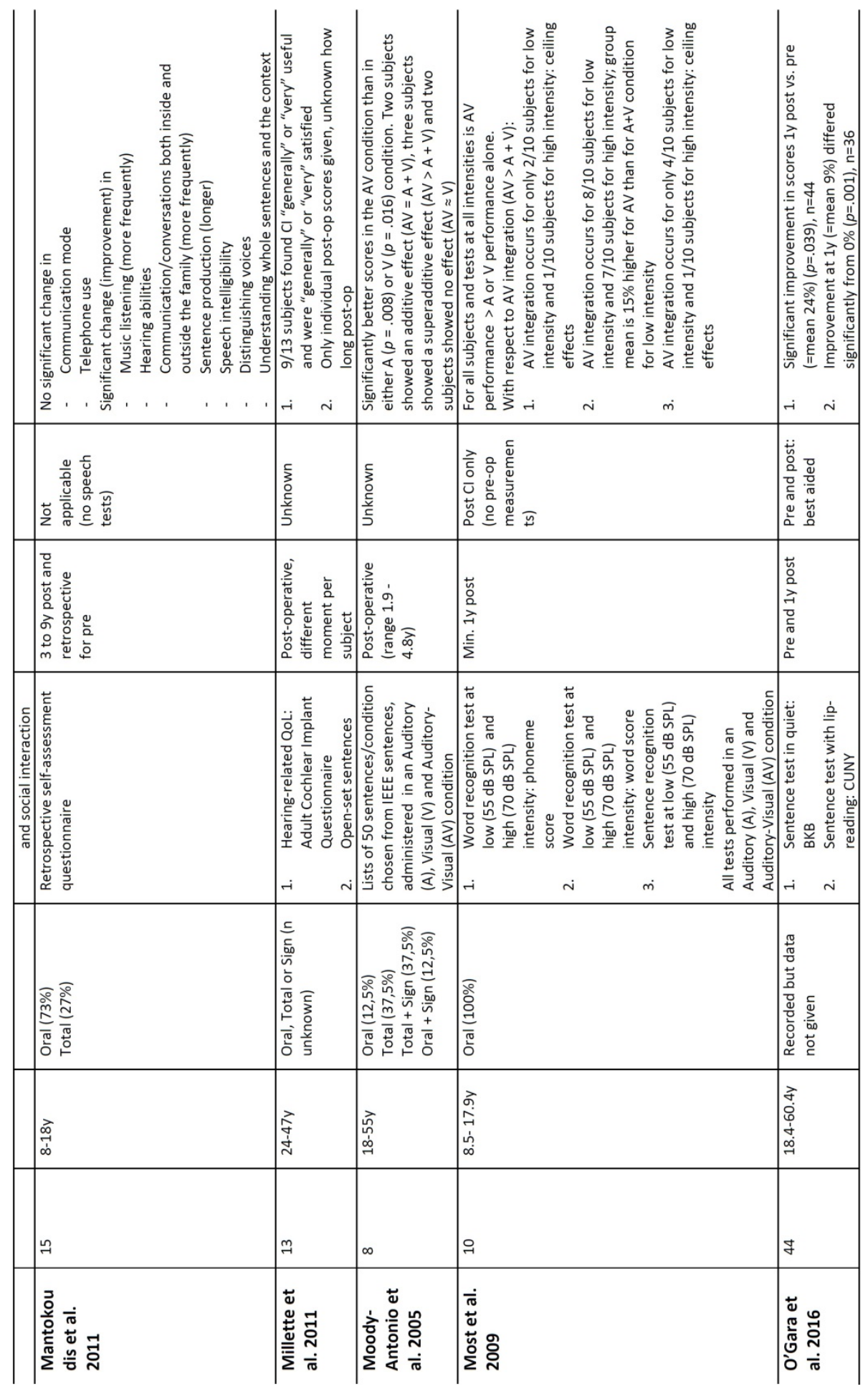


Chapter 4

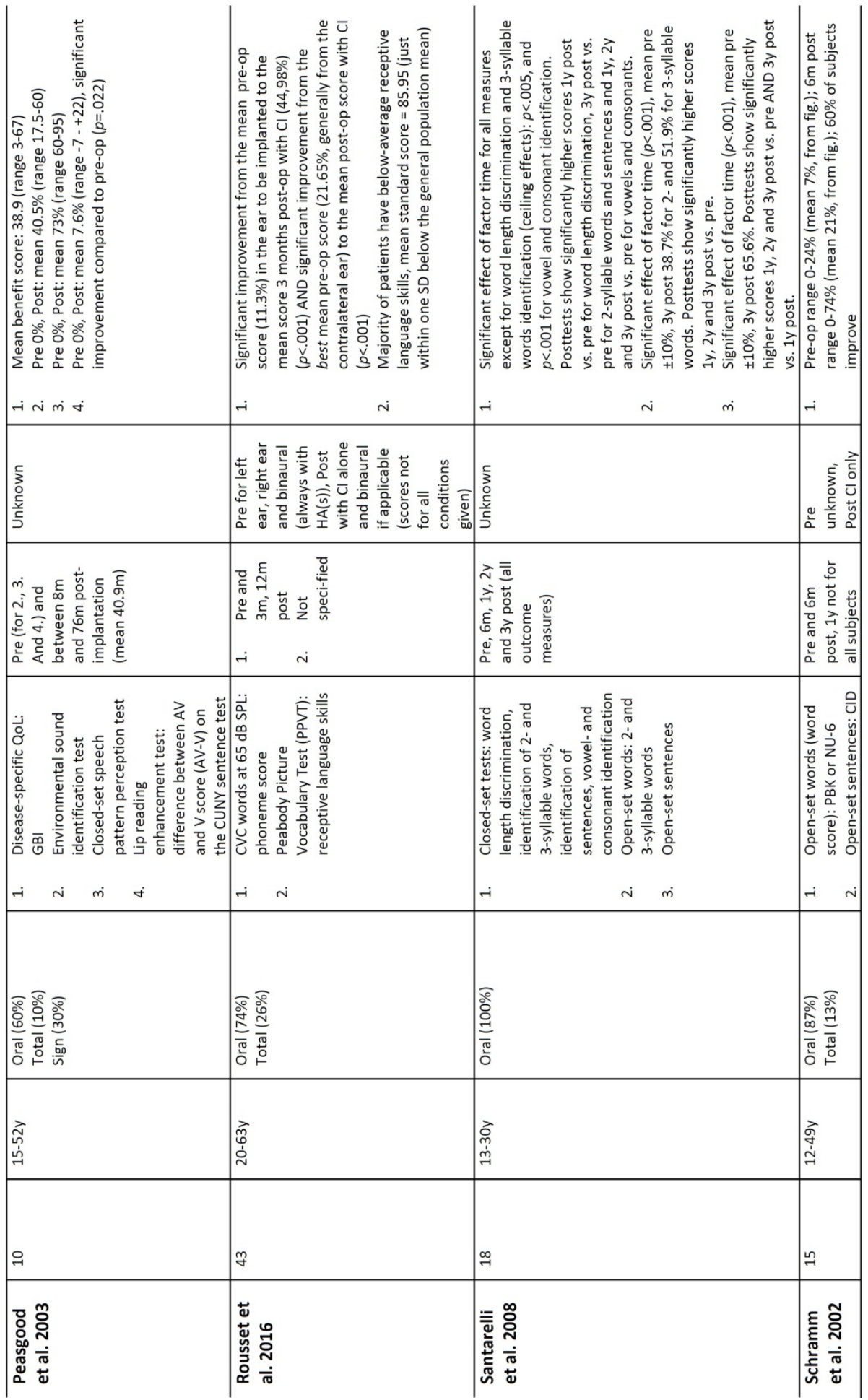


Systematic review part 1: clinical effectiveness

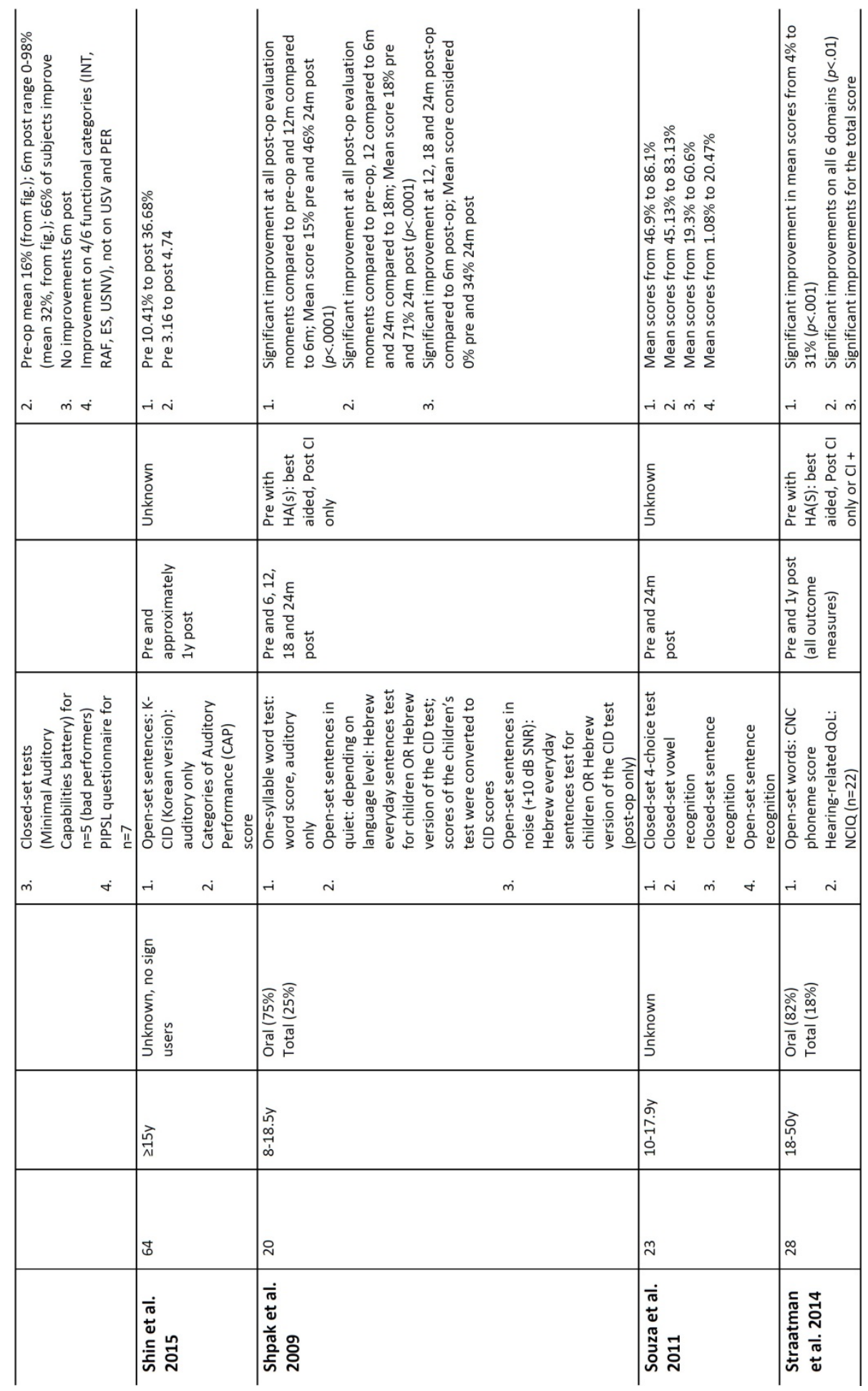


Chapter 4

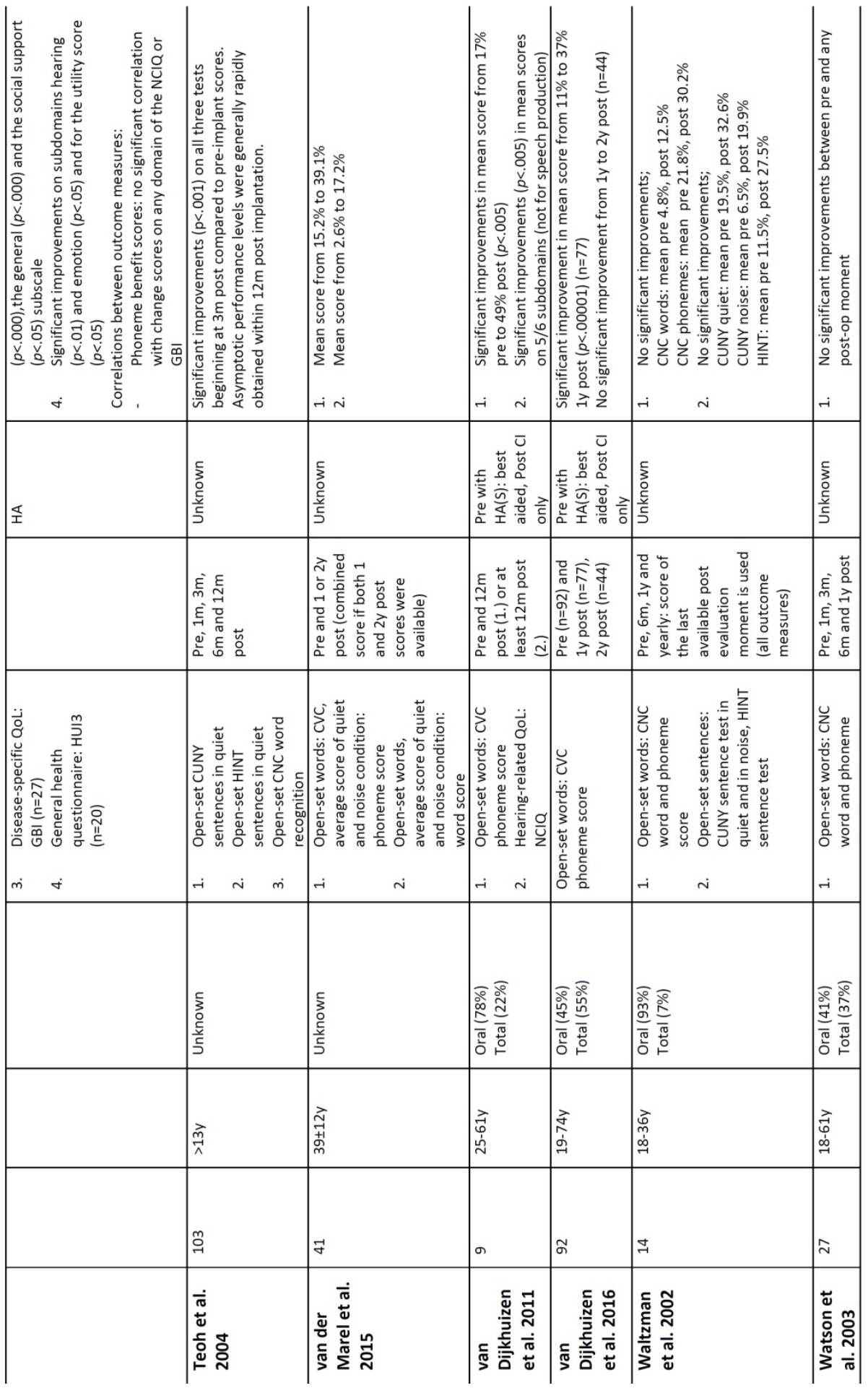


Systematic review part 1: clinical effectiveness

\begin{tabular}{|c|c|c|c|}
\hline 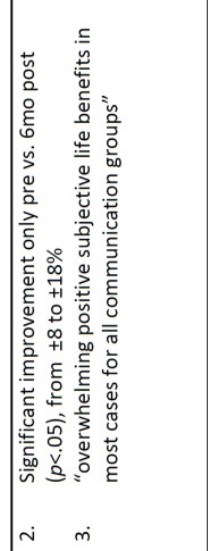 & 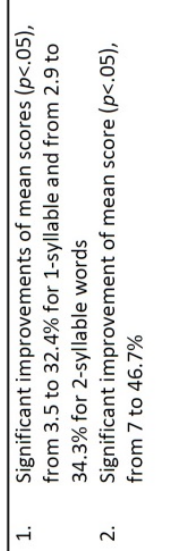 & 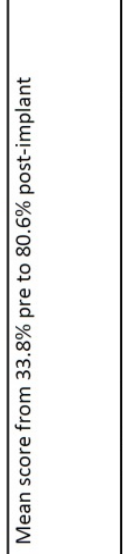 & 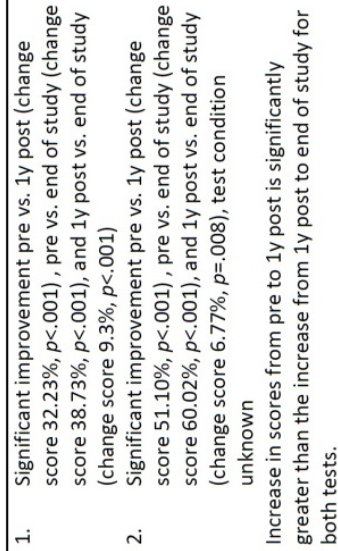 \\
\hline & \begin{tabular}{|l}
$\frac{5}{5}$ \\
0 \\
0 \\
$\frac{5}{5}$ \\
5
\end{tabular} & \begin{tabular}{|l}
$\frac{5}{3}$ \\
0 \\
$\frac{0}{5}$ \\
$\frac{5}{5}$
\end{tabular} & 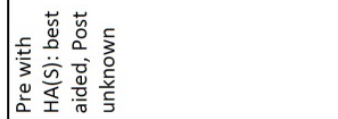 \\
\hline 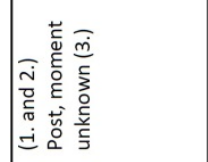 & 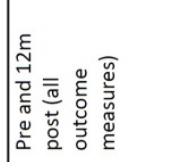 & 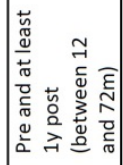 & 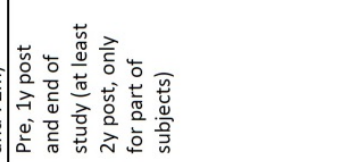 \\
\hline 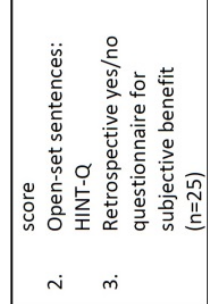 & 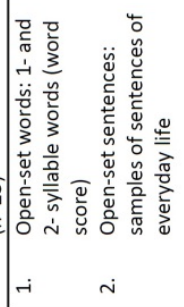 & 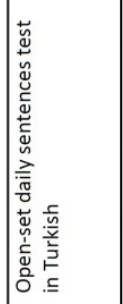 & 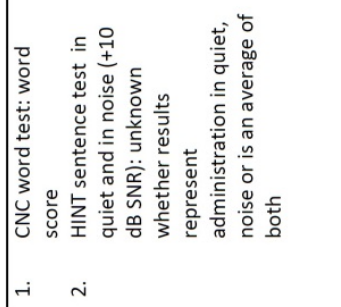 \\
\hline 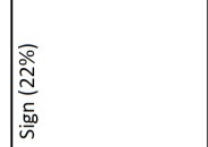 & 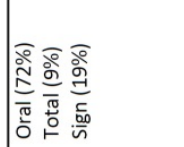 & \begin{tabular}{|l}
$\frac{\xi}{3}$ \\
0 \\
$\frac{5}{5}$ \\
$\frac{5}{5}$
\end{tabular} & 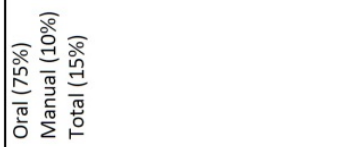 \\
\hline & 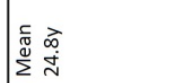 & | & 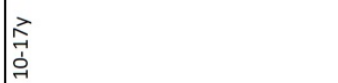 \\
\hline & $\tilde{m}$ & $\ddot{\sim}$ & 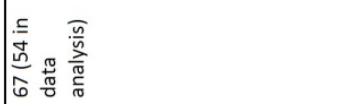 \\
\hline & 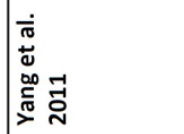 & 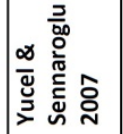 & 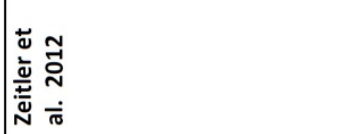 \\
\hline
\end{tabular}




\section{APPENDIX C: Guidance IHE criteria}

IHE's quality appraisal checklist for case-series studies including adapted guidance for rating the criteria (Guo et al., 2016; Moga et al., 2012)

\section{Study objective}

1. Was the hypothesis / aim / objective of the study clearly stated?

Yes: The hypothesis/aim/objective of the study was clearly reported (includes patients, intervention, and outcome).

Partial: Only one or two components (patients, intervention, or outcome) were included

No: The hypothesis/aim/objective was not reported.

Comment: Can be reported either in the abstract or introduction of the study.

Study design

\section{Was the study conducted prospectively?}

Yes: It was clearly stated that the study was conducted prospectively.

Unclear: Unclear or no information was provided.

No: The study clearly stated it was a retrospective study.

Comment: Choose 'Unclear' when it is not specifically mentioned that the study is conducted retroor prospectively or when the patient selection is retrospective but the outcome assessment prospective (e.g. patients are asked to participate).

\section{Were the cases collected in more than one center?}

Yes: Cases were collected in more than one center (multicenter study).

Unclear: Unclear where the patients came from.

No: Cases were collected from one center.

4. Were patients recruited consecutively?

Yes: There was a clear statement or it was clear from the context that the patients were recruited consecutively; or the study stated that all eligible patients were recruited.

Unclear: No information was provided about the method used to recruit patients in the study.

No: The study clearly stated that patients were not recruited consecutively; or the patients were recruited based on other criteria such as access to intervention determined by the distance or availability of resources.

Comment: In studies where patients have to give their consent to actively participate (e.g. perform additional auditory tests or complete a questionnaire) answer 'Yes' when the researchers invited all eligible patients.

\section{Study population}

\section{Were the characteristics of the patients included in the study described?}

Yes: The following characteristics should be reported: age (or age range or pre- vs. perilingual) at onset of deafness, age (or age range) at implantation, primary communication mode.

Partial: Some, but not all of the most relevant characteristics were reported.

No: Only the number of patients was reported.

Comment: instead of age at onset of deafness, age at diagnosis is also allowed.

6. Were the eligibility criteria (i.e., inclusion and exclusion criteria) for entry into the study clearly stated?

Yes: Both inclusion and exclusion criteria were reported. 
Partial: Either the inclusion or exclusion criteria were reported.

No: Neither inclusion nor exclusion criteria were reported.

Comment: mentioned criteria should be specific for the conducted study (no general "implantation criteria") and should be separate and distinguishable from the description of patient characteristics of the included patients.

7. Did patients enter the study at a similar point in the disease?

Yes: Onset of severe hearing loss/ deafness before the age of four AND age at implantation after the age of 10 for all subjects.

Unclear: There was not enough information on patients' characteristics to make a judgement.

No: Later onset of severe hearing loss/deafness OR younger age at implantation for some subjects.

Intervention and cointervention

\section{Was the intervention of interest clearly described?}

Yes: All the most relevant characteristics of the intervention were described: which implant was used, which speech coding strategy and how post-implant rehabilitation took place.

Partial: Some, but not all of the most relevant characteristics were reported.

No: it was only mentioned that cochlear implantation took place.

\section{Outcome measures}

\section{Were relevant outcome measures established a priori?}

Yes: All relevant outcome measures were stated in the introduction or methods section.

Partial: Some, but not all, of the relevant outcome measures were stated in the introduction or method section.

No: None of the relevant outcome measures were stated in the introduction or method section.

10. Were the relevant outcomes measured using appropriate objective/subjective methods?

Yes: All relevant outcomes were measured with appropriate methods. These measures include standardized clinical tests or validated questionnaires.

Partial: Some, but not all, relevant outcomes were measured with appropriate methods.

No: The methods used to measure the relevant outcomes were inappropriate.

Comment: Answer 'No' if it is unknown whether the outcome measures were appropriate (when insufficient information is given).

11. Were the relevant outcome measures made before and after the intervention?

Yes: The relevant outcome measures were made before and after intervention; or the baseline measurements were not possible.

Partial: The study did not report when the outcome measures were made.

No: The outcome measures were only made after the intervention.

Comment: Answer 'Yes' in case of retrospective questionnaires.

Statistical analysis

12. Were the statistical tests used to assess the relevant outcomes appropriate?

Yes: The statistical tests were used appropriately. Answer "yes" if no statistical analysis was performed and reasons for this were stated.

Unclear: The statistical tests were not described in the methods section of the study.

No: The statistical tests used were inappropriate.

Results and conclusions

13. Was follow-up long enough for important events and outcomes to occur?

Yes: It was clearly reported that outcomes were assessed at least 6 months post-implantation for all subjects. 


\section{Chapter 4}

Unclear: The length of the follow-up or post-operative evaluation moment was not clearly reported. No: Outcomes were assessed less than 6 months post-implantation.

\section{Were losses to follow-up reported?}

Yes: The number or proportion of patients lost to follow-up was clearly reported; the authors reported outcome results on all patients initially included; or the number lost to follow-up can be subtracted from the number of patients enrolled and the number of patients included in the final analysis.

Unclear: There was a discrepancy between the number or proportion of patients reported in tables, figures, and text.

No: The number of proportion of patients lost to follow-up was not reported.

Comment: In studies where patients have to give their consent to actively participate, patients who do not respond or who do not want to participate are considered 'losses to follow-up'.

15. Did the study provide estimates of the random variability in the data analysis of relevant outcomes?

Yes: The estimates of random variability (e.g. standard error, standard deviation, confidence interval for normally distributed data or range, and interquartile range for nonnormally distributed data) were reported for all the relevant outcomes or could be calculated from the raw data presented in the study.

Partial: The estimates of random variability were reported for some, but not all, of the relevant outcomes.

No: The estimates of the random variability were not reported for any of the relevant outcomes.

16. Were the adverse events reported?

Yes: it was mentioned whether adverse events occurred during surgery or $\mathrm{Cl}$ fitting.

Partial: It was only mentioned whether adverse events occurred with respect to surgery OR with respect to fitting, not both.

No: There was no statement about the presence or absence of adverse events.

17. Were the conclusions of the study supported by results?

Yes: The conclusions of the study were supported by the evidence presented in the results and discussion sections.

Unclear: Unclear conclusion statement that makes it difficult to link the presented evidence to conclusions.

No: The conclusions were not supported by the evidence presented in the results and discussion sections.

Comment: Answer 'No' also when a part of the conclusion statement is not supported by the data.

Competing interest and source of support

18. Were both competing interests and sources of support for the study reported?

Yes: Both competing interests and sources of support (financial or other) received for the study were reported; or the absence of any competing interests and source of support was acknowledged.

Partial: Either the competing interest or source of support was reported.

No: Neither competing interests nor sources of support were reported. 
APPENDIX D: Results IHE Quality tool

1. Objective clearly stated?

\section{Author}

Arisi et al. 2010

Bassim et al. 2005

Bellelli et al. 2016

Bosco et al. 2013

Caposecco et al. 2012

Chee et al. 2004

Craddock et al. 2016

Cusumano et al. 2017

Derinsu et al. 2002

Duchesne et al. 2017

Heywood et al. 2016

Kaplan et al. 2003

Klop et al. 2007

Kos et al. 2009

Kraaijenga et al. 2015

Kumar et al. 2016

Mantokoudis et al. 2011

Millette et al. 2011

Moody-Antonio et al. 2005

Most et al. 2009

O'Gara et al. 2016

Peasgood et al. 2003

Rousset et al. 2016

Santarelli et al. 2008

Schramm et al. 2002

Shin et al. 2015

Shpak et al. 2009

Souza et al. 2011

Straatman et al. 2014

Teoh et al. 2004

Van der Marel et al. 2015

Van Dijkhuizen et al. 2011

Van Dijkhuizen et al. 2016

Waltzman et al. 2002

Watson et al. 2003

Yang et al. 2011

Yucel \& Sennaroglu 2007

Zeitler et al. 2012

\section{Prospective study?}

\section{Multicentre}

study?

Yes Yes No

Yes No No

Yes Unclear No

Yes Unclear No

Yes No No

Yes Unclear (abstract: prospective No

design, but patient selection is retrospective)

\begin{tabular}{|c|c|c|}
\hline $\begin{array}{l}\text { Partial (aim vaguely } \\
\text { described) }\end{array}$ & No & No \\
\hline Yes & No & No \\
\hline $\begin{array}{c}\text { Yes (in abstract + } \\
\text { introduction) }\end{array}$ & Unclear & No \\
\hline Yes & Unclear & Unclear \\
\hline Yes & No & No \\
\hline Yes & No & No \\
\hline Yes & Unclear & No \\
\hline Yes & Unclear & No \\
\hline Yes & No & No \\
\hline Yes & No & No \\
\hline Yes & No & No \\
\hline Yes & No & No \\
\hline Yes & Unclear & No \\
\hline Yes & Unclear & Unclear \\
\hline Yes & No & No \\
\hline Yes & Unclear & Unclear \\
\hline Yes & Unclear & No \\
\hline Yes & Unclear & No \\
\hline Yes & No & No \\
\hline Yes & Yes & No \\
\hline Yes & Unclear & No \\
\hline Yes & Yes & No \\
\hline Yes & Yes & No \\
\hline Yes & Unclear & Unclear \\
\hline Yes & Unclear & No \\
\hline Yes & Yes & No \\
\hline Yes & Yes & No \\
\hline Yes & Yes & Unclear \\
\hline $\begin{array}{c}\text { Partial (no clear } \\
\text { outcome measure) }\end{array}$ & Unclear & Unclear \\
\hline $\begin{array}{l}\text { Partial (vague outcome } \\
\text { measure) }\end{array}$ & Unclear & No \\
\hline Yes & Unclear & No \\
\hline Partial (no clear & No & No \\
\hline
\end{tabular}




\section{Chapter 4}

4. Patients recruited

Author

Arisi et al. 2010

Bassim et al. 2005

Bellelli et al. 2016

Bosco et al. 2013

Caposecco et al. 2012

Chee et al. 2004

Craddock et al. 2016

Cusumano et al. 2017

Derinsu et al. 2002

Duchesne et al. 2017

Heywood et al. 2016

Kaplan et al. 2003

Klop et al. 2007

Kos et al. 2009

Kraaijenga et al. 2015

Kumar et al. 2016

Mantokoudis et al. 2011

Millette et al. 2011

Moody-Antonio et al. 2005

Most et al. 2009

O'Gara et al. 2016

Peasgood et al. 2003

Rousset et al. 2016

Santarelli et al. 2008

Schramm et al. 2002

Shin et al. 2015

Shpak et al. 2009

Souza et al. 2011

Straatman et al. 2014

Teoh et al. 2004

Van der Marel et al.

2015

Van Dijkhuizen et al.

2011

Van Dijkhuizen et al.

2016

Waltzman et al. 2002

Watson et al. 2003

Yang et al. 2011

Yucel \& Sennaroglu

2007

Zeitler et al. 2012

Unclear

Unclear

Yes

Unclear invited)

Yes

Yes

Unclear

Unclear

Unclear

Yes

Unclear

Unclear

Unclear

Unclear participated) invited) Unclear Yes Yes

Yes

Yes

Yes

Unclear

Unclear

Unclear

Yes

Unclear

Unclear

Unclear

Unclear

Unclear

Yes

Yes consecutively?

Yes (clearly mentioned how many

patients met inclusion criteria)

Yes (all eligible patients were

Yes (clear from context)

No (not all eligible patients

Yes (all eligible patients were

5. Patient characteristics described?

Yes

Partial (no specific age at onset or age at implantation given)

Yes

Yes

Partial (age at implantation and communication mode not given)

Yes

Partial (communication mode not given) Yes

Partial (no specific age at onset given)

Yes

Yes

Yes

Yes

Partial (communication mode not given)

No

Yes

Partial (age at onset and communication mode not given)

Partial (no specific age at onset given)

\section{Yes}

Partial (no details on communication mode) Yes

Partial (no specific age at onset given)

Yes

Yes

No (only number of patients reported) Yes

Partial (age at onset and communication mode not given) Yes

No (only number of patients reported)

Partial (communication mode not given)

No (patients recruited based on specific criterion)

No (patients recruited based on specific criterion)

Yes

Yes

Yes

Partial (age at implantation not given)

Partial (no specific age at onset or age at implantation given)

Partial (communication mode not given)

Partial (no specific age at onset given) 
6. Eligibility criteria clearly

Author

Arisi et al. 2010

Bassim et al. 2005

Bellelli et al. 2016

Bosco et al. 2013

Caposecco et al. 2012

Chee et al. 2004

Craddock et al. 2016

Cusumano et al. 2017

Derinsu et al. 2002

Duchesne et al. 2017

Heywood et al. 2016

Kaplan et al. 2003

Klop et al. 2007

Kos et al. 2009

Kraaijenga et al. 2015

Kumar et al. 2016

Mantokoudis et al. 2011

Millette et al. 2011

Moody-Antonio et al.

2005

Most et al. 2009

O'Gara et al. 2016

Peasgood et al. 2003

Rousset et al. 2016

Santarelli et al. 2008

Schramm et al. 2002

Shin et al. 2015

Shpak et al. 2009

Souza et al. 2011

Straatman et al. 2014

Teoh et al. 2004

Van der Marel et al.

2015

Van Dijkhuizen et al.

2011

Van Dijkhuizen et al.

2016

Waltzman et al. 2002

Watson et al. 2003

Yang et al. 2011

Yucel \& Sennaroglu 2007

Zeitler et al. 2012

stated?

Yes

Partial (no exclusion criteria)

No

Yes

Partial (no exclusion criteria)

No

No

Yes

No

Partial

Partial

Partial (no exclusion criteria)

Yes

No

Partial

Partial (only exclusion criteria)

Partial (no exclusion criteria) Partial

Partial (no exclusion criteria)

$$
\text { No }
$$

Yes

No

No

Yes

Partial (no exclusion criteria)

Partial (no clear description of

$$
\text { No }
$$

Partial (no exclusion criteria; are inclusion criteria)

criteria are inclusion criteria or patient characteristics)

Partial (no exclusion criteria)

Yes

Yes

Partial (unclear if reported

criteria are exclusion criteria or

patient characteristics)

No

Yes either in- and exclusion criteria) unclear whether reported criteria

Partial (unclear whether reported

\section{Study entry at similar point in}

disease?

Yes

No (onset $>4$ years for some patients)

Unclear

Yes

No (progressive hearing loss in some patients)

No (onset of deafness before the age of six)

No (onset of deafness before the age of seven)

Yes

Yes

Yes

No (onset of deafness before the age of six)

No (onset of deafness before the age of six) Yes

No (implantation $<10 y$ for some patients)

Yes

Yes

No (implantation $<10 y$ for some patients)

Yes

Yes

No (implantation $<10 y$ for some patients)

Yes

No (onset $>4$ years or progressive hearing loss in some patients)

Unclear (onset unclear: text contradicts table)

Yes

Yes

Unclear (not enough information on age at onset)

No (implantation $<10$ y for some patients)

Unclear (not enough information on age at onset)

Yes

Yes Yes

Yes

No Yes

No Yes

Yes

No

Yes 


\section{Chapter 4}

Author

Arisi et al. 2010

Bassim et al. 2005

Bellelli et al. 2016

Bosco et al. 2013

Caposecco et al. 2012

Chee et al. 2004

Craddock et al. 2016

Cusumano et al. 2017

Derinsu et al. 2002

Duchesne et al. 2017

Heywood et al. 2016

Kaplan et al. 2003

Klop et al. 2007

Kos et al. 2009

Kraaijenga et al. 2015

Kumar et al. 2016

Mantokoudis et al. 2011

Millette et al. 2011

Moody-Antonio et al.

2005

Most et al. 2009

O'Gara et al. 2016

Peasgood et al. 2003

Rousset et al. 2016

Santarelli et al. 2008

Schramm et al. 2002

Shin et al. 2015

Shpak et al. 2009

Souza et al. 2011

Straatman et al. 2014

Teoh et al. 2004

Van der Marel et al.

2015

Van Dijkhuizen et al.

2011

Van Dijkhuizen et al.

2016

Waltzman et al. 2002

Watson et al. 2003

Yang et al. 2011

Yucel \& Sennaroglu

2007

Zeitler et al. 2012
8. Intervention clearly described?

9. Outcome measures

established a priori?

Partial (no info on post-implant rehabilitation) Yes No

Partial (no info on post-implant rehabilitation)

Partial (no info on speech coding strategy)

No

Partial (no info on speech coding strategy or post-implant rehabilitation)

No

Yes

Yes

Yes

Yes

Yes

Yes

Partial (no info on post-implant rehabilitation)

Yes

Partial (no info on speech coding strategy) No

Partial (no info on post-implant rehabilitation)

Partial (no info on speech coding strategy)

Partial (no info on speech coding strategy or post-implant rehabilitation)

No Yes

No

Yes

Partial (no info on speech coding strategy or Yes post-implant rehabilitation)

Yes

Yes

Partial (no info on post-implant rehabilitation)

Yes

Partial (no info on post-implant rehabilitation) No

Partial (no info on speech coding strategy or post-implant rehabilitation)

Partial (no info on post-implant rehabilitation) Yes Yes

Partial (no info on speech coding strategy or post-implant rehabilitation)

No

Yes

Yes

Yes

Yes

Yes

Yes

Partial (no info on post-implant rehabilitation) Yes

Partial (no info on speech coding strategy) Yes

No

Yes

Partial (no info on post-implant rehabilitation)

Partial (no info on speech coding strategy)

No (only in results section)

Yes $\quad$ Yes

Partial (no info on speech coding strategy)

Yes

Partial (no info on post-implant rehabilitation) No

Partial (no info on post-implant rehabilitation) Partial (no info on implant type or postimplant rehabilitation)

No 


\section{Outcomes measured with}

Author

Arisi et al. 2010

Bassim et al. 2005

Bellelli et al. 2016

Bosco et al. 2013

Caposecco et al. 2012

Chee et al. 2004

Craddock et al. 2016

Cusumano et al. 2017

Derinsu et al. 2002

Duchesne et al. 2017

Heywood et al. 2016

Kaplan et al. 2003

Klop et al. 2007

Kos et al. 2009

Kraaijenga et al. 2015

Kumar et al. 2016

Mantokoudis et al. 2011

Millette et al. 2011

Moody-Antonio et al.

2005

Most et al. 2009

O'Gara et al. 2016

Peasgood et al. 2003

Rousset et al. 2016

Santarelli et al. 2008

Schramm et al. 2002

Shin et al. 2015

Shpak et al. 2009

Souza et al. 2011

Straatman et al. 2014

Teoh et al. 2004

Van der Marel et al.

2015

Van Dijkhuizen et al.

2011

Van Dijkhuizen et al.

2016

Waltzman et al. 2002

Watson et al. 2003

Yang et al. 2011

Yucel \& Sennaroglu

2007

Zeitler et al. 2012

appropriate methods?

\section{Outcomes measured before and after the intervention?}

\begin{tabular}{|c|c|}
\hline No (unknown whether tests are validated) & Yes \\
\hline Yes & $\begin{array}{l}\text { Partial ( } n \text { varies between tests } \\
\text { and evaluation moments) }\end{array}$ \\
\hline Yes & Yes \\
\hline Partial (interview not validated) & Yes \\
\hline Partial (study survey not validated) & Yes \\
\hline No (questionnaire not validated) & Yes \\
\hline Yes & Yes \\
\hline Yes & Yes \\
\hline No (live presentation) & Yes \\
\hline Partial (questionnaire not validated) & Yes \\
\hline Partial (questionnaire not validated) & Yes \\
\hline Partial (questionnaire not validated) & $\begin{array}{l}\text { Partial (no pre-op score for } \\
\text { speech test) }\end{array}$ \\
\hline Yes & Yes \\
\hline Yes & Yes \\
\hline Yes & Yes \\
\hline $\begin{array}{c}\text { Partial (not all outcome measures are } \\
\text { standardised tests, BKB unclear if quiet or } \\
\text { noise) }\end{array}$ & Yes \\
\hline No (questionnaire not validated) & Yes \\
\hline Partial (questionnaire not validated) & $\begin{array}{l}\text { Partial (no pre-op score for } \\
\text { speech test) }\end{array}$ \\
\hline Yes & No \\
\hline Yes & No \\
\hline Yes & Yes \\
\hline Yes & Yes \\
\hline Yes & Yes \\
\hline Yes & Yes \\
\hline Yes & Yes \\
\hline Yes & Yes \\
\hline Yes & Yes \\
\hline Yes & Yes \\
\hline Yes & Yes \\
\hline Yes & Yes \\
\hline $\begin{array}{l}\text { Partial (average of different test conditions, } \\
\text { not clearly stated) }\end{array}$ & Yes \\
\hline Yes & Yes \\
\hline Yes & Yes \\
\hline Partial (HINT condition not defined) & Yes \\
\hline Partial (questionnaire not validated) & Yes \\
\hline No (unknown whether tests are validated) & Yes \\
\hline $\begin{array}{c}\text { No (unknown whether sentence test is } \\
\text { validated) }\end{array}$ & Yes \\
\hline Partial (HINT unclear whether quiet or noise) & Yes \\
\hline
\end{tabular}




\section{Chapter 4}

Author

Arisi et al. 2010

Bassim et al. 2005

Bellelli et al. 2016

Bosco et al. 2013

Caposecco et al. 2012

Chee et al. 2004

Craddock et al. 2016

Cusumano et al. 2017

Derinsu et al. 2002

Duchesne et al. 2017

Heywood et al. 2016

Kaplan et al. 2003

Klop et al. 2007

Kos et al. 2009

Kraaijenga et al. 2015

Kumar et al. 2016

Mantokoudis et al. 2011

Millette et al. 2011

Moody-Antonio et al. 2005

Most et al. 2009

O'Gara et al. 2016

Peasgood et al. 2003

Rousset et al. 2016

Santarelli et al. 2008

Schramm et al. 2002

Shin et al. 2015

Shpak et al. 2009

Souza et al. 2011

Straatman et al. 2014

Teoh et al. 2004

Van der Marel et al.

2015

Van Dijkhuizen et al.

2011

Van Dijkhuizen et al.

2016

Waltzman et al. 2002

Watson et al. 2003

Yang et al. 2011

Yucel \& Sennaroglu

2007

Zeitler et al. 2012

\section{Statistical tests appropriate?}

No (no statistical tests performed, insufficient descriptive analysis, varying group

$$
\text { size) }
$$

Yes

Yes for comparison pre- and post-op scores

(Unclear for comparison pre- vs. perilingual onset)

Yes

Yes

No (no description in methods, paired t-tests without correction for multiple testing + unknown whether difference scores normally distributed)

Unclear (unclear whether test assumptions were met, unclear presentation of results)

No (no statistical tests performed, insufficient descriptive analysis)

Yes but mean pre-op for $n=21$, post-op for $n=19$

Yes

Unclear (test used for comparison of composite scores not mentioned)

No (paired t-test for $n=8$ without correction for multiple testing + unknown whether difference scores were normally distributed)

Unclear (insufficient information on tests used)

NA (no comparison of pre- and post-op scores)

Unclear (test not mentioned for pre-post comparisons)

Yes

No (pre-requisites for chi-square test not met)

Yes

Yes

Unclear (insufficient information on tests used)

Yes

Yes

Yes for comparison pre- and post-op scores

No for tests assessing continued increase over time (pairwise comparisons between evaluation moments too limited)

No (descriptive analysis is appropriate but insufficient given the data)

No (performed analysis insufficient to answer questions on prelingual patients as a group)

Yes

No (adequate description of the tests but no results given) Yes

No (paired samples t-test for pre-post comparisons without correction for multiple testing; comparison between brands based on groups containing different patients) NA (no comparison of pre- and post-op scores)

No (paired t-test for $n=9+$ unknown whether difference scores were normally distributed)

Yes

Yes

Unclear (tests not mentioned)

Unclear (type of t-test not mentioned)

No (correlation used for pre-post comparisons)

Yes 


\section{Follow-up long}

Author

Arisi et al. 2010

Bassim et al. 2005

Bellelli et al. 2016

Bosco et al. 2013

Caposecco et al. 2012

Chee et al. 2004

Craddock et al. 2016

Cusumano et al. 2017

Derinsu et al. 2002

Duchesne et al. 2017

Heywood et al. 2016

Kaplan et al. 2003

Klop et al. 2007

Kos et al. 2009

Kraaijenga et al. 2015

Kumar et al. 2016

Mantokoudis et al. 2011

Millette et al. 2011

Moody-Antonio et al. 2005

Most et al. 2009

O'Gara et al. 2016

Peasgood et al. 2003

Rousset et al. 2016

Santarelli et al. 2008

Schramm et al. 2002

Shin et al. 2015

Shpak et al. 2009

Souza et al. 2011

Straatman et al. 2014

Teoh et al. 2004

Van der Marel et al.

2015

Van Dijkhuizen et al.

2011

Van Dijkhuizen et al.

2016

Waltzman et al. 2002

Watson et al. 2003

Yang et al. 2011

Yucel \& Sennaroglu

2007

Zeitler et al. 2012

enough?

Yes many patients $>6 \mathrm{~m}$ )

Unclear (not reported)

Yes

Yes

Yes

Yes

Yes

Yes

Yes

Yes

Yes

Yes

Yes not always given)

Yes

\section{Losses to follow-up reported?}

Yes

Unclear (unknown for how Unclear (discrepancy between text and Table 3)

No (<6 m for some patients)

Unclear (evaluation moment

Yes Yes

Yes Unclear (discrepancy between $n^{0}$ of patients

meeting criteria and $n^{0}$ of patients with data)

Unclear (vague, additional exclusion afterwards)

Yes Yes

Yes Yes

Yes Yes

No $(<6 \mathrm{~m}$ for some patients $\quad$ Yes

and not clearly reported)

Yes Yes

Yes Yes

Yes No

Yes Yes

Yes Yes

Yes Yes

Yes Unclear (discrepancy between figures and text)

Yes Yes

Yes $\quad$ Yes

Yes $\quad$ Yes

Yes Yes

Yes Yes

Yes Unclear (no statement, deduction from figures uncertain)

Yes Yes

Yes Yes 


\section{Chapter 4}

\section{Estimates of random}

Author

Arisi et al. 2010

Bassim et al. 2005

Bellelli et al. 2016

Bosco et al. 2013

Caposecco et al. 2012

Chee et al. 2004

Craddock et al. 2016

Cusumano et al. 2017

Derinsu et al. 2002

Duchesne et al. 2017

Heywood et al. 2016

Kaplan et al. 2003

Klop et al. 2007

Kos et al. 2009

Kraaijenga et al. 2015

Kumar et al. 2016

Mantokoudis et al. 2011

Millette et al. 2011

Moody-Antonio et al. 2005

Most et al. 2009

O'Gara et al. 2016

Peasgood et al. 2003

Rousset et al. 2016

Santarelli et al. 2008

Schramm et al. 2002

Shin et al. 2015

Shpak et al. 2009

Souza et al. 2011

Straatman et al. 2014

Teoh et al. 2004

Van der Marel et al.

2015

Van Dijkhuizen et al.

2011

Van Dijkhuizen et al.

2016

Waltzman et al. 2002

Watson et al. 2003

Yang et al. 2011

Yucel \& Sennaroglu

2007

Zeitler et al. 2012 variability?

16. Adverse events reported?

Yes

No

Yes

Yes

Yes (raw data given)

Yes (raw data given)

Yes

Partial

Yes (raw data given)

Yes (raw data given) Yes

No

Partial

Yes (raw data in figures)

Partial (discrepancy between

figure and text)

$$
\text { Partial }
$$

No

Yes

Partial (only surgical)

No

No

Yes

No

Partial (only for fitting)

Partial

No

Yes

No

Partial (only surgical)

Partial (only surgical)

Partial (only surgical)

No (not for prelingual group)

Yes (raw data in figures)

Yes (raw data given)

No

No

No

No

Yes No

Yes Partial

Yes (raw data in table)

Yes

Yes

Partial (only surgical)

No

No

Yes (raw data in figures) Yes

No No

Yes (raw data in Apdx)

Yes (raw data given)

Partial (not for speech tests)

Yes

Yes

No

No

No

No

Partial (only surgical)

Yes (raw data in figure)

Yes

Yes

Yes

Yes (raw data in figures)

No

Partial (unclear for fitting)

No

Partial (unclear what estimates

No

represent)

Yes (raw data in figure)

No

Yes

No 
18. Competing

interests \&

sources of

Author

17. Conclusions supported by results?

support?

Arisi et al. 2010
Bassim et al. 2005
Bellelli et al. 2016
Bosco et al. 2013
Caposecco et al. 2012
Chee et al. 2004
Craddock et al. 2016
Cusumano et al. 2017

Derinsu et al. 2002

Duchesne et al. 2017

Heywood et al. 2016

Kaplan et al. 2003

Klop et al. 2007

Kos et al. 2009

Kraaijenga et al. 2015

Kumar et al. 2016

Mantokoudis et al. 2011

Millette et al. 2011

Moody-Antonio et al.

2005

Most et al. 2009

O'Gara et al. 2016

Peasgood et al. 2003

Rousset et al. 2016

Santarelli et al. 2008

Schramm et al. 2002

Shin et al. 2015

Shpak et al. 2009

Souza et al. 2011

Straatman et al. 2014

Teoh et al. 2004

Van der Marel et al.

2015

Van Dijkhuizen et al.

2011

Van Dijkhuizen et al.

2016

Waltzman et al. 2002

Watson et al. 2003

Yang et al. 2011

Yucel \& Sennaroglu 2007

Zeitler et al. 2012
No (second conclusion not supported by the data)

No (statements in results and discussion sections about

benefit and plateau performance not supported by results)

Yes

Unclear (part of the conclusion not supported by the data) Yes

Unclear (broad conclusion statements, difficult to be linked) Yes

Unclear (conclusion cannot be confirmed due to

Yes

Partial

discrepancies between text and figures)

Yes Yes

Yes Yes

Yes No

Yes Partial

Yes No

NA (no conclusion statement regarding non-prognostic part Yes of the study)

No (not all conclusions supported by the data)

Yes

Unclear (not all conclusions can be linked to the data) Yes

Yes

Partial

No

Yes

Yes

No

NA (no conclusion statement regarding non-prognostic part of the study)

Yes

Partial

$$
\text { Yes }
$$

No

Partial

No (the performed statistical analysis was not sufficient to

No

support the conclusion that results continued to increase until 3 years post-op)

No (conclusions not supported by the data) No

No (conclusion that both groups performed better cannot be Yes supported by the performed analysis)

$\begin{array}{lc}\text { Yes } & \text { Partial } \\ \text { No } & \text { No } \\ \text { Yes } & \text { Partial }\end{array}$

Unclear (conclusions relate to the outcomes of other studies Partial

as well as to the newly presented data, which makes it

difficult to link the presented evidence to the conclusions)

NA (no conclusion statement regarding the non-prognostic outcomes)

NA (no conclusion statement regarding the non-prognostic outcomes)

NA (no conclusion statement regarding the non-prognostic Yes outcomes)

No (conclusions not supported by the data from the adults) Partial Yes

NA (no conclusion statement regarding the non-prognostic outcomes)

No (wrong conclusion based on wrong statistical test) No

Yes Partial

No (unclear statements that cannot be directly linked) No 

Chapter 5

\title{
Systematic review on late cochlear implantation in early deafened adults and adolescents. Part 2: predictors of performance
}

\author{
Under review \\ Joke Debruyne \\ Miranda Janssen \\ Jan Brokx
}




\section{ABSTRACT}

Objectives: Early-deafened, late-implanted adolescents and adults constitute a unique group of cochlear implant $(\mathrm{Cl})$ users, showing a large variability in outcomes. The current systematic review aimed to determine which preimplantation factors are relevant in predicting postoperative outcomes in this patient group.

Design: A systematic search for studies published between 2000 and September 2017 was performed in 5 electronic databases (PubMed, Embase, the Cochrane library, CINAHL, and Psyclnfo). Included were prognostic studies that assessed the relation between patientrelated factors and $\mathrm{Cl}$ outcomes in early-deafened but late-implanted adolescent and adult $\mathrm{Cl}$ users. Study quality was assessed with the Quality In Prognosis Studies (QUIPS) tool.

Results: The systematic search and subsequent full-text evaluation identified 21 studies examining one or more prognostic factors, 13 of which had a clear prognostic study goal. Eight out of these 13 studies had a high risk of bias for at least 1 of the 5 QUIPS domains. Analysis of the outcomes identified that communication mode (in childhood), preoperative speech intelligibility, and preoperative speech recognition scores were significantly related to speech perception outcomes for this group of $\mathrm{Cl}$ users. A number of additional factors considered worth further investigation, were also identified.

Conclusions: The analysis of the studies assessing the effect of a number of patientrelated factors on outcome with $\mathrm{Cl}$ in early-deafened adolescents and adults identified 3 potential prognostic factors and a number of additional factors to be incorporated in future research. More high-quality prognosis studies in the investigated patient population are still needed. 


\section{INTRODUCTION}

Ever since cochlear implantation ( $\mathrm{Cl}$ ) was introduced as a medical intervention to restore sensorineural hearing loss, part of the implanted population consisted of adults who had been deaf or severely hearing impaired since early childhood, or even since birth (Eisenberg, 1982). It also became clear early on that the $\mathrm{Cl}$ performance achieved by these patients was well below the performance seen in many patients with a later onset of deafness, i.e. the postlingually deaf adults (Snik et al., 1997; Waltzman et al., 1992). In part 1 of this systematic review, which focused on the clinical effectiveness of late cochlear implantation in early-deafened adults and adolescents, we did identify significant postoperative improvements of closed-set, audiovisual and open-set speech understanding in the majority of studies, published from 2000 onwards. However, as results were based on the average performance of the included subjects, this does not imply that improvements can be found in every individual patient. In fact, the variability in outcomes obtained by early-deafened $\mathrm{Cl}$ users turned out to be very large. This might be related to the large variability in the early-deafened population itself: there will be oral communicators that have consistently used their residual hearing throughout their lives, as well as sign users that have little or no experience with hearing aids and therefore auditory stimulation. It is known that when auditory deprivation is present at birth or originates in early childhood, it prevents normal maturation of the auditory system. This process cannot be reversed, even when auditory stimulation is restored later in life through a cochlear implant (Kral et al., 2017). This has as a result that early-deafened adults constitute a unique group of cochlear implant users. The factors that affect their performance are therefore not necessarily the same as those affecting the performance of postlingually deafened adults. Given the large diversity in outcomes between candidates and the uniqueness of the patient group, it is important to investigate whether, and which, patient-related factors contribute to the outcomes obtained by early-deafened, adult $\mathrm{Cl}$ users.

In recent years, a number of large scale prognosis studies have been conducted in postlingually deafened $\mathrm{Cl}$ users. Factors that were found to be related to better $\mathrm{Cl}$ outcomes in this population were a shorter duration of moderate to profound hearing loss prior to implantation (Blamey et al., 2013; Holden et al., 2013; Roditi et al., 2009), the use of hearing aids preoperatively (Holden et al., 2013; Lazard, Vincent, et al., 2012), more preoperative residual hearing (Lazard, Vincent, et al., 2012) and better preoperative speech recognition (Roditi et al., 2009). Also found to be of importance were the position of the electrode in the cochlea (Holden et al., 2013), and the age at implantation, with more negative outcomes for older patients (Blamey et al., 2013; Holden et al., 2013). Nonetheless, combining these mainly demographical prognostic factors did leave a very large part of the variation in speech recognition outcomes unexplained (Blamey et al., 2013; Lazard, Vincent, et al., 2012). The focus of a number of recent studies has therefore shifted towards exploring the influence of underlying cognitive factors; Kaandorp, Smits, 
Merkus, Festen, and Goverts (2017) for instance demonstrated that a poor verbal working-memory capacity was related to poorer speech recognition performance in a group of 24 postlingually deafened $\mathrm{Cl}$ users. This fits into the hypothesis that the unknown variability in $\mathrm{Cl}$ outcomes is determined by the subjects' ability to perform the underlying information processing tasks required to perceive spoken language (Pisoni et al., 2017).

In contrast with those recent, often large scale studies in postlingually deafened $\mathrm{Cl}$ users, most prognosis studies in our patient group of interest typically have relatively small sample sizes, and additionally differ with respect to which early-deafened subjects were included. The aim of this second part of our systematic review was therefore to combine the outcomes of the available prognosis studies in early-deafened, late-implanted adolescent and adult $\mathrm{Cl}$ users meeting our criteria, in order to identify the preimplantation factors that affect postimplantation performance. This information could provide guidance for professionals, and be very useful when counseling and selecting new, earlydeafened $\mathrm{Cl}$-candidates.

\section{METHODS}

The same systematic search as performed in Part 1 of the present systematic review, was used to identify the studies eligible for Part 2. We therefore refer to Part 1 for a comprehensive description of the eligibility criteria, search strategy and study selection. In brief, the study population had to consist of $\mathrm{Cl}$ users who received a cochlear implant as adolescents or adults and had a pre- or perilingual age at onset of deafness. The main difference in eligibility criteria compared to Part 1 is related to the outcomes: in order to be included in Part 2, studies had to evaluate the influence of one or more patient characteristics on a measure of speech perception, sound perception, or lipreading. After the results of the systematic search in the five databases had been screened on title and abstract, the full-text versions of the remaining articles were evaluated against the eligibility criteria of Part 2 by 2 independent reviewers; discrepancies between the reviewers were resolved by consensus, after discussion. Next, the relevant data of the resulting, included articles was extracted: the investigated prognostic factors and the corresponding outcome measure were identified, as well as the result of the statistical analysis assessing the strength of the relation between them. Study quality and risk of bias was evaluated by means of the Quality In Prognosis Studies or QUIPS tool (Hayden, Cote, \& Bombardier, 2006; Hayden, van der Windt, Cartwright, Cote, \& Bombardier, 2013). Quality assessment was performed by two independent reviewers and discrepancies were resolved by consensus after discussion. 


\section{RESULTS AND DISCUSSION}

\section{Search and Study Selection}

The systematic search described in Part 1 of this systematic review resulted in 186 articles after the first screening based on study title and abstract. Further assessment of the fulltexts yielded 21 studies that contained (some) prognostic data. Of these 21 studies, 13 were identified as having a clear prognostic study goal, and form the core of this review. The remaining 8 studies (Bosco et al., 2013; Craddock et al., 2016; Kaplan et al., 2003; Kos et al., 2009; Kumar et al., 2016; Peasgood et al., 2003; Shpak et al., 2009; Teoh et al., 2004a) only performed some prognostic analysis as an aside. They were not excluded, but their data will only be used when they are able complement the data of the 13 core studies.

\section{Quality Assessment: Quality in Prognostic Studies (QUIPS) tool}

The QUIPS tool was completed for those 13 studies that were identified to have a clear prognostic study goal. For the 8 studies that only performed some prognostic analysis as an aside we chose not to complete the QUIPS tool. Table 1 presents an overview of the QUIPS outcomes and more details on the judgment per item can be found in Appendix B. The original QUIPS domain regarding risk of bias due to confounding was omitted, given that all 13 studies were of a single-group design. This resulted in 5 bias domains.

Overall, we judged 5 of the 13 studies not to be at high risk of bias in any domain (Caposecco et al., 2012; Klop et al., 2007; Kraaijenga et al., 2016; van der Marel et al., 2015; Yang et al., 2011), 5 to be at high risk of bias in 1 domain (Heywood et al., 2016; O'Gara et al., 2016; van Dijkhuizen et al., 2016; Yucel \& Sennaroglu, 2007; Zeitler et al., 2012), 2 studies to be at high risk in 2 domains (Rousset et al., 2016; van Dijkhuizen et al., 2011) and finally 1 study to be at high risk of bias in 3 domains (Arisi et al., 2010).

\section{Outcomes}

A summary of all 21 included studies, providing general information on the main subject characteristics, predicted outcome measure(s) and reported prognostic outcomes, can be found in Appendix A. As indicated, the focus of this review is on the 13 studies with an explicit prognostic goal. Of those 13, 5 performed a multivariable regression analysis, to identify which independent variables, among a larger set, have a significant relationship to the outcome measure after adjusting for other important variables (Caposecco et al., 2012; Kraaijenga et al., 2016; O'Gara et al., 2016; Rousset et al., 2016; van Dijkhuizen et al., 2011). Seven studies performed correlation analyses, assessing the influence of a single prognostic factor (PF) on the outcome without taking other factors into account, or compared the outcome of groups which were formed based on a certain patient characteristic to see if this had a differential effect on outcomes (Arisi et al., 2010; Heywood et al., 2016; Klop et al., 2007; van der Marel et al., 2015; van Dijkhuizen et al., 2016; Yang et al., 2011; Yucel \& Sennaroglu, 2007). 
Table 1. Overview of the risk of bias based on the Quality in Prognostic Studies tool

\begin{tabular}{|c|c|c|c|c|c|}
\hline Author & $\begin{array}{l}\text { 1. Study } \\
\text { participation }\end{array}$ & $\begin{array}{l}\text { 2. Study } \\
\text { attrition }\end{array}$ & $\begin{array}{l}\text { 3. Prognostic } \\
\text { Factor } \\
\text { Measurement }\end{array}$ & $\begin{array}{l}\text { 4. Outcome } \\
\text { Measurement }\end{array}$ & $\begin{array}{l}\text { 5. Statistical } \\
\text { analysis and } \\
\text { Reporting }\end{array}$ \\
\hline Arisi et al. 2010 & High & Moderate & High & Moderate & High \\
\hline $\begin{array}{l}\text { Caposecco et al. } \\
2012\end{array}$ & Low & $N A^{*}$ & Moderate & Moderate & Low \\
\hline $\begin{array}{l}\text { Heywood et al. } \\
2016\end{array}$ & Moderate & $N A^{*}$ & High & Low & Moderate \\
\hline Klop et al. 2007 & Moderate & Moderate & Low & Low & Moderate \\
\hline $\begin{array}{l}\text { Kraaijenga et al. } \\
2016\end{array}$ & Moderate & $N A^{*}$ & Moderate & Moderate & Moderate \\
\hline $\begin{array}{l}\text { O'Gara et al. } \\
2016\end{array}$ & Moderate & $N A^{*}$ & Moderate & Moderate & High \\
\hline $\begin{array}{l}\text { Rousset et al. } \\
2016\end{array}$ & High & $N A^{\dagger}$ & Moderate & Moderate & High \\
\hline $\begin{array}{l}\text { Van der Marel et } \\
\text { al. } 2015\end{array}$ & Moderate & $N A^{\dagger}$ & Moderate & Moderate & Low \\
\hline $\begin{array}{l}\text { van Dijkhuizen et } \\
\text { al. } 2011\end{array}$ & High & Low & Low & Low & High \\
\hline $\begin{array}{l}\text { van Dijkhuizen et } \\
\text { al. } 2016\end{array}$ & High & Moderate & Low & Low & Low \\
\hline Yang et al. 2011 & Moderate & $N A^{\dagger}$ & Moderate & Moderate & Moderate \\
\hline $\begin{array}{l}\text { Yucel \& } \\
\text { Sennaroglu } 2007\end{array}$ & High & $N A^{\dagger}$ & Moderate & Moderate & Moderate \\
\hline Zeitler et al. 2012 & Low & NA* & High & Moderate & Moderate \\
\hline
\end{tabular}

The remaining study (Zeitler et al., 2012) also performed correlation analyses and group comparisons, each time correcting for the influence of one independent variable. Table 2 presents an overview of these 13 studies including the various predictors and outcome measures used. The strength of the observed relation between predictor and outcome study is expressed by the symbol: from "-", indicating no relation, to "++", indicating a significant relation in a multivariable regression (see the table legend for further details).

Predicted outcomes also differed between studies (see Table 2 and Appendix A), with $\mathrm{Cl}$ word scores being the most common outcome measure; most studies predicted absolute outcome scores, but some predicted the postoperative gain, or it was unclear (Caposecco et al., 2012).

In total, 29 different predictors were used in these 13 studies. We divided them into 4 categories in Table 2, which determine the structure of the detailed overview given below: general predictors, predictors related to the hearing loss, predictors related to hearing aid 
use and performance, and predictors related to the cochlear implantation. More details on the exact correlations and $p$-values can be found in Appendix A.

\section{a. Predictors related to general patient characteristics}

The general patient characteristic gender was not significantly related to the outcomes with $\mathrm{Cl}$ in 3 studies (Table 2). The effect of education was assessed in 2 studies: Klop et al. (2007) found a weak, non-significant correlation with the attained eduaction level, whereas Yang et al. (2011) found that subjects having attended general school performed significantly better compared to subjects having attended schools for the deaf. An important mediating factor for the latter study is of course the level of auditory functioning, with subjects having good auditory performance being more likely to attend general school. Receptive language skills showed a significant, moderate correlation with $\mathrm{Cl}$ outcome in 1 study, but a correction for multiple comparisons was not applied (Rousset et al., 2016). Therefore, for both education and receptive language skills, the amount of available evidence is too scarce to judge whether or not they might be able to predict outcomes.

The degree of preoperative speech intelligibility generally showed to be significantly related to $\mathrm{Cl}$ outcome (Table 2); in only 1 study the correlation lost its significance after correction for multiple comparisons (Klop et al., 2007), likely due to the small sample size. In a subsequent analysis on the predictive value of preoperative speech intelligibility measured by means of a Vowel Identification Test (VOW), van Dijkhuizen et al. (2016) determined that a cut-off score of $60 \%$ could maximally discriminate between subjects obtaining "above-chance" versus "chance level" speech perception performance. The QUIPS tool identified a number of points of concern, though. First, the results of the multivariable regression analyses of both O'Gara et al. (2016) and van Dijkhuizen et al. (2011) (indicated by the "!" in Table 2) need to be interpreted with caution, due to the high number of included PF's compared to the number of subjects. Second, both studies by van Dijkhuizen $(2011,2016)$ are at high risk of selection bias: only patients with sufficiently intelligible speech were selected for implantation, which implicates that their results cannot be generalized to very poor speakers, as is acknowledged by the authors. Third, there is a high risk of PF measurement bias in Heywood et al. (2016), since information on the measurement method for speech intelligibility was lacking. Despite these limitations, we conclude that the amount of evidence still indicates that preoperative speech intelligibility has high potential of being a valuable predictor for $\mathrm{Cl}$ outcomes in this patient group, and can be used to guide patient expectations to a certain degree.

Six studies have examined the influence of communication mode in adulthood on $\mathrm{Cl}$ performance. Four studies failed to find a significant relation (Heywood et al., 2016; Klop et al., 2007; van Dijkhuizen et al., 2011; van Dijkhuizen et al., 2016), whereas 2 studies (Rousset et al., 2016; Yang et al., 2011) found that subjects using oral communication 
performed significantly better than subjects using total or manual communication and 1 study (O'Gara et al., 2016) found communication mode to be a significant predictor in a multivariable regression model. In 2 of these studies the QUIPS tool identified problems with respect to how communication mode was measured: Heywood et al. (2016) again did not give any information on the measurement method, and O'Gara et al. (2016) used a rather questionable measurement method, leading to a high risk of bias: their results therefore need to be interpreted with caution. Communication mode in childhood was significantly and independently related to gain in performance after $\mathrm{Cl}$ in 2 studies with relatively large subject numbers (Caposecco et al., 2012; Zeitler et al., 2012), whereas correlations with this prognostic factor (PF) in both studies by van Dijkhuizen et al. (2011, 2016) were weak and nonsignificant. The latter result might be biased by the highly selected subject group of van Dijkhuizen et al. (2011, 2016), which did not contain any signing subjects. On the other hand, the subject groups of Caposecco et al. (2012) and Rousset et al. (2016) did not contain any signers either, and they did find a significant relation, although a high risk of selection bias is present for the latter study as well (see Appendix B). In summary, communication mode is related to $\mathrm{Cl}$ outcome in the majority of studies that included this $\mathrm{PF}$, the relation being more consistent for childhood than for adulthood.

The underlying hypothesis of why preoperative speech intelligibility might predict $\mathrm{Cl}$ outcome, is that the amount of (adequate) auditory input in the first years of life affects both the functional organization of the central auditory system (Kral \& Sharma, 2012; Kral et al., 2017) and the development of speech production skills, and consequently speech intelligibility. If early auditory input is optimized, for instance through optimal use of residual hearing, this might on the one hand reduce the functional decoupling of cortical auditory areas, thus allowing for both bottom-up and top-down information streams necessary for speech understanding through a $\mathrm{Cl}$ (Kral et al., 2017), and on the other hand promote speech production through auditory feedback. This common driving force, as phrased by van Dijkhuizen et al. (2016), might explain the correlation. The same hypothesis may account for the observed relations with both PFs concerned with communication mode: the choice for an oral communication mode assumes the optimal use of residual hearing, thus maximizing the auditory input. The fact that communication mode in childhood was more consistently related to outcomes than communication mode in adulthood, might simply reflect the fact that communication mode can change in the course of a person's life, with a greater importance of communication during sensitive periods early in life. On the other hand, evidence also points to the importance of a good first language acquisition for later language outcome with a $\mathrm{Cl}$, even if this first language is sign language (Campbell, MacSweeney, \& Woll, 2014). Having an oral communication mode does not say anything on the obtained proficiency of spoken language; if an earlydeaf person communicates orally, but has not obtained an adequate language level, this might equally hamper $\mathrm{Cl}$ outcomes. As mentioned earlier, only 1 study was found that 
assessed language level as a PF; we therefore suggest future studies to assess communication mode along with the obtained proficiency in this communication mode in order to shed more light on the complex relation between both PFs.

\section{b. Predictors related to the hearing loss}

Etiology or cause of deafness was not related to outcomes with $\mathrm{Cl}$ in 2 studies: the PF was not significant in the univariable regression performed by Kraaijenga et al. (2016) and the initial significant effect of etiology on CNC improvement score shown by Zeitler et al. (2012), lost its significance when corrected for length of deafness. We hypothesize that factors other than etiology become more important in case of a long duration of deafness with an early onset.

Six studies incorporated age at onset of deafness/hearing loss as PF: 5 of them found no effect or a low correlation which lost significance after Bonferroni correction. This can likely be explained by the fact that the age at onset does not differ much between subjects in the early-deafened group, reducing its predictive capabilities. In addition it is hard to measure this PF in a valid way for subjects who are now adults. Only 1 study (Arisi et al., 2010) found a relatively high and significant correlation between age at deafness diagnosis and $\mathrm{Cl}$ performance, but this study has some clear differences with other studies that may contribute to this finding. First, all of their subjects were implanted as adolescents and not as adults and second, they used age at "diagnosis" of deafness, which is more directly linked to the start of an intervention (e.g. hearing aids) then age at "onset". Although age at diagnosis should be better traceable retrospectively, the QUIPS tool identified problems with the definition and measurement setting of this PF in this study. Summarized, the age at onset of deafness/severe hearing loss is not a valuable predictor, whereas the age at diagnosis of deafness/severe hearing loss appears to be worth further investigation, especially when subjects have smaller durations of deafness.

Duration of deafness was assessed as PF in 8 of the 13 prognostic studies and in all but one, a significant effect on $\mathrm{Cl}$ performance was lacking. One would expect that in studies in this population, the duration of deafness or severe hearing loss at implantation is more or less equal to the chronological age, plus 4 to 6 years maximally. This is indeed the case in 6 out of the 8 studies that included duration of deafness as PF, and they all failed to find a significant relation (Klop et al., 2007; Kraaijenga et al., 2016; van der Marel et al., 2015; van Dijkhuizen et al., 2011; van Dijkhuizen et al., 2016; Yang et al., 2011). In the 2 remaining studies (Caposecco et al., 2012; Zeitler et al., 2012) a differentiation is made between the age at which severe hearing loss occurred and the age at which this evolved to deafness, sometimes several years later. Consequently, the duration of deafness was much shorter for part of the subjects in these studies. Their results are not in agreement however: Zeitler et al. (2012) found a significant effect of length of deafness (range 0.25$17 \mathrm{yrs}$ ) in a group of 54 subjects implanted in adolescence, which remained significant when corrected for age at implantation (range 10-17 yrs), whereas Caposecco et al. (2012) 
did not find an effect of duration of deafness (range 3-65 yrs) in a group of 34. As a critical note, Zeitler et al. (2012) did not define "deafness", leading to a high risk of bias (see Table 2 ). Due to the applied differentiation between age at severe hearing loss and age at deafness, the latter 2 studies could be considered as more representative for the next PF in Table 2, progressivity of hearing loss. When Caposecco et al. (2012) divide their subjects in 2 groups based on progressivity, this dichotomic variable turns out to be a significant predictor in their regression model, whereas duration of deafness as continuous predictor, was not. This is unexpected, because a lot of the original information is discarded when using a dichotomization, and it is therefore actually less likely to observe a significant relation. On the other hand, O'Gara et al. (2016), who also assessed progressivity by means of a dichotomous variable, did not find a significant effect of the progressivity of the hearing loss. These authors did acknowledge having difficulties assigning patients to one of both groups, since this was largely depending on patient reports, making it more prone to bias. Additionally the study by Bosco et al. (2013), which does not belong to the core group of 13 studies, found that patients with a perilingual onset of deafness performed significantly better than patients with a prelingual onset. Taken together, we can say that the duration of severe hearing loss, closely related to the chronological age at implantation, does not seem to be relevant in predicting $\mathrm{Cl}$ outcomes in early-deafened subjects. The duration of deafness, which is mediated by the potential progressivity of the hearing loss, or another measure of progressivity, might have potential in predicting outcomes, although more research is definitely needed.

The study by Arisi et al. (2010) was the only one to assess the effect of the degree of hearing loss at onset on outcomes, although we could say that it is yet another way to define progressivity of the hearing loss, which is discussed above. A strong, significant correlation with mean speech perception scores after $\mathrm{Cl}$ was observed. However, this result needs to be nuanced given the high risk of measurement bias for this PF (the use of pure-tone audiometry to assess the degree of hearing loss at onset, i.e. in very young children, is highly unlikely).

The next 5 investigated prognostic factors in Table 2 are all related to preoperative hearing thresholds, with varying definitions. In Arisi et al. (2010), a strong negative, significant correlation was found, but it was unclear to which ear the hearing loss belonged. Nonsignificant correlations with the preoperative PTA of the implanted ear were found by both Klop et al. (2007) and Rousset et al. (2016); the latter equally found a nonsignificant correlation with the thresholds of the contralateral ear. In a group of 58, Kraaijenga et al. (2016) found moderate correlations with the preoperative PTA of both the implanted and nonimplanted ear, which were no longer significant when correcting for multiple comparisons. In the same study, the median preoperative PTA of both ears was more significant, although it was not retained in the subsequent backward multivariable analysis either. In summary, results suggest that a relation is likely not 
Systematic review part 2: predictors of performance

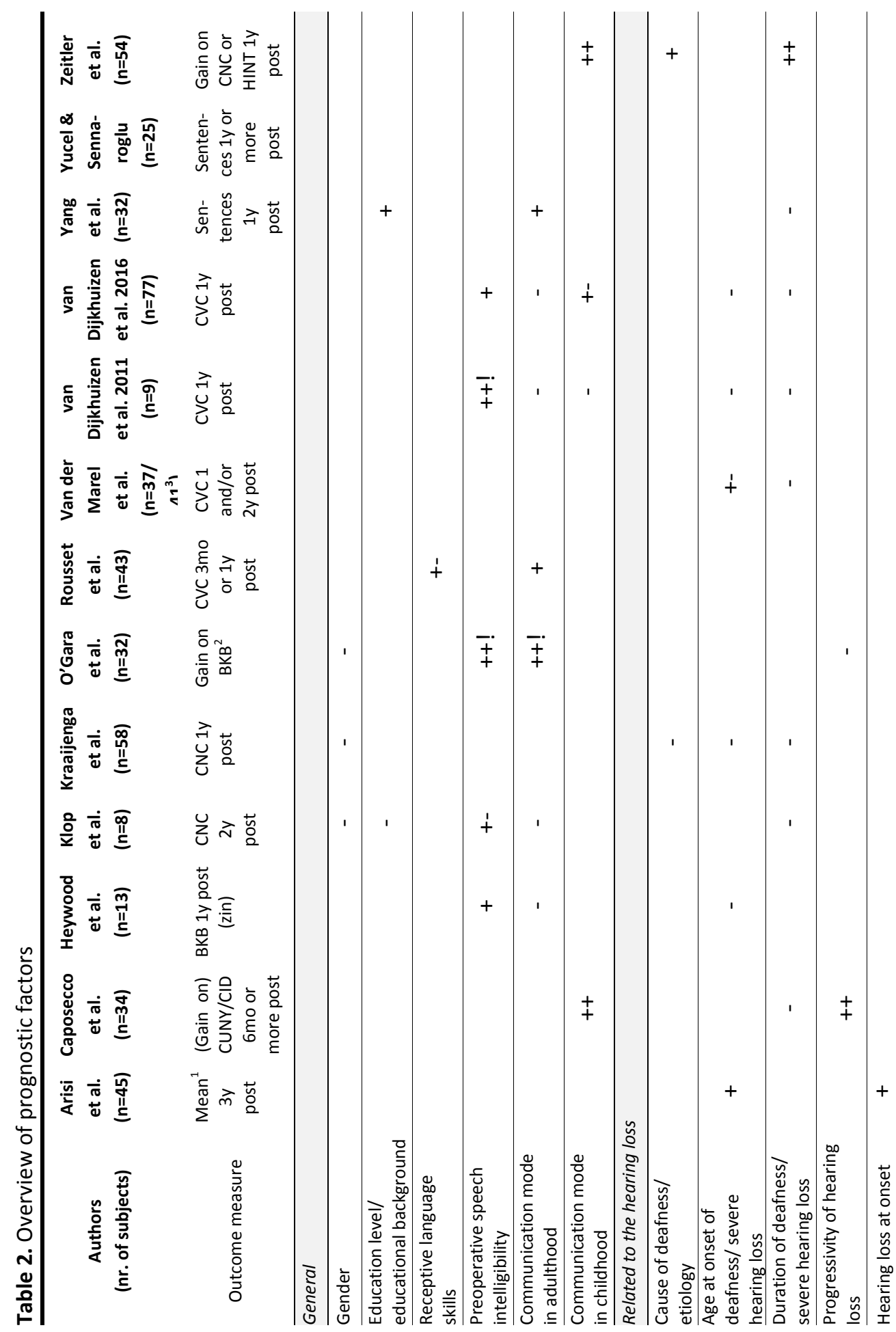




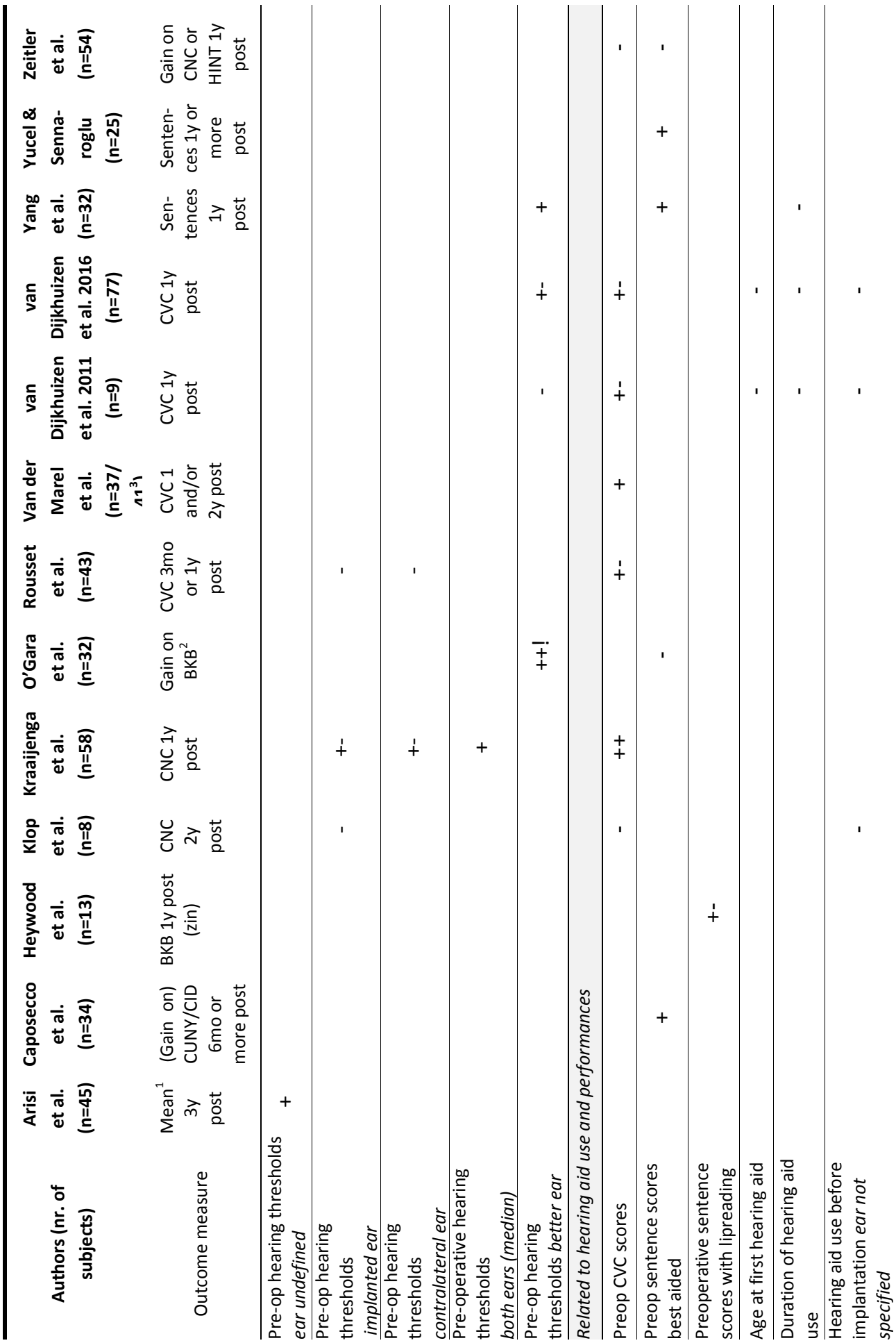




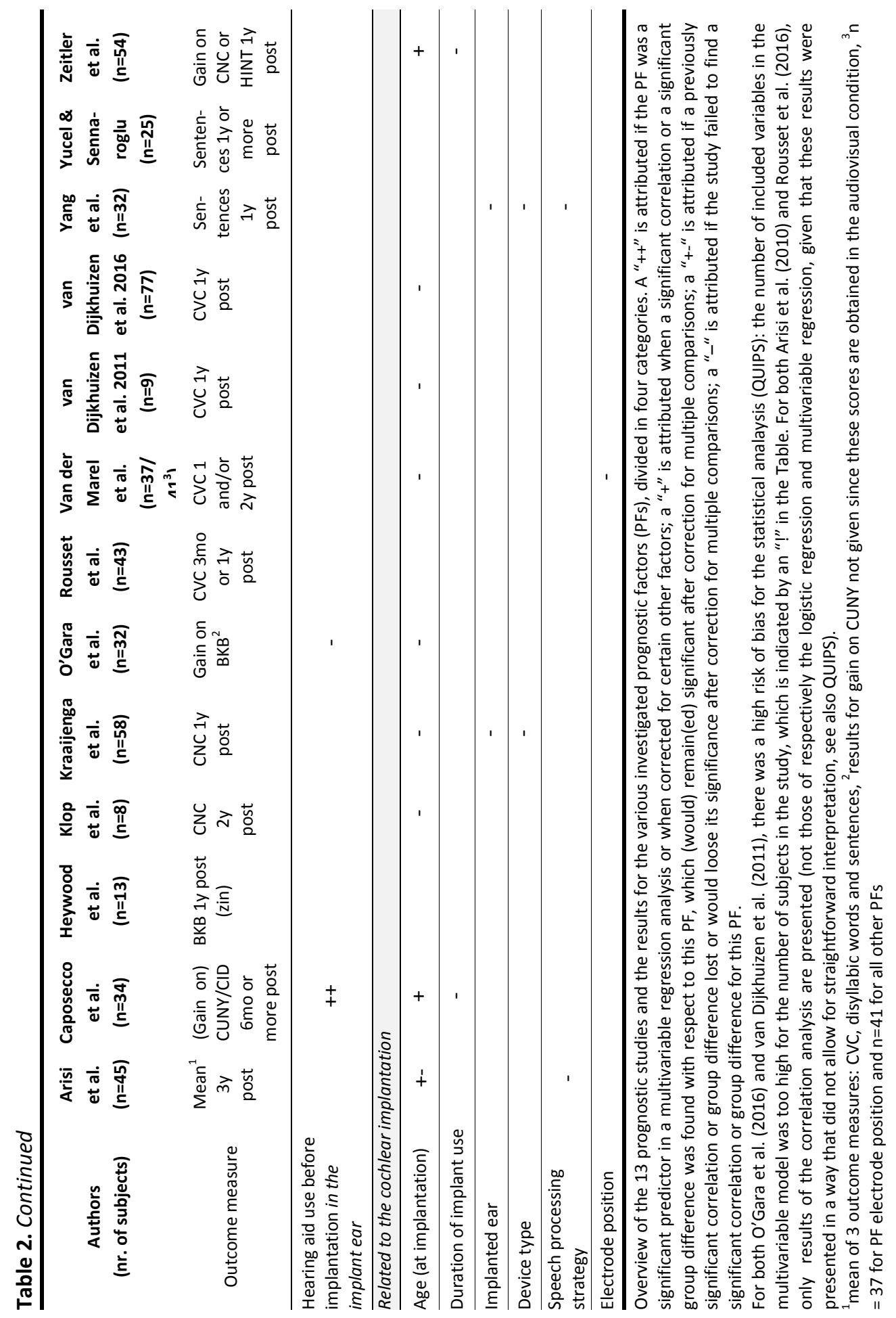


present for the thresholds of the implanted ear; the contralateral ear was included in only 2 studies, not allowing for much interpretation. More extensively investigated is the relation with the preoperative hearing thresholds of the better ear (4 studies): it turned out to be a significant predictor in the multivariable regression model by O'Gara et al. (2016), with high risk of bias however, and had a strong, significant correlation with outcome in the study by Yang et al. (2011), but was on the other hand not related with outcome in both studies by van Dijkhuizen et al. (2011, 2016). The latter might be related to the small variability in thresholds between the subjects in these studies (all needed to have $>90 \mathrm{~dB}$ in order to qualify for $\mathrm{Cl}$ ). For future research it thus seems advisable to focus on preoperative hearing thresholds of the better ear, and to provide a clear definition, in order to complement the available evidence.

\section{c. Predictors related to hearing aid use and performance}

With the exception of Arisi et al. (2010), all prognostic studies included a measure of preoperative speech understanding as PF. Seven studies used CVC or CNC scores; in Kraaijenga et al. (2016), the preoperative CVC score was the only remaining, significant predictor of a backward, multivariable regression analysis, explaining $31 \%$ of the variation in postop CVC scores with $\mathrm{Cl}$, and in van der Marel et al. (2015) a moderate but significant correlation between the pre- and postoperative CVC scores was found, also after Bonferroni correction. In 3 other studies the initially significant correlations did not hold after multiple comparisons correction (Rousset et al., 2016; van Dijkhuizen et al., 2011; van Dijkhuizen et al., 2016): correlations were indeed weak in 2 studies, yet strong in van Dijkhuizen et al. (2011), but the small sample size reduced power. Finally 2 studies failed to find a significant correlation between pre- and postop CVC scores (Klop et al., 2007; Zeitler et al., 2012), the absence of significance for the moderate correlation found by Klop et al. (2007) might also be explained by power issues. In several studies, the condition in which the scores were obtained (best aided, $\mathrm{Cl}$ only, ...) was not mentioned. Six studies investigated whether the preoperative scores on a sentence test could predict the outcome. Both Yang et al. (2011) and Yucel and Sennaroglu (2007) found significant correlations between absolute postop and preop sentence scores. Caposecco et al. (2012) also found a significant correlation between the best preimplant CUNY/CID score and the gain on the CUNY/CID, although this factor failed to retain significance in the subsequent multivariable regression analysis. On the other hand, in the studies by O'Gara et al. (2016) and Zeitler et al. (2012) the gain on respectively the BKB and HINT sentence test was not significantly related to the preop scores. Conversely, Heywood et al. (2016) found a relatively high correlation between the postoperative BKB sentence score and the preoperative CUNY sentence score obtained with lipreading, but a correction for multiple comparisons was not applied.

Taken together, the significant correlations observed by the majority of studies point to a cautious trend that postoperative speech recognition performance can be predicted by 
preoperative performance on that same test. When looking at the 2 studies that failed to find a significant relation, it is striking that they both use the 'gain' in speech perception as outcome measure (O'Gara et al., 2016; Zeitler et al., 2012) instead of the absolute scores. To obtain a high correlation between preop absolute scores and postop gain, you need subjects that have low scores preop to obtain small postop gains, but conversely you need subjects that have high absolute scores preop to obtain large postop gains. The latter could be problematic since ceiling effects might prevent subjects from obtaining large gains. In addition there might be the influence of the test condition, especially whether postop measurements are done with $\mathrm{Cl}$ only or $\mathrm{Cl}+$ hearing aid. In O'Gara et al. (2016), test conditions were best-aided pre- and postoperatively, and the authors acknowledge that there were ceiling and floor effects present in their data which could have skewed the results. In Zeitler et al. (2012), the postop test condition is not given (likely Cl only), nor are the absolute scores given, which makes it impossible to judge whether ceiling effects were present. We note that Caposecco et al. (2012), who did find a significant correlation, probably also used 'gain' as outcome measure, but this is unclear (see also QUIPS). In this study however, the majority of subjects obtained a score near to $0 \%$ preoperatively; consequently there is not much difference between the absolute scores and the gains. Finally, the relatively strong correlation found by Heywood et al. (2016) indicates that even speech understanding scores obtained in an auditory-visual condition might be valuable in predicting postop auditory-only outcomes. The QUIPS tool identified a number of shortcomings with respect to the various speech recognition measures, none of them causing high risk of bias however. Selection bias might be present however for Rousset et al. (2016) and van Dijkhuizen et al. (2011, 2016), as stated previously, and additionally for Yucel and Sennaroglu (2007) (see Table 1 and Appendix B).

The next 4 PFs in this section are related to the hearing aid use itself. Age at first hearing aid was used in the 2 studies by van Dijkhuizen et al. $(2011,2016)$, who both found very weak, nonsignificant correlations. This is somewhat surprising, given that the age at first hearing aid can be regarded as a starting point for auditory rehabilitation and early auditory stimulation is generally regarded as very important for auditory development. A first consideration to be made is that the age at which a child receives hearing aids, does not tell us anything about the quality of the auditory stimulation. Second, the information on this PF was presumably gathered through recall, and might therefore not always be reliable. On the other hand, we previously suggested that the significant correlation between age at diagnosis and $\mathrm{Cl}$ outcome found by Arisi et al. (2010) might be due to the close relation with the start of an intervention, e.g. hearing aids, which is in contrast with the current findings. We therefore suggest that this PF might be worth further investigation, since the limited number of studies including this PF do not allow for a definite conclusion.

The same 2 studies by van Dijkhuizen et al. (2011, 2016) equally observed nonsignificant correlations with duration of hearing aid use, as did a third (Yang et al., 2011). This is not 
unexpected since the number of years an early-deafened person has been using a hearing aid before receiving a $\mathrm{Cl}$ is largely determined by that person's age. This PF therefore predominantly says something about the effect of age at implantation. This problem is not present for the next PF, hearing aid use before implantation, since it was defined as a categorical variable: yes/no by van Dijkhuizen et al. $(2011,2016)$ and the number of hearing aids being worn' (0, 1 or 2) by Klop et al. (2007). None of them observed a significant correlation, however. Finally, 2 studies specifically looked at hearing aid use before implantation 'in the implant ear', also defined as a dichotomous variable: Caposecco et al. (2012) found this PF to be a significant, independent predictor of postoperative results in their multiple regression analysis, whereas O'Gara et al. (2016) did not. Why Caposecco et al. (2012) were the only ones to find a significant relation for a variable related to hearing aid use, which even turned out to be a significant predictor in a multivariable regression analysis, is not clear. O'Gara et al. (2016) hypothesized that the small proportion of subjects not having used a hearing aid in the implant ear in their study, could potentially explain their lack of an effect. A potential problem with the PF hearing aid use before implantation is that it only says something about the moment right before implantation, and nothing about the years before that. We would therefore advise to investigate the effect of "the number of years someone has not been using a hearing aid before implantation", which reduces, though not eliminates, the influence of the patients' age, and does not pinpoint the answer to a single moment in time.

\section{d. Predictors related to the cochlear implantation}

Age (at implantation) is an extensively investigated PF. Van Dijkhuizen et al. used "age at testing" (2011) or "age at first visit to the Cl center" (2016), but since this did not differ much from age at implantation, we judged it legitimate to add both studies to the latter group. In 6 of the then in total 9 studies, no significant relation between age at implantation and $\mathrm{Cl}$ outcome was found (see Table 2). Three studies did observe a relation, which however lost significance when the influence of other variables was taken into account (Caposecco et al., 2012; Zeitler et al., 2012) or would lose significance if correction for multiple comparisons had been applied (Arisi et al., 2010). Looking at these studies in greater detail, it is somewhat striking that the subject groups of both Arisi et al. (2010) and Zeitler et al. (2012) consist solely of adolescents. Caposecco et al. (2012) also have some subjects implanted before the age of 18 , whereas in the remaining studies who did not find a relation, all subjects are older than 18 at the time of implantation. We can therefore say that, for subjects implanted in adulthood, there is very likely no relation between outcomes and age at implantation, whereas for subjects implanted as adolescents, this relation needs to be clarified.

The remainder of PFs related to the cochlear implantation were included in only a limited number of studies each. Neither Caposecco et al. (2012) nor Zeitler et al. (2012) found a significant correlation with the duration of implant use. We must note that in the study by 
Zeitler et al. (2012) this was limited to the length of $\mathrm{Cl}$ use within the first year, whereas Caposecco et al. (2012) failed to show a significant correlation between subjects' outcome at a certain point in time and their duration of implant use at that time, indicating that prolonged use of the $\mathrm{Cl}$ does not further increase performance. The implanted ear was found not to have a significant effect on outcome in 2 studies, although differently defined: Kraaijenga et al. (2016) looked at implantation in the right vs. left ear, whereas Yang et al. (2011) compared performance of implantation in the better vs. poorer ear. A total of 4 studies showed no effect of either speech processing strategy or device type $(\mathrm{Cl}$ brand): Arisi et al. (2010) and Yang et al. (2011) compared the speech processing strategy and found no difference between subjects using CIS vs. SPEAK and CIS vs. ACE, respectively; Kraaijenga et al. (2016) and Yang et al. (2011) compared device type and found no difference in outcome, for either recently implanted patients (between 20062008 in Yang et al. (2011)) or patients implanted from 1988 onwards (Kraaijenga et al., 2016). Finally, electrode position in the cochlea does not seem to have a significant influence on performance (van der Marel et al., 2015): although investigated in just one study, subject numbers were quite high $(n=37)$ and risk of bias for this study, and particularly for this PF, was low. It must be noted, however, that this finding does not apply to subjects with cochlear malformations or incomplete insertions, since they were excluded in this study.

\section{Limitations of the current review}

The main limitations of the review are related to the quality of the included studies, the majority having a high risk of bias on one or more QUIPS items. Due to a lack of information on subject recruitment it is often unclear whether the subject group is representative for our population of interest, which potentially limits the generalizability of the current findings. Similarly, unclear definitions of prognostic factors and outcome measures impeded the analyses, making it more difficult to compare the findings of the different studies. In addition, the quality of the performed and/or reported statistical analyses caused difficulties when interpreting the results, and sometimes had as a consequence that part of the study results had to be omitted. Another problem, although not related to study quality per se, is that outcomes rely on the availability of correct data on the prognostic factors of interest. For example, data on the progressivity of hearing loss of the study subjects will generally rely on recall by family members, and are therefore not very reliable, which again weakens the strength of the evidence.

\section{SUMMARY AND FINAL CONSIDERATIONS}

The current systematic review aimed to identify the factors that are predictive of postoperative outcomes in early-deafened, late-implanted adults or adolescents with a state-of-the-art cochlear implant. Study quality was assessed by means of the QUIPS tool. 
Of the 21 studies that were identified as having assessed the influence of one or more prognostic factors on outcome, 13 had a clear prognostic goal. The results of these 13 studies formed the core of the analysis performed in this review.

Based on the analysis of the 13 core studies, we were able to identify a number of variables that were significantly related to postop outcomes with $\mathrm{Cl}$ in this population and which therefore seem to have good potential in predicting $\mathrm{Cl}$ outcomes. These variables are communication mode (in childhood), preoperative speech intelligibility, and preoperative speech recognition scores. We suggest these variables to be used as prognostic factors in future research in order to develop a model that can more accurately predict $\mathrm{Cl}$ outcomes and can be used for patient counseling and decision making. In addition, we identified or made suggestions for modifications to a number of variables, which have not yet shown to be valuable predictors, but seem promising. We suggest to include these variables in future research in order to clarify whether or not they can fulfill these expectations. These variables are: a measure of the language proficiency in the preferred communication mode, age at diagnosis of severe hearing loss, a measure for duration of deafness which takes progressivity into account, preoperative hearing thresholds of the better ear, the period of time a hearing aid has not been used in the implanted ear, age at implantation (subjects implanted as adolescents only), and age at first hearing aid.

When looking at these promising prognostic factors, it is obvious that the vast majority are somehow related to the impact of early-onset hearing deprivation on the developing auditory structures in the brain; some factors, such as hearing aid use, more residual hearing, or a more progressive hearing loss, might reduce the extent of this impact, while others, such as speech intelligibility, are mediated by the extent of it. The amount of outcome variation that can currently be predicted by the investigated PFs remains limited. We therefore suggest that future research should aim at finding measures of underlying processing skills relevant for auditory functioning, likely affected by auditory deprivation, which can be reliably measured in this population. Such measures could then be used to predict outcomes and potentially explain more of the variation between subjects.

An interesting question is whether there are any similarities between the relevant prognostic factors in early-deafened and postlingually deafened $\mathrm{Cl}$ users. Of the 3 most promising prognostic factors found in this review, however, communication mode and speech intelligibility are specific to the early-deafened population and irrelevant to patients with a postlingual onset of deafness. Conversely, the factor most consistently found to be predictive of outcomes in postlingual patients, the duration of the hearing loss prior to implantation, was extensively investigated but clearly not related to outcomes in the early-deafened population. This can be explained by the similar age at onset for these subjects, which puts duration of hearing loss at the same level as age at implantation. Potentially of importance in both groups is the use of hearing aids preoperatively, although more research on this prognostic factor is required for the early-deafened group. 
Therefore, only one preoperative factor was found to be a relevant predictor throughout the adult population at the moment, i.e. the preoperative amount of speech recognition.

The QUIPS questionnaire identified several weaknesses in the majority of prognostic studies, which could all potentially bias the observed results. It was shown that it is crucial to use a clear and unequivocal definition and measurement method for all prognostic factors, as well as outcome measures, involved in a study. With respect to the outcome measures we suggest to use absolute outcomes (in contrast to gain scores) on a validated test, which is preferably less prone to either ceiling or floor effects.

In conclusion, the results of this review allowed us to identify 3 potential prognostic factors for speech understanding outcomes in early-deafened, but late-implanted adolescent and adult $\mathrm{Cl}$ users. We also made suggestions for a number of additional factors that seem worth further investigation in this patient population. Future studies ideally should have large subject groups, allowing for multivariable regression, and accurately define all investigated variables. 
Chapter 5

APPENDIX A: General Summary of the studies included in Part 2

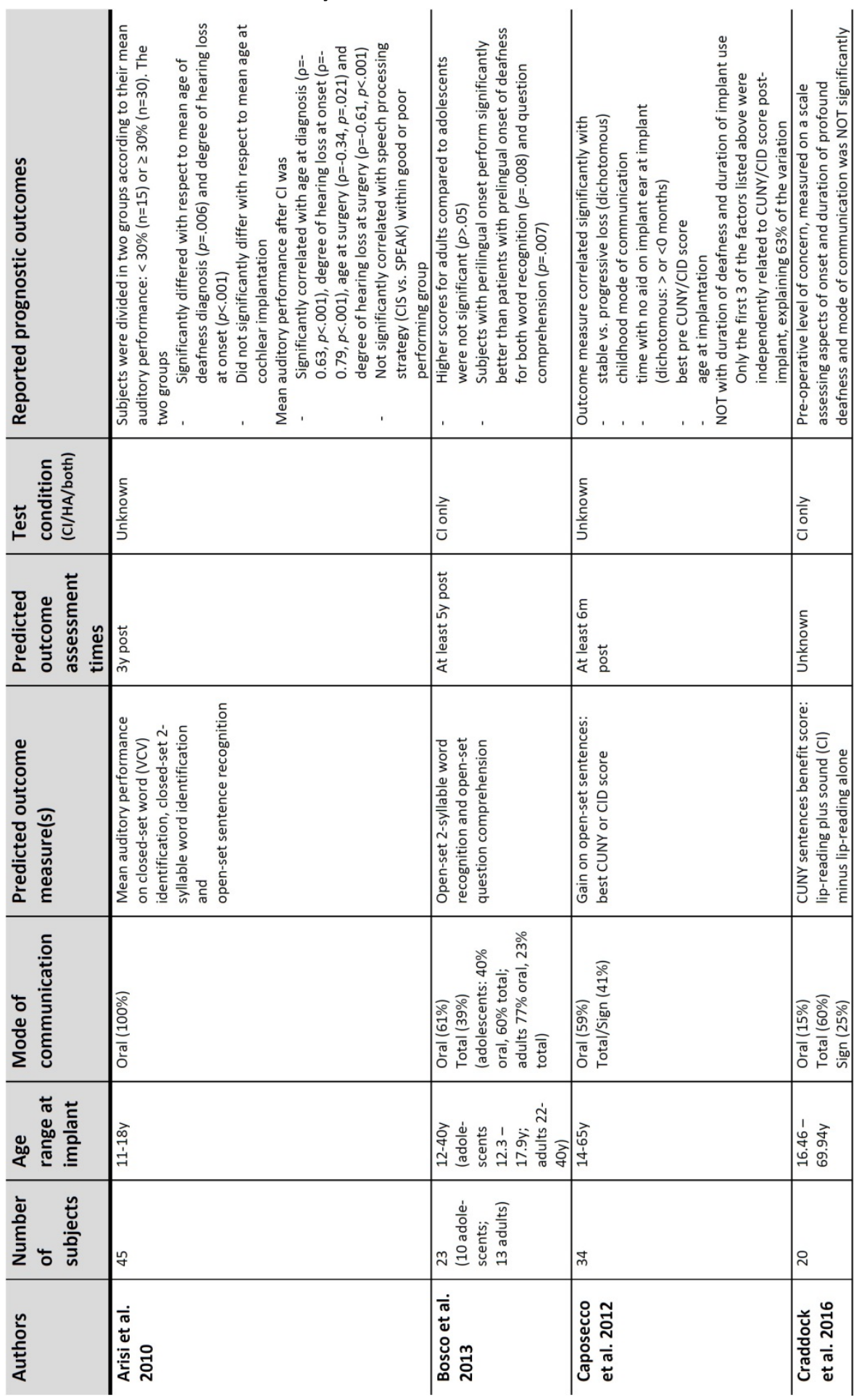


Systematic review part 2: predictors of performance

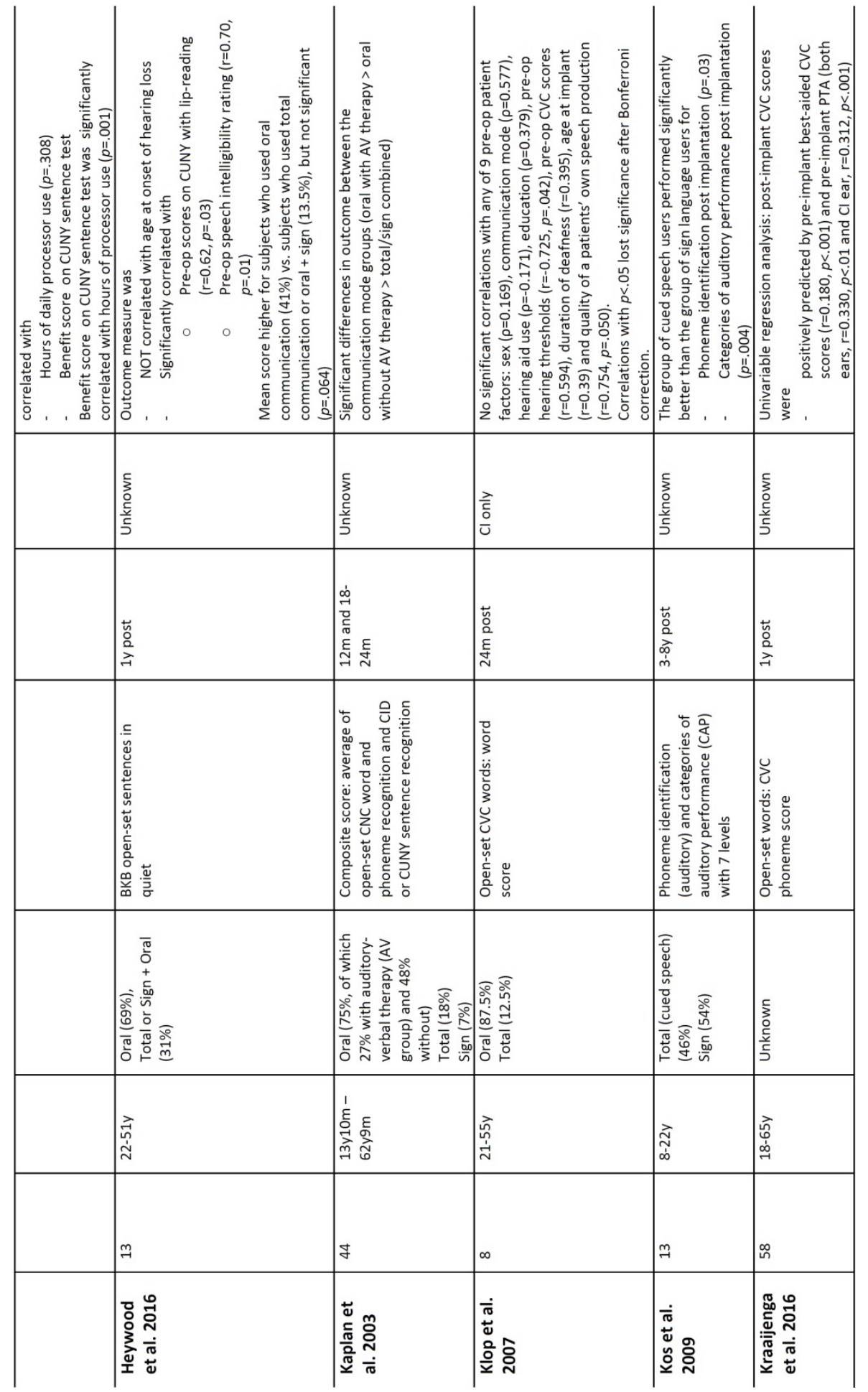


Chapter 5

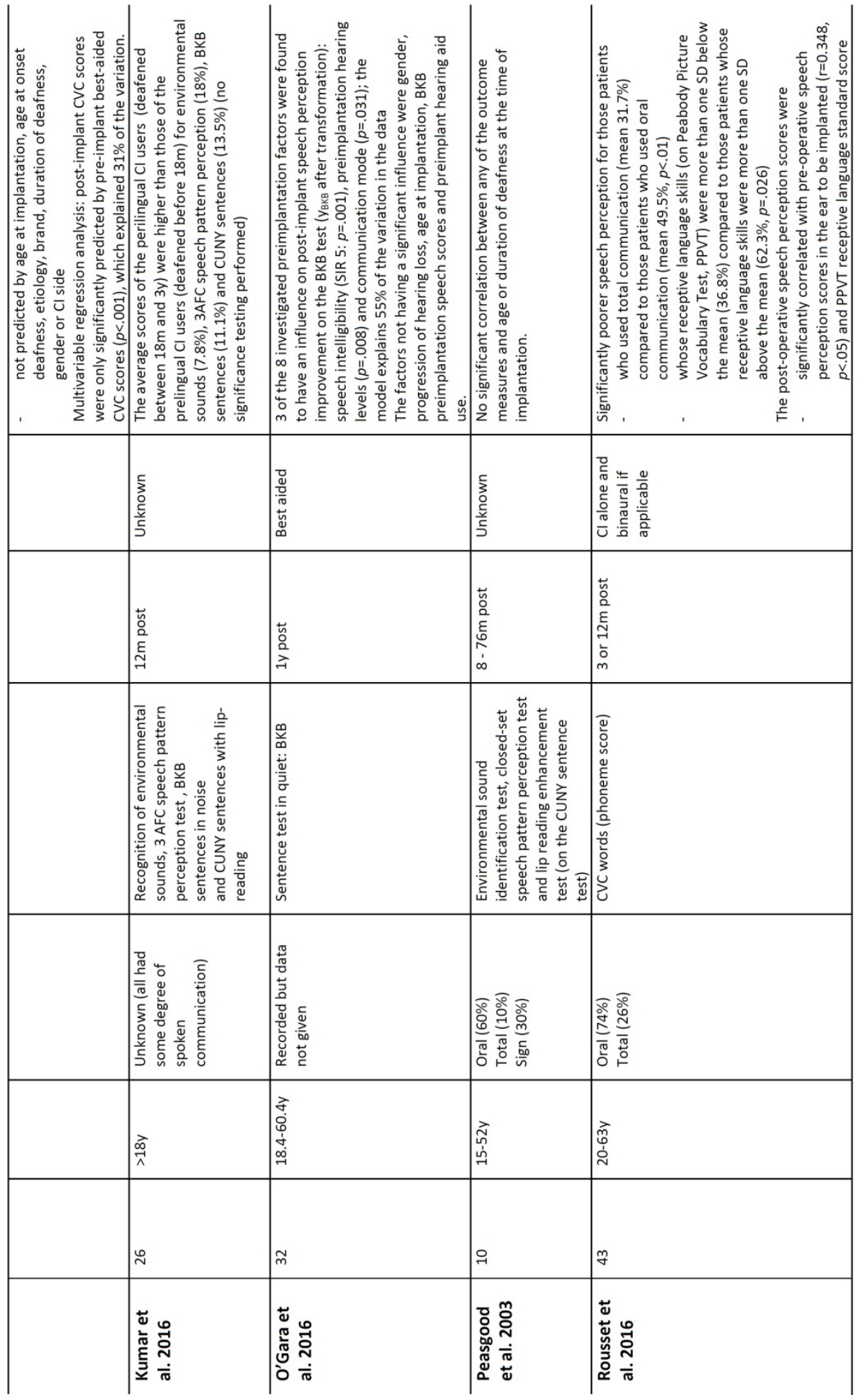




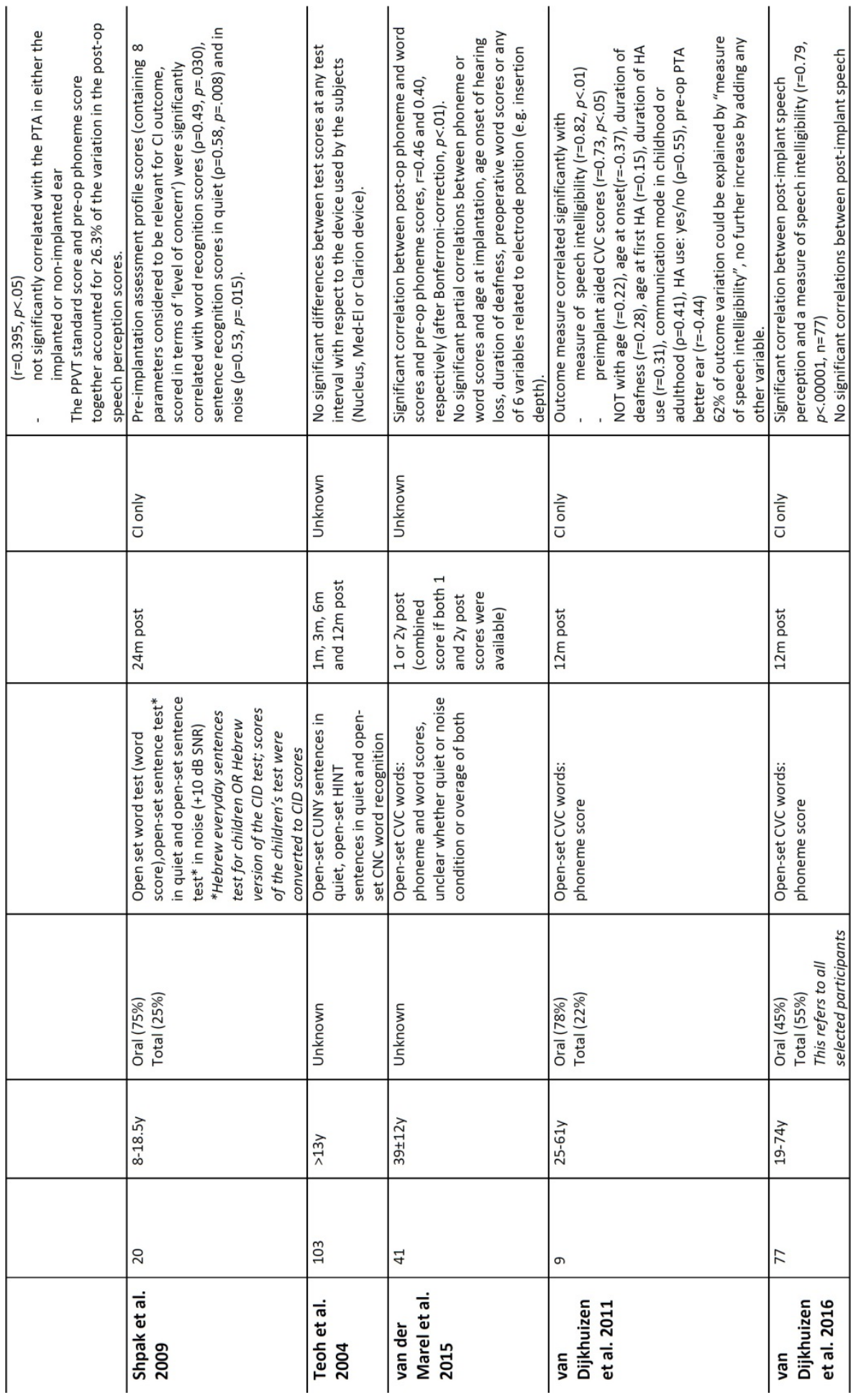


Chapter 5

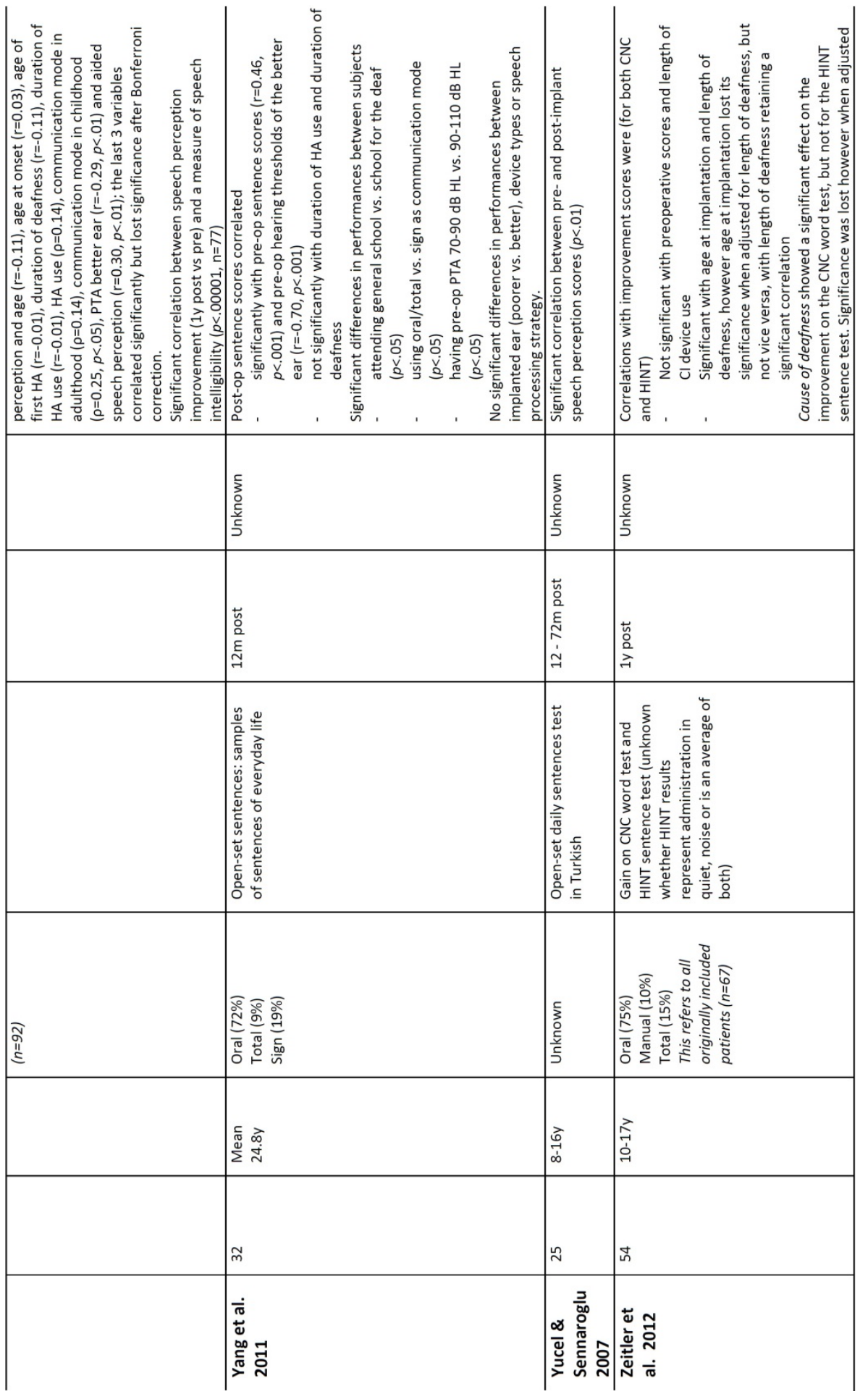


Systematic review part 2: predictors of performance

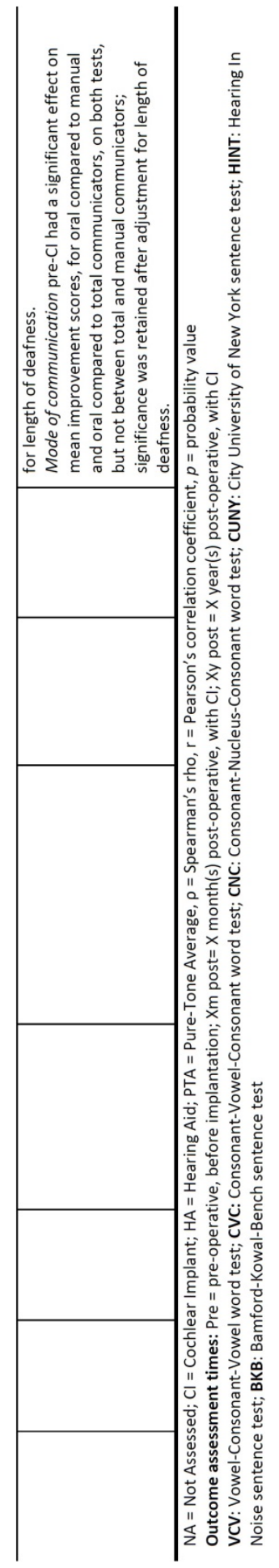


APPENDIX B: Results on the QUIPS tool

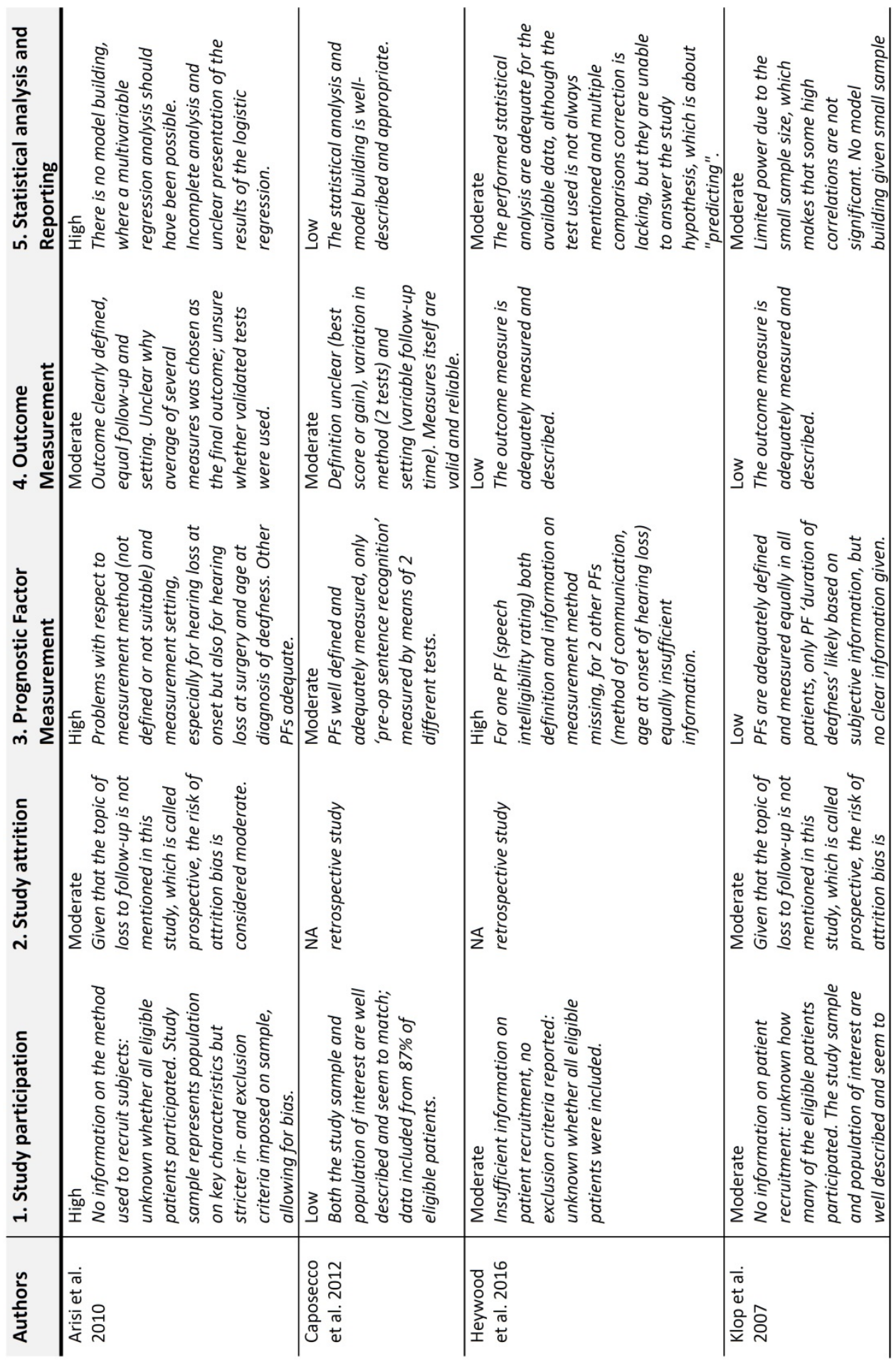




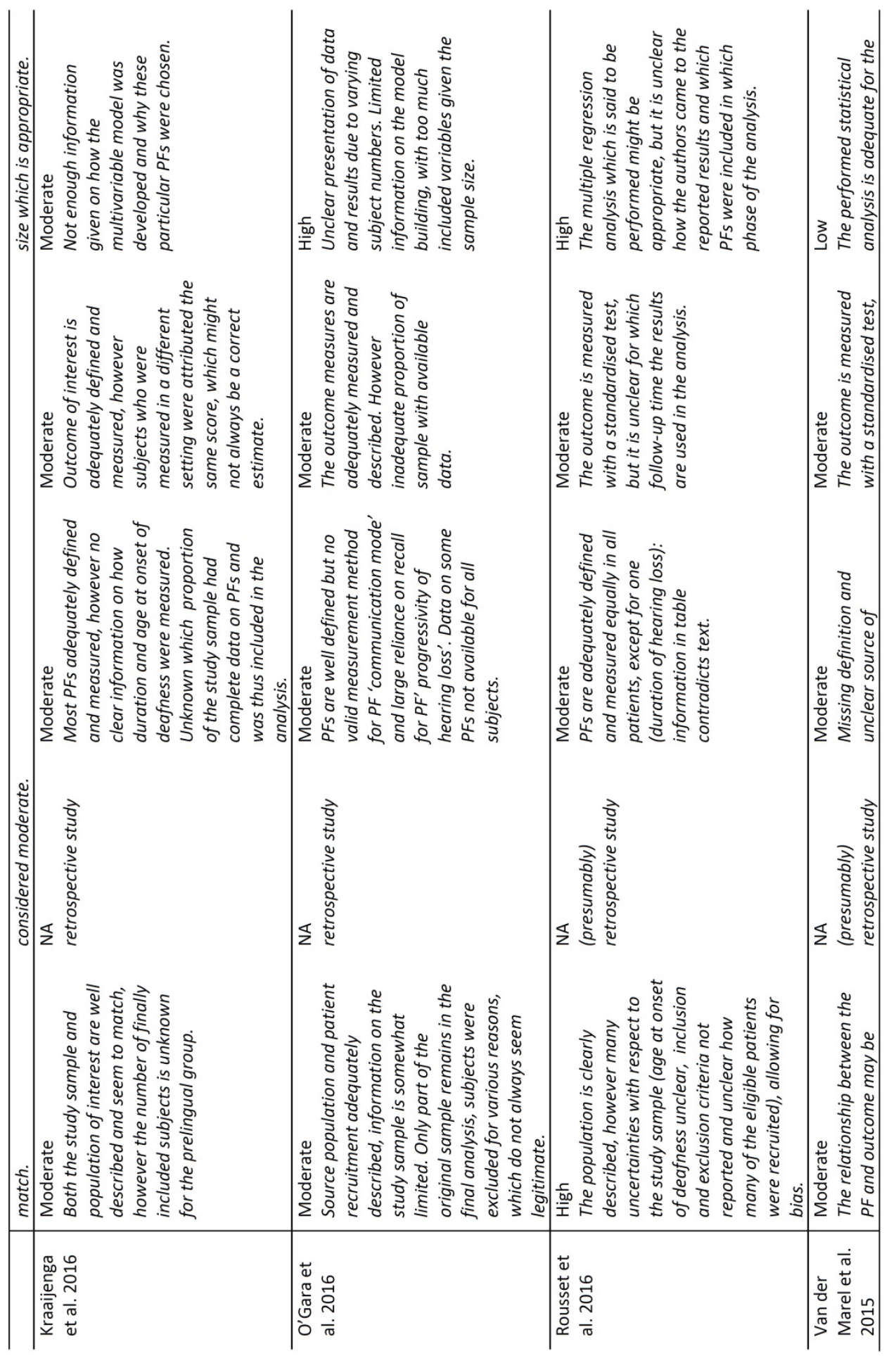




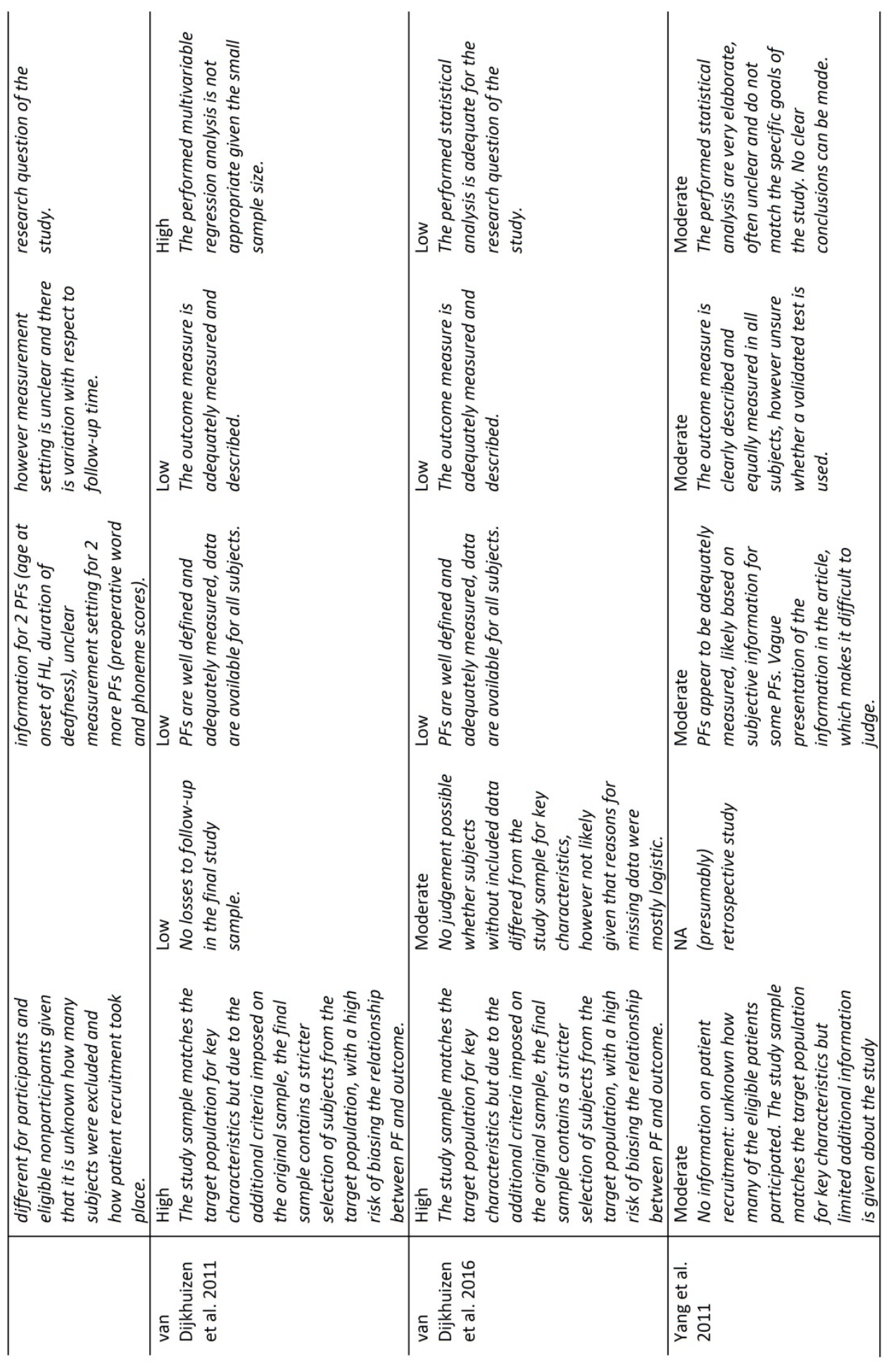




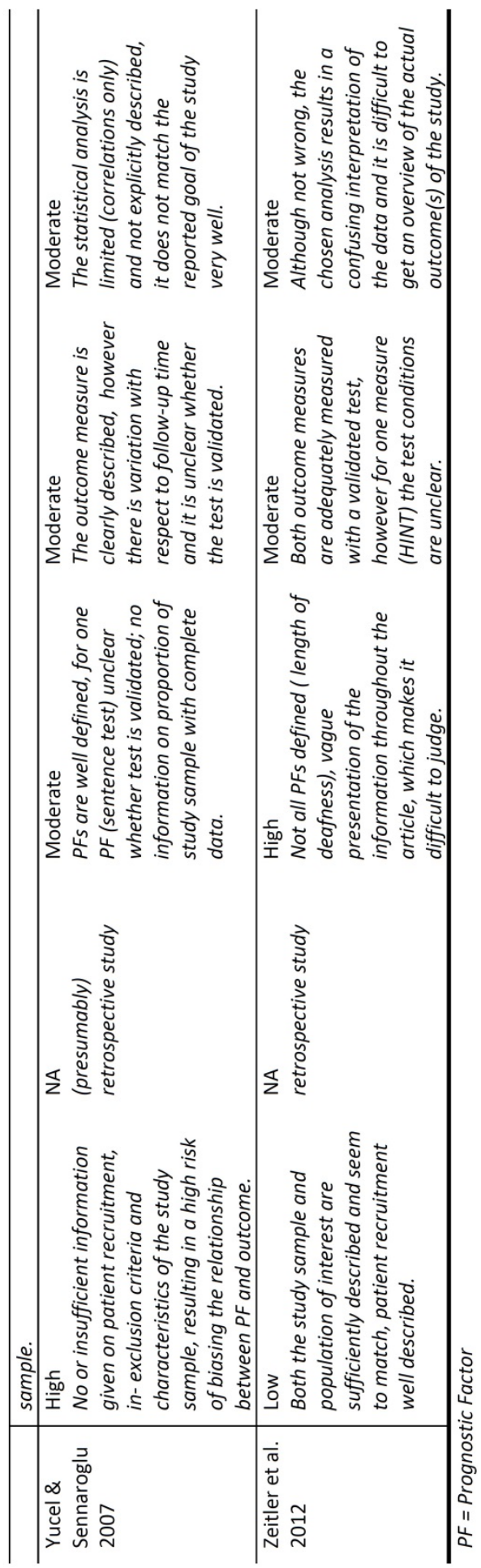



Chapter 6

\section{Late cochlear implantation in early- deafened adults: a detailed analysis of auditory and self-perceived benefits}

Audiology \& Neurotology, 2017, 22: 364-376. DOI: 10.1159/000488023

Joke Debruyne

Miranda Janssen

Jan Brokx 


\section{ABSTRACT}

Objectives: It is known that early-deafened cochlear implant $(\mathrm{Cl})$ users are a very heterogeneously performing group. To gain more insight into this population this study investigated (1) postoperative changes in auditory performance over time based on various outcome measures, focusing on poor performers, (2) self-perceived outcomes, (3) relations between auditory and self-perceived outcomes and (4) preimplantation factors predicting postoperative outcomes.

Methods: Outcomes were assessed prospectively in a group of 27 early-deafened, lateimplanted $\mathrm{Cl}$ users, up to three years post-implantation. Outcome measures included open-set word and sentence recognition, closed-set word recognition, speech tracking and a questionnaire on self-perceived outcomes. Additionally, the relative influence of eight preimplantation factors on $\mathrm{Cl}$ outcome was assessed with linear regression analyses. Results: Significant improvements were found for auditory performance measures and most of the questionnaire domains. Significant changes on the closed-set word test, speech tracking and questionnaire were also found for a subgroup of poor performers. Correlations between auditory and self-perceived outcomes were weak and nonsignificant. Preoperative word recognition and preoperative hearing thresholds, both for the implanted ear, were significant predictors of postoperative outcome in the multivariable regression model, explaining $63.5 \%$ of the variation.

Conclusions: Outcome measurement in this population should be adjusted to the patients' individual performance level and include self-perceived benefit. There is still a need for more knowledge regarding predictors of $\mathrm{Cl}$ outcomes in this group, but the current study suggests the importance of the preoperative performance of the ear to be implanted. 


\section{INTRODUCTION}

In the early years of cochlear implantation, adults with an early onset of deafness, i.e. before (prelingual) or during (perilingual) language development, displayed very limited benefit on speech understanding tests (Skinner et al., 1992; Snik et al., 1997; Waltzman et al., 1992). Consequently, they were often considered poor cochlear implant (Cl) candidates. At the same time, studies reported that, in spite of these poor outcomes, satisfaction rates were high (Hinderink et al., 1995; Kaplan et al., 2003; Peasgood et al., 2003; Zwolan et al., 1996). As speech coding strategies evolved towards SPEAK, CIS and ACE from the late 90's onwards, studies showed that more beneficial results were possible for prelingually deafened adults (Schramm et al., 2002; Waltzman \& Cohen, 1999; Waltzman et al., 2002). A number of recent studies have demonstrated significant postoperative improvement in the mean scores of this group of $\mathrm{Cl}$ users on various speech understanding tests (Bosco et al., 2013; Caposecco et al., 2012; Klop et al., 2007; Rousset et al., 2016; Santarelli et al., 2008; Shpak et al., 2009; van Dijkhuizen et al., 2011; Yang et al., 2011). Nevertheless, there are still large interindividual differences, with part of the early-deafened population obtaining scores slightly above or at $0 \%$ when speech understanding is assessed using open-set words or sentences. In this study we argue that this does not necessarily mean that these $\mathrm{Cl}$ users do not gain any auditory benefit from their $\mathrm{Cl}$, but rather that the tests used to evaluate performance might not be sensitive enough to detect more modest contributions of the implant to speech perception. In 2012, Caposecco et al. (2012) showed that while 18 of $38 \mathrm{Cl}$ users with early-onset hearing loss were unable to attain more than $30 \%$ open-set speech discrimination postoperatively, they were all able to at least discriminate suprasegmental cues with their $\mathrm{Cl}$. Two other studies showed that poor performing, prelingually deafened $\mathrm{Cl}$ users were able to integrate auditory information from the cochlear implant with visual speech information, obtaining higher scores in the audiovisual condition (Craddock et al., 2016; MoodyAntonio et al., 2005). These studies demonstrate the importance of including the ability to use suprasegmental and audiovisual cues when evaluating the benefit of cochlear implantation in this patient group. In this study we wanted to investigate this further, by means of examining the added value of two non-traditional outcome measures to capture small, but relevant auditory benefit. Although recent studies have shown significant advancements in mean speech understanding scores for early-deafened $\mathrm{Cl}$ users, it is still unclear when these subjects reach their maximum, or so-called performance plateau. Postlingual $\mathrm{Cl}$ users generally do not show further improvement after 1 year or even 6 months of $\mathrm{Cl}$ use (Lenarz, Sonmez, Joseph, Buchner, \& Lenarz, 2012). However, some studies assessing early-deafened $\mathrm{Cl}$ users over time found that auditory performance continued to improve beyond 6 months or even 1 year post-implantation (Santarelli et al., 2008; Shpak et al., 2009; Zeitler et al., 2012), whereas other studies observed no further increases beyond the 1 year evaluation moment (van Dijkhuizen et al., 2016; Waltzman et 
al., 2002) or even earlier (Teoh et al., 2004a). This knowledge is important in order to guide patient expectations, and it may also influence how to design post-implant rehabilitation. In addition to the evaluation of two non-traditional outcome measures, the current study therefore compares outcomes at regular intervals, up to three years postimplantation.

There is increasing agreement that the impact of cochlear implantation should not only be evaluated with respect to changes in speech understanding performance, but also with respect to self-perceived changes in quality of life. High user satisfaction rates have consistently been reported in studies on early-deafened $\mathrm{Cl}$ users, even in subjects with almost negligible gain in auditory performance (Bosco et al., 2013; Caposecco et al., 2012; Hinderink et al., 1995; Kaplan et al., 2003; Peasgood et al., 2003; Zwolan et al., 1996). A number of studies have evaluated quality of life before and after implantation and found significant postoperative improvements, mainly in hearing-related quality of life questionnaires (Klop et al., 2007; Most, Shrem, \& Duvdevani, 2010; Schramm et al., 2002; Straatman et al., 2014; van Dijkhuizen et al., 2011), but also in hearing-related domains of general health status questionnaires (Klop et al., 2007; Straatman et al., 2014). Questionnaires like the Nijmegen Cochlear Implant Questionnaire (NCIQ) (Hinderink et al., 2000) are able to detect postoperative improvements for early-deafened $\mathrm{Cl}$ users. Nevertheless it must be kept in mind that such questionnaires were developed to evaluate the benefit for postlingually deafened $\mathrm{Cl}$ users and may therefore be less appropriate for early-deafened $\mathrm{Cl}$ users with respect to, for example, the wording of questions (e.g. complexity) or the situations that are addressed (e.g. telephone use). In this study, the questionnaire applied is designed specifically to evaluate self-perceived benefit in earlydeafened cochlear implant users. Moreover, the possible relation between self-perceived benefit, and auditory benefit will be investigated, since they are both relevant in defining and measuring "successful implantation". It is pertinent to ask whether both outcome types provide the same information since this might affect patient counseling and possibly implant indications. Studies investigating this specific relation are scarce, however. In 2011, van Dijkhuizen et al. only found a significant correlation between speech perception outcomes and the subdomain "advanced sound perception" of the NCIQ, whereas both Peasgood et al. (2003) and Straatman et al. (2014) found no significant correlations between auditory outcome measures and scores on the Glasgow Benefit Inventory. Additionally, Straatman et al. (2014) found no significant correlations between phoneme benefit scores and the generic HUI3 (Feeny et al., 2002), or the postoperative changes on the $\mathrm{NCIQ}$. The authors hypothesized that prelingually deafened adults, in contrast to postlingually deafened adults, might be satisfied with just minimal improvements in hearing abilities.

So far we discuss here the significance of optimally measuring the outcome of cochlear implantation in this specific patient group. Another objective of this study is directed toward predicting preoperatively which patients will become good and/or satisfied 
cochlear implant users. Relevant patient characteristics can vary in many ways: the communication mode in which the patient has been raised, whether the hearing loss had a pre- or perilingual onset, the amount of residual hearing, whether or not hearing aids have been used, etc. It would be very useful to know which of these preimplantation factors are related to postoperative outcomes. The results of studies performing correlation analysis or multiple regression analysis in groups of prelingual $\mathrm{Cl}$ users have been ambiguous. Study samples are often small and a wide range of patient factors are considered that are not uniformly defined across studies. Although not conclusive over all studies, significant relations have been found between $\mathrm{Cl}$ outcome and patient's own speech intelligibility (van Dijkhuizen et al., 2016), communication mode (Caposecco et al., 2012; Rousset et al., 2016; Yang et al., 2011; Zeitler et al., 2012) and preoperative speech understanding scores (Caposecco et al., 2012; Kraaijenga et al., 2016; Rousset et al., 2016; van der Marel et al., 2015; Yang et al., 2011). At the same time, duration of deafness (Caposecco et al., 2012; Kraaijenga et al., 2016; van der Marel et al., 2015; van Dijkhuizen et al., 2016; Yang et al., 2011) and etiology (Kraaijenga et al., 2016; Zeitler et al., 2012) do not seem to be related to $\mathrm{Cl}$ outcomes in this patient group.

In summary, the current study had four objectives. The first was to examine in detail postoperative changes in auditory performance in a group of late-implanted, earlydeafened adult $\mathrm{Cl}$ users. For this purpose, both standard open-set word and sentence recognition tests were used pre- and postoperatively, as well as two less commonly used tests, hereafter referred to as "non-traditional" tests. The first of these two tests is speech tracking (De Filippo \& Scott, 1978), which evaluates changes in the best-aided, audiovisual communicative abilities. The second is the monosyllable-trochee-spondee test (Erber \& Alencewicz, 1976), which assesses changes in closed-set, (suprasegmental) word recognition. It was hypothesized that these non-traditional tests could demonstrate clear postoperative changes in subjects who show no or only limited improvements on the standard open-set auditory tests. We also wanted to determine if and when auditory performance reaches a plateau in this patient population. Therefore measurements were done up to 3 years post-implantation. A second objective was to implement and evaluate a questionnaire that would be sensitive to the specific, self-perceived advancements of early-deafened $\mathrm{Cl}$ users. The third study objective was to evaluate the relation between changes in auditory performance and changes in the subjective evaluation questionnaire. The final and fourth aim was to identify which preimplantation factors are related to postoperative speech understanding scores with the $\mathrm{Cl}$. The investigated preimplantation factors were: preoperative pure-tone-average hearing loss (PTA) in the better ear, preoperative PTA in the implanted ear, preoperative best-aided word recognition score, preoperative word recognition score in the implanted ear, communication mode at implantation, hearing aid use at implantation, preoperative hearing aid use in the implanted ear and age at onset of deafness (pre- vs. perilingual). 


\section{MATERIALS AND METHODS}

\section{Participants}

This article presents prospective data, gathered since 2010, on 27 adult early-deafened cochlear implant users who received an implant at the Maastricht UMC+. The Medical Ethical Committee of the MUMC+ deemed that the study fulfilled ethical requirements since it was an extension of regular patient care. All subjects who met the following criteria were included in the study: unilateral implantation, acquisition of deafness or severe hearing impairment at or before the age of four years and implantation in adulthood ( $>18$ years). In order to receive a $\mathrm{Cl}$ at the Maastricht UMC+, early-deafened subjects additionally needed to be sufficiently motivated and receptive to auditory communication. There were no further exclusion criteria. Only subjects who had at least 1 year experience with the $\mathrm{Cl}$ were included.

Twenty-seven subjects met the inclusion criteria. The mean age at implantation of was 45 years (range: 20-71 years). A prelingual onset of deafness or severe hearing loss, defined as an age at onset of $\leq 12$ months, was found in 22 or $81 \%$ of subjects; the remaining five (19\%) had a perilingual onset ( $>12$ but $<48$ months). Nineteen subjects $(70 \%)$ were oral communicators, the remaining eight $(30 \%)$ used a combination of oral and manual communication. All but four subjects (85\%) were using at least one hearing aid at the time of the implantation and 18 subjects (67\%) were wearing a hearing aid in the ear that was subsequently implanted. An overview of relevant subject characteristics and a number of summary statistics can be found in Table 1 . All subjects had followed the extensive rehabilitation program which is the standard post-implant care at the Maastricht UMC+, and all but one were regular users of their device. The latter subject, S15, became a nonuser after 1 year of $\mathrm{Cl}$ use because she could not become accustomed to the sound of the implant. The post-implant follow-up time was 3 years for 14 subjects, 2 years for four subjects and 1 year for the remaining nine subjects (mean 2.2 years). Complications occurred neither during $\mathrm{Cl}$ surgery nor during the postoperative rehabilitation period.

\section{Outcome measures}

The preoperative pure-tone-average hearing loss (PTA) at 0.5, 1 and $2 \mathrm{kHz}$ was calculated for each subject, both for the implanted ear and for the best ear (see Table 1). Open-set word recognition was evaluated in free field at 65 and $75 \mathrm{~dB}$ SPL by means of the phoneme score on the Dutch, monosyllabic, consonant-nucleus-consonant (CNC) word test (Bosman \& Smoorenburg, 1995). The maximum percentage correct was gathered in the best-aided auditory-only condition (with one or two hearing aids preoperatively; postoperatively in the condition used in daily life: either $\mathrm{Cl}$ alone or $\mathrm{Cl}+$ contralateral hearing aid) and additionally in the $\mathrm{Cl}$-alone condition. 
Table 1. Overview of subject characteristics

\begin{tabular}{|c|c|c|c|c|c|c|c|}
\hline $\begin{array}{l}\text { Subject } \\
\text { number }\end{array}$ & Sex & $\begin{array}{l}\text { Age } \\
\text { at } \mathrm{Cl} \\
\text { (yrs) }\end{array}$ & $\begin{array}{c}\text { Cause of } \\
\mathrm{HI}\end{array}$ & $\begin{array}{l}\text { Age at } \\
\text { onset of } \\
\mathrm{HI}(\mathrm{mo})\end{array}$ & $\begin{array}{l}\text { Communi- } \\
\text { cation mode } \\
\text { at } \mathrm{Cl}\end{array}$ & $\begin{array}{c}\mathrm{HA} \\
\text { use } \\
\text { at } \mathrm{Cl}\end{array}$ & $\begin{array}{c}\text { HA use } \\
\text { at Cl } \\
\text { (implanted } \\
\text { ear) }\end{array}$ \\
\hline $1^{*}$ & $\mathrm{M}$ & 30 & Unknown & 0 & Oral + manual & yes & yes \\
\hline 2 & $\mathrm{~F}$ & 72 & Unknown & 0 & Oral & yes & yes \\
\hline $3^{*}$ & $M$ & 27 & Unknown & 0 & Oral & yes & yes \\
\hline $4^{*}$ & $\mathrm{~F}$ & 21 & Meningitis & 4 & Oral + manual & no & no \\
\hline 5 & $\mathrm{~F}$ & 71 & Unknown & 0 & Oral + manual & yes & yes \\
\hline 6 & $\mathrm{~F}$ & 63 & Measles & 36 & Oral & yes & yes \\
\hline 7 & $M$ & 33 & Meningitis & 5 & Oral & yes & yes \\
\hline 8 & M & 25 & Unknown & 0 & Oral & yes & yes \\
\hline 9 & $M$ & 48 & Rubella & 0 & Oral & yes & yes \\
\hline $10^{*}$ & $\mathrm{~F}$ & 51 & Unknown & 0 & Oral & yes & yes \\
\hline 11 & $M$ & 61 & Meningitis & 24 & Oral & yes & yes \\
\hline 12 & $\mathrm{~F}$ & 51 & Unknown & 0 & Oral & yes & yes \\
\hline 13 & M & 49 & $\begin{array}{l}\text { Perinatal } \\
\text { asphyxia }\end{array}$ & 0 & Oral & yes & yes \\
\hline $14^{*}$ & $\mathrm{~F}$ & 35 & Meningitis & 29 & Oral + manual & no & no \\
\hline $15^{*}$ & $\mathrm{~F}$ & 26 & Meningitis & 12 & Oral + manual & no & no \\
\hline $16^{*}$ & $\mathrm{~F}$ & 50 & Rubella & 0 & Oral & yes & no \\
\hline 17 & $\mathrm{~F}$ & 46 & Rubella & 0 & Oral & yes & no \\
\hline 18 & $\mathrm{~F}$ & 61 & $\begin{array}{l}\text { Head } \\
\text { trauma }\end{array}$ & 48 & Oral & yes & yes \\
\hline 19 & $\mathrm{~F}$ & 65 & Unknown & 0 & Oral + manual & yes & no \\
\hline 20 & $\mathrm{~F}$ & 57 & Rubella & 0 & Oral & yes & yes \\
\hline 21 & $\mathrm{~F}$ & 33 & $\begin{array}{l}\text { Pendred } \\
\text { syndrome }\end{array}$ & 0 & Oral & yes & yes \\
\hline 22 & $M$ & 40 & $\begin{array}{l}\text { Perinatal } \\
\text { asphyxia }\end{array}$ & 0 & Oral & yes & yes \\
\hline $23 *$ & M & 62 & Unknown & 0 & Oral & yes & no \\
\hline $24^{*}$ & $\mathrm{~F}$ & 31 & $\begin{array}{c}\text { Ear } \\
\text { infections }\end{array}$ & 24 & Oral + manual & no & no \\
\hline 25 & $\mathrm{~F}$ & 55 & Unknown & 0 & Oral & yes & yes \\
\hline 26 & $\mathrm{~F}$ & 20 & $\begin{array}{l}\text { Pendred } \\
\text { syndrome }\end{array}$ & 0 & Oral + manual & yes & yes \\
\hline $27^{*}$ & $M$ & 44 & Unknown & 0 & Oral & yes & no \\
\hline $\begin{array}{l}\text { Summary } \\
\text { / mean }\end{array}$ & $\begin{array}{c}37 \% \\
M \\
63 \% \\
F\end{array}$ & 45 & NA & $\begin{array}{c}81 \% \\
\text { prelingual } \\
19 \% \\
\text { perilingual }\end{array}$ & $\begin{array}{c}70 \% \text { Oral } \\
30 \% \text { Oral + } \\
\text { manual }\end{array}$ & $\begin{array}{c}85 \% \\
\text { yes } \\
15 \% \\
\text { no }\end{array}$ & $\begin{array}{l}67 \% \text { yes } \\
33 \% \text { no }\end{array}$ \\
\hline
\end{tabular}

Subjects with an asterisk $\left({ }^{*}\right)$ belong to the poor performing group. $(\mathrm{Cl}=$ Cochlear Implantation, yrs $=$ years, $\mathrm{HI}=$ Hearing Impairment, $\mathrm{mo}=$ months, $\mathrm{HA}=$ Hearing Aid, PTA = Pure Tone Average at 0,$5 ; 1 ; 2 \mathrm{kHz}$ ) 
Chapter 6

Table 1. Continued

\begin{tabular}{|c|c|c|c|c|c|}
\hline $\begin{array}{l}\text { Subject } \\
\text { number }\end{array}$ & $\begin{array}{c}\text { Pre-op } \\
\text { PTA } \\
\text { (implanted } \\
\text { ear, dB HL) }\end{array}$ & $\begin{array}{l}\text { Pre-op } \\
\text { PTA } \\
\text { (best ear, } \\
\text { dB HL) }\end{array}$ & $\begin{array}{l}\text { Speech } \\
\text { coding } \\
\text { strategy }\end{array}$ & Implant type & $\begin{array}{l}\text { Follow-up time } \\
\text { post } \mathrm{Cl} \text { (yrs) }\end{array}$ \\
\hline $1^{*}$ & 105 & 95 & ACE & Nucleus $\mathrm{Cl} 422$ (SRA) & 2 \\
\hline 2 & 97 & 92 & ACE & Nucleus $\mathrm{Cl}$ 422(SRA) & 2 \\
\hline $3 *$ & 97 & 97 & ACE & Nucleus $\mathrm{Cl}$ 422(SRA) & 3 \\
\hline $4^{*}$ & 118 & 118 & ACE & Nucleus $\mathrm{Cl}$ 24RE(ST) & 3 \\
\hline 5 & 103 & 102 & FS4 & CONCERTO & 3 \\
\hline 6 & 100 & 83 & FSP/FS4 & CONCERTO & 1 \\
\hline 7 & 105 & 105 & ACE & Nucleus $\mathrm{Cl} 24 \mathrm{RE}(\mathrm{CA})$ & 3 \\
\hline 8 & 97 & 97 & FS4 & CONCERTO & 3 \\
\hline 9 & 115 & 107 & ACE & Nucleus $\mathrm{Cl}$ 422(SRA) & 2 \\
\hline $10^{*}$ & 105 & 105 & ACE & Nucleus $\mathrm{Cl}$ 422(SRA) & 3 \\
\hline 11 & 95 & 95 & HiRes-P & $\begin{array}{l}\text { HiRes 90k Adv } \\
\text { HiFocus ms }\end{array}$ & 1 \\
\hline 12 & 115 & 115 & ACE & Nucleus $\mathrm{Cl}$ 24RE(CA) & 3 \\
\hline 13 & 97 & 92 & $\begin{array}{c}\text { HiRes } \\
\text { Optima-S }\end{array}$ & $\begin{array}{l}\text { HiRes 90k Adv } \\
\text { HiFocus ms }\end{array}$ & 1 \\
\hline $14^{*}$ & 117 & 117 & ACE & Nucleus $\mathrm{Cl} 11+11+2 \mathrm{M}$ & 3 \\
\hline $15^{*}$ & 120 & 102 & ACE & Nucleus $\mathrm{Cl}$ 24RE(CA) & 1 \\
\hline $16^{*}$ & 118 & 112 & ACE & Nucleus $\mathrm{Cl}$ 422(SRA) & 1 \\
\hline 17 & 98 & 88 & FS4 & CONCERTO & 3 \\
\hline 18 & 85 & 67 & $\begin{array}{c}\text { HiRes } \\
\text { Optima-S }\end{array}$ & $\begin{array}{l}\text { HiRes 90k Adv } \\
\text { HiFocus ms }\end{array}$ & 1 \\
\hline 19 & 110 & 97 & ACE & Nucleus $\mathrm{Cl}$ 422(SRA) & 3 \\
\hline 20 & 112 & 105 & ACE & Nucleus $\mathrm{Cl}$ 422(SRA) & 1 \\
\hline 21 & 93 & 93 & ACE & Nucleus $\mathrm{Cl}$ 422(SRA) & 3 \\
\hline 22 & 110 & 110 & ACE & Nucleus $\mathrm{Cl}$ 422(SRA) & 3 \\
\hline $23^{*}$ & 115 & 113 & ACE & Nucleus $\mathrm{Cl} 422$ (SRA) & 1 \\
\hline $24 *$ & 112 & 108 & HiRes-S & HiRes 90k HiFocus $1 \mathrm{~J}$ & 1 \\
\hline 25 & 97 & 83 & $\begin{array}{l}\text { HiRes-S } \\
\text { Fid } 120\end{array}$ & HiRes 90k HiFocus $1 \mathrm{~J}$ & 3 \\
\hline 26 & 93 & 93 & ACE & Nucleus $\mathrm{Cl}$ 422(SRA) & 2 \\
\hline $27^{*}$ & 118 & 117 & ACE & Nucleus $\mathrm{Cl}$ 422(SRA) & 3 \\
\hline $\begin{array}{l}\text { Summary } \\
\text { / mean }\end{array}$ & 105 & 100 & NA & NA & 2,2 \\
\hline
\end{tabular}


The second open-set test was a Dutch sentence recognition test, with a variable volume setting according to the subjects' preference, administered in the best-aided, auditoryonly condition. The result is expressed as a syllable score. The third test, speech tracking (ST), is a structured, "conversation-like" task that combines sentence identification with auditory comprehension (De Filippo \& Scott, 1978). The amount of time needed to repeat a text which is read live and out loud to the subject, determines the score, expressed as the number of words per minute (\#wpm). The speech tracking test was administered in the best-aided, auditory-visual condition. The texts used were specifically designed for early-deafened, Dutch $\mathrm{Cl}$ users (Boons \& Debruyne, 2011). In poor performers, the monosyllable-trochee-spondee (MTS) test (Erber \& Alencewicz, 1976) was used as a test for closed-set (suprasegmental) word recognition. This 12 -item test assesses both word identification (word score) as well as identification of suprasegmental cues (suprasegmental score). Administration of the MTS-test was at $65 \mathrm{~dB}$ SPL in the bestaided, auditory-only condition. If no hearing aids were worn in the preoperative condition of the open-set word, sentence or MTS-test, or if the test was not administrable, a score of $0 \%$ was assigned.

A questionnaire to assess subjective outcomes was constructed specifically for earlydeafened $\mathrm{Cl}$ users. The questionnaire consists of five subscales: primary sound processing, sense of safety, ease of communication, social aspects of hearing and self-confidence. Appendix $A$ contains information regarding the development and subsequent reliability analyses. The translation of the questionnaire is presented in Appendix B.

Auditory tests were administered preoperatively, 6 months, and 1 year postoperatively. For some subjects they were also administered at 2 and 3 years postoperatively, according to available follow-up time. Subjects also completed the questionnaire dedicated to earlydeafened $\mathrm{Cl}$ users at each of these evaluation moments, except at 6 months postoperatively.

\section{Data analysis}

To evaluate the postoperative changes in auditory performance up to 3 years postimplantation (first study objective), with decreasing subject numbers over time, multilevel linear regression modeling was performed. The applied covariance structure chosen was based on likelihood ratio testing and the Bayesian information criterion (BIC). Auditory performance changes over a specific time period were then evaluated with pairwise comparisons. Bonferroni-Holm adjustments of the standard $\alpha$-value of 0.05 were made in case of multiple comparisons. If the sample size was small, which was the case when considering a subgroup of subjects, the non-parametric Wilcoxon signed-rank test was applied to pairwise comparisons. Descriptive statistics of the difference or change scores between preoperative and 1 year postoperative measurements were also obtained.

For the questionnaire (second study objective), the number of subjects with available data decreased with follow-up time. Missing responses on questionnaire items were replaced 
if, for a patient, the amount of missing values was $\leq 50 \%$ for a subscale. In case of $>50 \%$ missing values, the subject was excluded from further analysis of that particular subscale. Multiple imputation (MI) was used to generate 40 datasets. These datasets were then analyzed separately, and finally a single (pooled) $\mathrm{Ml}$ estimate and its standard error were calculated by combining the estimates and standard errors obtained from each completed dataset using "Rubin's rules" (Little \& Rubin, 2002; Rubin, 1987). The imputation method was determined by the "automatic" method in SPSS multiple imputation. Multilevel linear regression analyses with a compound symmetry covariance structure were performed to assess the course of the subscale sum scores and total sum score of the questionnaire over the different time periods. In case of multiple comparisons, $p$-values were again Bonferroni-Holm corrected, with alpha $\leq .05$ being considered statistically significant.

To evaluate the relation between results on the questionnaire and speech performance tests (third study aim), Spearman's correlation coefficients were calculated given the small sample size and since some variables did not display a normal distribution. Correlations were calculated between the difference scores ( 1 year post- vs. preoperative) on both the CNC word test and the ST-test on one side and the changes in outcome 1 year postimplantation on the total score and subscale scores of the questionnaire, on the other.

For the fourth study objective, the bivariate relations between preimplantation factors and the 1 year postoperative $\mathrm{CNC}$ word recognition score with $\mathrm{Cl}$, were first explored with simple linear regression. Multivariable regression analysis, using a backward method, was then performed with those variables that were significant in the simple linear regression analysis. During the backward regression analysis, non-significant preimplantation factors were sequentially removed (removal criterion: $p<.10$ ). In the final model there were then no more variables that met the removal criterion. The final model is presented with estimated coefficients, along with the "adjusted" R2, which is how much of the total variation in word recognition 1 year postoperative can be explained by the model, adjusted for the number of variables included. All analyses were performed using SPSS 22.0.

\section{RESULTS}

\section{Study objective 1: changes in auditory performance}

Figures $1 \mathrm{~A}, \mathrm{~B}$ and $\mathrm{C}$ show the individual and mean observed scores of the 27 subjects on the word recognition, sentence recognition and ST-tests, respectively. Multilevel analysis showed a significant effect of the time of measurement for word recognition $(F(4,19.41)=$ 5.139, $p=.005)$, sentence recognition $(F(4,21.89)=4.525, p=.008)$ and the ST-test $(F(4$, $78.31)=12.076, p<.0001)$. After Bonferroni-Holms' correction, pairwise comparisons 

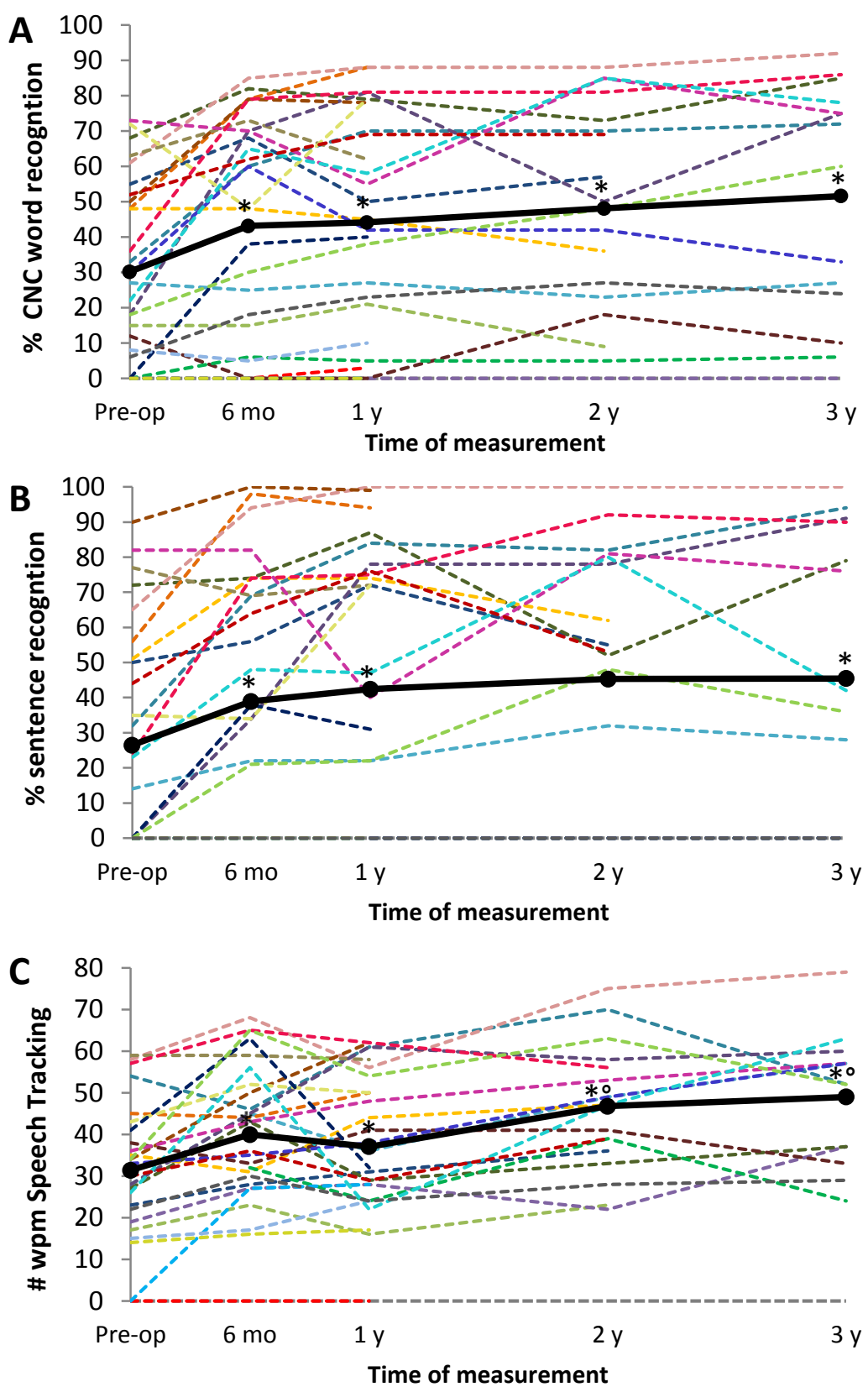

Figure 1. Individual (striped lines) and mean (thick line) observed scores on the CNC word recognition (A), sentence recognition (B) and ST-test (C) for $n=27$. Preoperative, 6 month, 1, 2 and 3 year postoperative observed mean scores were respectively 30.2, 43.2, 44.2, 48.1 and $51.6 \%$ for the CNC word scores (A); 26.5, 38.9, 42.4, 45.3 and $45.4 \%$ for the sentence recognition scores (B) and 31.4, 39.9, 37.0, 46.8 and 49 words per minute for the ST-test (C). For respectively 3, 10 and 1 subject(s), data points remain at 0 in figure A, B and C. Significant differences after Bonferroni-Holm's correction are indicated with * when compared to the preoperative situation and with ${ }^{\circ}$ when compared to the $1 y$ postoperative situation. 
revealed significantly higher scores for all postoperative measurements when compared to the preoperative situation, and this on all three tests, with the exception of the $2 y$ postoperative measurement of the sentence recognition test ( $p=.07$ after correction). On the ST-test, significant improvements were found 2 and 3 years postoperatively compared to the 1y postoperative situation. No further differences were found between the postoperative measurements on any of the tests.

Although postoperative improvements were found for the entire subject group, the scores of a number of subjects remained very low, for some even at $0 \%$. Table 2 shows the preoperative and 1 year postoperative scores of the individual subjects for the different outcome measures. The changes in score from pre-op to $1 \mathrm{y}$ post-op were added as difference scores. Subjects whose scores remained below $30 \%$ on the open-set word recognition test are labeled "poor performers" and marked with an asterisk in both Table 1 and Table 2. The $30 \%$ criterion applied was the same as that by Arisi et al. [2010] and Caposecco et al. [2012]. If the difference score of an individual subject in Table 2 was $\leq 5 \%$ / $\leq 5 \mathrm{wpm}$, the result was considered to be stable (no change). Inspection of individual scores reveals that only five subjects (S7, S12, S13, S18 and S22) show an improvement for all three tests, i.e., open-set word recognition, sentence recognition and speech tracking. Seven more subjects (S2, S5, S6, S8, S9, S25 and S26) show an improvement on two of the three tests, and have a stable score on the third. Subject 21 showed improvement on two tests, but there was no speech tracking score available. None of these in total 13 subjects belong to the group of earlier defined "poor performers". The remaining subjects who neither show improvements on at least two out of three tests nor belong to the poor performers are S11, S17, S19 and S20. The performance of both S11 and S19 is more or less the same before and after cochlear implantation. S11 performs well, both preoperatively with two hearing aids, as well as 1 year postoperatively with hearing aid and $\mathrm{Cl}$. S19 displays a small improvement on postoperative word recognition, while scores for sentence recognition and speech tracking remain unchanged. For S17 and S20 we see a discrepancy in their results, with improvements on two of the three tests, but declines on the third. When looking at the poor performers $(n=10)$, it can be seen that only S1 and S27 show an improvement on open-set word recognition, of 6 and 17\% respectively. S10, on the other hand, performs $12 \%$ worse postoperatively. For the remaining poor performers (S3, S4, S14, S15, S16, S23 and S24), phoneme scores on the open-set CNC word test remain stable. Only one of the poor performers (S3) improves more than $5 \%$ on the sentence recognition test and many obtain a $0 \%$ score, due to the test not being administrable. In contrast, scores on the speech tracking and particularly the MTS test show a much greater variation, with improvements for some subjects (S3, S4, S14, S15, S16, S23 and S27) and stable results or even slight declines for others (S1, S10 and S24).

In Figure 2, as well as at the bottom of Table 2, the mean observed scores of the 10 poor performing subjects are presented. S14 was excluded from the ST-test because there was 


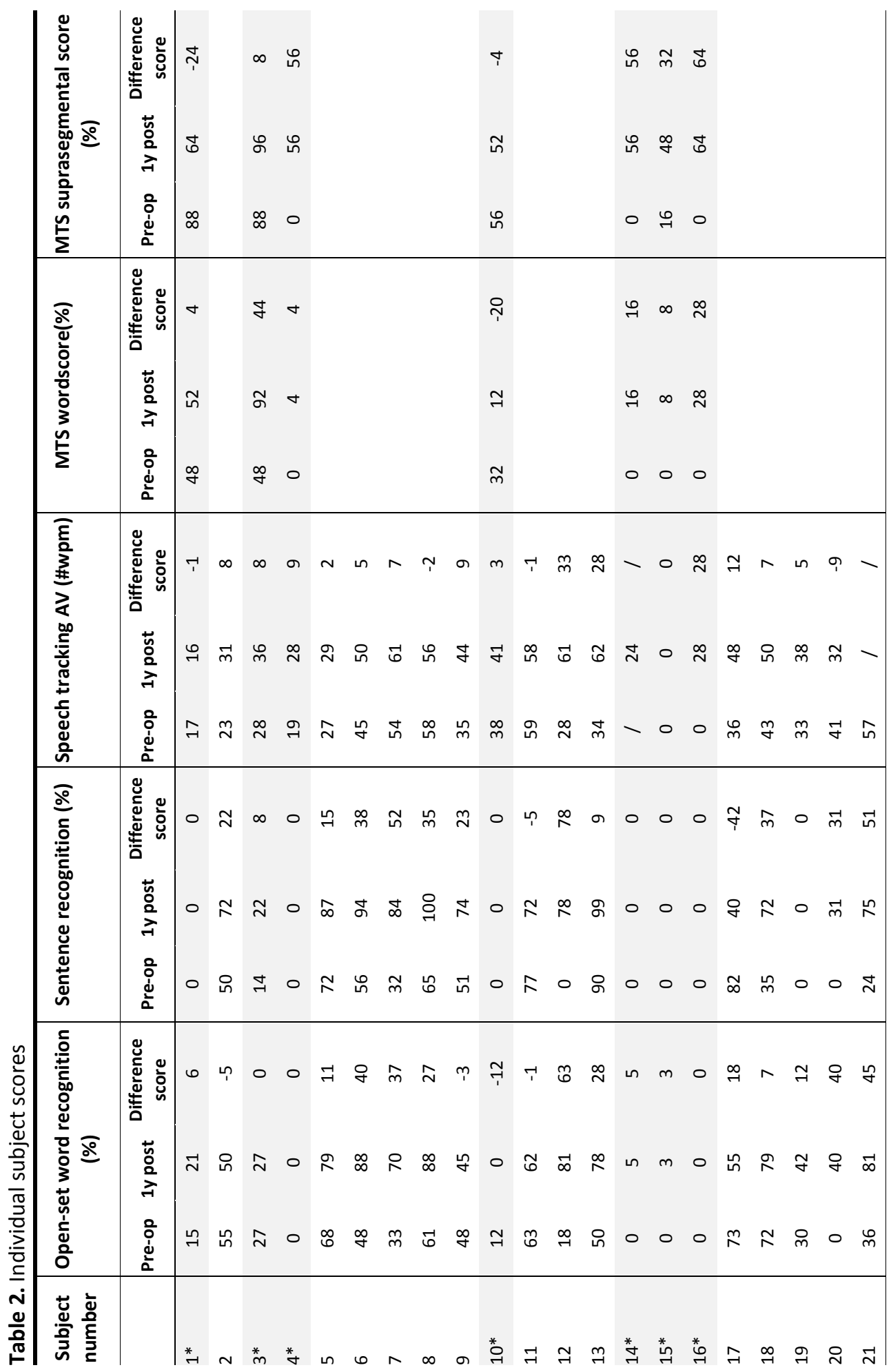


Chapter 6

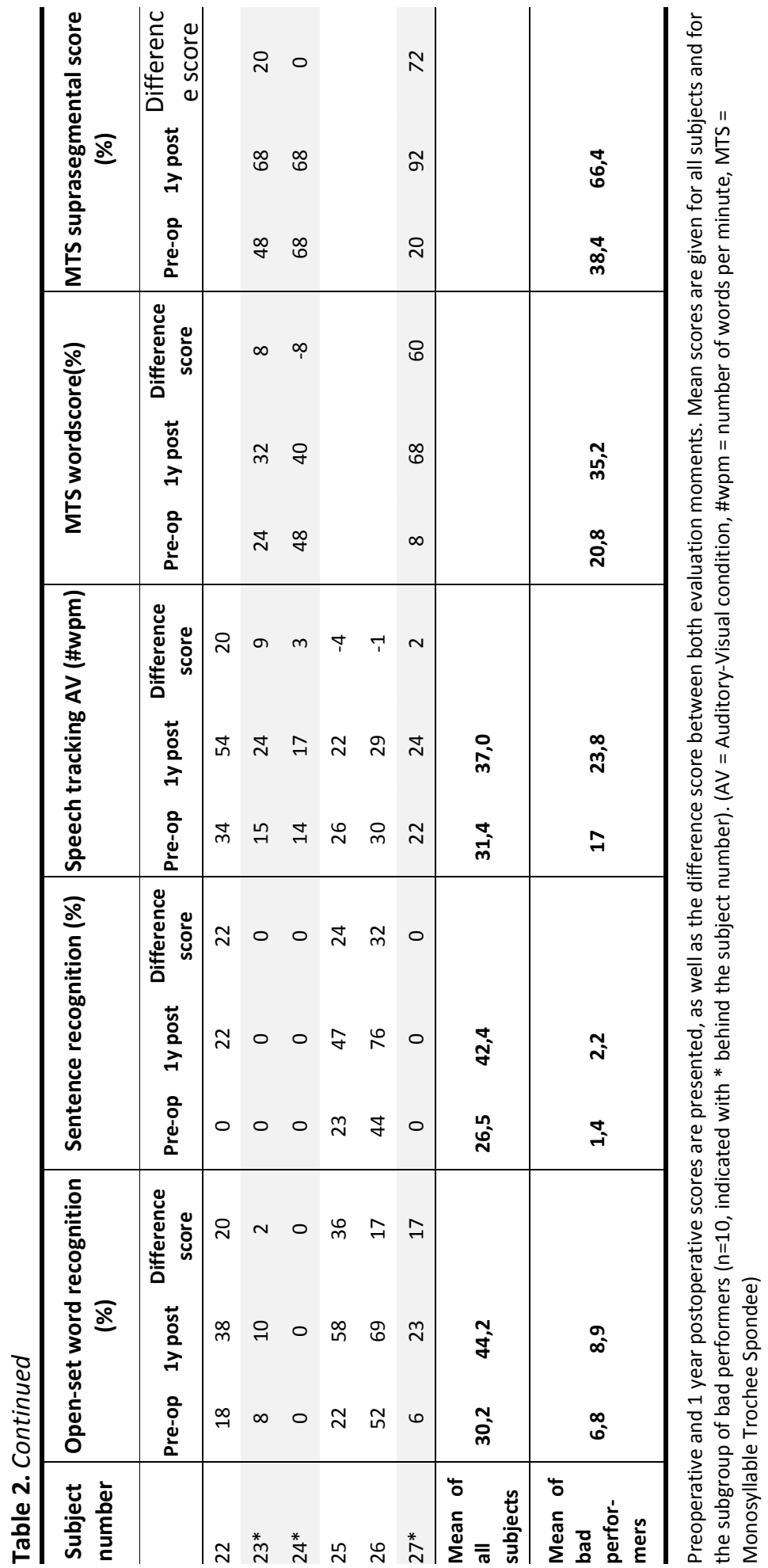




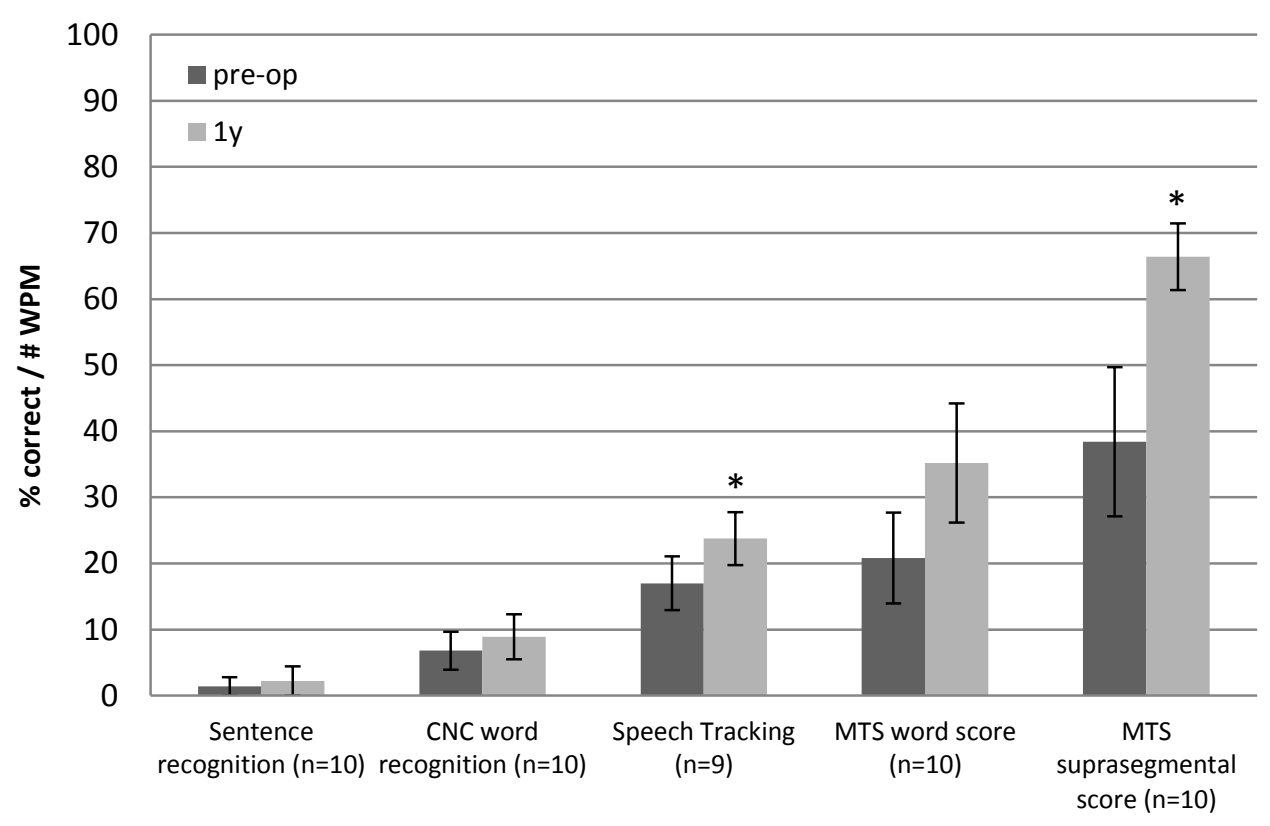

Figure 2. Preoperative and 1 year postoperative results of the poor performing group. Error bars represent the standard error of the mean. Asterisks $(*)$ indicate a significant difference compared to the preoperative situation.

no preoperative measurement. A statistically significant improvement from a mean pre-op score of 38.4 to a mean post-op score of $66.4 \%$ was found for the suprasegmental score on the MTS-test $(p=.038)$. For the word score on the MTS-test, the improvement from a mean pre-op score of $20.8 \%$ to $35.2 \%$ post-op was non-significant ( $p=.092$ ). On the STtest, a small but significant improvement from a mean of $17 \mathrm{wpm}$ pre-op to a mean of 23.8 wpm post-op $(p=.017)$ was seen. There was no improvement in the mean open-set word and sentence recognition scores after 1 year of $\mathrm{Cl}$ use.

\section{Study objective 2: self-perceived benefits}

Questionnaire scale scores were obtained at pre- and 1 year postoperative for 16 to 20 subjects (depending on the subscale) and at 2 and 3 year postoperative for 13-14, respectively, 11 subjects (see Figure 3 ). Multilevel analysis found significant improvements at $1 y$ postoperative compared to preoperative for the total questionnaire score and each of the separate subscales except for "social aspects of hearing". The largest mean improvements were seen for primary sound processing (from $47.4 \%$ pre-op to $69.4 \% 1 \mathrm{y}$ post) and sense of safety (from $40.8 \%$ pre-op to $62.4 \% 1 \mathrm{y}$ post); the total score improved from $53.2 \%$ preoperatively to $63.9 \% 1$ year postoperatively. On the long term, scores remained significantly higher than preoperatively for the total questionnaire and for the subscales "primary sound processing" and "ease of communication". This was not the case for "self-confidence" (at 2 and 3y post-op) and "sense of safety" (at 3y post-op). Total 


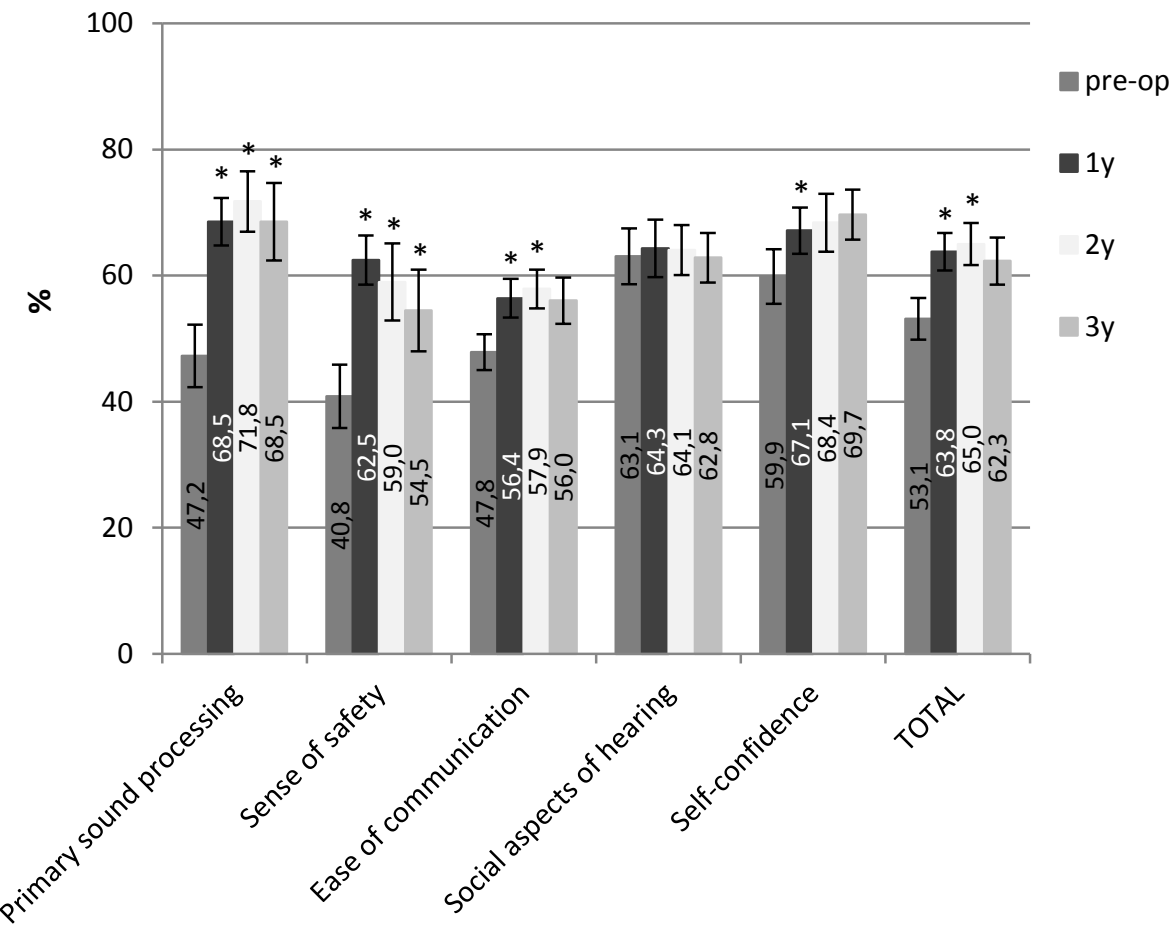

Figure 3. Mean observed scores (expressed in \%) on the questionnaire for the 5 separate subscales and the total questionnaire. Error bars represent the standard error of the mean. $\mathrm{N}$ is 16 to 20 for the pre-op and $1 \mathrm{y}$ post-op measurement, 13 to 14 for the $2 y$ post-op measurement and 11 for the 3y post-op measurement. Asterisks $\left({ }^{*}\right)$ indicate a significant difference after Bonferroni-Holms' correction, compared to the preoperative situation.

questionnaire scores were available for five of the 10 poor performing subjects and showed a significant improvement of almost $11 \% 1$ year post-op $(z=-2.023, p=.043)$.

\section{Study objective 3: relations between auditory and self-perceived benefits}

Correlation analyses of the changes in auditory performance with the changes on the questionnaire 1 year postoperatively, revealed very weak to weak, nonsignificant correlations. Spearman correlations between the questionnaire (subscales and total questionnaire) and the difference scores on open-set word recognition are positive and range between .09 and .38 ( $n=16$ to 20$)$; correlations between difference scores on the questionnaire and ST performance are close to zero (range: -.08 and $.12, n=14$ to 20 ).

\section{Study objective 4: predictive value of preimplantation factors on speech recognition scores}

Simple regression analysis indicated that six of the eight preimplantation factors were significant predictors for the 1 year postoperative CNC word recognition scores (left side of Table 3). Inclusion of these six factors as independent variables in a backward 
Table 3. Regression analyses

\begin{tabular}{lcccc} 
& \multicolumn{3}{c}{ Simple regression } \\
& B & SE B & $\boldsymbol{\beta}$ & $\boldsymbol{p}$ \\
Pre-op PTA (better ear) & -1.396 & 0.368 & -0.605 & .000 \\
Pre-op PTA (implanted ear) & -2.145 & 0.379 & -0.750 & .000 \\
Pre-op CNC word recognition (best-aided) & 0.759 & 0.162 & 0.685 & .000 \\
Pre-op CNC word recognition (implanted ear) & 0.828 & 0.205 & 0.629 & .000 \\
Hearing aid use at implantation: yes/no & -38.957 & 13.416 & -0.502 & .004 \\
Hearing aid use at implantation (implanted ear): yes/no & -37.278 & 9.006 & -0.638 & .000 \\
Pre vs. perilingual onset of deafness & 3.945 & 14.167 & 0.056 & .391 \\
Communication mode at implantation: Oral vs. Oral + manual & -18.026 & 11.519 & -0.299 & .065 \\
\hline & \multicolumn{2}{c}{ Multivariable regression } \\
& B & SE B & $\boldsymbol{\beta}$ & $\boldsymbol{p}$ \\
\hline & NS & & & \\
Pre-op PTA (better ear) & -1.670 & 0.382 & -0.584 & .000 \\
Pre-op PTA (implanted ear) & NS & & \\
Pre-op CNC word recognition (best-aided) & 0.472 & 0.176 & 0.359 \\
Pre-op CNC word recognition (implanted ear) & NS & & \\
Hearing aid use at implantation: yes/no & NS & & \\
Hearing aid use at implantation (implanted ear): yes/no & & &
\end{tabular}

Values for the unstandardized regression coefficient (B), standard error (SE) of $B$, standardized regression coefficient Beta $(\beta)$ and the significance values $p$ are given for the regression of CNC word recognition score with $\mathrm{Cl}$ only, 1y post implantation, on 8 investigated preimplantation factors. For the simple regression the standardized coefficients $\beta$ represent the correlations between the preimplantation factors and the outcome variable. For the multivariable regression, two preimplantation factors remained as significant predictors in the final model after backward analysis. The adjusted $\mathrm{R}^{2}$ for the final model was .635. (PTA= Pure Tone Average at 0,$5 ; 1 ; 2 \mathrm{kHz}, \mathrm{NS}=$ Not Signficant), $\mathrm{n}=27$

regression procedure resulted in a model including preoperative PTA $(p<.0001)$ and preoperative CNC word recognition score $(p=.013)$, both for the implanted ear, as predictors of the CNC word recognition score with $\mathrm{Cl} 1$ year postoperative (right side of Table 3) with an explained variation of $63.5 \%$. The backward analyses performed are presented in Appendix C.

\section{DISCUSSION}

\section{Study objective 1: changes in auditory performance}

The first aim of this study was to evaluate the postoperative change in auditory performance of a group of early-deafened, late implanted adult $\mathrm{Cl}$ users by means of both traditional (open-set word and sentence recognition) and non-traditional (speech tracking (ST) and the monosyllable-trochee-spondee test (MTS)) tests, administered in the bestaided condition. A limitation concerning both the sentence recognition and ST-test, is that they are not validated. Results of the multilevel analysis showed a significant effect of the time of measurement for all three tests. Due to the relatively small number of subjects, which moreover decreased with follow-up time, these results do need to be interpreted with caution. Compared to the pre-operative situation, significant improvements were present already after 6 months of $\mathrm{Cl}$ use. The only exception was the 2 year postoperative 
measurement of the sentence recognition test, which lost its statistical significance after correction for multiple comparisons. Since no drop in performance was observed 2 year postoperatively (see Figure 1B), this lack of statistical significance might be explained by the reduction in statistical power due to a smaller sample size for that measurement. The fact that the change 3 years postoperatively once again attained statistical significance, even though the observed score remained the same with an even smaller sample size, is presumably due to the higher estimation of the mean, as predicted by the multilevel model. The mean word recognition scores of $44.1 \%$ (range $0-88 \%$ ) and mean sentence recognition scores of $42.4 \%$ (range $0-100 \%$ ) at the 1 year postoperative measurement are comparable to those found in other studies, although it is unclear for some of these studies whether the reported scores were obtained with $\mathrm{Cl}$ only or in the best aided condition (Caposecco et al., 2012; Klop et al., 2007; Santarelli et al., 2008; van Dijkhuizen et al., 2011; Yang et al., 2011; Zeitler et al., 2012). Consistent with literature, we found extremely large differences between individual word and sentence recognition scores (see Figures $1 \mathrm{~A}$ and $1 \mathrm{~B})$.

Until now, there has been no literature reporting speech tracking for this patient group. Besides being administered in the best-aided condition, the ST-test was performed with the aid of visual cues, bringing it more in line with everyday life communication. Although the absolute improvements were small for most subjects, the improvement was significant at the group level. Given that most of the subjects in this study were excellent lip readers, these apparently small changes are most likely explained by the fact that the added value of the $\mathrm{Cl}$ becomes apparent in conjunction with the best communicative situation (e.g. lip reading). Results therefore show that it can be demonstrated that cochlear implantation can improve communication speed, when measured in a test situation that is as close to real life communication as possible.

Another part of our first study objective was to see how performance on various tests evolved in the course of time. It is clear from Figure 1 that the largest changes occurred from pre-op to 6 months post-op, for all three tests. No significant improvements were found thereafter, except for $2 y$ and $3 y$ post-op versus $1 y$ post-op on the ST-test (fig. 1C), although these may be attributable to the small decrease in performance 1 year postoperatively. Our results therefore conform to those on postlingual adults and do not support the hypothesis purported by Santarelli et al. (2008) that prelingually deafened $\mathrm{Cl}$ users might show improved results in the long-term due to remaining plasticity in the auditory cortical areas.

Descriptive analysis of the individual pre- and 1y postoperative scores (Table 2) show that, of the 17 subjects that obtained more than 30\% open-set word recognition 1 year post implantation, 13 showed a clear improvement on at least two out of the three tests including open-set word recognition, sentence recognition and speech tracking. Two subjects (S11 and S19) scored the same before and after implantation, and two (S17 and S20) had mixed results, for which we have no explanation. On the other hand, the 10 
subjects that scored below 30\% word recognition 1y post-op, generally showed no improvement on open-set word or sentence recognition. One could draw the conclusion that there is no measurable auditory benefit of cochlear implantation in these subjects. However, when taking the results of the non-traditional outcome measures - speech tracking and particularly MTS - into account, new information is added which allows us to distinguish between subjects that do show a measurable benefit with their $\mathrm{Cl}$ and those who don't. Some subjects who are considered to be unsuccessful $\mathrm{Cl}$ users do obtain auditory benefits. While no significant improvements were found for the open-set word and sentence recognition test, the suprasegmental score on the closed-set MTS-test and the number of correctly repeated words per minute on the ST-test, did show significant improvements. Our hypothesis, that non-traditional auditory measures are more suitable than traditional open-set, auditory-only speech tests to detect true auditory gains in poor performing subjects, is thus confirmed. It can be therefore be advised to gear the choice of speech tests to the performance level of the subject, considering both standard and less traditional tests for an optimal monitoring of outcomes.

It is interesting to note that all four subjects who did not wear hearing aids preoperatively, belong to the poor performing group. These subjects did wear hearing aids during childhood but stopped wearing them as adolescents, presumably due to limited benefit. However, if we further compare the characteristics of those poor performing subjects that show no postoperative improvement on any of the tests with those that do, there are no clear differences to be found in terms of communication mode, age at implantation or hearing aid use.

\section{Study objective 2: self-perceived benefits}

Subjective experience after $\mathrm{Cl}$ should be an integral part of the evaluation protocol. A limitation of the questionnaire used in this study, which was specifically designed for the early-deafened group of $\mathrm{Cl}$ users, is of course the lack of sufficient validation. Results on the questionnaire revealed that subjective scores were significantly higher postimplantation for the subscales "primary sound processing", "sense of safety" and "ease of communication". Other studies that assessed hearing-related quality of life before and after implantation in this patient group also found significant postoperative improvements when using the NCIQ (Klop et al., 2007; Straatman et al., 2014; van Dijkhuizen et al., 2011), PIPSL (Schramm et al., 2002) or a number of self-reporting questionnaires (Most et al., 2010). In our study there was no effect of the $\mathrm{Cl}$ on the subscale "social aspects of hearing", and the effect on "self-confidence" was only significant at 1y post-op. The subscale "social aspects of hearing" contains questions like "Do you like to go to parties or meetings?" and "How often do you feel left out by others?". A likely explanation for the absence of a significant change in this subscale may be the early onset of deafness. The subjects in this study had been participating in social situations with a severe hearing impairment for their entire lives, which is also illustrated by the relatively high pre-op 
scores in this subscale so that noticeable changes in this area are unlikely. The same reasoning may explain the slight changes found in the subscale "self-confidence". Other studies failed to find significant postoperative improvements for subscales related to selfconfidence or self-esteem (Klop et al., 2007; Most et al., 2010), or found slightly smaller changes (Straatman et al., 2014). Although it is hard to compare the content of the different questionnaires, it makes sense that the subscale "self-esteem" of the NCIQ also encompasses questions that are related to the subscale "social aspects of hearing" of the current study.

\section{Study objective 3: relations between auditory and self-perceived benefits}

Auditory gains on either CNC word recognition or speech tracking did not appear to be related to benefit scores on the questionnaire. This might in part be attributable to the fact that there were significant improvements in the questionnaire scores for the subgroup of poor performers. Our results are in agreement with Straatman et al. (2014), although van Dijkhuizen et al. (2011) did find some significant correlations, but only with the subscale "advanced sound perception" of the NCIQ. This appears to indicate that in early-deafened $\mathrm{Cl}$ users, quality of life benefit scores are generally independent of auditory gains. When judging whether an implantation is successful, both types of outcome measures should thus be taken into account, since they evaluate distinctly different aspects of cochlear implantation rehabilitation. A so-called "poor" performer might experience subjective benefits without improvement on open-set speech tests. Also, the fact that a correlation with the advancements on the speech tracking test was not found, which - in contrast to the open-set word test - did show postoperative benefits even in the poor performing group, suggests that self-perceived changes are not related to the improvements in day-to-day communication skills. It would be interesting to investigate further the relation between subjective outcomes and, for instance, the MTStest in a larger group of poor performers.

\section{Study objective 4: predictive value of preimplantation factors on speech recognition scores}

Two of the eight investigated preimplantation factors remained in the final multiple regression model: the preoperative CNC word recognition score and the preoperative PTA, both for the implanted ear, together explaining $63.5 \%$ of the variation in postoperative speech understanding with $\mathrm{Cl}$. This result needs to be interpreted with caution however, given that the regression analyses were based on data of only 27 subjects. Two recent studies also performed multiple regression analysis in groups of respectively 58 and 43 early-deafened subjects (Kraaijenga et al., 2016; Rousset et al., 2016). In the study by Kraaijenga et al. (2016), the preoperative best-aided word recognition score, and not the score of the ear to be implanted as in our study, was found to be the only significant predictor of the $1 y$ post-op word recognition scores, and explained $31 \%$ of the variation. In that same study, the preoperative PTA of the $\mathrm{Cl}$-ear, which was the second significant 
factor in our model, correlated significantly with the outcome variable but lost its significance in the subsequent multivariable analysis. In the study by Rousset et al. (2016), the preoperative phoneme score of the ear to be implanted could, together with a standard score for receptive language abilities, account for $26.3 \%$ of the variation in postoperative speech perception scores. In their study, the PTA of the implanted ear, however, did not have a significant correlation with the outcome measure. Given that the word recognition score of the implanted ear was a significant predictor in both Rousset et al. (2016) as in the current study, it seems worthwhile to include this factor in future studies.

While only two preimplantation factors remained as significant predictors in our final model, there were four more preimplantation factors correlating significantly with the outcome measure: preoperative best-aided CNC word recognition, preoperative PTA of the better ear, hearing aid use at implantation (yes/no) and hearing aid use at implantation in the implanted ear (yes/no). As mentioned previously, the preoperative best-aided word recognition score was a significant predictor in the multivariable regression model of Kraaijenga et al. (2016). van der Marel et al. (2015) equally observed a significant correlation with preoperative phoneme scores. On the other hand, van Dijkhuizen et al. (2016) (after Bonferroni correction) and Zeitler et al. (2012) did not find a significant correlation with preoperative CNC scores. Similarly to the preoperative CNC scores, our study found the preoperative PTA of the implanted ear to be a significant predictor, whereas the significant correlation of the preoperative PTA of the better ear was dropped in the backward multiple regression modeling procedure. Both preimplantation factors regarding hearing aid use at implantation, were also dropped in the backward procedure. In 2012, Caposecco et al. found that the time without an aid in the implant ear (at implantation) was a significant predictor, while van Dijkhuizen et al. (2016) found no significant correlation between postoperative speech perception and hearing aid use.

Finally, two of the eight preimplantation factors did not show a significant correlation with our outcome measure: communication mode at implantation and pre- vs. perilingual onset of deafness. The first is somewhat surprising, given that advantages for oral communicators are seen in most (Caposecco et al., 2012; Rousset et al., 2016; Yang et al., 2011; Zeitler et al., 2012), yet not all previous studies (van Dijkhuizen et al., 2016). The factor regarding pre- vs. perilingual onset of deafness has only been assessed by Caposecco et al. (2012). In contrast to our results, this study did find a significant relation between the progressivity of the hearing loss and sentence recognition scores with $\mathrm{Cl}$.

\section{CONCLUSION}

This study showed that early-deafened but late implanted adult $\mathrm{Cl}$ users obtained significant auditory improvements, mainly within the first six months after implantation. It 


\section{Chapter 6}

was found that, on average, auditory performance of poor performing subjects increases but this can only be captured by using specific tests like speech tracking or tests assessing more suprasegmental features of speech. On an individual subject level, these new outcome measures add valuable information and allow for more differentiation between poor performing subjects. The current results emphasize the importance of using a different test battery for the auditory evaluation of this group of $\mathrm{Cl}$ users.

An instrument assessing subjective experiences after $\mathrm{Cl}$ should be an integral component of the evaluation protocol, given that individually experienced benefit is not fully captured by the auditory tests.

Postoperative performance with $\mathrm{Cl}$ could be explained to a large part by two preimplantation factors: preoperative PTA and preoperative CNC word recognition, both of the implanted ear. Studies in larger groups of early-deafened patients could contribute further to understanding the role that preimplantation factors have on $\mathrm{Cl}$ outcome in this population. 


\section{APPENDIX A: Reliability analysis of the subjective questionnaire}

As a first step in the development of the questionnaire used in this study, structured interviews using open questions were performed with a number of early-deafened $\mathrm{Cl}$ users, to identify the domains on which these $\mathrm{Cl}$ users experienced changes after their implantation. These findings were used to formulate questions covering aspects of hearing and communication, social and psychological functioning. Questions were intuitively divided in five subscales: primary sound processing, sense of safety, ease of communication, social aspects of hearing and self-confidence. The original questionnaire contained 55 questions, with a 5-point Likert response scale for each question. For computation of the scores on the questions, the answer categories were transformed, with never/not at all $=0$, occasionally $/$ somewhat $=25$, regularly $/$ more or less $=50$, usually/for the most part $=75$ and always/completely $=100$. For 20 questions which were phrased in opposite form (questions number $3,4,5,8,9,14,15,16,18,25,27,29,31,36$, $37,40,43,44,45,47$ ), the transformation was reversed.

The results of the 20 subjects that completed the questionnaire 1 year postoperatively were used to perform a reliability analysis. First, three questions which had more than $15 \%$ missing values (left blank or "not applicable"), were considered not to be relevant for this patient population and were left out in further analysis. Subjects that had missing values for more than $25 \%$ of the questions in a certain subscale were equally excluded from the reliability analysis of that particular subscale. Remaining missing values were imputed 40 times for each subscale, using multiple imputation, presupposing the missing mechanism is Missing At Random (MAR), which means that the probability of an observation being missing does not depend on unobserved measurements. Cronbach's alpha was calculated for each of the 40 imputed datasets. Questions that, if left out, increased the average Cronbach's alpha over the 40 data sets by at least .02, were excluded from that subscale of the questionnaire. This resulted in a final questionnaire containing 50 questions in five subscales: primary sound processing (8 questions), sense of safety (5 questions), ease of communication (12 questions), social aspects of hearing (12 questions) and self-confidence (14 questions). Values of Cronbach's alpha were .83, .57, $.77, .92$ and .86 respectively. The final questionnaire can be found in Appendix B. 
Chapter 6

APPENDIX B: Subjective questionnaire for early-deafened $\mathrm{Cl}$ users (translated from Dutch by a native English speaker)

Never Occasionally Regularly Usually Always N/A

1. Can you hear soft

sounds (such as

running water,

twittering birds, the

clock ticking) in quiet

surroundings?

2. Is it easy for you to express your own opinion?

3. Do you have to concentrate to follow a conversation?

4. Do you feel ignored by others?

5. Are you sometimes relieved when a conversation is over?

6. Can you recognize everyday sounds in your home?

7. Do you like to go to parties or meetings?

8. Do you feel like you are worth less than other people?

9. Do you sometimes need to have things repeated in a conversation?

10. Are you happy about the things you can do and what you actually do?

11. Can you hear the phone ringing in the same room?

12. Are colleagues and acquaintances considerate towards you? 
13. Are strangers able to

Never Occasionally Regularly Usually Always N/A

understand what you

are saying?

14. Are you nervous when you need to speak in a group?

15. How often do you feel alone?

16. Do conversations sometimes end soon because they are tiresome?

17. Can you hear cars and motorbikes approaching when you are outside?

18. Are you embarrassed by your hearing problems?

19. Are you at ease in a group?

20. Can you hear the difference between clinking silverware and clapping hands?

21. Are you able to perform your daily chores/work?

22. Is it easy for you to initiate a conversation with someone you do not know well?

23. Can you hear the doorbell?

24. Is it easy for you to meet new people?

25. Do you try to hide your hearing problems?

26. Can you hear the difference between the voice of a man and a child? 
Chapter 6

Never Occasionally Regularly Usually Always N/A

27. How often do you

feel depressed?

28. Do you like being in a group of people?

29. Do

misunderstandings

sometimes occur

during conversation?

30. Can you hear

someone calling your

name from another

room?

31. Does it bother you

that you are

deaf/hearing

impaired?

32. Do friends and family show consideration for you?

33. Can you recognize the voice of someone you know well in a quiet room?

34. Do you think you are successful?

35. Can you hear the voice of the person next to you in a quiet room?

36. Is it embarrassing for you to have to say something in a group?

37. How often are you tense or tired at the end of the day?

38. Do you think your opinion is important?

39. Can you hear the difference between music and the news on the radio?

40. How often do you feel stressful? 
41. Can you hear

Never Occasionally Regularly Usually Always N/A

someone calling your name in a quiet room?

42. Do you like to talk to others?

43. Are you worried that others cannot understand you?

44. How often do you feel left out by others?

45. Do you need to read lips to understand someone?

46. Can you hear whether a car is far away or close by?

47. Does it bother you when you cannot understand someone?

48. Can you hear loud sounds (such as traffic, a dog barking, door slamming,...)?

! The answer categories of Not Somewhat More or
less For the Completely N/A the following 2 questions at all most are different! part

49. You are talking to one person in a quiet room. You can see that person's lips. Can you understand this person?

50. You are talking to one person at a party or meeting (busy surroundings). You can see that person's lips. Can you understand this person? 
Chapter 6

APPENDIX C: Overview of the backward multivariable regression analysis

\begin{tabular}{|c|c|c|c|c|}
\hline Preimplantation factors & B & SE B & $\boldsymbol{\beta}$ & $p$ \\
\hline \multicolumn{5}{|l|}{ Model 1} \\
\hline Pre-op PTA (better ear) & -0.535 & 0.709 & -0.232 & .460 \\
\hline Pre-op PTA (implanted ear) & -0.982 & 0.934 & -0.343 & .305 \\
\hline Pre-op CNC word recognition (best-aided) & -0.129 & 0.318 & -0.116 & .690 \\
\hline Pre-op CNC word recognition (implanted ear) & 0.561 & 0.308 & 0.426 & .084 \\
\hline Hearing aid use at implantation: yes/no & -10.022 & 13.150 & -0.129 & .455 \\
\hline $\begin{array}{l}\text { Hearing aid use at implantation (implanted ear): } \\
\text { yes/no }\end{array}$ & -3.423 & 12.113 & -0.059 & .780 \\
\hline \multicolumn{5}{|l|}{ Model 2} \\
\hline Pre-op PTA (better ear) & -0.564 & 0.686 & -0.244 & .420 \\
\hline Pre-op PTA (implanted ear) & -1.052 & 0.880 & -0.368 & .245 \\
\hline Pre-op CNC word recognition (best-aided) & -0.165 & 0.285 & -0.148 & .570 \\
\hline Pre-op CNC word recognition (implanted ear) & 0.604 & 0.263 & 0.459 & .032 \\
\hline Hearing aid use at implantation: yes/no & -11.831 & 11.231 & -0.153 & .304 \\
\hline \multicolumn{5}{|l|}{ Model 3} \\
\hline Pre-op PTA (better ear) & -0.399 & 0.614 & -0.173 & .522 \\
\hline Pre-op PTA (implanted ear) & -1.019 & 0.865 & -0.356 & .251 \\
\hline Pre-op CNC word recognition (implanted ear) & 0.509 & 0.202 & 0.387 & .020 \\
\hline Hearing aid use at implantation: yes/no & -10.411 & 10.792 & -0.134 & .345 \\
\hline \multicolumn{5}{|l|}{ Model 4} \\
\hline Pre-op PTA (implanted ear) & -1.508 & 0.418 & -0.527 & .001 \\
\hline Pre-op CNC word recognition (implanted ear) & 0.449 & 0.178 & 0.341 & .019 \\
\hline Hearing aid use at implantation: yes/no & -10.203 & 10.651 & -0.132 & .348 \\
\hline \multicolumn{5}{|l|}{ Model 5} \\
\hline Pre-op PTA (implanted ear) & -1.670 & 0.382 & -0.584 & .000 \\
\hline Pre-op CNC word recognition (implanted ear) & 0.472 & 0.176 & 0.359 & .013 \\
\hline
\end{tabular}

Adjusted $\mathrm{R}^{2}=.595$ for Model 1, .613 for Model 2, .625 for Model 3, .634 for Model 4 and .635 for Model 5 (= final model) 
Chapter 7

General discussion and valorisation 

The aim of the current thesis was to expand current knowledge regarding cochlear implantation in a very specific patient group: adults or adolescents with an onset of deafness in early childhood. In the different chapters of this thesis we presented data on the outcomes of this patient group with $\mathrm{Cl}$ and on the factors that might influence these outcomes, either patient-related or related to the fitting and coding of the electrical signal.

\section{Defining the target group}

One of the difficulties we came across in the course of this thesis was how to define and demarcate our specific target group of patients. In the different chapters they are referred to as congenitally, prelingually, perilingually, as well as early-deaf(ened) patients. Where the first three terms each refer to a different age at onset, "early" is a more comprehensive term, generally referring to any age at onset before the end of the language acquisition period. Although the term "prelingual deafness" has been used quite broadly in literature to indicate an onset before the age of 4 or even 6 years (Santarelli et al., 2008; Teoh et al., 2004a; van Dijkhuizen et al., 2016), and has also been used in that sense in chapters 2 and 3 of this thesis, it is actually defined as an onset before the beginning of the language acquisition period.

The differentiation of patients based on age at onset is important, as it is presumed that even minimal auditory exposure affects auditory development compared to no auditory exposure at all (Kral, 2007), but at the same time it is practically unfeasible as the medical and audiological information required to make such a differentiation is usually lacking, especially in patients born before the introduction of new born hearing screening. Even the clinical classification of a patient as "early" deaf, the term we adopted in chapters 4, 5 and 6 , is mostly based on the combined information obtained from a number of sources such as available medical files and audiograms from the past, presence of deaf speech, type of education received, and recall of family members on the onset of the hearing impairment.

An important aspect adding further variability to our target group is that not only the age, but also the degree and configuration of the hearing loss are often unknown for patients born several decades ago. Taken together, our target group of early-deafened patients is necessarily diverse, the use of strict definitions being unfeasible, primarily due to the lack of sufficient information on the hearing history of many patients.

From another angle, the demarcation of our target group is equally determined by the age at which cochlear implantation is performed. The main issue is whether subjects implanted as adolescents or adults, with a comparable onset of deafness, can be seen as one group or not. From what implantation age onwards is it reasonable to say that the influence of the early onset of the hearing impairment outweighs the influence of the age at implantation? Is there for example a difference between early-deafened subjects 
implanted at age 12 vs. 25, based on their age at implantation? In the review in chapter 5 , we concluded that age at implantation is very likely not related to $\mathrm{Cl}$ outcomes in adults with early onset of deafness, whereas in adolescents this relation does need further investigation. This implies that, until this is further clarified, the better option would be to separately analyse outcomes of early-deafened adolescents and adults if the aim is to see how these subjects perform as a group. This is also the case in chapters 2, 3 and 6 of this thesis, where all subjects were implanted after the age of 18 years. On the other hand, based on evidence from N1 and P1 potentials, Sharma et al. (2015) showed that "in all likelihood, the sensitive period ends by age 7 years, resulting in a re-organized auditory cortex that is unable to effectively process the stimulation provided by the cochlear implant". The reasons why the outcome of $\mathrm{Cl}$ would be different for adolescents and adults, as sensitive periods are closed in both cases, is an additional question that would then have to be addressed. Potentially other factors such as education and early hearing aid rehabilitation could explain the difference, rather than the younger age itself.

\section{Optimizing fitting parameters}

Chapters 2 and 3 of this thesis revolve around how technical aspects of cochlear implant signal processing, such as fitting parameters and properties of the $\mathrm{Cl}$ signal, might affect outcomes in our target group of $\mathrm{Cl}$ users. Current cochlear implant processing generally employs a "one size fits all" approach with respect to most fitting parameters, such as stimulation rate, number of stimulated electrodes, frequency allocation, etc. Although these default settings work well for most postlingual $\mathrm{Cl}$ users, they are not necessarily optimal for early-deafened $\mathrm{Cl}$ users who have developed a completely different auditory system due to their early onset of auditory deprivation. We therefore hypothesized that a more individualised approach towards fitting could improve performance, with psychophysical measures of certain signal processing aspects being used to change fitting parameters.

In chapter 2 we measured amplitude modulation detection thresholds (AMDTs) to assess temporal processing abilities of both pre- and postlingually deafened $\mathrm{Cl}$ users. This method is commonly used since the $\mathrm{Cl}$ processor equally applies amplitude modulations to the envelope of the fast electrical pulse trains that are delivered to the electrodes. We were able to demonstrate that the amplitude modulation detection abilities of the earlydeafened group were significantly poorer than those of the postlingually deafened group, especially for fast modulations. In addition, AMDTs were significantly correlated with speech recognition measures, also for the early-deafened subjects. If this relation were to be a causal one, a better detection of amplitude variations over time could potentially lead to better speech understanding. Consequently, it would be interesting to investigate whether improving the transmission of these temporal cues by means of changes to the speech processing strategy, could lead to improved speech recognition. So far, however, 
research efforts focusing on the effects of changing stimulation rate and/or stimulation level, which both influence the transmission of temporal cues, have not been successful at finding consistent and significant improvements on temporal or speech processing abilities (overview in Brochier, McDermott, and McKay (2017)). Since these studies are generally performed in postlingually deafened $\mathrm{Cl}$ users, and we found that temporal processing was significantly poorer for the average early-deafened $\mathrm{Cl}$ user, it would be interesting for future studies to investigate these relations specifically in an earlydeafened subject group. In addition, as our study showed that the ability to detect slow modulations was related to speech understanding performance in this group, those subjects having most difficulty detecting these slow modulations are also the ones for which it would be most interesting to investigate the effect of changing these parameters. Where chapter 2 focuses on the processing of temporal cues, chapter 3 gives attention to the coding of spectral parameters, related to the place of stimulation. In chapter 3 we first measured spectral processing through the ability to perform electrode discrimination, and then attempted to optimize the processing of spectral cues by means of deactivating all non-discriminable electrodes. A clear advantage of the adapted fitting on outcomes could not be found, however. In addition to the limited number of subjects, the influence of a number of other parameters that were changed along with the deactivation of electrodes could not be ruled out, and gives rise to further investigation.

In chapter 3, the limited number of subjects unfortunately did not allow for an investigation of the relation between spectral discrimination performance and speech understanding outcomes with $\mathrm{Cl}$, but other studies have been able to demonstrate such a relation, also in prelingually deafened adults (Busby \& Clark, 2000; Gifford et al., 2018). The presence of such a relation suggests that an optimization of the coding of spectral cues could have a positive impact on speech understanding outcomes as well. The success of studies, including our own, attempting to improve spectral resolution by means of deactivating electrodes that poorly encode speech information, is however mixed (Henshall \& McKay, 2001; Saleh et al., 2013; Vickers, Degun, Canas, Stainsby, \& Vanpoucke, 2016; Zwolan et al., 1997).

A recent study by Sagi and Svirsky (2018) offers new explanations for why purely removing the poorest encoding electrodes from the fitting map might not be the best solution. By means of extensive mathematical modelling, they were able to demonstrate that the main limiting factor in previous studies is the fact that the criteria used to deactivate the poorest encoding electrodes do not take the distribution of speech information over the remaining electrodes into account. Their results show that the benefit in speech recognition was significantly greater when an electrode deactivation pattern was used that maximizes the discrimination of speech cues ("model-optimized"), compared to a deactivation pattern that simply removes the poorest encoding electrodes ("best electrodes"). For the poorer performing group in this study (electrode discrimination difference limens between 0 and 4 electrodes), which is most representative for our study 
group of early-deafened $\mathrm{Cl}$ users, maximum improvements were demonstrated for the model-optimized approach when 11 out of the 22 electrodes remained active.

A recurring bottleneck, both in our study (chapter 3) as in the model of Sagi and Svirsky (2018), is that complete adaptation to each new combination of electrodes is required in order to attain a maximal score for this combination. Presuming however, that subjects are able to completely adapt given sufficient time, these new insights demonstrate that combining a measure for identifying poorly discriminating electrodes with a model approach focusing on optimally transferring speech information, has significant potential for improving outcome. This is an interesting line of research which requires further exploration in our target group, although the time-consuming measurements might be a practical obstacle to find sufficient numbers of subjects willing to participate.

\section{Measuring outcome: a different perspective}

In chapters 2 and 3 we focused on how technical aspects of $\mathrm{Cl}$ processing are related to speech understanding outcomes in the group of early-deafened $\mathrm{Cl}$ users. In chapter 6 however, one of the aims was to explore how we should measure outcome in our target group in the first place. Traditional outcome measures for $\mathrm{Cl}$ users mainly include open-set word and sentence recognition tests in quiet, evolving towards measures in noise as performance of the average, postlingual $\mathrm{Cl}$ user improves, as illustrated by the recommendations of the New Minimum Speech Test Battery (MSTB) for adult cochlear implant users (Auditory Potential, 2011). This shift towards more complex outcome measures tends to forget that a significant proportion of adult $\mathrm{Cl}$ users, especially in the early-deafened group, still struggles to obtain any open-set speech recognition benefit at all (Caposecco et al., 2012; Heywood et al., 2016; O'Gara et al., 2016; Rousset et al., 2016). In chapter 6 our subject group of 27 early-deafened subjects obtained a mean 1 year postoperative CVC word recognition score of $44.2 \%$, which corresponds with the range of outcomes (20-49\%) of the studies reviewed in chapter 4 . Out of the 27 subjects in our subject group, however, 7 obtained no more than $10 \%$ and 10 subjects scored less than $30 \%$ on this open-set CVC word test. This latter group of 10, the so-called "poor performers", did show significant benefit on a closed-set measure of suprasegmental cues as well as on a measure of speech tracking, assessing the general ease of communication. Part 1 of our systematic review (chapter 4) equally found measures of closed-set speech understanding to be valuable and although there were no studies included that assessed speech tracking, there is a similarity with tests assessing auditory-visual communication. The latter tests showed significant benefits in almost all studies, pointing to the capability of early-deafened subjects to combine new, auditory information from the $\mathrm{Cl}$ with visual information obtained through lip reading, thus obtaining a higher level of speech understanding. Our recommendation therefore is to substitute traditional outcome measures of speech understanding with closed-set measures and measures assessing the 
combination of auditory and visual information in this patient population, especially in poor performers. To this end, we are currently working on an extended validation of the (Dutch) texts used for the speech tracking test we implemented in this population, in order to improve their clinical usefulness.

In addition, it was shown in chapter 6 that the early-deafened subject group, but also the subgroup of poor performers, showed significant benefit on the questionnaire on subjective benefit, the latter despite the absence of a significant improvement on openset speech understanding. Very positive outcomes for hearing-related quality of life were confirmed in chapter 4 . In general, changes in subjective experience after cochlear implantation were most clear for domains related to sound perception, sense of safety and communication, but were less obvious with respect to social life or self-esteem, which we believe might be due to the long-standing nature of the subjects' hearing impairment. The questionnaire used in chapter 6 , which was specifically developed to be used in our target population, fulfils the need for a questionnaire that takes the particularities of this patient group into account. On the other hand, the limited number of subjects it was submitted to unfortunately did not allow for an extensive validation. This is an issue that should be further addressed prior to a wider use of the questionnaire. Finally, the lack of a strong relation between subjective benefit on one hand and speech perception outcome on the other, emphasizes that a subjective measure of outcome is indispensable in our target population to obtain a truthful assessment of outcome.

\section{Predicting performance}

The second part of the systematic review presented in chapter 5 identified three variables as having good potential in predicting (speech understanding) outcomes in the earlydeafened population; one of those, the preoperative word recognition score, was one of two remaining significant predictors in the multiple regression analysis performed on our own study group as well (chapter 6). The two other variables that were identified as good predictors in chapter 5 , i.e. preoperative speech intelligibility and communication mode in childhood, were not incorporated in our own analysis. This can mainly be attributed to a lack of data on these variables as they are not systematically assessed in our $\mathrm{Cl}$ candidates. Inversely the second significant predictor of our own regression analysis, preoperative hearing thresholds of the ear to be implanted, was not clearly related to outcomes in the studies of the review; the thresholds of the better ear showed more predictive potential. The small number of subjects our multiple regression analysis was based on inevitably limits the generalizability of the observed outcomes. There is clearly a need for research in larger groups of early-deafened subjects, which at the same presents a major challenge given the relatively small proportions of early-deafened $\mathrm{Cl}$ users implanted in most clinics. A multicentre study, as has been performed in postlingually 
deafened $\mathrm{Cl}$ users (Blamey et al., 2013), could potentially provide an answer to this problem.

Apart from sample size issues, the major limitations observed in chapter 5 which should be taken into account by future studies, were the lack of clear definitions of the included variables, as well as inappropriate statistical analysis. Attention also needs to be paid to the choice of predictors to include. Until now, the focus of most studies - including our own - has been on demographic factors concerning hearing history and the implantation itself. The combination of these factors has never been be able to explain much more than about $60 \%$ of the variation in speech understanding outcome in this population, with a great deal of variables mainly explaining the same variation (chapter 6, Caposecco et al., 2012; Kraaijenga et al., 2016; O'Gara et al., 2016; van Dijkhuizen et al., 2011).

Recently, Pisoni et al. (2017) suggested that the missing predictors should be sought in measures of underlying cognitive processing, at least in postlingually deaf adults. In that respect, Kral et al. (2016) suggest that the limited auditory experience during the development of early-deafened subjects not only disturbs the perception of spoken language and development of the auditory system itself, but also affects the development of neurocognitive functions such as concept formation and executive functioning, and results in altered connections between the auditory system and other brain systems. Individual variability in the development of neural circuits in response to auditory deprivation is thought to contribute to the observed variability in outcomes with a cochlear implant later on. Subjects, who are for instance better at integrating top-down information streams with the incoming auditory cues, are more likely to become better performers. These ideas can be extended to the population of late-implanted but earlydeafened $\mathrm{Cl}$ users: the extent to which these higher-order neurocognitive functions have been developed might be determined by the amount of auditory input in early childhood (which is related to the exact age at onset of deafness, the amount of residual hearing and the adequacy of early hearing aid rehabilitation), along with the aforementioned individual variability. It would thus be an interesting area for further investigations to assess if and which measures of neurocognitive functioning could be used within our target population, and whether they are valuable as predictors in multiple regression analyses.

Finally, when taking in mind that a measure for auditory-visual speech recognition is often a more relevant way to assess outcome in early-deafened $\mathrm{Cl}$ users (chapter 4 and 6), it would equally be interesting for future studies in our target population to find relevant predictors for such an auditory-visual outcome measure, instead of just focusing on auditory-only benefits. As the ability to integrate these communication modes potentially requires different skills, the relevant predictors might differ as well. 


\section{Reflecting on implantation criteria}

Although cochlear implantation in patients with early-onset deafness remains controversial within the Deaf community, especially when it concerns young children (Sparrow, 2010), it was not within the scope of this thesis to go further into this debate. With respect to early-deafened adults we believe that it is a personal choice for every individual to sign up, or not, for cochlear implantation. As the technique of cochlear implantation was not yet applied on a large scale in the Netherlands until the late 90's, parents of children born deaf or severely hearing impaired before that time did not have the possibility to choose for a cochlear implant, as is the case nowadays.

The results presented in chapters 4, 5 and 6 urge us to reflect on the implantation criteria for those early-deafened adults who are interested in increasing their access to the hearing world and are now candidates for cochlear implantation. A very interesting observation was that the evidence so far does not strongly support the notion that subjects showing large changes in hearing-related quality of life are also the ones obtaining significant speech understanding improvements. Although more research is definitely required, a strong correlation between auditory gains and subjective benefits could be found neither in our study group nor in the review. In addition, the anticipated gain in auditory-only speech understanding with $\mathrm{Cl}$ is generally limited, whereas it was also demonstrated that when subjects are evaluated with assessment methods targeted to their level of auditory functioning, benefits often do arise, but they might be on domains other than open-set speech understanding.

Based on these observations, we suggest that implantation criteria in this population should not only look at the expected level of auditory-only speech understanding, but also include alternative domains of potential benefit including auditory-visual speech recognition and hearing-related quality of life. 

Summary 

Cochlear implantation has become a well-established treatment option in postlingually deafened patients, with most of them obtaining good results on traditional tests for speech recognition. When early-deafened patients receive a cochlear implant in (late) adolescence or adulthood, however, speech recognition results are often not so favorable. The added value of a $\mathrm{Cl}$ in this patient group therefore remains unclear and subject to discussion. This is reflected by the doubts of many $\mathrm{Cl}$ clinics whether or not to implant early-deafened, adult or adolescent $\mathrm{Cl}$-candidates. More knowledge of both the expected outcomes with a $\mathrm{Cl}$ within this group, and the factors that might influence them, is therefore of great importance for candidacy decisions. This thesis addressed a number of important issues related to cochlear implantation in this very specific subject group, from factors relevant in determining pre-implant candidacy (chapters 4, 5 and 6), over aspects related to $\mathrm{Cl}$ signal processing and fitting (chapters $2 \& 3$ ), to optimization of outcome assessment (chapter 6). The ultimate goal was to gain more insight into the field of cochlear implantation in the early-deafened but late-implanted patient population, in order to improve patient selection and (the assessment of) cochlear implant outcome.

The general introduction in chapter 1 starts with a brief description of the mechanism of a cochlear implant, explaining the relation with spectral and temporal processing. It continues with an overview of how auditory deprivation in early childhood affects the development of the auditory system. It then describes a number of issues and problems related to cochlear implantation in this population, and the corresponding research questions that emerged out of these, which we aimed to answer in the different chapters of this thesis.

Chapter 2 evaluates the extent to which both early-deafened and postlingually deafened adult $\mathrm{Cl}$ users are able to process temporal envelope cues. Since these cues are present also in the $\mathrm{Cl}$ signal, it was equally of interest to us whether the ability to process them is related to speech recognition outcomes. We reported worse amplitude modulation detection tresholds (AMDTs) - a measure of temporal processing - for the early-deafened subjects compared to a postlingually deafened group. None of the early-deafened subjects were able to detect very fast $(>100 \mathrm{~Hz})$ modulations. In addition, better speech recognition scores were obtained in subjects having better amplitude modulation thresholds that degraded less quickly with increasing modulation frequency. Another difference between both subject groups was that significant correlations between the detection of slow modulations and speech recognition were present only for the earlydeafened but not for the postlingual group, the reason for which currently remains unclear.

In chapter 3, the spectral processing abilities of a small group of early-deafened $\mathrm{Cl}$ users were investigated by means of an adaptive electrode discrimination testing procedure. 
Results showed that subjects are not able to perceptually discriminate between all adjacent electrodes, especially when electrodes are located in the basal region of the cochlea. The obtained outcomes were subsequently used to create an adapted cochlear implant fitting containing only discriminable electrodes and subjects were given four weeks to adjust to this new fitting. Results revealed no significant differences between the subjects' basic fitting and the new, experimental fitting, with respect to speech recognition performance (in quiet or noise), listening effort or spectral ripple discrimination. Subjective appreciation was generally worse for the experimental fitting. A number of factors related to the creation of the experimental map, more specifically the reduction of the number of maxima, the adaption period of (only) 4 weeks and the mainly basal location of the deactivated electrodes, which is a region less relevant for speech understanding, might explain the lack of an improvement with the experimental map.

Chapters 4 and 5 present a systematic review on cochlear implantation in early-deafened, late-implanted adolescent and adult $\mathrm{Cl}$ users. In chapter 4 we focus on postoperative outcomes and in chapter 5 on factors that are predictive of these outcomes. The systematic search yielded 1449 unique articles. Further selection identified 38 studies meeting the eligibility criteria for part 1 , and 13 studies that were prognostic in nature and thus relevant for part 2 . In chapter 4 we reported significant improvements for open-set speech recognition in the majority of the included studies, and observed significant improvements for measures assessing closed-set and auditory-visual speech understanding as well as hearing-related quality of life in almost all studies involved. In chapter 5 , the synthesis of a vast variety of potential prognostic factors, assessed in the included studies, revealed three factors with good predictive potential: communication mode (mainly in childhood), preoperative speech intelligibility, and preoperative speech recognition scores. Furthermore, suggestions were made for a number of other factors that are worth further investigation.

Quality assessment of the studies included in the review also revealed several methodological weaknesses and sources of bias which might have impacted the observed outcomes, albeit to an unknown extent. Main issues included inadequate statistical testing, often related to the limited sample size, lack of information on relevant study aspects, such as measurement methods of prognostic factors, and variation between the included study groups due to unspecified implantation- and study inclusion criteria.

The prospective study presented in chapter 6, which included 27 early-deafened adults that received a cochlear implant, demonstrated significant improvements in auditory performance in accordance with the results of the review, as well as self-perceived benefits on a questionnaire that was specifically developed for this population of $\mathrm{Cl}$ users. An interesting result was that a subgroup of poor performers, i.e. subjects failing to show an improvement on open-set speech recognition tests, did obtain signifcant benefit when 
assessed with measures targeted to their level of performance (such as closed-set tests and speech tracking). A correlation between objective and subjective outcome measures could not be found. This emphasizes the need for a subjective evaluation of outcomes also in this patient group, as measures of speech understanding do not seem to fully capture the indvidually experienced benefit after cochlear implantation. In the last study objective of chapter 6, two factors were identified that were able to explain $63.5 \%$ of the variation in outcomes of the included subjects, i.e. preoperative PTA and preoperative CNC word recognition (both of the implanted ear). Where the latter was found to be relevant in chapter 5 as well, the first was not, demonstrating the need for further research especially in larger subject groups.

In the general discussion (chapter 7) the main findings of this thesis are discussed in light of their implications for the clinical field, and suggestions for further research are presented. A number of difficulties concerning the definition of our patient group of interest, are discussed. Suggestions are made as to how fitting parameters can be further explored and, when integrated with for instance mathematical modeling, potentially improve outcomes. In order to better predict performance with $\mathrm{Cl}$ in our patient group, research in larger study groups is needed and new prognostic factors - such as those revealing underlying cognitive processes - should be investigated. We conclude that a cochlear implant in early-deafened, late-implanted adults and adolescents generally has a positive outcome. However, less traditional outcome measures, which focus on the patients' individual performance level and take subjective benefit into account, are required to reveal the true impact of a cochlear implant for these patients. 

Nederlandse samenvatting 

In dit proefschrift staat de patiënt centraal die vanaf geboorte doof of op zeer jonge leeftijd doof geworden is, én pas (laat) in de adolescentie of op volwassen leeftijd een cochleair implantaat $(\mathrm{Cl})$ heeft gekregen. Bij cochleaire implantatie wordt een kleine elektrode-drager chirurgisch ingebracht in het slakkenhuis van de patiënt. Wanneer dit inwendige implantaat verbinding maakt met een uitwendige geluidsprocessor, zorgt dit systeem ervoor dat akoestische signalen omgezet worden in een reeks elektrische pulsen. Deze elektrische pulsen stimuleren de auditieve zenuwuiteinden in het slakkenhuis, die ervoor zorgen dat het signaal verder geleid wordt tot in de hersenen en zo uiteindelijk "gehoord" wordt door de patiënt. Bij postlinguaal dove volwassenen - dit zijn patiënten waarbij het gehoorverlies is ontstaan ná het einde van de spraak-taalontwikkeling - is cochleaire implantatie een veel voorkomende behandelingsmethode geworden. Het merendeel van deze patiënten behaalt dan ook goede tot zeer goede resultaten op traditionele tests voor spraakverstaan. Dit staat in contrast met de resultaten die worden behaald door vele vroeg-dove volwassenen. De meerwaarde van een $\mathrm{Cl}$ bij deze patiëntengroep staat dan ook nog regelmatig ter discussie. Dit zien we ook terug in de twijfels die veel $\mathrm{Cl}$ centra hebben over het al dan niet implanteren van vroeg-dove, volwassen $\mathrm{Cl}$-kandidaten. Meer kennis over zowel de te verwachten resultaten met een $\mathrm{Cl}$ bij deze patiëntengroep, als van de factoren die deze resultaten mee bepalen, is daarom van groot belang bij de patiëntenselectie.

In dit proefschrift wordt ingegaan op een aantal thema's die allemaal gerelateerd zijn aan cochleaire implantatie bij vroeg-dove volwassenen. Zo wordt onderzocht welke factoren relevant kunnen zijn bij het preoperatief bepalen van wie een geschikte $\mathrm{Cl}$-kandidaat is (hoofdstukken $5 \& 6$ ), hoe de verwerking van het $\mathrm{Cl}$-signaal en het fitten van de geluidsprocessor geoptimaliseerd kunnen worden (hoofdstukken 2 \& 3), en welke uitkomstmaten het meest geschikt zijn om de uiteindelijke prestaties met een $\mathrm{Cl}$ te evalueren (hoofdstukken $4 \& 6$ ). Het uiteindelijke doel van het proefschrift is om de bestaande kennis op het gebied van cochleaire implantatie bij vroeg-dove, maar laat geïmplanteerde patiënten te vergroten, en hiermee de patiëntenselectie en (het in kaart brengen van) de prestaties met een $\mathrm{Cl}$ te verbeteren.

De inleiding in hoofdstuk 1 start met een korte beschrijving van het werkingsmechanisme van een cochleair implantaat. Ten behoeve van de hoofdstukken 2 en 3 wordt ingegaan op begrippen als spectrale en temporele verwerking. Vervolgens wordt uitgelegd hoe auditieve deprivatie op zeer jonge leeftijd de ontwikkeling van het hele auditieve systeem beïnvloedt. Hierbij worden ook een aantal problemen en nieuwe ideeën besproken met betrekking tot cochleaire implantatie bij deze patiëntengroep. Tenslotte worden de hieruit voortvloeiende onderzoeksvragen, die we in de verschillende hoofdstukken van dit proefschrift trachten te beantwoorden, gepresenteerd. 
Hoofdstuk 2 brengt in kaart in welke mate zowel vroeg-dove als postlinguaal dove volwassen $\mathrm{Cl}$-gebruikers in staat zijn om temporele eigenschappen van een geluid te verwerken. Omdat er ook temporele aspecten aanwezig zijn in de omhullende van een $\mathrm{Cl}$ signaal, was een belangrijke onderzoeksvraag of de mate waarin een Cl-patiënt deze aspecten kan verwerken gerelateerd is aan zijn of haar resultaten op het gebied van spraakverstaan. De mate van temporele verwerking werd gemeten met behulp van een test voor amplitude-modulatiedetectie. Onze resultaten lieten zien dat de gevonden drempels op deze test minder goed waren voor vroeg-dove patiënten dan voor postlinguaal dove patiënten. Bij de groep vroeg-dove patiënten was tevens niemand in staat om heel snelle modulaties $(>100 \mathrm{~Hz})$ te detecteren. Zowel binnen de vroeg-dove als binnen de postlinguaal dove patiëntengroep werd een significante correlatie gevonden tussen de resultaten op de amplitudemodulatiedetectietest en de spraakverstaanscores met het $\mathrm{Cl}$. Een opvallend verschil was dat signficante correlaties tussen spraakverstaanscores en het detecteren van langzame modulaties enkel binnen de vroegdove groep gevonden werden. Waarom dit niet het geval was binnen de postlinguaal dove groep is onduidelijk.

In hoofdstuk 3 worden de spectrale verwerkingsmogelijkheden van een kleine groep vroeg-dove $\mathrm{Cl}$-dragers onderzocht aan de hand van een adaptieve testprocedure voor elektrodediscriminatie. De resultaten laten zien dat de onderzoekspatiënten niet in staat waren om alle naast elkaar liggende elektroden van elkaar te onderscheiden. Dit was met name het geval bij de elektroden die zich in het basale deel van de cochlea bevinden. De uitkomsten van de testprocedure werden vervolgens gebruikt om een aangepaste instelling van het $\mathrm{Cl}$ te creëren, die enkel discrimineerbare elektroden bevat. De proefpersonen kregen 4 weken de tijd om aan deze aangepaste instelling te wennen. Bij metingen na deze gewenningsperiode werden er geen significante verschillen gevonden wat betreft spraakverstaan (in stilte of in ruis), luisterinspanning, of discriminatie van spectrale "ripples" (een test voor spectrale resolutie) tussen de standaard instelling en de nieuwe, experimentele instelling. De subjectieve appreciatie was in het algemeen in het nadeel van de experimentele instelling. Mogelijke verklaringen voor het uitblijven van een verbetering met de experimentele $\mathrm{Cl}$-instelling zijn een te korte gewenningsperiode, de reductie van het aantal maxima bij de experimentele instelling en het feit dat het merendeel van de uitgeschakelde elektroden zich in het basale deel van de cochlea bevonden, hetgeen een minder relevante regio is voor spraakverstaan.

Hoofdstukken 4 en 5 presenteren ieder een deel van een systematische review bij vroegdove, laat-geïmplanteerde adolescente of volwassen $\mathrm{Cl}$-dragers. In het eerste deel (hoofdstuk 4) ligt de focus op de postoperatieve resultaten; in het tweede deel (hoofdstuk 5) staan de factoren die een potentieel voorspellende waarde hebben voor deze resultaten centraal. Het systematisch zoeken in de literatuur leverde 1449 unieke artikels 
op. De daarop volgende selectie identificeerde 38 studies die voldeden aan de vooropgestelde inclusiecriteria voor deel 1 , en 13 studies met een prognostische onderzoeksopzet die relevant waren voor deel 2 . In hoofdstuk 4 rapporteren we significante verbeteringen voor het open set spraakverstaan bij de meerderheid van de geïncludeerde studies. Patiënten scoorden ook significant hoger op testen voor gesloten set en auditief-visueel spraakverstaan na implantatie, alsook voor de gehoorgerelateerde kwaliteit van leven. In hoofdstuk 5 wordt een grote verscheidenheid aan potentieel voorspellende factoren besproken. Er worden drie factoren geïdentificeerd met goed voorspellend potentieel, namelijk: de wijze van communicatie (met name in de kindertijd), de preoperatieve spraakverstaanscores en de preoperatieve verstaanbaarheid van de eigen spraak van de patiënt. Verder worden er suggesties gedaan met betrekking tot welke andere factoren verder onderzocht dienen te worden in toekomstige studies.

Een evaluatie van de kwaliteit van de geïncludeerde studies liet diverse methodologische zwakheden en mogelijke bronnen van bias zien, die mogelijk invloed hebben gehad op de geobserveerde resultaten. De belangrijkste problemen hadden te maken met inadequate statistische testmethodes (vaak gerelateerd aan de beperkte grootte van de onderzoeksgroep), het ontbreken van relevante studie-informatie (zoals bij de meetmethode van voorspellende factoren) en variatie tussen de geïncludeerde studiegroepen van de diverse studies ten gevolge van niet-gespecificeerde implantatie- en inclusiecriteria.

De prospectieve studie bij 27 vroeg-dove $\mathrm{Cl}$-patiënten die gepresenteerd wordt in hoofdstuk 6 laat - net zoals de review - significante postoperatieve verbeteringen zien qua auditieve prestaties. Een significante verbetering werd ook gevonden wat betreft subjectief ervaren voordelen, gemeten met een vragenlijst die specifiek ontwikkeld werd voor deze populatie van $\mathrm{Cl}$-gebruikers. Een interessante bevinding was dat een deelgroep van slechte presteerders, d.w.z. een groep van patiënten die geen verbetering liet zien qua open set spraakverstaan, toch significant vooruitging wanneer zij onderzocht werd met testen die beter aangepast waren aan hun niveau van auditief functioneren (zoals gesloten set tests en "speech tracking"). Verder kon er geen correlatie gevonden worden tussen objectieve en subjectieve uitkomstmaten. Dit geeft aan dat het van belang is ook een subjectief evaluatie-instrument te gebruiken bij vroeg-dove $\mathrm{Cl}$-gebruikers, gezien de resultaten op de objectieve spraakverstaantesten los lijken te staan van de individueel ervaren voordelen. In het laatste deel van hoofdstuk 6 werden twee factoren geïdentificeerd die samen $63.5 \%$ van de variatie in uitkomsten van de geïncludeerde $\mathrm{Cl}$ gebruikers konden verklaren, namelijk de preoperatieve gehoordrempel en de preoperatieve CVC woordherkenningsscore (in beide gevallen van het geïmplanteerde oor). Waar de preoperatieve spraakverstaanscore ook in hoofdstuk 5 een significante voorspeller bleek te zijn, was de preoperative gehoordrempel dat niet. Dit toont aan dat er nood is aan verder onderzoek, met name in grotere patiëntengroepen. 
In de algemene discussie (hoofdstuk 7) worden de belangrijkste bevindingen van dit proefschrift besproken in het kader van hun mogelijke implicaties voor het klinische werkveld en worden er suggesties voor verder onderzoek gepresenteerd. Enkele moeilijkheden waarmee we geconfronteerd werden bij het definiëren van onze patiëntengroep worden hier besproken. Verder worden er suggesties gedaan hoe bepaalde parameters van de $\mathrm{Cl}$-instelling verder onderzocht kunnen worden en hoe deze, indien geïntegreerd met bepaalde wiskundige modellen, mogelijk tot verbeterde resultaten kunnen leiden. Om de prestaties met een $\mathrm{Cl}$ binnen onze patiëntengroep beter te kunnen voorspellen is met name meer onderzoek in grotere groepen nodig en dienen nieuwe voorspellers (zoals diegene die onderliggende cognitieve processen in kaart brengen) onderzocht te worden. Concluderend kunnen we stellen dat een cochleair implantaat bij vroeg-dove, laat-geïmplanteerde adolescenten en volwassenen doorgaans tot een positief resultaat leidt. Echter, de traditionele auditieve tests zijn voor deze groep veel minder geschikt. Het gebruik van aangepast testmateriaal, aangevuld met een instrument gericht op het in kaart brengen van subjectief ervaren voordelen, is van belang bij het bepalen van wat een $\mathrm{Cl}$ voor deze groep van patiënten betekent. 
References 

Anderson, E. S., Nelson, D. A., Kreft, H., Nelson, P. B., \& Oxenham, A. J. (2011). Comparing spatial tuning curves, spectral ripple resolution, and speech perception in cochlear implant users. J Acoust Soc Am, 130(1), 364-375.

Archbold, S., Lutman, M. E., \& Nikolopoulos, T. (1998). Categories of auditory performance: interuser reliability. Br J Audiol, 32(1), 7-12.

Arisi, E., Forti, S., Pagani, D., Todini, L., Torretta, S., Ambrosetti, U., \& Pignataro, L. (2010). Cochlear implantation in adolescents with prelinguistic deafness. Otolaryngol Head Neck Surg, 142(6), 804-808.

Arora, K., Vandali, A., Dowell, R., \& Dawson, P. (2011). Effects of stimulation rate on modulation detection and speech recognition by cochlear implant users. Int J Audiol, 50(2), 123-132.

Bacon, S. P., \& Viemeister, N. F. (1985). Temporal modulation transfer functions in normal-hearing and hearing-impaired listeners. Audiology, 24(2), 117-134.

Bassim, M. K., Buss, E., Clark, M. S., Kolln, K. A., Pillsbury, C. H., Pillsbury, H. C., 3rd, \& Buchman, C. A. (2005). MED-EL Combi40+ cochlear implantation in adults. Laryngoscope, 115(9), 15681573.

Bellelli, S., Forli, F., Berrettini, S., \& Turchetti, G. (2016). Cochlear implant in prelingually deafened oralist adults: Speech perception outcome, subjective benefits and quality of life improvement. Value in Health, 19 (7), A561.

Bench, J., Kowal, A., \& Bamford, J. (1979). The BKB (Bamford-Kowal-Bench) sentence lists for partially-hearing children. Br J Audiol, 13(3), 108-112.

Bernstein, L. E., Eberhardt, S. P., \& Auer, E. T., Jr. (2014). Audiovisual spoken word training can promote or impede auditory-only perceptual learning: prelingually deafened adults with late-acquired cochlear implants versus normal hearing adults. Front Psychol, 5, 934.

Blamey, P., Artieres, F., Baskent, D., Bergeron, F., Beynon, A., Burke, E., . . Lazard, D. S. (2013). Factors affecting auditory performance of postlinguistically deaf adults using cochlear implants: an update with 2251 patients. Audiol Neurootol, 18(1), 36-47.

Boons, K., \& Debruyne, J. (2011). Evaluatie van objectieve testmethodes voor prelinguaal dove volwassen $\mathrm{Cl}$-gebruikers. . Logopedie - Informatiemedium van de Vlaamse Vereniging voor Logopedisten, 24, 48-52.

Boothroyd, A., Hanin, L., \& Hnath, T. (1985). A sentence test of speech perception: reliability, set equivalence, and short term learning. Retrieved from CUNY Academic Works:

Bosco, E., Nicastri, M., Ballantyne, D., Viccaro, M., Ruoppolo, G., lonescu Maddalena, A., \& Mancini, P. (2013). Long term results in late implanted adolescent and adult $\mathrm{Cl}$ recipients. Eur Arch Otorhinolaryngol, 270(10), 2611-2620.

Bosman, A. J., \& Smoorenburg, G. F. (1995). Intelligibility of Dutch CVC syllables and sentences for listeners with normal hearing and with three types of hearing impairment. Audiology, 34(5), 260-284.

Brochier, T., McDermott, H. J., \& McKay, C. M. (2017). The effect of presentation level and stimulation rate on speech perception and modulation detection for cochlear implant users. J Acoust Soc Am, 141(6), 4097.

Buckley, K. A., \& Tobey, E. A. (2011). Cross-modal plasticity and speech perception in pre- and postlingually deaf cochlear implant users. Ear Hear, 32(1), 2-15.

Busby, P. A., \& Clark, G. M. (1996). Electrode discrimination by early-deafened cochlear implant patients. Audiology, 35(1), 8-22.

Busby, P. A., \& Clark, G. M. (2000). Electrode discrimination by early-deafened subjects using the cochlear limited multiple-electrode cochlear implant. Ear Hear, 21(4), 291-304.

Busby, P. A., Tong, Y. C., \& Clark, G. M. (1993). The perception of temporal modulations by cochlear implant patients. J Acoust Soc Am, 94(1), 124-131.

Butler, B. E., \& Lomber, S. G. (2013). Functional and structural changes throughout the auditory system following congenital and early-onset deafness: implications for hearing restoration. Front Syst Neurosci, 7, 92. 
Campbell, R., MacSweeney, M., \& Woll, B. (2014). Cochlear implantation (CI) for prelingual deafness: the relevance of studies of brain organization and the role of first language acquisition in considering outcome success. Front Hum Neurosci, 8, 834.

Caposecco, A., Hickson, L., \& Pedley, K. (2012). Cochlear implant outcomes in adults and adolescents with early-onset hearing loss. Ear Hear, 33(2), 209-220.

Cazals, Y., Pelizzone, M., Saudan, O., \& Boex, C. (1994). Low-pass filtering in amplitude modulation detection associated with vowel and consonant identification in subjects with cochlear implants. J Acoust Soc Am, 96(4), 2048-2054.

Chatterjee, M., \& Oberzut, C. (2011). Detection and rate discrimination of amplitude modulation in electrical hearing. J Acoust Soc Am, 130(3), 1567-1580.

Chatterjee, M., \& Yu, J. (2010). A relation between electrode discrimination and amplitude modulation detection by cochlear implant listeners. J Acoust Soc Am, 127(1), 415-426.

Chee, G. H., Goldring, J. E., Shipp, D. B., Ng, A. H., Chen, J. M., \& Nedzelski, J. M. (2004). Benefits of cochlear implantation in early-deafened adults: the Toronto experience. J Otolaryngol, 33(1), 26-31.

Cox, R. M., \& Alexander, G. C. (2002). The International Outcome Inventory for Hearing Aids (IOIHA): psychometric properties of the English version. Int J Audiol, 41(1), 30-35.

Craddock, L., Cooper, H., Riley, A., \& Wright, T. (2016). Cochlear implants for pre-lingually profoundly deaf adults. Cochlear Implants Int, 17 Suppl 1, 26-30.

Croghan, N. B. H., Krishnamoorthi, H., \& Smith, Z. M. (2013). What does spectral ripple discrimination at high ripple densities mean in cochlear implant listening experiments? Paper presented at the Conference on Implantable Auditory Prostheses, Lake Tahoe, California.

Cusumano, C., Friedmann, D. R., Fang, Y., Wang, B., Roland, J. T., Jr., \& Waltzman, S. B. (2017). Performance Plateau in Prelingually and Postlingually Deafened Adult Cochlear Implant Recipients. Otol Neurotol, 38(3), 334-338.

Dai, H., \& Micheyl, C. (2010). On the choice of adequate randomization ranges for limiting the use of unwanted cues in same-different, dual-pair, and oddity tasks. Atten Percept Psychophys, 72(2), 538-547.

Daneshi, A., \& Hassanzadeh, S. (2007). Cochlear implantation in prelingually deaf persons with additional disability. J Laryngol Otol, 121(7), 635-638.

Dau, T., Kollmeier, B., \& Kohlrausch, A. (1997). Modeling auditory processing of amplitude modulation. I. Detection and masking with narrow-band carriers. J Acoust Soc Am, 102(5 Pt 1), 2892-2905.

Davis, H., \& Silverman, S. R. (1978). Hearing and Deafness (4th ed.). London: Holt, Rinehart and Winston.

De Filippo, C. L., \& Scott, B. L. (1978). A method for training and evaluating the reception of ongoing speech. J Acoust Soc Am, 63(4), 1186-1192.

Derinsu, U., Ciprut, A., \& Akdas, F. (2002). Cochlear implant results in prelingual adults. Marmara Medical Journal, 15(3), 175-179.

Dorman, M. F., \& Spahr, A. J. (2006). Speech Perception by Adults with Multichannel Cochlear Implants. In Cochlear Implants: Thieme Medical Publishers, Inc.

Drennan, W. R., Anderson, E. S., Won, J. H., \& Rubinstein, J. T. (2014). Validation of a clinical assessment of spectral-ripple resolution for cochlear implant users. Ear Hear, 35(3), e9298.

Duchesne, L., Millette, I., Bherer, M., \& Gobeil, S. (2017). Auditory performance and subjective benefits in adults with congenital or prelinguistic deafness who receive cochlear implants during adulthood. Cochlear Implants Int, 18(3), 143-152.

Ebrahimi-Madiseh, A., Eikelboom, R. H., Jayakody, D. M., \& Atlas, M. D. (2016). Speech perception scores in cochlear implant recipients: An analysis of ceiling effects in the CUNY sentence 
test (Quiet) in post-lingually deafened cochlear implant recipients. Cochlear Implants Int, 17(2), 75-80.

Eisenberg, L. S. (1982). Use of the cochlear implant by the prelingually deaf. Ann Otol Rhinol Laryngol Suppl, 91(2 Pt 3), 62-66.

Erber, N. P., \& Alencewicz, C. M. (1976). Audiologic evaluation of deaf children. J Speech Hear Disord, 41(2), 256-267.

Etymōtic Research, I. (2005). BKB-SIN test. Speech-in-Noise Test. Retrieved from: www.etymotic.com

Fayad, J. N., \& Linthicum, F. H., Jr. (2006). Multichannel cochlear implants: relation of histopathology to performance. Laryngoscope, 116(8), 1310-1320.

Feeny, D., Furlong, W., Saigal, S., \& Sun, J. (2004). Comparing directly measured standard gamble scores to HUI2 and HUI3 utility scores: group- and individual-level comparisons. Soc Sci Med, 58(4), 799-809.

Feeny, D., Furlong, W., Torrance, G. W., Goldsmith, C. H., Zhu, Z., DePauw, S., . . Boyle, M. (2002). Multiattribute and single-attribute utility functions for the health utilities index mark 3 system. Med Care, 40(2), 113-128.

Francart, T., van Wieringen, A., \& Wouters, J. (2008). APEX 3: a multi-purpose test platform for auditory psychophysical experiments. J Neurosci Methods, 172(2), 283-293.

Fraser, M., \& McKay, C. M. (2012). Temporal modulation transfer functions in cochlear implantees using a method that limits overall loudness cues. Hear Res, 283(1-2), 59-69.

Friesen, L. M., Shannon, R. V., \& Cruz, R. J. (2005). Effects of stimulation rate on speech recognition with cochlear implants. Audiol Neurootol, 10(3), 169-184.

Fu, Q. J. (2002). Temporal processing and speech recognition in cochlear implant users. Neuroreport, 13(13), 1635-1639.

Fu, Q. J., Shannon, R. V., \& Galvin, J. J., 3rd. (2002). Perceptual learning following changes in the frequency-to-electrode assignment with the Nucleus-22 cochlear implant. J Acoust Soc Am, 112(4), 1664-1674.

Galvin, J. J., 3rd, \& Fu, Q. J. (2005). Effects of stimulation rate, mode and level on modulation detection by cochlear implant users. J Assoc Res Otolaryngol, 6(3), 269-279.

Galvin, J. J., 3rd, \& Fu, Q. J. (2009). Influence of stimulation rate and loudness growth on modulation detection and intensity discrimination in cochlear implant users. Hear Res, 250(1-2), 46-54.

Galvin, J. J., 3rd, Fu, Q. J., Oba, S., \& Baskent, D. (2014). A method to dynamically control unwanted loudness cues when measuring amplitude modulation detection in cochlear implant users. J Neurosci Methods, 222, 207-212.

Garadat, S. N., Zwolan, T. A., \& Pfingst, B. E. (2012). Across-site patterns of modulation detection: relation to speech recognition. J Acoust Soc Am, 131(5), 4030-4041.

Gifford, R. H., Noble, J. H., Camarata, S. M., Sunderhaus, L. W., Dwyer, R. T., Dawant, B. M., . . . Labadie, R. F. (2018). The Relationship Between Spectral Modulation Detection and Speech Recognition: Adult Versus Pediatric Cochlear Implant Recipients. Trends Hear, 22, 2331216518771176.

Gifford, R. H., Shallop, J. K., \& Peterson, A. M. (2008). Speech recognition materials and ceiling effects: considerations for cochlear implant programs. Audiol Neurootol, 13(3), 193-205.

Gnansia, D., Lazard, D. S., Leger, A. C., Fugain, C., Lancelin, D., Meyer, B., \& Lorenzi, C. (2014). Role of slow temporal modulations in speech identification for cochlear implant users. Int J Audiol, 53(1), 48-54.

Gordon, K. A., Wong, D. D., Valero, J., Jewell, S. F., Yoo, P., \& Papsin, B. C. (2011). Use it or lose it? Lessons learned from the developing brains of children who are deaf and use cochlear implants to hear. Brain Topogr, 24(3-4), 204-219.

Guo, B., Moga, C., Harstall, C., \& Schopflocher, D. (2016). A principal component analysis is conducted for a case series quality appraisal checklist. J Clin Epidemiol, 69, 199-207 e192. 
Hacker, M. J., \& Ratcliff, R. (1979). A revised table of d' for M-alternative forced choice. Perception \& Psychophysics, 26(2), 168-170.

Hamilton, D. F., Lane, J. V., Gaston, P., Patton, J. T., Macdonald, D., Simpson, A. H., \& Howie, C. R. (2013). What determines patient satisfaction with surgery? A prospective cohort study of 4709 patients following total joint replacement. BMJ Open, 3(4).

Hardie, N. A., \& Shepherd, R. K. (1999). Sensorineural hearing loss during development: morphological and physiological response of the cochlea and auditory brainstem. Hear Res, 128(1-2), 147-165.

Hayden, J. A., Cote, P., \& Bombardier, C. (2006). Evaluation of the quality of prognosis studies in systematic reviews. Ann Intern Med, 144(6), 427-437.

Hayden, J. A., van der Windt, D. A., Cartwright, J. L., Cote, P., \& Bombardier, C. (2013). Assessing bias in studies of prognostic factors. Ann Intern Med, 158(4), 280-286.

Henry, B. A., McKay, C. M., McDermott, H. J., \& Clark, G. M. (2000). The relationship between speech perception and electrode discrimination in cochlear implantees. J Acoust Soc Am, 108(3 Pt 1), 1269-1280.

Henshall, K. R., \& McKay, C. M. (2001). Optimizing electrode and filter selection in cochlear implant speech processor maps. J Am Acad Audiol, 12(9), 478-489.

Heywood, R. L., Vickers, D. A., Pinto, F., Fereos, G., \& Shaida, A. (2016). Assessment and Outcome in Non-Traditional Cochlear Implant Candidates. Audiol Neurootol, 21(6), 383-390.

Hinderink, J. B., Krabbe, P. F., \& Van Den Broek, P. (2000). Development and application of a healthrelated quality-of-life instrument for adults with cochlear implants: the Nijmegen cochlear implant questionnaire. Otolaryngology-Head and Neck Surgery, 123(6), 756-765.

Hinderink, J. B., Mens, L. H., Brokx, J. P., \& van den Broek, P. (1995). Performance of prelingually and postlingually deaf patients using single-channel or multichannel cochlear implants. Laryngoscope, 105(6), 618-622.

Holden, L. K., Finley, C. C., Firszt, J. B., Holden, T. A., Brenner, C., Potts, L. G., . . Skinner, M. W. (2013). Factors affecting open-set word recognition in adults with cochlear implants. Ear Hear, 34(3), 342-360.

Holt, R. F., \& Kirk, K. I. (2005). Speech and language development in cognitively delayed children with cochlear implants. Ear Hear, 26(2), 132-148.

Johnson, K., \& Wiley, S. (2009). Cochlear implantation in children with multiple disabilities. In L. S. Eisenberg (Ed.), Clinical Management of Children with Cochlear Implants. San Diego, CA, USA: Plural Publishing Inc.

Jones, G. L., Won, J. H., Drennan, W. R., \& Rubinstein, J. T. (2013). Relationship between channel interaction and spectral-ripple discrimination in cochlear implant users. J Acoust Soc Am, 133(1), 425-433.

Kaandorp, M. W., Smits, C., Merkus, P., Festen, J. M., \& Goverts, S. T. (2017). Lexical-Access Ability and Cognitive Predictors of Speech Recognition in Noise in Adult Cochlear Implant Users. Trends Hear, 21, 2331216517743887.

Kaplan, D. M., Shipp, D. B., Chen, J. M., Ng, A. H., \& Nedzelski, J. M. (2003). Early-deafened adult cochlear implant users: assessment of outcomes. J Otolaryngol, 32(4), 245-249.

Klop, W. M., Boermans, P. P., Ferrier, M. B., van den Hout, W. B., Stiggelbout, A. M., \& Frijns, J. H. (2008). Clinical relevance of quality of life outcome in cochlear implantation in postlingually deafened adults. Otology and Neurotology, 29(5), 615-621.

Klop, W. M., Briaire, J. J., Stiggelbout, A. M., \& Frijns, J. H. (2007). Cochlear implant outcomes and quality of life in adults with prelingual deafness. Laryngoscope, 117(11), 1982-1987.

Koch, D. B., Osberger, M. J., Segel, P., \& Kessler, D. (2004). HiResolution and conventional sound processing in the HiResolution bionic ear: using appropriate outcome measures to assess speech recognition ability. Audiol Neurootol, 9(4), 214-223.

Kos, M. I., Deriaz, M., Guyot, J. P., \& Pelizzone, M. (2009). What can be expected from a late cochlear implantation? Int J Pediatr Otorhinolaryngol, 73(2), 189-193. 
Kraaijenga, V. J., Smit, A. L., Stegeman, I., Smilde, J. J., van Zanten, G. A., \& Grolman, W. (2016). Factors that influence outcomes in cochlear implantation in adults, based on patientrelated characteristics - a retrospective study. Clin Otolaryngol, 41(5), 585-592.

Kral, A. (2007). Unimodal and cross-modal plasticity in the 'deaf' auditory cortex. Int J Audiol, 46(9), 479-493.

Kral, A. (2013). Auditory critical periods: a review from system's perspective. Neuroscience, 247, 117133.

Kral, A., Kronenberger, W. G., Pisoni, D. B., \& O'Donoghue, G. M. (2016). Neurocognitive factors in sensory restoration of early deafness: a connectome model. Lancet Neurol, 15(6), 610-621.

Kral, A., \& Sharma, A. (2012). Developmental neuroplasticity after cochlear implantation. Trends Neurosci, 35(2), 111-122.

Kral, A., Tillein, J., Heid, S., Hartmann, R., \& Klinke, R. (2005). Postnatal cortical development in congenital auditory deprivation. Cereb Cortex, 15(5), 552-562.

Kral, A., Yusuf, P. A., \& Land, R. (2017). Higher-order auditory areas in congenital deafness: Topdown interactions and corticocortical decoupling. Hear Res, 343, 50-63.

Kumar, R. S., Mawman, D., Sankaran, D., Melling, C., O'Driscoll, M., Freeman, S. M., \& Lloyd, S. K. (2016). Cochlear implantation in early deafened, late implanted adults: Do they benefit? Cochlear Implants Int, 17 Suppl 1, 22-25.

Kwon, B. J., \& van den Honert, C. (2006). Dual-electrode pitch discrimination with sequential interleaved stimulation by cochlear implant users. J Acoust Soc Am, 120(1), EL1-6.

Laneau, J., \& Wouters, J. (2004). Multichannel place pitch sensitivity in cochlear implant recipients. J Assoc Res Otolaryngol, 5(3), 285-294.

Lazard, D. S., Giraud, A. L., Gnansia, D., Meyer, B., \& Sterkers, O. (2012). Understanding the deafened brain: implications for cochlear implant rehabilitation. Eur Ann Otorhinolaryngol Head Neck Dis, 129(2), 98-103.

Lazard, D. S., Vincent, C., Venail, F., Van de Heyning, P., Truy, E., Sterkers, O., . . Blamey, P. J. (2012). Pre-, per- and postoperative factors affecting performance of postlinguistically deaf adults using cochlear implants: a new conceptual model over time. PLoS One, 7(11), e48739.

Leek, M. R., Hanna, T. E., \& Marshall, L. (1992). Estimation of psychometric functions from adaptive tracking procedures. Percept Psychophys, 51(3), 247-256.

Lenarz, M., Sonmez, H., Joseph, G., Buchner, A., \& Lenarz, T. (2012). Long-term performance of cochlear implants in postlingually deafened adults. Otolaryngol Head Neck Surg, 147(1), 112-118.

Levitt, H. (1971). Transformed up-down methods in psychoacoustics. J Acoust Soc Am, 49(2), Suppl 2:467+.

Little, R. J. A., \& Rubin, D. B. (2002). Statistical Analysis with Missing Data (2nd ed.). New York: Wiley.

Loeffler, C., Aschendorff, A., Burger, T., Kroeger, S., Laszig, R., \& Arndt, S. (2010). Quality of Life Measurements after Cochlear Implantation. The Open Otorhinolaryngology Journal, 4(1), 47-54.

Luo, X., Fu, Q. J., Wei, C. G., \& Cao, K. L. (2008). Speech recognition and temporal amplitude modulation processing by Mandarin-speaking cochlear implant users. Ear Hear, 29(6), 957970.

Luo, X., Galvin, J. J., \& Fu, Q. J. (2010). Effects of stimulus duration on amplitude modulation processing with cochlear implants. J Acoust Soc Am, 127(2), EL23-29.

Mantokoudis, G., Vischer, M., Dubach, P., Kompis, M., Eberhard, E. S., \& Senn, P. (2011). Assessment of speech perception and communication skills in adolescents with cochlear implants for pre- and peri-lingual deafness. $B-E N T, 7(4), 251-259$.

Martini, A., Castiglione, A., Bovo, R., Vallesi, A., Gabelli, C. (2014). Aging, cognitive load, dementia and hearing loss. Audiol Neurootol, 19(suppl 1), 2-5. 
McDermott, H. J., McKay, C. M., \& Vandali, A. E. (1992). A new portable sound processor for the University of Melbourne/Nucleus Limited multielectrode cochlear implant. J Acoust Soc Am, 91(6), 3367-3371.

McKay, C. M. (2005). Spectral processing in cochlear implants. Int Rev Neurobiol, 70, 473-509.

McKay, C. M., \& Henshall, K. R. (2010). Amplitude modulation and loudness in cochlear implantees. J Assoc Res Otolaryngol, 11(1), 101-111.

Medina, M. D. M., Polo, R., Gutierrez, A., Muriel, A., Vaca, M., Perez, C., . . Cobeta, I. (2017). Cochlear Implantation in Postlingual Adult Patients With Long-Term Auditory Deprivation. Otol Neurotol, 38(8), e248-e252.

Millette, I., Gobeil, S., Bherer, M., \& Duchesne, L. (2011). Cochlear implants in adults with prelinguistic deafness: do auditory gains match the subjective benefits? Cochlear Implants Int, 12 Suppl 1, S78-81.

Moga, C., Guo, B., Schopflocher, D., \& Harstall, C. (2012). Development of a quality appraisal tool for case series studies using a modified Delphi technique. Edmonton $\mathrm{AB}$.

Moody-Antonio, S., Takayanagi, S., Masuda, A., Auer, E. T., Jr., Fisher, L., \& Bernstein, L. E. (2005). Improved speech perception in adult congenitally deafened cochlear implant recipients. Otol Neurotol, 26(4), 649-654.

Moon, I. J., Kim, E. Y., Jeong, J. O., Chung, W. H., Cho, Y. S., \& Hong, S. H. (2012). The influence of various factors on the performance of repetition tests in adults with cochlear implants. Eur Arch Otorhinolaryngol, 269(3), 739-745.

Most, T., Rothem, H., \& Luntz, M. (2009). Auditory, visual, and auditory-visual speech perception by individuals with cochlear implants versus individuals with hearing aids. Am Ann Deaf, 154(3), 284-292.

Most, T., Shrem, H., \& Duvdevani, I. (2010). Cochlear implantation in late-implanted adults with prelingual deafness. Am J Otolaryngol, 31(6), 418-423.

Mulder, J., Straatman, L., Huinck, W., Langereis, M., \& Snik, A. (2010). Cochlear implantation in lateimplanted prelingually deaf adults: Changes in Quality of life. Paper presented at the 11th International Conference on Cochlear Implants and Other Implantable Auditory Technologies, Stockholm Sweden.

Auditory Potential (2011). New Minimum Speech Test Battery (MSTB) for adult cochlear implant users. Retrieved from http://www.auditorypotential.com/MSTBfiles/MSTBManual201106-20\%20.pdf

Nikolopoulos, T. P., Archbold, S. M., Wever, C. C., \& Lloyd, H. (2008). Speech production in deaf implanted children with additional disabilities and comparison with age-equivalent implanted children without such disorders. Int J Pediatr Otorhinolaryngol, 72(12), 18231828.

Nilsson, M., Soli, S. D., \& Sullivan, J. A. (1994). Development of the Hearing in Noise Test for the measurement of speech reception thresholds in quiet and in noise. J Acoust Soc Am, 95(2), 1085-1099.

O'Gara, S. J., Cullington, H. E., Grasmeder, M. L., Adamou, M., \& Matthews, E. S. (2016). Factors Affecting Speech Perception Improvement Post Implantation in Congenitally Deaf Adults. Ear Hear, 37(6), 671-679.

Owens, E., \& Raggio, M. (1988). Performance inventory for profound and severe loss (PIPSL). J Speech Hear Disord, 53(1), 42-56.

Peasgood, A., Brookes, N., \& Graham, J. (2003). Performance and benefit as outcome measures following cochlear implantation in non-traditional adult candidates: a pilot study. Cochlear Implants Int, 4(4), 171-190.

Pfingst, B. E. (2011). Effects of electrode configuration on cochlear implant modulation detection thresholds. J Acoust Soc Am, 129(6), 3908-3915.

Pfingst, B. E., Burkholder-Juhasz, R. A., Zwolan, T. A., \& Xu, L. (2008). Psychophysical assessment of stimulation sites in auditory prosthesis electrode arrays. Hear Res, 242(1-2), 172-183. 
Pfingst, B. E., Xu, L., \& Thompson, C. S. (2007). Effects of carrier pulse rate and stimulation site on modulation detection by subjects with cochlear implants. J Acoust Soc Am, 121(4), 22362246.

Pisoni, D. B., Kronenberger, W. G., Harris, M. S., \& Moberly, A. C. (2017). Three challenges for future research on cochlear implants. World J Otorhinolaryngol Head Neck Surg, 3(4), 240-254.

Plant, K. L., Whitford, L. A., Psarros, C. E., \& Vandali, A. E. (2002). Parameter selection and programming recommendations for the ACE and CIS speech-processing strategies in the Nucleus 24 cochlear implant system. Cochlear Implants Int, 3(2), 104-125.

Qazi, O. U., van Dijk, B., Moonen, M., \& Wouters, J. (2013). Understanding the effect of noise on electrical stimulation sequences in cochlear implants and its impact on speech intelligibility. Hear Res, 299, 79-87.

Robinson, K., Gatehouse, S., \& Browning, G. G. (1996). Measuring patient benefit from otorhinolaryngological surgery and therapy. Ann Otol Rhinol Laryngol, 105(6), 415-422.

Roditi, R. E., Poissant, S. F., Bero, E. M., \& Lee, D. J. (2009). A predictive model of cochlear implant performance in postlingually deafened adults. Otol Neurotol, 30(4), 449-454.

Rosen, S. (1992). Temporal information in speech: acoustic, auditory and linguistic aspects. Philos Trans R Soc Lond B Biol Sci, 336(1278), 367-373.

Rousset, A., Dowell, R., \& Leigh, J. (2016). Receptive language as a predictor of cochlear implant outcome for prelingually deaf adults. Int J Audiol, 55 Suppl 2, S24-30.

Rubin, D. B. (1987). Multiple Imputation for Nonresponse in Surveys. New York: Wiley.

Sagi, E., Fu, Q. J., Galvin, J. J., 3rd, \& Svirsky, M. A. (2010). A model of incomplete adaptation to a severely shifted frequency-to-electrode mapping by cochlear implant users. J Assoc Res Otolaryngol, 11(1), 69-78.

Sagi, E., \& Svirsky, M. A. (2018). Deactivating cochlear implant electrodes to improve speech perception: A computational approach. Hear Res, 370, 316-328.

Saleh, S. M., Saeed, S. R., Meerton, L., Moore, D. R., \& Vickers, D. A. (2013). Clinical use of electrode differentiation to enhance programming of cochlear implants. Cochlear Implants Int, 14 Suppl 4, S16-18.

Santarelli, R., De Filippi, R., Genovese, E., \& Arslan, E. (2008). Cochlear implantation outcome in prelingually deafened young adults. A speech perception study. Audiol Neurootol, 13(4), 257-265.

Schramm, D., Fitzpatrick, E., \& Seguin, C. (2002). Cochlear implantation for adolescents and adults with prelinguistic deafness. Otol Neurotol, 23(5), 698-703.

Shannon, R. V. (1992). Temporal modulation transfer functions in patients with cochlear implants. J Acoust Soc Am, 91(4 Pt 1), 2156-2164.

Shannon, R. V., Cruz, R. J., \& Galvin, J. J., 3rd. (2011). Effect of stimulation rate on cochlear implant users' phoneme, word and sentence recognition in quiet and in noise. Audiol Neurootol, 16(2), 113-123.

Shannon, R. V., Zeng, F. G., Kamath, V., Wygonski, J., \& Ekelid, M. (1995). Speech recognition with primarily temporal cues. Science, 270(5234), 303-304.

Sharma, A., Campbell, J., \& Cardon, G. (2015). Developmental and cross-modal plasticity in deafness: evidence from the P1 and $\mathrm{N} 1$ event related potentials in cochlear implanted children. Int J Psychophysiol, 95(2), 135-144.

Sharma, A., Nash, A. A., \& Dorman, M. (2009). Cortical development, plasticity and re-organization in children with cochlear implants. J Commun Disord, 42(4), 272-279.

Shin, M. S., Song, J. J., Han, K. H., Lee, H. J., Do, R. M., Kim, B. J., \& Oh, S. H. (2015). The effect of psychosocial factors on outcomes of cochlear implantation. Acta Otolaryngol, 135(6), 572577.

Shpak, T., Koren, L., Tzach, N., Most, T., \& Luntz, M. (2009). Perception of speech by prelingual preadolescent and adolescent cochlear implant users. Int J Audiol, 48(11), 775-783. 


\section{References}

Skinner, M. W., Binzer, S. M., Fears, B. T., Holden, T. A., Jenison, V. W., \& Nettles, E. J. (1992). Study of the performance of four prelinguistically or perilinguistically deaf patients with a multielectrode, intracochlear implant. Laryngoscope, 102(7), 797-806.

Snik, A. F., Makhdoum, M. J., Vermeulen, A. M., Brokx, J. P., \& van den Broek, P. (1997). The relation between age at the time of cochlear implantation and long-term speech perception abilities in congenitally deaf subjects. Int J Pediatr Otorhinolaryngol, 41(2), 121-131.

Souza, I. P., Brito, R., Bento, R. F., Gomez, M. V., Tsuji, R. K., \& Hausen-Pinna, M. (2011). Speech perception in adolescents with pre-lingual hearing impairment with cochlear implants. Braz J Otorhinolaryngol, 77(2), 153-157.

Sparrow, R. (2010). Implants and ethnocide: learning from the cochlear implant controversy. Disability \& Society, 25(4), 455-466.

Straatman, L. V., Huinck, W. J., Langereis, M. C., Snik, A. F., \& Mulder, J. J. (2014). Cochlear implantation in late-implanted prelingually deafened adults: changes in quality of life. Otol Neurotol, 35(2), 253-259.

Teoh, S. W., Pisoni, D. B., \& Miyamoto, R. T. (2004a). Cochlear implantation in adults with prelingual deafness. Part I. Clinical results. Laryngoscope, 114(9), 1536-1540.

Teoh, S. W., Pisoni, D. B., \& Miyamoto, R. T. (2004b). Cochlear implantation in adults with prelingual deafness. Part II. Underlying constraints that affect audiological outcomes. Laryngoscope, 114(10), 1714-1719.

Tong, Y. C., Busby, P. A., \& Clark, G. M. (1988). Perceptual studies on cochlear implant patients with early onset of profound hearing impairment prior to normal development of auditory, speech, and language skills. J Acoust Soc Am, 84(3), 951-962.

Torrance, G. W., Feeny, D. H., Furlong, W. J., Barr, R. D., Zhang, Y., \& Wang, Q. (1996). Multiattribute utility function for a comprehensive health status classification system. Health Utilities Index Mark 2. Med Care, 34(7), 702-722.

van den Broek, P., Admiraal, R. J., Brokx, J., Mens, L., \& Spies, T. (1992). [Results of cochlear implants in totally deaf subjects]. Ned Tijdschr Geneeskd, 136(29), 1404-1409.

van der Marel, K. S., Briaire, J. J., Verbist, B. M., Muurling, T. J., \& Frijns, J. H. (2015). The influence of cochlear implant electrode position on performance. Audiol Neurootol, 20(3), 202-211.

van Dijkhuizen, J. N., Beers, M., Boermans, P. P., Briaire, J. J., \& Frijns, J. H. (2011). Speech intelligibility as a predictor of cochlear implant outcome in prelingually deafened adults. Ear Hear, 32(4), 445-458.

van Dijkhuizen, J. N., Boermans, P. P., Briaire, J. J., \& Frijns, J. H. (2016). Intelligibility of the Patient's Speech Predicts the Likelihood of Cochlear Implant Success in Prelingually Deaf Adults. Ear Hear, 37(5), e302-310.

van Wieringen, A., \& Wouters, J. (2008). LIST and LINT: sentences and numbers for quantifying speech understanding in severely impaired listeners for Flanders and the Netherlands. Int $J$ Audiol, 47(6), 348-355.

Vandali, A. E., Whitford, L. A., Plant, K. L., \& Clark, G. M. (2000). Speech perception as a function of electrical stimulation rate: using the Nucleus 24 cochlear implant system. Ear Hear, 21(6), 608-624.

Vickers, D., Degun, A., Canas, A., Stainsby, T., \& Vanpoucke, F. (2016). Deactivating Cochlear Implant Electrodes Based on Pitch Information for Users of the ACE Strategy. Adv Exp Med Biol, 894, 115-123.

Viemeister, N. F. (1979). Temporal modulation transfer functions based upon modulation thresholds. J Acoust Soc Am, 66(5), 1364-1380.

Waltzman, S. B., \& Cohen, N. L. (1999). Implantation of patients with prelingual long-term deafness. Ann Otol Rhinol Laryngol Suppl, 177, 84-87.

Waltzman, S. B., Cohen, N. L., \& Shapiro, W. H. (1992). Use of a multichannel cochlear implant in the congenitally and prelingually deaf population. Laryngoscope, 102(4), 395-399. 
Waltzman, S. B., Roland, J. T., Jr., \& Cohen, N. L. (2002). Delayed implantation in congenitally deaf children and adults. Otol Neurotol, 23(3), 333-340.

Ware, J. E., Jr., \& Sherbourne, C. D. (1992). The MOS 36-item short-form health survey (SF-36). I. Conceptual framework and item selection. Med Care, 30(6), 473-483.

Watson, S. D., Balko, K. A., Comer, L. K., Bishop, R. D., Reilley, D., \& Backous, D. D. (2003). Benefits of cochlear implantation in pre-lingual adult users: oral and manual communicators. Cochlear Implants Int, 4 Suppl 1, 75-76.

Won, J. H., Drennan, W. R., Nie, K., Jameyson, E. M., \& Rubinstein, J. T. (2011). Acoustic temporal modulation detection and speech perception in cochlear implant listeners. J Acoust Soc Am, 130(1), 376-388.

Won, J. H., Drennan, W. R., \& Rubinstein, J. T. (2007). Spectral-ripple resolution correlates with speech reception in noise in cochlear implant users. J Assoc Res Otolaryngol, 8(3), 384-392.

Won, J. H., Jones, G. L., Drennan, W. R., Jameyson, E. M., \& Rubinstein, J. T. (2011). Evidence of across-channel processing for spectral-ripple discrimination in cochlear implant listeners. $J$ Acoust Soc Am, 130(4), 2088-2097.

Xu, H. X., Kim, G. H., Snissarenko, E. P., Cureoglu, S., \& Paparella, M. M. (2012). Multi-channel cochlear implant histopathology: are fewer spiral ganglion cells really related to better clinical performance? Acta Otolaryngol, 132(5), 482-490.

Yang, W. S., Moon, I. S., Kim, H. N., Lee, W. S., Lee, S. E., \& Choi, J. Y. (2011). Delayed cochlear implantation in adults with prelingual severe-to-profound hearing loss. Otol Neurotol, 32(2), 223-228.

Yucel, E., \& Sennaroglu, G. (2007). Is psychological status a determinant of speech perception outcomes in highly selected good adolescent cochlear implant users? Int J Pediatr Otorhinolaryngol, 71(9), 1415-1422.

Zeitler, D. M., Anwar, A., Green, J. E., Babb, J. S., Friedmann, D. R., Roland, J. T., Jr., \& Waltzman, S. B. (2012). Cochlear implantation in prelingually deafened adolescents. Arch Pediatr Adolesc Med, 166(1), 35-41.

Zwolan, T. A., Collins, L. M., \& Wakefield, G. H. (1997). Electrode discrimination and speech recognition in postlingually deafened adult cochlear implant subjects. J Acoust Soc Am, 102(6), 3673-3685.

Zwolan, T. A., Kileny, P. R., \& Telian, S. A. (1996). Self-report of cochlear implant use and satisfaction by prelingually deafened adults. Ear Hear, 17(3), 198-210. 

Dankwoord 

Eindelijk is het zover... mijn boekje is af! Ik wil graag iedereen bedanken die mij op de weg hiernaartoe heeft geholpen en gesteund.

Dr. Brokx, beste Jan, mijn eerste woord van dank gaat uit naar jou. Ik herinner me nog heel goed hoe je, niet zo heel lang nadat ik in Maastricht aan de slag was gegaan, voorstelde om samen "een projectje" uit te werken. Dat dit wel eens tot een proefschrift zou kunnen leiden, daar stond ik toen helemaal nog niet bij stil. En ziehier... Ik prijs mezelf heel erg gelukkig jou als directe begeleider te hebben gehad. Met je verhalen en filmpjes uit "de oude doos" bracht je de wetenschap tot leven! Je enthousiasme werkte heel aanstekelijk, maar nog belangrijker voor mij was je kunst om te relativeren én het grotere plaatje voor ogen te houden. Boven alles heb ik het enorm gewaardeerd dat je mij als persoon altijd op de eerste plaats zette. Jan, bedankt voor alles!

Prof. Kremer, beste Bernd, ik wil je van harte danken voor je steun tijdens mijn promotietraject. Je gaf me op het juiste moment telkens die duwtjes in de rug die nodig waren om een nieuwe stap voorwaarts te zetten. Onze bijeenkomsten creëerden structuur en zorgden ervoor dat ik mijn doel steeds voor ogen hield. Jouw vertrouwen in mij heeft me heel erg geholpen om door te zetten en dit proefschrift af te werken. Bedankt daarvoor!

Prof. Francart, beste Tom, jouw hulp bij het totstandkomen van de experimentele opstellingen uit hoofdstukken 2 en 3 was onmisbaar! Door jouw adviezen en tips, waarbij je telkens weer een tipje van de sluier oplichtte, heb ik heel veel bijgeleerd. Maar ook daarna bij het interpreteren van de bevindingen was het heel waardevol jou als sparring partner te hebben. Dankjewel voor alles!

Prof. Stokroos, beste Robert, bedankt voor je enthousiasme en adviezen tijdens de eerste jaren van mijn promotie!

Aan de leden van de beoordelingscommissie, prof. dr. W. Mess, prof. dr. R. van Oostenbrugge, prof. dr. E. Gerrits, dr. E. Mylanus en prof. dr. H. Kingma, hartelijk dank voor uw bereidheid om mijn proefschrift te beoordelen.

Miranda en Mickey, wat is het een zegen om als promovendus op jullie statistische knowhow te mogen rekenen! Geen vraag was ooit teveel, en altijd was er wel een gaatje in de agenda te vinden om even een probleem voor te leggen of een SPSS-uitdraai tot leven te laten komen. Gewoon "goed" was zeker niet goed genoeg, jullie gingen steeds tot het uiterste. Bedankt! 
Anke, ik zie ons nog zitten aan je keukentafel, samen letterlijk tussen de pampers ploeterend om de comments van de reviewers van een goed antwoord te voorzien. Wat een hectische maar ook leuke tijd was dat! Het was een fantastische ervaring om samen met jou die eerste publicatie erdoor te krijgen. Heel erg bedankt voor je enthousiasme, doorzettingsvermogen, maar vooral je vrienschap!

Katrien, precies op het moment dat ik je nodig had kwam jij op het AC aanwaaien. We hebben samen super goed samengewerkt, en vooral heel veel plezier gemaakt. Dankzij jouw vrolijkheid voelde het vaak zelfs niet eens aan als werken! Ik bewonder je enorme inzet, en hoe jij steeds voor de volle $100 \%$ voor iets gaat. Een dikke merci voor alle babbels, waarvan er hopelijk nog veel volgen!

Kim, jouw hulp en technische ondersteuning bij het totstandkomen van hoofdstuk 3 heb ik enorm gewaardeerd. Super bedankt voor de gezellige en leerrijke samenwerking!

Aan al mijn lieve collega's... wat een voorrecht om samen met jullie ons AC draaiende te mogen houden en zoveel patiënten te kunnen helpen. Nietsvermoedend kwam ik 13 jaar geleden in Maastricht terecht voor wat een onvergetelijke stage zou gaan worden, en een dik half jaar later ook mijn eerste en meteen fantastische werkplek. Afra, Angélique, Anne, Annemie, Ans, Audrey H., Audrey V., Danielle, Denise, Edith, Els, Erwin, Eveline, Gijs, Gina, Iris, Jogina, Judith, Lucien, Mirçea, Nadia, Peter, Raymond, Sander, Sandra, Skadi, Sophie, Stan, Winde, Xandra en Yvonne: bedankt voor jullie vele, steeds luisterende oren...

In het bijzonder Els, Nadia en Winde: bedankt om zulke lieve, attente en begripvolle collega's en vriendinnnen te zijn... jullie zijn goud waard! Xandra en Angélique, jullie zijn naast fantastische collega's een enorme hulp geweest bij het verzamelen van al mijn data, waarbij nooit iets teveel gevraagd was. Bedankt daarvoor! Mirçea, dank voor alle PMPuitdraaien "op bestelling". Anne, aan het uitwisselen van onze promotie-ervaringen heb ik veel deugd gehad. Nu ook jij nog heel even volhouden! Ester, jouw praktische hulp bij de laatste loodjes was een hele steun en geruststelling!

Lucien, ik ga nooit vergeten (en dat heb ik je niet altijd in dank afgenomen (:)!) hoe het jouw idee was om een systematic review te schrijven, maar ook dat je mij in afwezigheid van Jan zo goed mogelijk hebt proberen te begeleiden. Lieve Jogina, toen je heel dapper terug aan het werk probeerde te gaan heb je nog een groot deel van mijn verzamelde data in PMP ingevoerd, waarvoor ik je heel dankbaar ben. Er zullen voor altijd twee stukjes uit onze AC-puzzel ontbreken.

Karen, ik kijk met heel veel plezier terug op onze $\mathrm{Cl}$-trainingen samen, waarbij we onze kennis telkens op een heel gezellige manier verder uitbreidden! Later kwam ook Sander het $\mathrm{Cl}$-clubje in Venlo vervoegen. Super bedankt allebei, en bij uitbreiding iedereen van het team in Venlo, voor de fijne samenwerking! 
Aan het "Elephant-team": Elke, Lars, Marc, Erwin en Kiki. Ik ben heel blij deel uit te kunnen maken van deze enthousiaste onderzoeksgroep, en kijk uit naar onze verdere samenwerking. Elke, bedankt voor alle gezellige promotie- en andere babbels. Jij mag nu ten volle genieten! Lars... heel veel succes! $: ;$

Aan alle "proefpersonen" uit dit proefschrift: heel erg bedankt voor jullie inzet tijdens de soms bijna eindeloze reeks testen. Zonder jullie geen data, geen proefschrift, en bovenal geen nieuwe kennis. Bedankt!

Kirsten, heel erg bedankt voor alle creativiteit, inzet en tijd die je in het ontwerpen van de cover van dit boekje gestoken hebt!

Aan mijn (schoon-)familie en vrienden, die steeds weer met interesse informeerden naar de stand van zaken van mijn "onderzoek": jullie geduld wordt eindelijk beloond!

Speciale dank aan de "babysit-oma's", mama en moeke, voor alle (extra) babysit-uurtjes op Nuria en Gioia én de ontelbare manden strijk, die mij telkens weer toelieten een stapje verder richting het afwerken van dit proefschrift te komen. Moeke, dankjewel om er steeds te zijn voor ons gezinnetje.

Lieve mama en papa, David en Isabelle, wat een luxe en geluk dat ik ben mogen opgroeien in zo'n warm gezin, en hier ook nog steeds op kan terugvallen. Mama en papa, bedankt voor alle alle mogelijkheden die jullie mij gegeven hebben, en om altijd voor mij klaar te staan!

Liefste Bert, ook voor $\mathrm{u}$ is het einde van een hele intense periode bijna in zicht, waardoor we binnenkort allebei een bladzijde in ons leven omslaan. Ik ben benieuwd naar wat de toekomst nog voor ons in petto heeft en kijk er naar uit die samen te ontdekken! Nuria en Gioia, mijn lieve, mooie meisjes... rustig aan mijn proefschrift werken was met jullie in huis meestal geen optie, maar wat ben ik blij dat jullie in mijn leven gekomen zijn. Jullie verder te zien opgroeien is het mooiste cadeau dat ik me kan wensen! 



\section{Curriculum Vitae}

Joke Debruyne werd geboren op 21 juli 1984 te Asse, België en groeide op in Sint-Amands, aan de Schelde. Na het doorlopen van haar middelbare school aan de Onze-Lieve-Vrouw Presentatie te Bornem, waar zij de richting Moderne Talen - Wiskunde volgde, startte zij in 2002 met de opleiding Logopedische \& Audiologische Wetenschappen aan de Katholieke Universiteit Leuven (KULeuven, België). Zij koos voor de afstudeerrichting audiologie en behaalde in $\mathbf{2 0 0 6}$ haar masterdiploma met grote onderscheiding. Voor haar masterthesis, die handelde over auditieve discriminatie bij kinderen met ontwikkelingsstotteren, ontving zij van de docenten de Outstanding Master Thesis Award. In 2007 vervolledigde zij haar opleiding met een diploma van de academische lerarenopleidng en een postgraduaat hoortoestelaanpassing. Tijdens het laatste jaar van haar opleiding liep zij gedurende 3 maanden stage bij het audiologisch centrum van het Maastricht UMC+ en ging hier kort na haar afstuderen aan de slag. Als master audioloog staat zij in voor de audiologische zorg binnen het volwassenteam. Naast haar klinische werkzaamheden startte zij met een promotietraject onder begeleiding van dr. ir. Jan Brokx en prof. dr. Bernd Kremer (Maastricht UMC+), en met de externe steun van prof. dr. ir. Tom Francart (KULeuven), hetwelke resulteerde in dit proefschrift. 



\section{List of publications}

1. De Ruiter, A.M., Debruyne, J., Chenault, M.N., Francart, T., Brokx, J.P.L. (2015). Amplitude Modulation Detection and Speech Recognition in Late-Implanted Prelingually and Postlingually Deafened Cochlear Implant Users. Ear Hear, 36(5), 55766.

2. Debruyne, J., Francart, T., Janssen, A.M., Douma, K., Brokx, J.P. (2016). Fitting prelingually deafened adult cochlear implant users based on electrode discrimination performance. Int J Audiol, 56(3), 174-185.

3. Debruyne, J., Janssen, M., Brokx, J. (2017). Late Cochlear Implantation in EarlyDeafened Adults: A Detailed Analysis of Auditory and Self-Perceived Benefits. AudiolNeurootol, 22(3), 364-376.

4. Vaerenberg B, Smits C, De Ceulaer G, Zir E, Harman S, Jaspers N, Tam Y, Dillon M, Wesarg T, Martin-Bonniot D, Gärtner L, Cozma S, Kosaner J, Prentiss S, Sasidharan P, Briaire JJ, Bradley J, Debruyne J, Hollow R, Patadia R, Mens L, Veekmans K, Greisiger R, Harboun-Cohen E, Borel S, Tavora-Vieira D, Mancini $P$, Cullington $H, N g ~ A H$, Walkowiak A, Shapiro WH, Govaerts PJ. (2014). Cochlear implant programming: a global survey on the state of the art. ScientificWorldJournal, 2014:501738 